\title{
Directed Wavelet Covariance for Locally Stationary Processes
}

\author{
Kim Samejima Mascarenhas Lopes \\ TESE APRESENTADA \\ AO \\ Instituto de MATEMÁticA E EstatísticA \\ DA \\ UNIVERSIDADE DE SÃo PAULO \\ PARA \\ OBTENÇÃO DO TÍTULO \\ $\mathrm{DE}$ \\ DOUTOR EM CIÊNCIAS
}

Programa: Estatística

Orientador: Prof. Dr. Pedro Alberto Morettin

Coorientador: Prof. Dr. João Ricardo Sato

São Paulo, 12 de Março de 2018 


\section{Directed Wavelet Covariance for Locally Stationary Processes}

Esta versão da tese contém as correções e alterações sugeridas pela Comissão Julgadora durante a defesa da versão original do trabalho, realizada em 12/03/2018. Uma cópia da versão original está disponível no Instituto de Matemática e Estatística da Universidade de São Paulo.

Comissão Julgadora:

- Prof. Dr. Pedro Alberto Morettin - IME-USP

- Profa. Dra. Chang Chiann - IME-USP

- Prof. Dr. Aluísio de Souza Pinheiro - IMECC-UNICAMP

- Dr. Rogério de Faria Porto - Banco do Brasil

- Prof. Dr. Ronaldo Dias - IMECC-UNICAMP 
Dedicated to my grandmother

おばあちやん、あなたの扔かげで わたしわなんでもできるように なりました! ありがとうございました! 


\section{Acknowledgements}

I would like to thank my supervisors, Pedro and João, for their outstanding support, patience, and kindness. I am very comfortable to say that I could achieve nothing in my academic life without their guidance and friendship.

I also want to thank the Institute of Mathematics and Statistics of UFBA for allowing me full-time dedication to this program and my family, especially my mother, Erika and my brothers, Caio and Keny, for all the love and comprehension during my uncountable study nights.

I am grateful to Lucas Trambaiolli for providing the EEG data presented on Chapter 6 .

Also, I am very thankful to the HPC-USP team, especially my friend Ettore, who supported and helped me a lot with all the processing on HPC servers Aguia and Lince.

Finally, I would like to thank my friends Ednei Reis, Fabio Kacuta, Felipe Trevizan, Heitor Marcos, Lia Terezinha, Paulo Lima, Pedro Hinckel and Talita Cordeiro who, each one on his/her unique way, contributed to making this thesis possible, my life happier and my decisions wiser. 


\section{Resumo}

Samejima, K. Covariância Direcionada de Ondaletas para Processos Localmente Estacionários.

2018. 128 p. Tese (Doutorado) - Instituto de Matemática e Estatística, Universidade de São Paulo, São Paulo, 2018.

O objetivo deste trabalho é propor uma metodologia para mensurar o impacto direcionado entre processos localmente estacionários. Diferente de processos estacionários, processos localmente estacionários podem apresentar mudanças bruscas e características específicas em determinados intervalos. Tal comportamento pode causar instabilidade em medidas baseadas na transformada de Fourier. A importância deste estudo se dá em englobar processos com tais características, propondo metodologias robustas que não são afetadas pela existência de mudanças bruscas, pontos discrepantes e comportamentos locais.

Inicialmente apresentamos conceitos já existentes na literatura, como a Coerência Parcial Direcionada (PDC) e a Coerência de Ondaletas. A PDC mede o impacto direcionado entre componentes de um modelo vetorial autoregressivo (VAR) no domínio da frequência. A coerência de ondaletas é baseada em transformadas complexas de ondaletas. Propomos então uma decomposição no domínio de ondaletas para a estrutura de covariância de processos bivariados localmente estacionários: a Covariância Direcionada de Ondaletas (DWC). Em comparação com as quantidades baseadas na tranformada Fourier, os estimadores baseados em ondaletas são mais apropriados para processos não estacionários com padrões locais, pontos discrepantes ou mudanças rápidas de regime, como em experimentos de eletroencefalograma (EEG) com a introdução de estímulo. Ainda, propomos um estimador para a DWC, calculamos a esperança deste estimador e avaliamos sua variância.

Em seguida, propomos uma quantidade análoga à DWC para processos multivariados com mais de duas componentes: a Covariância Parcial Direcionada de Ondaletas (pDWC). Considerando um processo $N$-variado localmente estacionário, a pDWC calcula a Covariância Direcionada de Ondaletas entre $X_{1}(t)$ e $X_{2}(t)$ eliminando o efeito das outras componentes $X_{3}(t), \ldots, X_{N}(t)$. Propomos duas abordagens para a pDWC: na primeira, a pDWC é calculada após a aplicação de um filtro linear que remove o efeito das variáveis exógenas. No segundo caso, a exemplo da Coerência Parcial Direcionada, consideramos o processo multivariado como um Modelo Autoregressivo de Vetorial variante no tempo (tv-VAR) e usamos seus coeficientes na decomposição da função de covariância para isolar os efeitos das demais componentes. 
Também comparamos os resultados da PDC, Coerência de Ondaletas e Covariância Direcionada de Ondaletas com dados simulados. Por fim, apresentamos uma aplicação da DWC e da pDWC em dados de EEG. Identificamos nas simulações que tanto as medidas já existentes na literatura quanto as quantidades propostas identificaram as relações simuladas. A pDWC proposta com filtros lineares apresentou estimações mais estáveis do que a pDWC considerando os modelos tv-VAR. Estudos futuros discutirão as propriedades assintóticas e testes de significância da DWC e pDWC.

Palavras-chave: Séries Temporais, Processos Localmente Estacionários, Espectro Cruzado, Coerência Direcionada, Covariância de Ondaletas. 


\section{Abstract}

Samejima, K. Directed Wavelet Covariance for Locally Stationary Processes. 2018. 128 p. Tese (Doutorado) - Instituto de Matemática e Estatística, Universidade de São Paulo, São Paulo, 2016.

The main goal of this study is to propose a methodology that measures directed relations between locally stationary processes. Unlike stationary processes, locally stationary processes may present sudden pattern changes and have local characteristics in specific intervals. This behavior causes instability in measures based on Fourier transforms. The relevance of this study relies on considering these processes and propose robust methodologies that are not affected by outliers, sudden pattern changes or local behavior.

We start reviewing the Partial Directed Coherence (PDC) and the Wavelet Coherence. PDC measures the directed relation between components of a multivariate stationary Vector Autoregressive (VAR) model in the frequency domain, while Wavelet Coherence is based on complex wavelets decomposition. We then propose a causal wavelet decomposition of the covariance structure for bivariate locally stationary processes: the Directed Wavelet Covariance (DWC). Compared to Fourierbased quantities, wavelet-based estimators are more appropriate for non-stationary processes and processes with local patterns, outliers and rapid regime changes like in EEG experiments with the introduction of stimuli. We then propose its estimators and calculate its expectation and analyze its variance.

Next we propose a decomposition for the variance of multivariate processes with more than two components: the Partial Directed Wavelet Covariance (pDWC). Considering a N-variate locally stationary process, the pDWC calculates the Directed Wavelet Covariance of $X_{1}(t)$ with $X_{2}(t)$ eliminating the effect of the other components $X_{3}(t), \ldots, X_{N}(t)$. We propose two approaches to this situation. First we filter the multivariate process to remove all the exogenous influences and then we calculate the directed relation between the components. In the second case, as in Partial Directed Coherence, we consider the multivariate process as a time-varying Vector Autoregressive Model (tvVAR) and use its coefficients in the decomposition of the covariance function to isolate the effects of the other components.

We also compare results of the PDC, Wavelet Coherence and Directed Wavelet Covariance with simulated data. Finally, we present an application of the proposed Directed Wavelet Covariance and Partial Directed Wavelet Covariance on EEG data. Simulation results show that the proposed 
measures capture the simulated relations. The pDWC with linear filter has shown more stable estimations than the proposed pDWC considering the tv-VAR. Future studies will discuss the DWC's and pDWC's asymptotic distributions and significance tests.

The proposed Directed Wavelet Covariance decomposition is a different approach to deal with non-stationary processes in the context of causality. The use of wavelets is a gain and adds to the number of studies that can be addressed when Fourier transform does not apply. The pDWC is an alternative for multivariate processes and it removes linear influences from observed external components.

Keywords: Time series, Locally Stationary Processes, Cross Spectrum, Directed Coherence, Wavelet Covariance. 


\section{Contents}

List of Abbreviations $\quad$ xi

List of Symbols $\quad$ xiii

$\begin{array}{ll}\text { List of Figures } & \text { xv }\end{array}$

List of Tables $\quad$ xix

1 Introduction 1

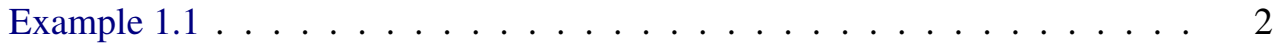

2 Methods 5

2.1 Spectral Analysis . . . . . . . . . . . . . . . . . . . . . 5

2.1.1 Fourier Transform of Processes with Discrete Time . . . . . . . . . . . . 5

2.1.2 Spectral Density Function and Cross Spectrum . . . . . . . . . . . . . 6

Example $2.1 \ldots \ldots \ldots \ldots \ldots$. . . . . . . . . . . . . 7

2.1.3 Coherence and Partial Coherence _................ . . 9

2.2 Partial Directed Coherence ．.. . . . . . . . . . . . . . . . . . . . . . 11

Example $2.2 \ldots \ldots \ldots \ldots \ldots \ldots$

Example $2.3 \ldots \ldots \ldots \ldots \ldots$. . . . . . . . . . . . . . . . . . 14

2.3 Locally Stationary Processes and Evolutionary Spectrum . . . . . . . . . . . . 15

2.4 Wavelets . . . . . . . . . . . . . . . . . . . . . . . . . . . . . . 19

2.4 .1 Multiresolution Analysis . . . . . . . . . . . . . . . . 22

2.4.2 Continuous Wavelet Transform _. . . . . . . . . . . . . . 23

2.4 .3 Discrete Wavelet Transform . . . . . . . . . . . . . . . . . 23

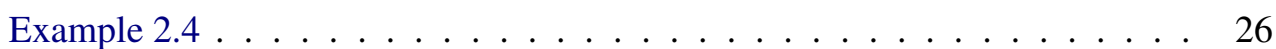

Example $2.5 \ldots \ldots \ldots \ldots \ldots \ldots \ldots$

2.4.4 Maximal Overlap Discrete Wavelet Transform . . . . . . . . . . . . . . 28

Example $2.6 \ldots \ldots \ldots \ldots \ldots \ldots$

2.5 Wavelet Covariance . . . . . . . . . . . . . . . . . . . . 32

2.6 Wavelet Coherence . . . . . . . . . . . . . . . . . . . . . . . 33

3 Directed Wavelet Covariance 35

3.1 Estimation . . . . . . . . . . . . . . . . . . . . . . 36 
3.1.1 Properties of the Estimators: Expectation and Variance . . . . . . . . . . . 37

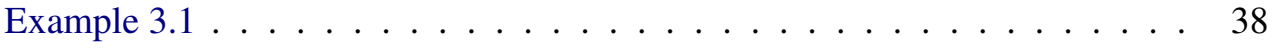

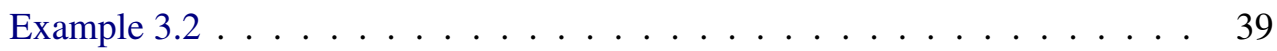

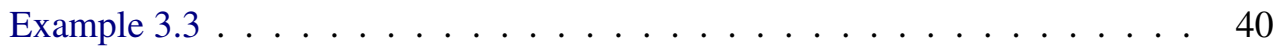

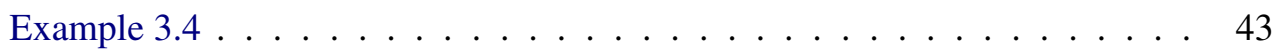

4 Partial Directed Wavelet Covariance $\quad 47$

4.1 pDWC with a linear filter: pDWC . . . . . . . . . . . . . . . . . . . 47

4.2 pDWC assuming a tv-VAR(p) model: ppDWC . . . . . . . . . . . . . . . . . . 48

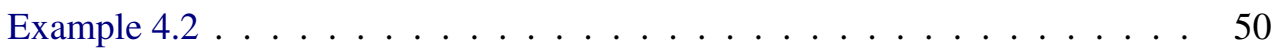

Simulation $4.3 \ldots \ldots \ldots 3$

Simulation $4.4 \ldots \ldots \ldots 54$

Simulation $4.5 \ldots \ldots \ldots 54$

5 Simulations $\quad 59$

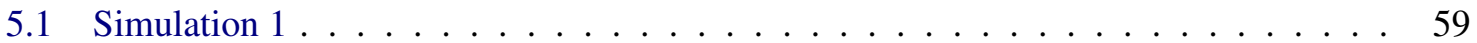

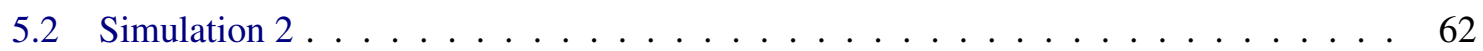

5.3 Simulation 3 ......................... 67

5.4 Remarks ............................. 67

6 An application to EEG Data $\quad 69$

6.1 DWC on EEG data . . . . . . . . . . . . . . . . . . . . 70

6.2 pDWC on EEG data . . . . . . . . . . . . . . . . . . . . . . 74

6.3 Remarks ............................ 75

7 Discussion $\quad 79$

$\begin{array}{lr}\text { A Figures } & \mathbf{8 5}\end{array}$

B Proofs $r 11$

Bibliography $r$

$\begin{array}{lr}\text { Index } & 128\end{array}$ 


\title{
List of Abbreviations
}

\author{
CFT Continuous Fourier Transform \\ DFT Discrete Fourier Transform \\ FFT Fast Fourier Transform \\ CWT Continuous Wavelet Transform \\ DWT Discrete Wavelet Transform \\ MODWT Maximal Overlap Discrete Wavelet Transform \\ DC Directed Coherence \\ PDC Partial Directed Coherence \\ XWT Cross Wavelet Transform \\ WTC Wavelet Coherence \\ WN White Noise process \\ DWC Directed Wavelet Covariance \\ pDWC Partial Directed Wavelet Covariance using linear filters \\ ppDWC Partial Directed Wavelet Covariance assuming tv-VAR(p) structure \\ VAR Vector Autoregressive model \\ VMA Vector Moving Average model \\ tv-VAR time-varying Vector Autoregressive model
}




\section{List of Symbols}

\begin{tabular}{|c|c|}
\hline$i$ & imaginary unit, i.e. $i^{2}=-1$ \\
\hline$\omega$ & Frequency \\
\hline$X_{t, T}$ & A real valued time series with discrete times $t \in 0, \ldots, T$ \\
\hline$\pi_{i, j}(\omega)$ & Partial Directed Coherence between components $X_{i}(t)$ and $X_{j}(t)$. \\
\hline$\psi(t)$ & Father Wavelet $\psi$ \\
\hline$\phi(t)$ & Mother Wavelet $\psi$ \\
\hline$\left\{h_{k}\right\}$ & DWT filter \\
\hline$\left\{l_{k}\right\}$ & DWT Scaling filter \\
\hline$\left\{\widetilde{h}_{k}\right\}$ & MODWT filter \\
\hline$\left\{\widetilde{l}_{k}\right\}$ & MODWT Scaling filter \\
\hline$W_{j, t}$ & Discrete Wavelet Transform in scale $\tau_{j}$ and time $t$ \\
\hline$\widetilde{W}_{j, t}$ & Maximal Overlapped Discrete Wavelet Transform at level $\mathrm{j}$ and time $t$ \\
\hline$\nu_{X, t}^{2}\left(\tau_{j}\right)$ & Time-dependent wavelet variance of $X_{t}$ at scale $\tau_{j}$ \\
\hline$\nu_{X Y, t}\left(\tau_{j}\right)$ & Wavelet covariance between $X_{t}$ and $Y_{t}$ at scale $\tau_{j}$ \\
\hline$\gamma_{X Y}\left(t_{1}, t_{2}\right)$ & Covariance function between $X_{t_{1}}$ and $Y_{t_{2}}$ \\
\hline$F_{X Y, t}\left(\tau_{j}\right)$ & Directed Wavelet Covariance from $X_{t}$ to $Y_{t}$ at scale $\tau_{j}$ \\
\hline$B_{X Y, t}\left(\tau_{j}\right)$ & Directed Wavelet Covariance from $Y_{t}$ to $X_{t}$ at scale $\tau_{j}$ \\
\hline$p F_{X Y, t}\left(\tau_{j}\right)$ & Partial Directed Wavelet Covariance from $X_{t}$ to $Y_{t}$ at scale $\tau_{j}$ using linear filters \\
\hline$p B_{X Y, t}\left(\tau_{j}\right)$ & Partial Directed Wavelet Covariance from $Y_{t}$ to $X_{t}$ at scale $\tau_{j}$ using linear filters \\
\hline$p p F_{X Y, t}\left(\tau_{j}\right)$ & Partial Directed Wavelet Covariance from $X_{t}$ to $Y_{t}$ at scale $\tau_{j}$ assuming tv-VAR(p) \\
\hline$p p B_{X Y, t}\left(\tau_{j}\right)$ & Partial Directed Wavelet Covariance from $Y_{t}$ to $X_{t}$ at scale $\tau_{j}$ assuming tv-VAR(p) \\
\hline$\hat{c_{T}}(u, k)$ & Estimator of the Covariance function between $X_{u}$ and $Y_{u+k}$ \\
\hline$\widehat{F}_{X Y, t}\left(\tau_{j}\right)$ & Estimator of the Directed Wavelet Covariance from $X_{t}$ to $Y_{t}$ at scale $\tau_{j}$ \\
\hline$\widehat{B}_{X Y, t}\left(\tau_{j}\right)$ & Estimator of the Directed Wavelet Covariance from $Y_{t}$ to $X_{t}$ at scale $\tau_{j}$ \\
\hline $\mathbb{E}()$. & Expectation Function \\
\hline $\operatorname{Cov}()$. & Covariance Function \\
\hline $\operatorname{Var}()$. & Variance Function \\
\hline$J$ & largest DWT level for sample size $N=2^{J}$ \\
\hline$L$ & Scaling or Wavelet filter width \\
\hline$L_{j}$ & Wavelet filter width of $j$-th level \\
\hline$L^{2}(\mathbb{R})$ & Set of square integrable real-valued function \\
\hline$n \bmod N$ & $n$ modulo $N$ \\
\hline $\mathbb{R}$ & The entire Real axis \\
\hline $\mathbb{C}$ & The set of Complex numbers \\
\hline $\mathbb{Z}$ & The set of Integer numbers \\
\hline$f_{X}(\omega)$ & Spectral Density Function of $X$ at $\omega$ frequency \\
\hline$K()$. & Kernel Function \\
\hline
\end{tabular}




\section{List of Figures}

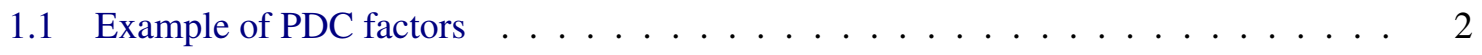

1.2 Examples of Fourier Approximation $\ldots \ldots \ldots \ldots \ldots$

2.1 Examples of Spectral Density Functions . . . . . . . . . . . . . . . . . . 8

2.2 Example of simulated $\operatorname{VAR}(1) \ldots \ldots \ldots \ldots \ldots \ldots \ldots \ldots$

2.3 PDC for the simulated VAR(1) in Example $2.2 \ldots \ldots \ldots \ldots$

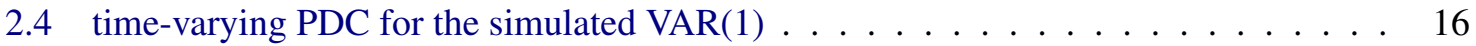

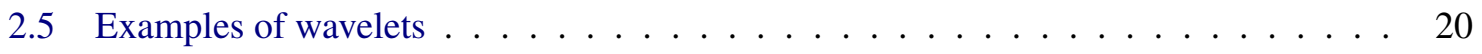

2.6 Illustration of orthonormal complement $W_{0} \ldots \ldots \ldots \ldots \ldots \ldots \ldots$

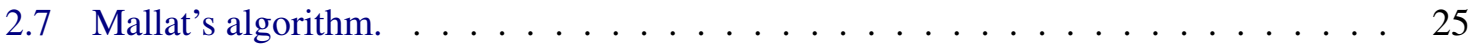

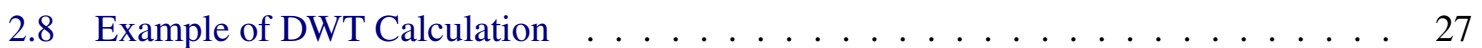

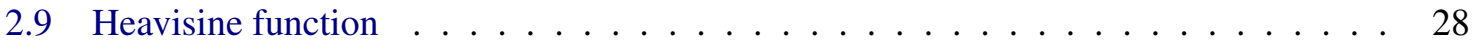

2.10 Example of DWT Calculation for heavisine function $\ldots \ldots \ldots \ldots$

2.11 Time series for Example $2.6 \ldots \ldots \ldots$

2.12 Impact of shifting the time series in DWT coefficients . . . . . . . . . . . . . 30

2.13 Impact of shifting the time series in MODWT coefficients . . . . . . . . . . . . . 31

2.14 Example of MODWT Calculation. . . . . . . . . . . . . . . . . 31

2.15 Example of MODWT Calculation for heavisine function $\ldots \ldots \ldots$. . . . . . . 32

2.16 Wavelet Coherence for Example $2.3 \ldots \ldots$. . . . . . . . . . . . 34

3.1 Example 3.2. Theoretical values for $F_{12, t}\left(\tau_{j}\right)$ and $B_{12, t}\left(\tau_{j}\right)$ for each level. . . . . . 41

3.2 Average $F_{12, t}\left(\tau_{j}\right)$ and $B_{12, t}\left(\tau_{j}\right)$ in Example $3.2 \ldots \ldots \ldots \ldots \ldots \ldots \ldots$

3.3 Example 3.3. Theoretical values for $F_{12, t}\left(\tau_{j}\right)$ and $B_{12, t}\left(\tau_{j}\right)$ for each level. . . . . . . 44

3.4 Average $F_{12, t}\left(\tau_{j}\right)$ and $B_{12, t}\left(\tau_{j}\right)$ in Example 3.3 with $a=b=0.6 \ldots \ldots \ldots$. . . 44

3.5 Average $F_{12, t}\left(\tau_{j}\right)$ and $B_{12, t}\left(\tau_{j}\right)$ in Example 3.3 with $a=0.6$ and $b=0.3 \ldots \ldots$. . . 44

3.6 Example 3.4. Theoretical values for $F_{12, t}\left(\tau_{j}\right)$ and $B_{12, t}\left(\tau_{j}\right)$ for each level. . . . . . 46

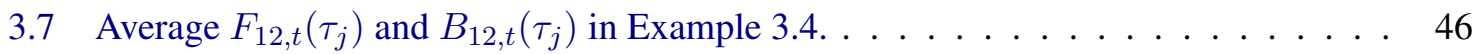

4.1 Relations between components of the process $(4.4) \ldots \ldots \ldots \ldots$

4.2 Relations between components $X_{1, t}$ and $X_{2, t}$ in process (4.4) estimated by pDWC. . 51

4.3 Relations between components $X_{1, t}$ and $X_{3, t}$ in process (4.4) estimated by pDWC. . 51

4.4 Relations between components $X_{2, t}$ and $X_{3, t}$ in process (4.4) estimated by pDWC. . 51

4.5 Average Partial Directed Wavelet Covariance (ppDWC) for Example 4.3 . . . . . . . 54

4.6 Average Partial Directed Wavelet Covariance (ppDWC) for Example 4.4 . . . . . . . 55 
4.7 Average Partial Directed Wavelet Covariance (ppDWC) for Example 4.5 . . . . . . . 56

4.8 Example 4.6: Relations between components $X_{1, t}$ and $X_{2, t}$ in process (4.4) estimated

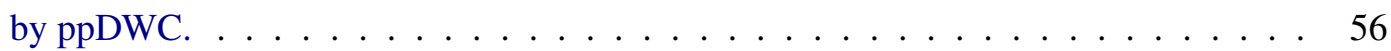

4.9 Example 4.6: Relations between components $X_{1, t}$ and $X_{3, t}$ in process (4.4) estimated

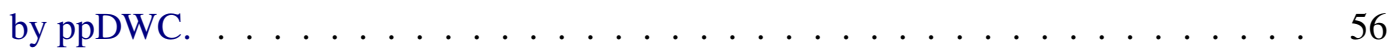

4.10 Example 4.6: Relations between components $X_{2, t}$ and $X_{3, t}$ in process (4.4) estimated

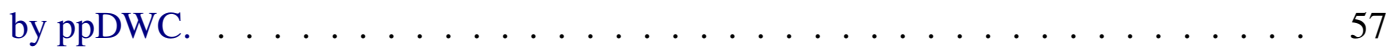

5.1 Example of Simulation $1 \ldots \ldots \ldots \ldots$

5.2 WTC for Simulation $1 \ldots \ldots \ldots \ldots$

5.3 PDC for Simulation $1 \ldots \ldots \ldots \ldots$. . . . . . . . . . . . . . 61

5.4 DWC for Simulation 1 - one time series $\ldots \ldots \ldots$. . . . . . . . . . 62

5.5 Average DWC for Simulation $1 \ldots \ldots \ldots$. . . . . . . . . . . . . . 62

5.6 Bootstrap for DWC in for Simulation 1 . . . . . . . . . . . . . . . 63

5.7 Example of Simulation $2 \ldots \ldots \ldots \ldots$. . . . . . . . . . . . . . 64

5.8 WTC for Simulation $2 \ldots \ldots \ldots \ldots$

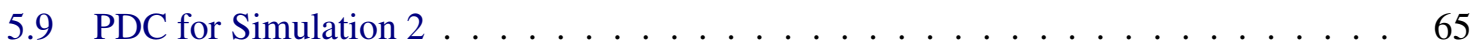

5.10 DWC for Simulation 2 - one time series . . . . . . . . . . . . . . . . 66

5.11 Average DWC for Simulation 2 . . . . . . . . . . . . . . . . . 66

5.12 Coefficients used in Simulation $2 \ldots \ldots \ldots$. . . . . . . . . . 67

5.13 Example of Simulation $3 \ldots \ldots \ldots$. . . . . . . . . . . . . . 68

6.1 Subsampled EEG channels C3, Cz and C4 . . . . . . . . . . . . . . . . 69

6.2 Illustration of the EEG experiment . . . . . . . . . . . . . . . . 70

6.3 Position of EEG channels . . . . . . . . . . . . . . . . . . . . . . . 71

6.4 DWC for the EEG data . . . . . . . . . . . . . . . . . . . 72

6.5 Comparison between types of trials . . . . . . . . . . . . . . . . . . 74

7.1 Average DWC for 100 replications of model (5.1) estimated using Haar wavelets,

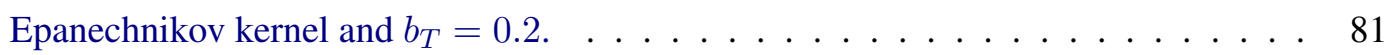

7.2 Average DWC for 100 replications of model (5.1) estimated using Haar wavelets, Epanechnikov kernel and $b_{T}=0.7 \ldots \ldots \ldots \ldots$. . . . . . . . . 81

7.3 Average DWC for 100 replications of model (5.1) estimated using Haar wavelets,

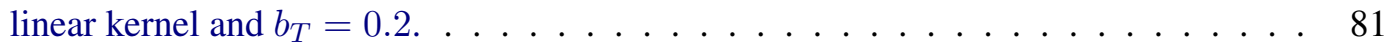

A.1 Empirical Distribution for $F_{12, t}\left(\tau_{j}\right)$ and $B_{12, t}\left(\tau_{j}\right)$ in Example 3.2. $\ldots \ldots \ldots \ldots$

A.2 Empirical Distribution for $F_{12, t}\left(\tau_{j}\right)$ and $B_{12, t}\left(\tau_{j}\right)$ in Example 3.3 with $a=b=0.6$. . 86

A.3 Empirical Distribution for $F_{12, t}\left(\tau_{j}\right)$ and $B_{12, t}\left(\tau_{j}\right)$ in Example 3.3 with $a=0.6$ and

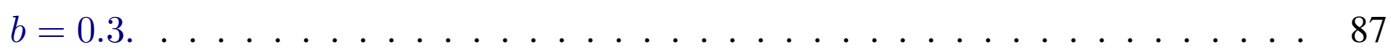

A.4 Empirical Distribution for $F_{12, t}\left(\tau_{j}\right)$ and $B_{12, t}\left(\tau_{j}\right)$ in Example 3.4 . . . . . . . . 88

A.5 PDC for Simulation 3 - Chapter $5 \ldots \ldots \ldots$

A.6 WTC for Simulation 3 - Chapter $5 \ldots \ldots \ldots$. . . . . . . . . . . . 89 
A.7 DWC between $X_{1, t}$ and $X_{2, t}$ for Simulation 3 - Chapter $5 \ldots \ldots \ldots \ldots$

A.8 DWC between $X_{1, t}$ and $X_{3, t}$ for Simulation 3 - Chapter $5 \ldots \ldots \ldots \ldots$

A.9 DWC between $X_{2, t}$ and $X_{3, t}$ for Simulation 3 - Chapter $5 \ldots \ldots \ldots$

A.10 pDWC between $X_{1, t}$ and $X_{2, t}$ for Simulation 3 - Chapter $5 \ldots \ldots$. . . . . . 93

A.11 pDWC between $X_{1, t}$ and $X_{3, t}$ for Simulation 3 - Chapter $5 \ldots \ldots \ldots$

A.12 pDWC between $X_{2, t}$ and $X_{3, t}$ for Simulation 3 - Chapter $5 \ldots \ldots \ldots$

A.13 ppDWC between $X_{1, t}$ and $X_{2, t}$ for Simulation 3 - Chapter $5 \ldots \ldots \ldots$

A.14 ppDWC between $X_{1, t}$ and $X_{3, t}$ for Simulation 3 - Chapter $5 \ldots \ldots \ldots$

A.15 ppDWC between $X_{2, t}$ and $X_{3, t}$ for Simulation 3 - Chapter $5 \ldots \ldots \ldots$

A.16 Standard Deviations for DWC in Simulation 1. . . . . . . . . . . . . . . . . . 99

A.17 Standard Deviations for DWC in Simulation 2. . . . . . . . . . . . . . 100

A.18 Standard Deviations for DWC in Simulation 3: $X_{1, t}$ and $X_{2, t} \ldots \ldots \ldots \ldots 1$

A.19 Standard Deviations for DWC in Simulation 3: $X_{1, t}$ and $X_{3, t} \ldots \ldots \ldots \ldots$

A.20 Standard Deviations for DWC in Simulation 3: $X_{2, t}$ and $X_{3, t} \ldots \ldots \ldots \ldots$

A.21 Standard Deviations for pDWC in Simulation 3: $X_{1, t}$ and $X_{2, t} \ldots \ldots \ldots$. . . . . 104

A.22 Standard Deviations for pDWC in Simulation 3: $X_{1, t}$ and $X_{3, t} \ldots \ldots \ldots \ldots$

A.23 Standard Deviations for pDWC in Simulation 3: $X_{2, t}$ and $X_{3, t} \ldots \ldots \ldots \ldots$

A.24 Standard Deviations for ppDWC in Simulation 3: $X_{1, t}$ and $X_{2, t} \ldots \ldots \ldots \ldots$

A.25 Standard Deviations for ppDWC in Simulation 3: $X_{1, t}$ and $X_{3, t} \ldots \ldots \ldots \ldots$

A.26 Standard Deviations for ppDWC in Simulation 3: $X_{2, t}$ and $X_{3, t} \ldots \ldots \ldots$

B.1 Regions of directed covariance . . . . . . . . . . . . . . . . . . . 121 


\section{List of Tables}

2.1 Examples of SDF for MA(1), AR(1) and $\mathrm{AR}(2)$ processes $\ldots \ldots \ldots \ldots$

4.1 Variation of values for $l$ and $l^{\prime}$ for term $B_{X_{1}, X_{2},-t}\left(\tau_{j}\right)$ in equation (4.9). . . . . . 53

4.2 Alternative form to write Table $4.1 \ldots \ldots \ldots \ldots \ldots$

6.1 Proportion of rejected tests for each period, scale and comparison for average of baseline trials $(\mathrm{S} 200)$ using DWC . . . . . . . . . . . . . . 71

6.2 Proportion of rejected tests for each period, scale and comparison for average of right movement trials $(\mathrm{S} 2+\mathrm{S} 20)$ using DWC . . . . . . . . . . . . . . 73

6.3 Proportion of rejected tests for each period, scale and comparison for average of left movement trials $(\mathrm{S} 1+\mathrm{S} 10)$ using DWC . . . . . . . . . . . . . . 73

6.4 Proportion of rejected tests for each period, scale and comparison for average of baseline trials $(\mathrm{S} 200)$ using $\mathrm{pDWC} \ldots \ldots \ldots \ldots$. . . . . . . . . . . . 75

6.5 Proportion of rejected tests for each period, scale and comparison for average of right movement trials $(\mathrm{S} 2+\mathrm{S} 20)$ using $\mathrm{pDWC} \ldots \ldots \ldots \ldots$

6.6 Proportion of rejected tests for each period, scale and comparison for average of left movement trials $(\mathrm{S} 1+\mathrm{S} 10)$ using $\mathrm{pDWC} \ldots \ldots \ldots \ldots$

B.1 Categories of relations between components of $\mathbf{X}_{t, T} \ldots \ldots \ldots \ldots \ldots$ 


\section{Chapter 1}

\section{Introduction}

Causal relations between non-stationary time series is a key topic in Neurosciences. By identifying causal relations, we can better understand the brain connectivity to propose more efficient treatments for diseases like Parkinson disease, epilepsy, schizophrenia and Alzheimer disease. Important methodologies like Directed Coherence (DC, Saito and Harashima (1981)), Partial Directed Coherence (PDC, Baccalá and Sameshima (2001)), Cross Wavelet Transform (XWT) and Wavelet Coherence (WTC, Torrence and Compo (1998) and Grinsted et al. (2004)) have been used and have largely contributed to study the origin and direction of signals. For this later, the basic idea is to consider the phase between power signals at the time-scale domain to suggest causality between the processes.

Consider a multivariate stationary process with $N$ components (univariate time series). Given two of its components, the main idea behind PDC is to estimate the relationship and functional connection between them by decomposing their covariance into backward and forward signals, after removed the influence of others. Takahashi et al. (2007) have shown that there is a direct relation between this concept and the Granger causality (Granger (1969)) definition in the frequency domain. PDC assumes this multivariate process can be represented by a Vector Autoregressive (VAR) model and uses its representation in the frequency domain to decompose the covariance function.

PDC has two factors, that will be detailed in next chapter: a feedforward, $\pi_{12}(\omega)$, and a feedback, $\pi_{21}(\omega)$. They represent, for a $N$-variate stationary time series $\mathbf{X}_{t}=\left[\begin{array}{llll}X_{1, t} & X_{2, t} & \ldots & X_{N, t}\end{array}\right]^{\top}$, the directed relation from $X_{1, t}$ to $X_{2, t}$ and vice-versa. Since they are calculated in the frequency domain, $\omega$ represents the frequency (i.e. cycles per unit of time) and its domain is $[-0.5,0.5]$. For instance, consider we have a trivariate process with the causal relations represented in Figure 1.1. PDC should be able to identify the relation between $X_{1}$ and $X_{2}$ components excluding the external influence of $X_{3}$. In other words, the estimation of $\pi_{12}(\omega)$ would be significant while $\pi_{21}(\omega)$ will appear almost inexistent. In situations with non-stationary time series, PDC can be estimated considering windows for locally stationary processes (Lopes (2014)). Milde et al. (2011) proposed a time-varying PDC, estimating a time-variant multivariate autoregressive(tv-MVAR) models instead of a VAR(p). We can estimate these models by Kalman filter (Arnold et al. (1998) and Pester et al. (2015)) or also by wavelets (Sato et al. (2007)).

In a stationary process, the statistical properties of the process do not change over time (Priestley (1981)). Non-stationary processes, on the other hand, fail to satisfy Wiener-Khintchine Theorem of Spectral Representation and therefore, Fourier Spectral Density Function is not expressed in its usual 


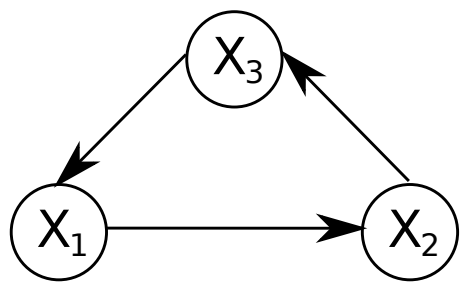

Figure 1.1. Diagram representing causal relations between components in a trivariate process.

form. In this case, it would be necessary to use the Evolutionary Spectrum.

When we analyze brain's activity, we must deal with non-stationarity situations like sudden pattern changes or local characteristics of the signals. These signals peculiarities might not fulfill the stationarity assumption in Fourier Analysis, especially in experiments with external stimuli.

For instance, consider the following example taken from Härdle et al. (1997):

Example 1.1 (Härdle et al. (1997)). Define $f(x)$ a step function:

$$
f(x)= \begin{cases}-1 & x \in[-1 / 2,0] \\ 1 & x \in(0,1 / 2]\end{cases}
$$

Despite of its simplicity, $f(x)$ is poorly approximated by Fourier expansion, which has the form:

$$
f(x)=\sum_{\substack{k=1 \\ \text { kodd }}}^{\infty} \frac{4}{\pi k} \sin (2 \pi k x)
$$

Figure 1.2 shows the approximation of (1.1) considering $K=5$ and $K=500$ coefficients of the Fourier expansion.
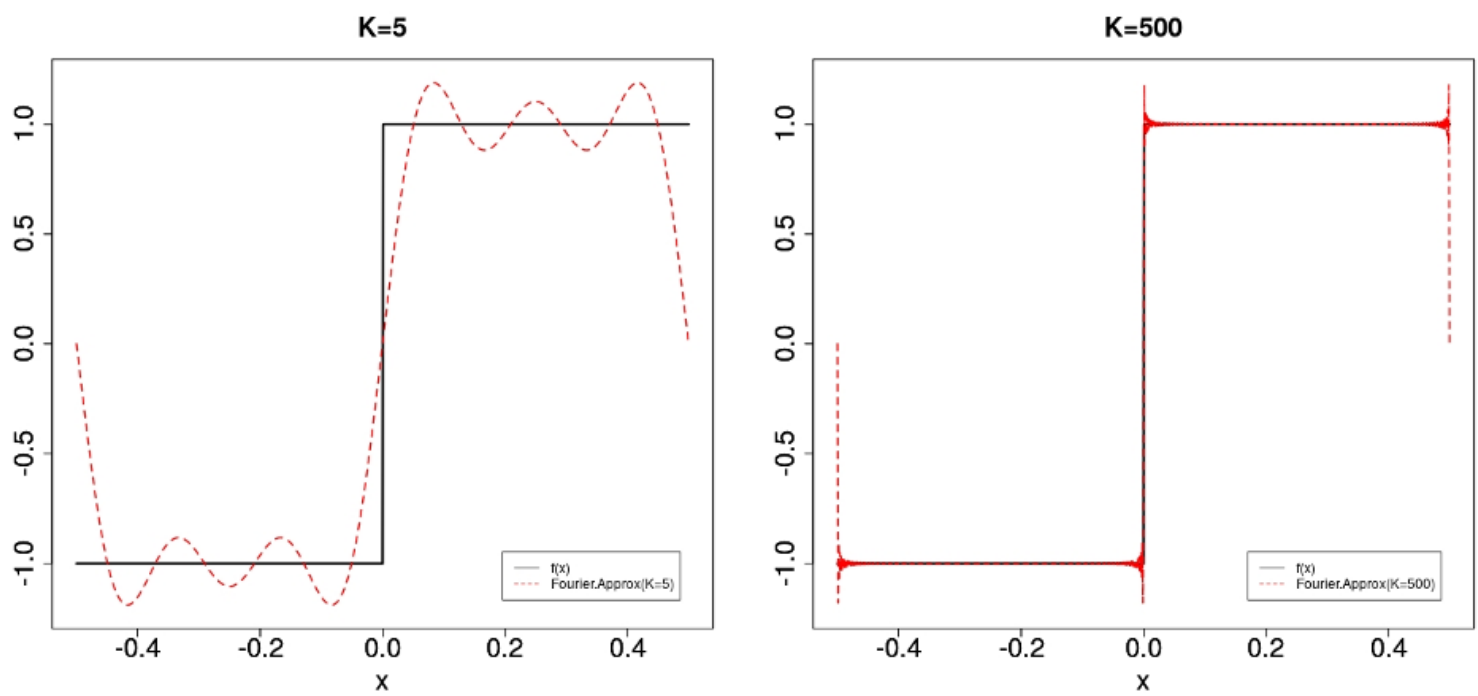

Figure 1.2. (Example 1.1 from Härdle et al. (1997)). Fourier approximation for step function in (1.1). Left graph uses $K=5$ Fourier coefficients and graph on the right uses $K=500$. 
As we can see, Fourier approximation cannot give a good approximation of the function (1.1), despite the number of used coefficients.

For these situations, wavelet analysis is more flexible and can identify local patterns and jumps with a small number of coefficients. In this case, using Haar wavelets,

$$
\psi(x)= \begin{cases}-1 & x \in[-1 / 2,0] \\ 1 & x \in(0,1 / 2]\end{cases}
$$

then the function (1.1) can be perfectly represented with two wavelet coefficients. See Section 2.4.

The main contribution of this thesis is to propose two decompositions of the Wavelet Covariance (Percival and Walden (2000)) for non-stationary time series and capture the causal relations between past and present observations: the Directed Wavelet Covariance (DWC) and the Partial Directed Wavelet Covariance (pDWC). For non-stationary time series, Wavelet Analysis is more appropriate than Fourier Analysis. Also, Wavelet Variance and Covariance have good properties of energy conservation and are indexed in the time-scale domain, which is useful to analyze the data with multiple smoothness degrees and identify local characteristics of the processes.

The DWC between two signals, $X_{t}$ and $Y_{t}$, is a decomposition of the Wavelet Covariance into two factors: forward (say, from $X_{t}$ to $Y_{t}$ ) and backward (from $Y_{t}$ to $X_{t}$ ). We proposed these quantities in wavelet domain i.e., they are indexed in time-scale. Thus, they can evaluate the relation between $X_{t}$ and $Y_{t}$ for different degrees of smoothness and also in different periods of time.

The pDWC measures the relation of two components in $\mathbf{X}_{t}=\left[X_{1, t}, \ldots, X_{N, t}\right]^{\top}$ after removing the impact of the other $N-2$ components. We propose two ways to measure that relation. In the first method, we filter the bivariate process $\left[X_{a, t}, X_{b, t}\right]^{\top}$ by removing the linear influence of the other $\mathbf{X}_{t}$

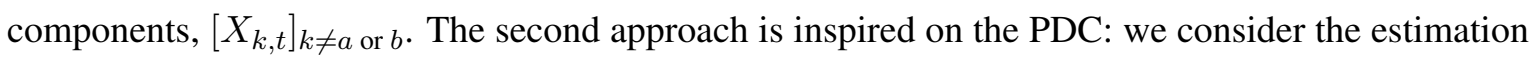
of the multivariate process $\mathbf{X}_{t}=\left[X_{1, t}, \ldots, X_{N, t}\right]^{\top}$ by an $N$-variate time-varying VAR (tv-VAR) and then we calculate the DWC between two components, say $X_{1}(t)$ and $X_{2}(t)$, eliminating the influence of the other components $X_{3}(t), \ldots, X_{N}(t)$.

We organized the thesis in three blocks. First, in Chapter two, we briefly review important concepts used in the work. We start with Spectral Analysis, which is the basis for the Partial Directed Coherence (Baccalá and Sameshima (2001)), presented afterward. We define the Discrete Fourier Transform, the Spectrum, and the Cross Spectrum. They are the representation of the variance and covariance of a (multivariate) stationary process in the frequency domain and are used to define the Coherence and Partial Coherence, also presented in the same section.

Then we present the wavelet background: the Continuous Wavelet Transform, the Discrete Wavelet Transform and the Maximal Overlap Discrete Wavelet Transform by using high-pass and low-pass filters, as presented in Percival and Walden (2000). One important characteristic of Wavelet Analysis is the multiresolution analysis, i.e., the capability of the wavelet transform to represent a process in multiple levels of resolution. Since the Wavelet Coherence (Grinsted et al. (2004)) will be compared with DWC, we also present its definition.

In Chapter 2 we also present the definition of Locally Stationary Processes (LSP) in the perspective 
of two authors: Priestley (1965) and Dahlhaus (1996). For this thesis we will use Dahlhaus' definition of LSP, which considers that increasing the sample size improves the process' local information. These two chapters are the main contribution of this work.

In the second block, Chapters three and four, we propose the Directed Wavelet Covariance and its properties, followed by two different concepts of partial Directed Wavelet Covariance and their properties.

Finally, in the third part, we start simulating processes and comparing the results between PDC, WTC, DWC and pDWC in Chapter five. Chapter six is about data analysis. We present conclusions for a real EEG database based on DWC's and pDWC's outputs. Finally in Chapter seven we discuss the results and limitations of the DWC and pDWC and propose future studies. 


\section{Chapter 2}

\section{Methods}

In this chapter we review important techniques used to propose the Directed Wavelet Covariance (DWC) in Chapter 3. First we present key concepts involving spectral analysis and Fourier transforms. We define the Fourier Transform of processes with discrete time (FT), the Spectral Density Function, and the Evolutionary Spectrum for locally stationary processes (see Section 2.3). Then we present the Partial Directed Coherence (PDC, Baccalá and Sameshima (2001)), a causal estimator in the frequency domain, and the Wavelet Coherence (WTC, Torrence and Compo (1998)), a correlation measure between processes in the wavelet domain. Both concepts will be compared with the proposed Directed Wavelet Covariance in Chapter 5. Finally, we present the definition of Locally Stationary Processes adopted for the next chapters and an overview of wavelet theory.

\subsection{Spectral Analysis}

Spectral Analysis is firstly related to the works of Jean Baptiste Fourier (1822) about heat transfer. It focus on representing periodic functions, say $f(t)$, with finite energy, $\sum_{t} f(t)^{2}<\infty$, by a linear combination of sines and cosines. In this section we briefly introduce key concepts from Fourier theory in the context of stochastic processes and time series. Priestley (1981) and Percival and Walden (1993) present a detailed discussion on spectral analysis for stochastic processes.

\subsubsection{Fourier Transform of Processes with Discrete Time}

Let $\left\{a_{t}, t \in \mathbb{Z}\right\}$ be an infinite sequence of real or complex values with finite energy, i.e., $\sum_{t}\left|a_{t}\right|^{2}<$ $\infty$. The Fourier Transform (FT) of $\left\{a_{t}\right\}$ is defined as

$$
A(\omega)=\sum_{t=-\infty}^{\infty} a_{t} e^{-i 2 \pi \omega t}
$$

where $\omega \in \mathbb{R}$ is known as frequency. Percival and Walden (2000) describe the frequency in terms of the Euler relationship,

$$
e^{-i 2 \pi \omega u}=\cos (2 \pi \omega u)-i \cdot \sin (2 \pi \omega u),
$$

as "how many cycles the real and imaginary components of the function each go through as $u$ sweeps from 0 to $1 " . A(\omega)$ is therefore the representation in the frequency domain of $\left\{a_{t}\right\}$. The inverse 
operation is given by:

$$
a_{t}=\int_{-1 / 2}^{1 / 2} A(\omega) e^{i 2 \pi \omega t} d \omega
$$

Some important properties of $A(\omega)$ are:

- $A(\omega+j)=A(\omega)$ for any integer $j$;

- if $\left\{a_{t}\right\}$ is real-valued then $A(-\omega)=A^{*}(\omega)$, where $A^{*}(\omega)$ is the conjugate of $A(\omega)$;

The FT is a transformation that preserves the sequence's energy. Parseval's theorem says that if $\left\{a_{t}\right\}$ and $\left\{b_{t}\right\}$ are two sequences with finite energy, $\sum_{t}\left|a_{t}\right|^{2}<\infty$ and $\sum_{t}\left|b_{t}\right|^{2}<\infty$, and $A(\omega)$ and $B(\omega)$ are their respective FTs, then

$$
\begin{aligned}
\sum_{t=-\infty}^{\infty}\left|a_{t}\right|^{2} & =\int_{-1 / 2}^{1 / 2}|A(\omega)|^{2} d \omega, \\
\sum_{t=-\infty}^{\infty} a_{t} b_{t}^{*} & =\int_{-1 / 2}^{1 / 2} A(\omega) B^{*}(\omega) d \omega .
\end{aligned}
$$

This result is important in the definition of the Partial Directed Coherence (PDC) in next section. As we will show later, PDC is a decomposition of the cross spectrum and therefore, is calculated in the frequency domain. Without the energy preservation property, PDC would not represent the causal relation between sampled signals in time domain.

The representation of finite sequences $\left\{a_{t} \mid t=0, \ldots, T-1\right\}$ is a direct result of (2.1) if we consider the $a_{t}=0$ for $t \leq-1$ and $t \geq T$. Formally, for $\left\{a_{t} \mid t=0, \ldots, T-1\right\}$ a finite sequence with real or complex values, the Discrete Fourier Transform (DFT) is a sequence $\left\{A_{k}\right\}$ given by

$$
A_{k}=\sum_{t=0}^{T-1} a_{t} e^{-i 2 \pi t \frac{k}{T}}, k=0, \ldots, T-1,
$$

which is associated to the frequency $\omega_{k}=k / T$. The inverse relation is

$$
a_{t}=\sum_{t=0}^{T-1} A_{k} e^{i 2 \pi t \frac{k}{T}}, t=0, \ldots, T-1 .
$$

$a_{t}$ is commonly known as Inverse DFT of $\left\{A_{k}\right\}$. Parseval's theorem is also valid for finite sequences and is given by

$$
\sum_{t=0}^{T-1}\left|a_{t}\right|^{2}=\frac{1}{N} \sum_{t=0}^{T-1}\left|A_{k}\right|^{2} .
$$

\subsubsection{Spectral Density Function and Cross Spectrum}

We now focus on stationary stochastic processes $\left\{X_{t}, t \in \mathbb{Z}\right\}$. Stationarity is defined as follows. 
Definition 2.1. The process $\left\{X_{t}\right\}$ is said to be strictly stationary if, for any admissible $t_{1}, t_{2}, \ldots, t_{n}$, and any $k$, the joint probability distribution of $\left\{X_{t_{1}}, X_{t_{2}}, \ldots, X_{t_{n}}\right\}$ is identical with the probability distribution of $\left\{X_{t_{1}+k}, X_{t_{2}+k}, \ldots, X_{t_{n}+k}\right\}$, i.e. if

$$
F\left(X_{t_{1}}, \ldots, X_{t_{n}}\right)\left(x_{1}, \ldots, x_{n}\right)=F\left(X_{t_{1}+k}, \ldots, X_{t_{n}+k}\right)\left(x_{1}, \ldots, x_{n}\right)
$$

where $F($.$) denotes the distribution function of the set of random variables which appear as suffixes.$

Definition 2.2. The process $\left\{X_{t}\right\}$ is said to be stationary up to order $\mathrm{m}$ if, for any admissible $t_{1}, t_{2}, \ldots, t_{n}$, and any $k$, all the joint moments up to order $\mathrm{m}$ of $\left\{X_{t_{1}}, X_{t_{2}}, \ldots, X_{t_{n}}\right\}$ exist and equal the corresponding joint moments up to order $\mathrm{m}$ of $\left\{X_{t_{1}+k}, X_{t_{2}+k}, \ldots, X_{t_{n}+k}\right\}$.

We often consider $k=2$ and the process is called Weakly Stationary or Second-order Stationary. In this case:

- $\mathbb{E}\left(X_{t}\right)=\mu$ is constant for all $t$;

- $\mathbb{E}\left(X_{t}^{2}\right)=\mu_{2}$ is constant for all $t$. Therefore, $\operatorname{Var}\left(X_{t}\right)=\mu_{2}-\mu=\sigma^{2}$ is also constant;

- For any $s, t$ with $s \neq t, \mathbb{E}\left(X_{t} X_{s}\right)$ depends only on $|t-s|$. Hence, $\operatorname{cov}\left(X_{t}, X_{s}\right)$ also depends only on $|t-s|$.

Notice that stationarity is not only a property of univariate processes. Brillinger (2001) define the multivariate stationary process as:

Definition 2.3 (Brillinger (2001)). An $r$-dimensional vector series $\mathbf{X}_{t}, t \in \mathbb{Z}$ is called Second-order Stationary if

- $\mathbb{E}\left(\mathbf{X}_{t}\right)=\boldsymbol{\mu}$ is constant for all $t$;

- Let $X_{a, t}$ and $X_{b, t}$ two components of $\mathbf{X}_{t}, a, b=1, \ldots, r$. Then $\operatorname{Cov}\left(X_{a, t+u}, X_{b, t}\right)=\gamma_{a, b}(u)$, for $t, u \in \mathbb{Z}$. Eventually, if $a=b$ then $\operatorname{Cov}\left(X_{a, t+u}, X_{a, t}\right)=\gamma_{a}(u)$.

Let now $\left\{X_{t}, t \in \mathbb{Z}\right\}$ be a stationary stochastic process with $\gamma_{X}$ its covariance function such that $\sum_{u=-\infty}^{\infty}\left|\gamma_{X}(u)\right|<\infty$. The Spectral Density Function (SDF) of $\left\{X_{t}\right\}$ is given by the Fourier transform of its covariance function, i.e.

$$
f_{X}(\omega)=\sum_{u=-\infty}^{\infty} \gamma(u) e^{-i 2 \pi \omega u},-1 / 2<\omega<1 / 2,
$$

where $\gamma(\tau)=\operatorname{Cov}\left(X_{t}, X_{t+\tau}\right), t, \tau \in \mathbb{Z}$, such that the inverse Fourier transform of $f_{X}(\omega)$ results in

$$
\gamma(u)=\int_{-1 / 2}^{1 / 2} f_{X}(\omega) e^{i 2 \pi \omega u} d \omega
$$

Example 2.1 (Morettin (2014)). We consider the three stationary processes presented in Table 2.1. Figure 2.1 shows the SDF for stationary processes MA(1), AR(1), and AR(2). Notice that these three 
processes have very distinct characteristics. The MA(1) process presents characteristics of a highpass filter while $\operatorname{AR}(1)$ process has, in this case $(\phi=0.5)$, characteristics of a low-pass filter. The $\mathrm{AR}(2)$ process is also known as a pseudo periodic process, given its "false" periodicity.

$\mathrm{MA}(1): \theta=0.8$

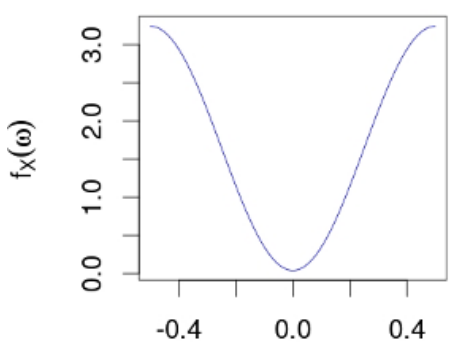

$\omega$
$\operatorname{AR}(1): \phi=0.5$

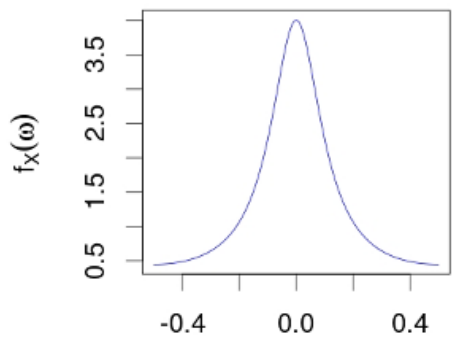

$\omega$
$\operatorname{AR}(2): \phi_{1}=1.5, \phi_{2}=-0.92$

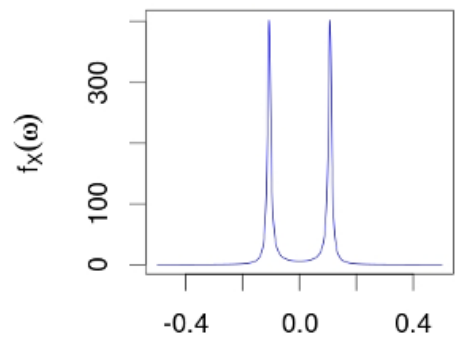

$\omega$

Figure 2.1. Examples of Spectral Density Functions (Morettin (2014)).

The Spectrum in (2.3) is defined for univariate time series. If we have a multivariate time series $\mathbf{X}_{t}=\left(X_{1, t}, X_{2, t}, \ldots, X_{N, t}\right)^{\top}$, we can also define the Cross Spectrum between two components of $\mathbf{X}_{t}$. To define the Cross Spectrum, let the cross-covariance function between two components $X_{i, t}$ and $X_{j, t}$ of $\mathbf{X}_{t}$ be

$$
\gamma_{i, j}(k)=\operatorname{cov}\left(X_{i, t}, X_{j, t+k}\right), k=0, \pm 1, \pm 2, \ldots, i \neq j \text { and } i, j=1, \ldots, N
$$

and assume that this quantity exists and is absolutely summable, i.e., $\sum_{k}\left|\gamma_{i, j}(k)\right|<\infty$. Then the Cross Spectrum $f_{i, j}(\omega)$ is given by

$$
f_{i, j}(\omega)=\sum_{k=-\infty}^{\infty} \gamma_{i, j}(k) e^{-i 2 \pi \omega k},-\pi<\omega<\pi, i \neq j \text { and } i, j=1, \ldots, N
$$

(2.3) and (2.4) together compose the spectral representation of $\mathbf{X}_{t}$ 's energy in the frequency domain

\section{Process Definition}

\section{Covariance Function}

SDF

MA(1) $X_{t}=\theta \epsilon_{t-1}+\epsilon_{t} \quad\left\{\begin{array}{ll}1+\theta^{2}, & h=0 \\ \theta, & |h|=1 \\ 0, & \text { otherwise. }\end{array} \quad\left(1+\theta^{2}-2 \theta(\cos (2 \pi \omega))\right)\right.$

AR(1) $\quad X_{t}=\phi X_{t-1}+\epsilon_{t} \quad\left\{\begin{array}{ll}1 /\left(1+\phi^{2}\right), & h=0 \\ \phi^{|h|} /\left(1+\phi^{2}\right), & |h| \geq 1 .\end{array} \quad\left(1+\phi^{2}-2 \phi \cos (2 \pi \omega)\right)^{-1}\right.$

$\mathbf{A R}(2) \quad X_{t}=\sum_{i=1}^{2} \phi_{i} X_{t-i}+\epsilon_{t} \quad$ Recursively $\quad\left|1-\phi_{1} e^{-i 2 \pi \omega}-\phi_{2} e^{-i 4 \pi \omega}\right|^{-2}$

Table 2.1. Examples of SDF for MA(1), AR(1) and AR(2) processes from Morettin (2014). In all definitions, consider $\epsilon_{t} \sim W N(0,1)$, i.e. $\mathbb{E}\left(\epsilon_{t}\right)=0$, $\operatorname{var}\left(\epsilon_{t}\right)=1, \forall t$, and $\operatorname{cov}\left(\epsilon_{t}, \epsilon_{s}\right)=0, s \neq t$. 
and can be represented as the Cross Spectra Matrix, namely:

$$
\mathbf{f}(\omega)=\left[\begin{array}{ccc}
f_{11}(\omega) & \ldots & f_{1 N}(\omega) \\
\vdots & \ddots & \vdots \\
f_{N 1}(\omega) & \ldots & f_{N N}(\omega)
\end{array}\right]
$$

with $f_{i i}(\omega), i=1, \ldots, N$ the univariate spectrum for each component (2.3) and $f_{i, j}(\omega), i \neq j$ the cross spectrum between $X_{i, t}$ and $X_{j, t}$ defined in (2.4).

\subsubsection{Coherence and Partial Coherence}

The Coherence function is a version of the linear correlation function in the frequency domain. Since the Spectrum (2.3) and the Cross Spectrum (2.4) are the covariance and cross-covariance representation in the frequency domain, respectively, then it is intuitive defining the Coherence Function between two time series $X_{t}$ and $Y_{t}$ as

$$
\kappa_{X, Y}^{2}(\omega)=\frac{\left|f_{X, Y}(\omega)\right|^{2}}{f_{X}(\omega) f_{Y}(\omega)}
$$

with $f_{X, Y}(\omega)$ being the cross spectrum between $X_{t}$ and $Y_{t}$ and $f_{X}(\omega)$ and $f_{Y}(\omega)$ their spectra. One important feature of the $\kappa_{X, Y}^{2}(\omega)$ is $0 \leq \kappa_{X, Y}^{2}(\omega) \leq 1$ and this is consequence of the fact $\left|f_{X, Y}(\omega)\right|^{2} \leq f_{X}(\omega) \cdot f_{Y}(\omega)$.

Consider now a multivariate time series $\mathbf{Z}_{t}$ :

$$
\mathbf{Z}_{t}=\left[\begin{array}{c}
Y_{1, t} \\
\vdots \\
Y_{s, t} \\
X_{1, t} \\
\vdots \\
X_{r, t}
\end{array}\right]=\left[\begin{array}{c}
\mathbf{Y}_{t} \\
\mathbf{X}_{t}
\end{array}\right], t \in \mathbb{Z}
$$

with the mean and covariance matrices of $\left[\mathbf{Y}_{t}, \mathbf{X}_{t}\right]^{\top}$ given by

$$
\mathbb{E}\left[\begin{array}{c}
\mathbf{Y}_{t} \\
\mathbf{X}_{t}
\end{array}\right]=\left[\begin{array}{c}
\boldsymbol{\mu}_{Y} \\
\boldsymbol{\mu}_{X}
\end{array}\right] \quad, \quad \operatorname{Var}\left(\mathbf{Z}_{t}\right)=\left[\begin{array}{cc}
\Sigma_{Y Y} & \Sigma_{Y X} \\
\Sigma_{X Y} & \Sigma_{Y Y}
\end{array}\right]
$$

Suppose we want to calculate the covariance between two components of $\mathbf{Y}_{t}$, say $Y_{a, t}$ and $Y_{b, t}$, after removing the effect of the components of $\mathbf{X}_{t}$. Without loss of generality consider $a=1, b=2$ and $s=2$. Otherwise, rearrange $\mathbf{Z}_{t}$ to get the two time series as the first components. We aim to find an $s \times r$ linear filter $\{\mathbf{A}(u)\}$ and an $s$-dimension vector $\boldsymbol{\mu}$ such that

$$
\mathbb{E}\left\{\left[\mathbf{Y}_{t}-\boldsymbol{\mu}-\mathbf{A} \mathbf{X}_{t}\right]\left[\mathbf{Y}_{t}-\boldsymbol{\mu}-\mathbf{A} \mathbf{X}_{t}\right]^{\top}\right\}
$$


is minimum. In this case we have $s=2$ and it represents the dimension of $\mathbf{Y}_{t}$.

Theorem 2.1 (Brillinger (2001)). Let an $(r+s)$ vector-valued variate of the form (2.7) with mean and covariance matrices given by (2.8). Suppose $\Sigma_{X X}$ is nonsingular. Then $\boldsymbol{\mu}$ and $\mathbf{A}$ minimizing (2.9) are given by

$$
\boldsymbol{\mu}=\boldsymbol{\mu}_{Y}-\Sigma_{Y X} \Sigma_{X X}^{-1} \boldsymbol{\mu}_{X}
$$

and

$$
\mathbf{A}=\Sigma_{Y X} \Sigma_{X X}^{-1}
$$

The minimum achieved is

$$
\Sigma_{Y Y}-\Sigma_{Y X} \Sigma_{X X}^{-1} \Sigma_{X Y}
$$

and the variate

$$
\boldsymbol{\mu}_{Y}+\Sigma_{Y X} \Sigma_{X X}^{-1}\left(\mathbf{X}_{t}-\boldsymbol{\mu}_{X}\right)
$$

is called the best linear predictor of $\mathbf{Y}_{t}$ based on $\mathbf{X}_{t}$.

From Theorem 2.1 we define the process $\epsilon_{t}$ by:

$$
\boldsymbol{\epsilon}_{t}=\mathbf{Y}_{t}-\left(\boldsymbol{\mu}_{Y}+\Sigma_{Y X} \Sigma_{X X}^{-1}\left(\mathbf{X}_{t}-\boldsymbol{\mu}_{X}\right)\right), t \in \mathbb{Z}
$$

This is the residual process after filtering $\mathbf{Y}_{t}$ with the best linear filter based on $\mathbf{X}_{t}$ and has covariance matrix given by:

$$
\Sigma_{\epsilon \epsilon}=\Sigma_{Y Y}-\Sigma_{Y X} \Sigma_{X X}^{-1} \Sigma_{X Y} .
$$

The covariance of $\epsilon_{j}$ and $\epsilon_{k}, j, k \leq s$ is called the partial covariance of $Y_{j}$ and $Y_{k}$ after removing the linear influence of $\mathbf{X}_{t}$.

Now consider the following covariance functions and their respective spectra

$$
\begin{aligned}
& \operatorname{Cov}\left[Y_{1, t}, Y_{1, t+u}\right]=\gamma_{Y_{1}, Y_{1}}(u) \quad \Rightarrow \quad f_{Y_{1}, Y_{1}}(\omega)=\sum_{u} \gamma_{Y_{1}, Y_{1}}(u) e^{-i 2 \pi u \omega}, \\
& \operatorname{Cov}\left[Y_{2, t}, Y_{2, t+u}\right]=\gamma_{Y_{2}, Y_{2}}(u) \quad \Rightarrow \quad f_{Y_{2}, Y_{2}}(\omega)=\sum_{u} \gamma_{Y_{2}, Y_{2}}(u) e^{-i 2 \pi u \omega}, \\
& \operatorname{Cov}\left[\mathbf{X}_{\mathbf{t}}(u)\right]=\mathbf{C}_{\mathbf{X}}(u)=\left[\begin{array}{ccc}
\gamma_{X_{1}, X_{1}}(u) & \ldots & \gamma_{X_{1}, X_{r}}(u) \\
\vdots & \ddots & \vdots \\
\gamma_{X_{r}, X_{1}}(u) & \ldots & \gamma_{X_{r}, X_{r}}(u)
\end{array}\right] \Rightarrow \mathbf{f}_{\mathbf{X}}(\omega)=\sum_{u} \mathbf{C}_{X}(u) e^{-i 2 \pi u \omega},
\end{aligned}
$$




$$
\begin{aligned}
& \operatorname{Cov}\left[Y_{1, t}, \mathbf{X}_{\mathbf{t}}(u)\right]=\mathbf{C}_{Y_{1}, \mathbf{X}}(u)=\left[\begin{array}{c}
\gamma_{1,1}(u) \\
\vdots \\
\gamma_{1, r}(u)
\end{array}\right] \Rightarrow \mathbf{f}_{Y_{1}, \mathbf{X}}(\omega)=\sum_{u} \mathbf{C}_{Y_{1}, \mathbf{X}}(u) e^{-i 2 \pi u \omega}, \\
& \operatorname{Cov}\left[Y_{2, t}, \mathbf{X}_{\mathbf{t}}(u)\right]=\mathbf{C}_{Y_{2}, \mathbf{X}}(u)=\left[\begin{array}{c}
\gamma_{2,1}(u) \\
\vdots \\
\gamma_{2, r}(u)
\end{array}\right] \Rightarrow \mathbf{f}_{Y_{2}, \mathbf{X}}(\omega)=\sum_{u} \mathbf{C}_{Y_{2}, \mathbf{X}}(u) e^{-i 2 \pi u \omega}
\end{aligned}
$$

The Partial Cross Spectrum between $Y_{1, t}$ and $Y_{2, t}$ after removing the effects of $\mathbf{X}_{\mathbf{t}}=\left\{X_{k, t}, k=\right.$ $1, \ldots, r\}$, is given by:

$$
f_{Y_{1}, Y_{2} / X}(\omega)=f_{Y_{1}, Y_{2}}(\omega)-\mathbf{f}_{Y_{1}, X}(\omega) \mathbf{f}_{X}(\omega)^{-1} \mathbf{f}_{Y_{2}, \mathbf{X}}^{\top}(\omega)
$$

Yet, $Y_{1, t}$ and $Y_{2, t}$ may be related indirectly by some influence of $\mathbf{X}_{\mathbf{t}}$. Thus, to calculate the Partial Coherence, we also must exclude the influence of $\mathbf{X}_{\mathbf{t}}$ in each $Y_{1, t}$ and $Y_{2, t}$ separately. Hence, we calculate the spectrum of each component excluding the dependency on $\mathbf{X}_{\mathbf{t}}$ and then compute the Partial Spectra (Bloomfield (2000)), namely:

$$
\begin{aligned}
& {\left[\begin{array}{ll}
f_{Y_{1}, Y_{1} / X}(\omega) & f_{Y_{1}, Y_{2} / X}(\omega) \\
f_{Y_{2}, Y_{1} / X}(\omega) & f_{Y_{2}, Y_{2} / X}(\omega)
\end{array}\right]=\left[\begin{array}{ll}
f_{Y_{1}, Y_{1}}(\omega) & f_{Y_{1}, Y_{2}}(\omega) \\
f_{Y_{2}, Y_{1}}(\omega) & f_{Y_{2}, Y_{2}}(\omega)
\end{array}\right]-}
\end{aligned}
$$

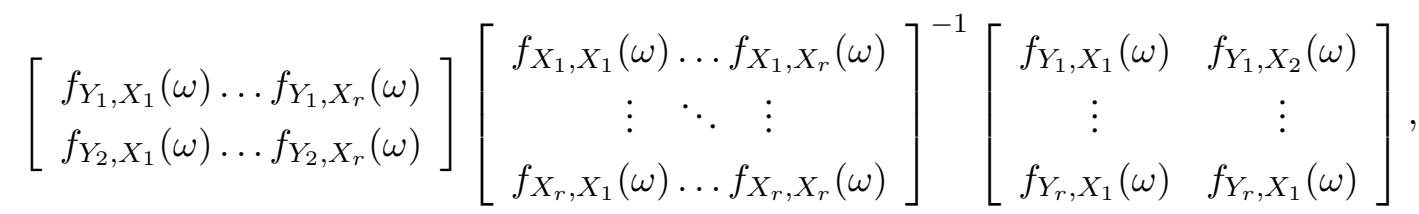

which allow us to define the Partial Coherence function between $Y_{1, t}$ and $Y_{2, t}$ as:

$$
\mathcal{K}_{Y_{1}, Y_{2} / X}(\omega)=\frac{f_{Y_{1}, Y_{2} / X}(\omega)}{\left[f_{Y_{1}, Y_{1} / X}(\omega) f_{Y_{2}, Y_{2} / X}(\omega)\right]^{1 / 2}} .
$$

Notice the similarity with the Coherence Function in (2.6), except that now we have the partial spectra involved whereas there we have the usual spectra.

\subsection{Partial Directed Coherence}

Directed Coherence (DC, Saito and Harashima (1981)) is equivalent to the bivariate case of Partial Directed Coherence (PDC, Baccalá and Sameshima (2001)) . Saito and Harashima (1981) proposed the Directed Coherence as an energy decomposition of a process based on its representation as a vector autoregressive model. The main idea behind PDC (and therefore DC) is to estimate the structural relationship and functional connection between time series by decomposing the variability and covariance of these processes in feedforward and feedback aspects. Takahashi et al. (2007) have shown that there is a direct relation between these concepts and the Granger causality definition in the frequency domain. In this section, we will present the theory behind PDC and the results for DC are similar, since it is a particular case of PDC. 
Consider a zero-mean, multivariate stationary process $\mathbf{X}_{t}=\left(X_{1, t}, \ldots, X_{N, t}\right)^{\top}, t \in \mathbb{Z}$ such that:

$$
\mathbf{X}_{t}=\sum_{r=1}^{p} \mathbf{A}_{r} \mathbf{X}_{t-r}+\varepsilon_{t}, t \in\{1,2, \ldots T\}
$$

with

$$
\mathbf{A}_{r}=\left[\begin{array}{ccc}
a_{11}(r) & \ldots & a_{1 N}(r) \\
\vdots & \ddots & \vdots \\
a_{N 1}(r) & \ldots & a_{N N}(r)
\end{array}\right]
$$

and $\varepsilon_{t}=\left(\varepsilon_{1, t}, \ldots, \varepsilon_{N, t}\right)^{\top}$ with $\varepsilon_{1, t}, \ldots, \varepsilon_{N, t}$ identically distributed with $\mathbb{E}\left(\varepsilon_{i, t}\right) \equiv 0, \mathbb{E}\left(\varepsilon_{i, s} \varepsilon_{i, t}\right) \equiv 0$ for $s \neq t, \mathbb{E}\left(\varepsilon_{i, t}^{2}\right) \equiv \sigma_{i}^{2}, i=1, \ldots, N$.

The coefficients $a_{i j}(r)$ estimate the linear interaction between $X_{j, t-r}$ and $X_{i, t}, i, j=1, \ldots, N$. The $p$-th order for the model might be given by AIC or BIC criterion.

Also consider the cross spectra (2.17) and its general spectral factorization (2.18):

$$
\begin{aligned}
\mathbf{f}(\omega) & =\left[\begin{array}{ccc}
f_{11}(\omega) & \ldots & f_{1 N}(\omega) \\
\vdots & \ddots & \vdots \\
f_{N 1}(\omega) & \ldots & f_{N N}(\omega)
\end{array}\right] \\
\mathbf{f}(\omega) & =\mathbf{H}(\omega) \boldsymbol{\Sigma} \mathbf{H}^{H}(\omega),
\end{aligned}
$$

$f_{i j}(\omega), i, j=1, \ldots, N$ are the Fourier cross spectra between components $i$ and $j, \mathbf{H}^{H}($.$) repre-$ sents the Hermitian matrix of $\mathbf{H}(),. \boldsymbol{\Sigma}$ is the covariance matrix of lag zero, $\boldsymbol{\Sigma}=\left[\gamma_{i j}(0)\right], \gamma_{i j}(0)=$ $\operatorname{Cov}\left(X_{i, t}, X_{j, t}\right), i, j=1, \ldots, N$ and $t=1, \ldots, T$ for discrete processes or $t \in[0, T]$ for continuous processes.

The matrix $\mathbf{H}(\omega)$ is given by

$$
\mathbf{H}(\omega)=\overline{\mathbf{A}}^{-1}(\omega),
$$

with

$$
\begin{aligned}
\overline{\mathbf{A}} & =\mathbf{I}-\mathbf{A}(\omega), \\
\mathbf{A}(\omega) & =\left.\sum_{r=1}^{p} \mathbf{A}_{r} z^{-r}\right|_{z=e^{-i 2 \pi \omega}}
\end{aligned}
$$

with the following Partial Coherence Function (2.15) factorization:

$$
\mathcal{K}_{i, j / X}(\omega)=\frac{\overline{\mathbf{a}}_{i}^{H}(\omega) \Sigma^{-1} \overline{\mathbf{a}}_{j}(\omega)}{\sqrt{\left(\overline{\mathbf{a}}_{i}^{H}(\omega) \Sigma^{-1} \overline{\mathbf{a}}_{i}(\omega)\right)\left(\overline{\mathbf{a}}_{j}^{H}(\omega) \Sigma^{-1} \overline{\mathbf{a}}_{j}(\omega)\right)}} .
$$


Baccalá and Sameshima (2001) define The Partial Directed Coherence (PDC) factors between $X_{i, t}$ and $X_{j, t}$ as

$$
\pi_{i j}(\omega):=\frac{\bar{A}_{i j}(\omega)}{\sqrt{\overline{\mathbf{a}}_{j}^{H}(\omega) \overline{\mathbf{a}}_{j}(\omega)}}, \quad \pi_{j i}(\omega):=\frac{\bar{A}_{j i}(\omega)}{\sqrt{\overline{\mathbf{a}}_{i}^{H}(\omega) \overline{\mathbf{a}}_{i}(\omega)}},
$$

where $\overline{\mathbf{a}}_{j}(\omega)$ and $\bar{A}_{i j}(\omega)$ are, respectively, $j$-th column and the (i,j)-th element of $\overline{\mathbf{A}}(\omega), i, j=$ $1, \ldots, N$.

These factors evaluate the influence of $X_{i, t}$ on $X_{j, t}$ and vice-versa, separately, excluding the effect of the other components of $\mathbf{X}_{t}$. Since they are calculated in the frequency domain, they also measure in which frequencies the relations are significant and also compute the phases delays between the processes.

To exemplify its effectiveness, we present an example extracted from Baccalá and Sameshima (2001).

Example 2.2 (Baccalá and Sameshima (2001)). Consider the stationary VAR(1) given by:

$$
\left[\begin{array}{l}
x_{1, t} \\
x_{2, t} \\
x_{3, t}
\end{array}\right]=\left[\begin{array}{rrr}
0.5 & 0.3 & 0.4 \\
-0.5 & 0.3 & 1 \\
0 & -0.3 & -0.2
\end{array}\right] \cdot\left[\begin{array}{l}
x_{1, t-1} \\
x_{2, t-1} \\
x_{3, t-1}
\end{array}\right]+\left[\begin{array}{l}
\varepsilon_{1, t} \\
\varepsilon_{2, t} \\
\varepsilon_{3, t}
\end{array}\right],
$$

with $\varepsilon_{i, t} \sim W N(0,1), i=1,2,3$. We simulate this process and Figure 2.2 shows the three simulated components. Despite of no visual evidence that the three time series are correlated, the dependency created in (2.20) becomes evident with the calculated PDC, presented in Figure 2.3.

The PDC graphs are proportional to the simulated directed relation, caused by the coefficients of the model (2.20). For instance, the PDC relation with largest values is $\pi_{2,3}$, that coincides with the largest coefficient in absolute value, $a_{2,3}=1$. Since PDC is calculated in the frequency domain, we can also conclude that the relations of $X_{1}$ and $X_{2}$ with their past, measured by $\pi_{1,1}$ and $\pi_{2,2}$, occur more often in high frequencies, whereas relations in $\pi_{1,2}, \pi_{1,3}$ and $\pi_{2,3}$ have more energy concentrated in low frequencies.

However, because Fourier analysis requires stationary processes, Partial Directed Coherence may fail for non-stationary processes. Lopes (2014) calculated the PDC assuming local stationarity (see Section 2.3 below) of the processes and evaluated their causal relations in local regions using a moving window of width $M$. With a particular value $M$, the estimated vector of PDCs $\left(\mathbf{R}_{X_{i, t}, X_{j, t}}(\omega)\right)$ for the sample $\{1, \ldots, T\}$ is:

$$
\mathbf{R}_{X_{i, t}, X_{j, t}}(\omega)=\left[\left.\mathbf{r}_{0, t}(\omega)\right|_{t \in J_{0}}, \ldots,\left.\mathbf{r}_{K-1, t}(\omega)\right|_{t \in J_{K-1}}\right]_{\left[\frac{M}{2}\right] \times K},
$$

where $M$ is the window width, $\mathbf{J}=\left\{\left(J_{k}\right), k=0, \ldots, K-1\right\}$ is a set of intervals such that $\bigcup_{k=0}^{K-1} J_{k}=\{1, \ldots, T\}$ and $\mathrm{K}=\left[\frac{T}{M}\right]$ (largest integer less or equal than $\frac{T}{M}$ ).

Each $\left.\mathbf{r}_{k, t}(\omega)\right|_{t \in J_{k}}, k=0, \ldots, K-1$ estimates the PDC between $X_{1, t}$ and $X_{2, t}$ considering $t \in J_{k}$ as an isolated subset with no influence from the others. $\left(\mathbf{R}_{X_{j, t}, X_{i, t}}(\omega)\right)$ over $\{1, \ldots, T\}$ is defined 


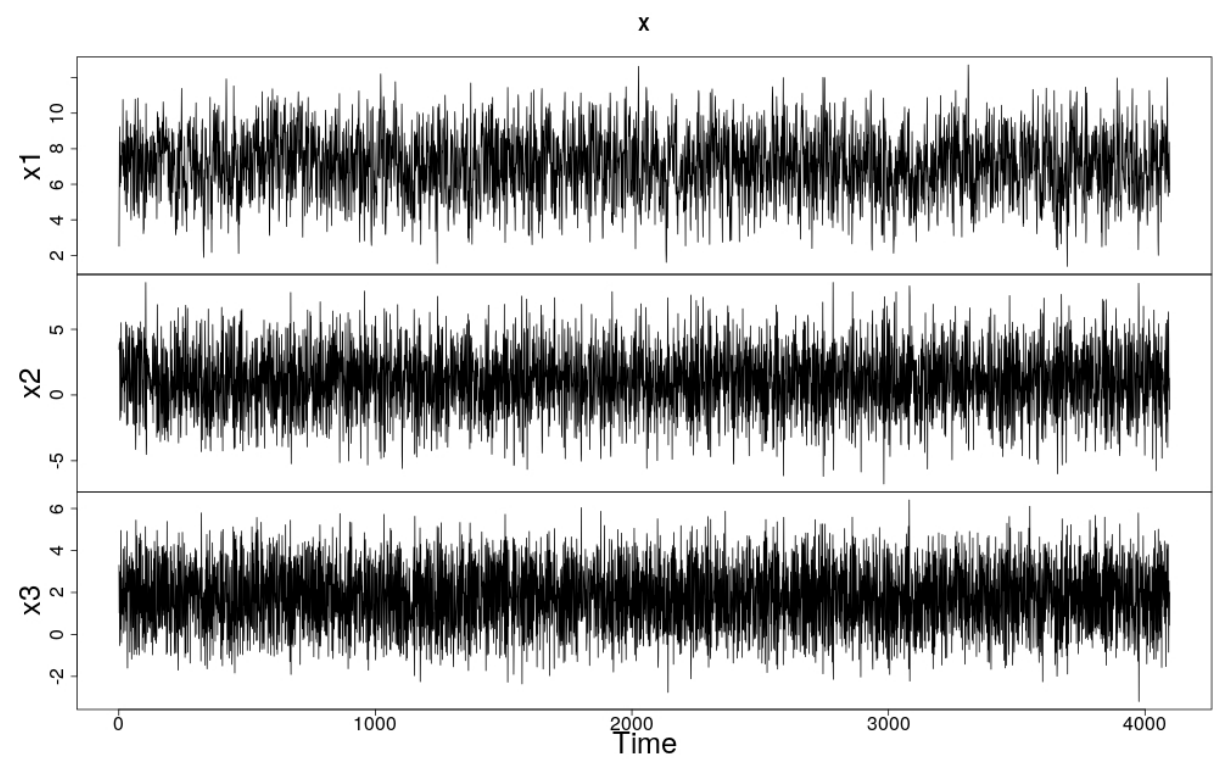

Figure 2.2. Example of simulated $\operatorname{VAR}(1)$.

analogously. The value $M$ defines how local the analysis will be: If $M$ is small, local and short-term influences will be estimated and the global behavior might be misinterpreted. If $M$ is too large we might not have stationarity.

The locally stationarity assumption allows us to evaluate the PDC along the time using a moving window. For instance, we can consider a variation of the Example 2.2 as follows.

Example 2.3. Consider the segmented VAR(1) process:

$$
\left[\begin{array}{l}
x_{1, t} \\
x_{2, t} \\
x_{3, t}
\end{array}\right]=\mathbf{A} \cdot\left[\begin{array}{l}
x_{1, t-1} \\
x_{2, t-1} \\
x_{3, t-1}
\end{array}\right]+\left[\begin{array}{l}
\varepsilon_{1, t} \\
\varepsilon_{2, t} \\
\varepsilon_{3, t}
\end{array}\right],
$$

with $\varepsilon_{i, t} \sim W N(0,1)$ and

$$
\begin{aligned}
\mathbf{A} & =\left[\begin{array}{rrr}
0.5 & 0.3 & 0.4 \\
-0.5 & 0.3 & 1 \\
0 & -0.3 & -0.2
\end{array}\right] . \mathbb{I}(t \leq 1365)+\left[\begin{array}{rrr}
0.5 & 0.3 & 0.4 \\
0 & 0.3 & 1 \\
0 & 0 & -0.2
\end{array}\right] . \mathbb{I}(1365<t \leq 2730)+ \\
& +\left[\begin{array}{rrr}
0.5 & 0 & 0 \\
-0.5 & 0.3 & 0 \\
1 & -0.3 & -0.2
\end{array}\right] . \mathbb{I}(2730<t \leq 4096) .
\end{aligned}
$$

In this example, we maintain the dependency structure of Example 2.2 in the first third of the sampled time and remove the forward dependencies $x_{1} \rightarrow x_{2}$ and $x_{2} \rightarrow x_{3}$ in the second third. For the last third, we remove all the backward relations $x_{2} \rightarrow x_{1}, x_{3} \rightarrow x_{1}$ and $x_{3} \rightarrow x_{2}$ and add the relation $x_{1} \rightarrow x_{3}$. The result of the time-varying PDC is presented in Figure 2.4. The black vertical bars indicate the time where the pattern changes happened. As we can see, calculating the PDC 

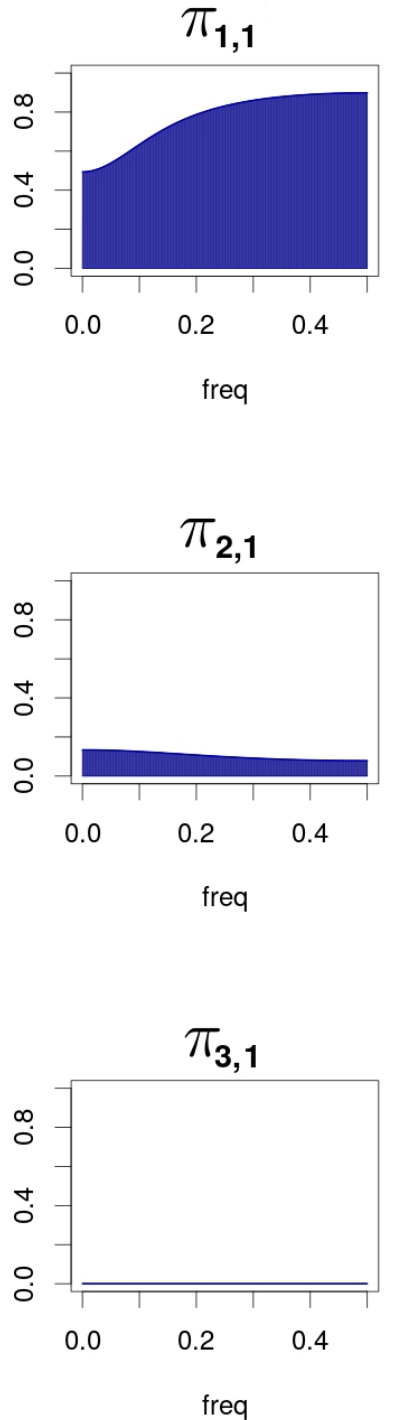
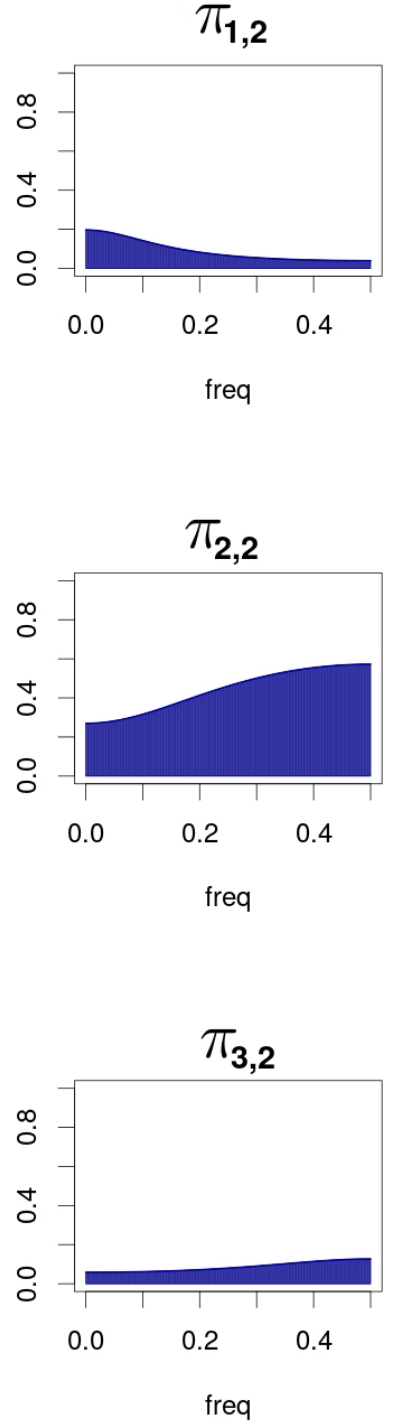
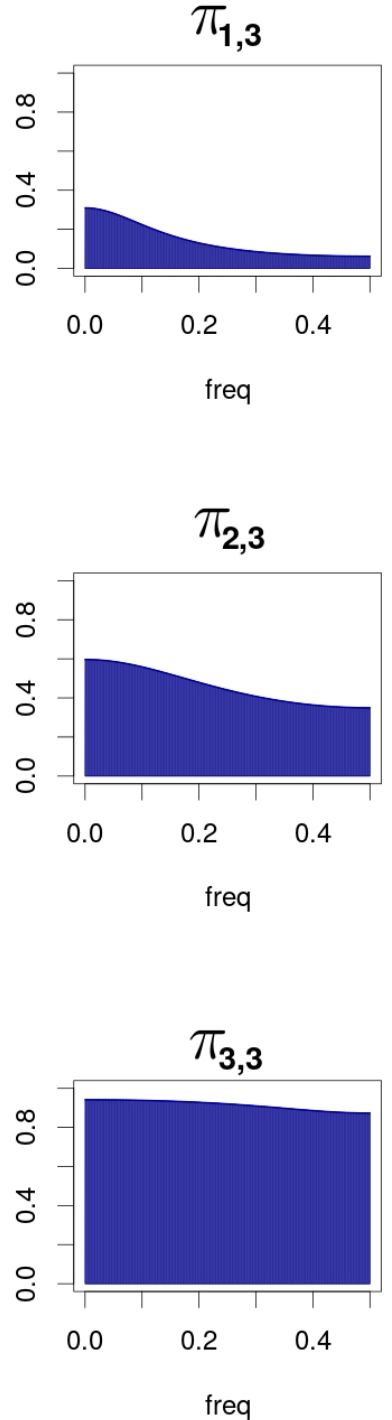

Figure 2.3. $P D C$ for the simulated $V A R(1)$ in Example 2.2. Each graph represents a directed relation between two signals, measured by the PDC. Notice that the magnitudes of the relations are proportional to the coefficients of the simulated model (2.20).

over the moving windows is a good alternative for locally stationary processes and can produce consistent results with the simulated data. Except for the graph "tv-PDC $(1,2)$ " and the last third of "tv-PDC $(3,2) "$, all the graphs presented the expected result.

\subsection{Locally Stationary Processes and Evolutionary Spectrum}

Stationary processes have good properties and allow the construction of a wide range of models. However, not all processes are stationary and it is not recommended to use the usual techniques of spectral representation for these processes, since it does not satisfy Wiener-Khintchine theorem's conditions. Nonetheless, there are alternatives to compute the spectrum, but the non-stationary process must satisfy some properties. We may cite other works like Martin and Flandrin (1985), that 

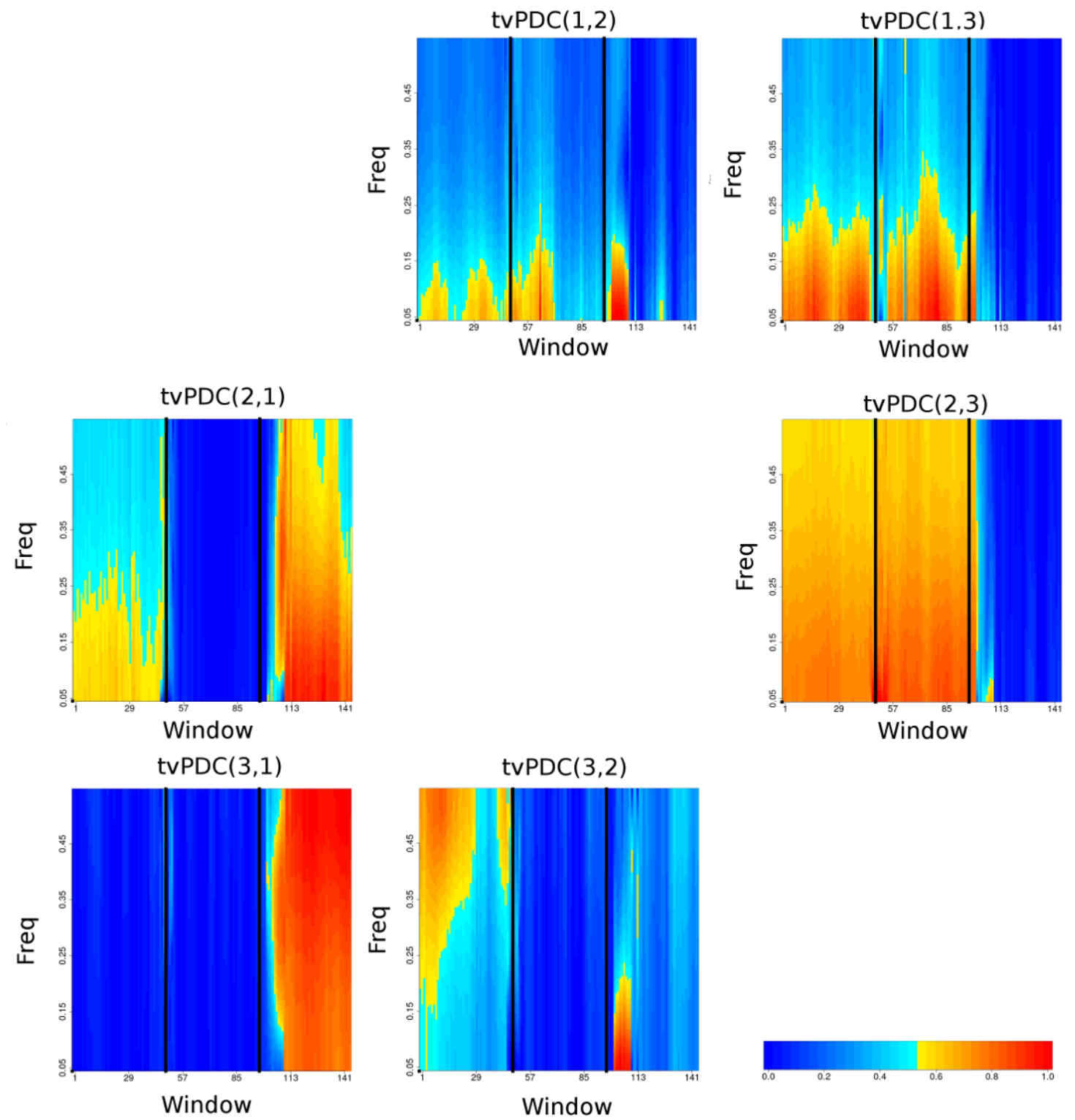

Figure 2.4. time-varying PDC for the simulated VAR(1) of Example 2.3.

proposed a Wigner-Ville Spectral Analysis. They have defined a class of Harmonizable Processes and the Conjoint Time-Frequency Representation by using the Wigner-Ville spectrum as an alternative to non-stationary bidimensional Fourier-Stieltjes transform of the autocovariance function. The Generalized Evolutionary Spectral Analysis proposed by Matz et al. (1997) introduces a family of timevarying spectra that contains Priestley's Evolutionary Spectra and two other spectra, the Transitory Evolutionary Spectrum and Weyl Spectrum. We present in this text two approaches: the Evolutionary Spectral Theory from Priestley (1965) and Dahlhaus' Evolutionary Spectrum for Locally Stationary Processes (Dahlhaus (1996)). Further details on Spectral Analysis for Nonstationary Processes can be found in Morettin (2014), Priestley (1981), Tong (1990) or in Subba Rao and Gabr (1984).

To deal with non-stationarity, Priestley (1965) proposed the Evolutionary Spectral Theory. The author expanded Fourier Spectral Analysis for non-stationary zero-mean processes $\left\{X_{t}\right\}$ with $E\left(X_{t}^{2}\right)<\infty$ 
that admit the following representation:

$$
X_{t}=\int_{-\infty}^{\infty} \phi_{t}(\omega) d Z(\omega)
$$

with $Z(\omega)$ an orthonormal process with $E|d Z(\omega)|^{2}=d \mu(\omega)$. In other words, $d \mu(\omega)$ is analogous to the spectrum in the stationary case. If the family of functions $\mathcal{F}=\left\{\phi_{t}(\omega)\right\}$ exists - as well as the measure $\mu(\omega)$ that allows this representation - then $\left\{X_{t}\right\}$ is called oscillatory process and the $\mathcal{F}$ family is named semi-stationary. Priestley and Tong (1973) proposed Evolutionary Cross Spectra and Coherency function based on this quantity to study relations between components in multivariate processes. Under these conditions, $X_{t}$ 's covariance function $\gamma(s, t)$ admits the following representation (Priestley (1981)):

$$
\gamma(s, t)=\int_{-\infty}^{\infty} \phi_{t}^{*}(\omega) \phi_{t}(\omega) d \mu(\omega)
$$

By restricting $\phi_{t}(\omega)=A_{t}(\omega) e^{i \theta t}$, i.e., considering

$$
X_{t}=\int_{-\infty}^{\infty} A_{t}(\omega) e^{i \theta t} d Z(\omega)
$$

as a particular case of (2.22), then from (2.23) we have

$$
\gamma(s, t)=\int_{-\infty}^{\infty} A_{s}^{*}(\omega) A_{t}^{*}(\omega) e^{i \omega(t-s)} d \mu(\omega)
$$

where $A_{t}(\omega)$ is of the form

$$
A_{t}(\omega)=\int_{-\infty}^{\infty} e^{i t \theta} d K_{\omega}(\theta)
$$

with $\left|d K_{\omega}(\theta)\right|$ having absolute maximum at $\theta=0$. Now we can present the Evolutionary (power) Spectrum as described by Priestley (1981).

Definition 2.4 (Priestley (1981)). Let $\mathcal{F}$ denote a particular family of oscillatory functions, $\left\{\phi_{t}(\omega) \equiv\right.$ $\left.A_{t}(\omega) e^{i \omega t}\right\}$, and let $X_{t}$ be an oscillatory process having a representation of the form (2.23) in terms of the family $\mathcal{F}$. Then

$$
d H_{t}(\omega)=\left|A_{t}(\omega)\right|^{2} d \mu(\omega)
$$

is called evolutionary power spectrum at time $t$ with respect to the family $\mathcal{F}$.

When $X_{t}$ is stationary and the chosen family $\mathcal{F}$ is the family of complex exponentials, then (2.24) coincides with the usual definition of spectrum. Priestley (1996) have discussed the properties of the evolutionary spectrum and the interpretation of its relation with the wavelet transform.

Other important approach to deal with these processes is to consider Dahlhaus' concept of locally stationarity (Dahlhaus (1996)), in which increasing the sample size means bringing more information to the local structures. It is defined it as follows: 
Definition 2.5. A sequence of stochastic processes $X_{t, T}$ is locally stationary with transfer function $A^{0}$ and trend $\mu$ if there exists a representation

$$
X_{t, T}=\mu\left(\frac{t}{T}\right)+\int_{-\pi}^{\pi} \exp (i \omega t) A_{t, T}^{0}(\omega) d \zeta(\omega)
$$

where

- $\zeta$ is a stochastic process on $[\pi, \pi]$, with $\zeta^{*}(\omega)=\zeta(-\omega)$ and

$$
\operatorname{cum}\left\{d \zeta\left(\omega_{1}\right), \ldots, d \zeta\left(\omega_{k}\right)\right\}=\eta\left(\sum_{j=1}^{k} \omega_{j}\right) g_{k}\left(\omega_{1}, \ldots, \omega_{k-1}\right) d \omega_{1} \ldots d \omega_{k}
$$

where $\operatorname{cum}\{\ldots\}$ denotes the cumulant of $k$-th order, $g_{1}=0, g_{2}(\lambda)=1,\left|g_{k}\left(\omega_{1}, \ldots, \omega_{k-1}\right)\right| \leq$ const $_{k}, \forall k$ and $\eta(\omega)=\sum_{j=-\infty}^{\infty} \delta(\omega+2 \pi j)$ is the period $2 \pi$ extension of the Dirac delta function.

- There exists a constant $K$ and a $2 \pi$-periodic function $A:[0,1] \times \mathbb{R} \rightarrow \mathbb{C}$ with $A(u,-\lambda)=$ $A^{*}(u, \lambda)$ and

$$
\sup _{t, \lambda}\left|A_{t, T}^{0}(\omega)-A(t / T, \omega)\right| \leq K T^{-1}
$$

$\forall T . A(u, \omega)$ and $\mu(u)$ are assumed to be continuous in $u$.

In that definition $A(u, \omega)$ and $\mu(u)$ are assumed to be continuous in $u$. In Dahlhaus and Polonik (2006), the authors present an analogous definition that does not require continuity in the parameter curves but only bounded variation. To introduce this definition, consider:

$$
V(g)=\sup \left\{\sum_{k=1}^{m}\left|g\left(x_{k}\right)-g\left(x_{k-1}\right)\right| 0 \leq x_{0}<\ldots<x_{m} \leq 1, m \in \mathbb{N}\right\}
$$

the total variation of a function $g$ on $[0,1]$. Also, for some $k>0$ and $j \in \mathbb{Z}$, let

$$
l(j)= \begin{cases}1, & |j| \leq 1 \\ |j|(\log |j|)^{1+k}, & |j|>1 .\end{cases}
$$

Definition 2.6 (Locally stationary processes - Dahlhaus and Polonik (2006)). The sequence $X_{t, T}, t=$ $1, \ldots, T$ is a locally stationary process if it has the representation

$$
X_{t, T}=\sum_{j=-\infty}^{\infty} a_{t, T}(j) \varepsilon_{t-j}
$$

where the $\varepsilon_{t}$ are identically distributed with $\mathbb{E}\left(\varepsilon_{t}\right) \equiv 0, \mathbb{E}\left(\varepsilon_{s} \varepsilon_{t}\right) \equiv 0$ for $s \neq t, \mathbb{E}\left(\varepsilon_{t}^{2}\right) \equiv 1$,

$$
\sup _{t}\left|a_{t, T}(j)\right| \leq \frac{K}{l(j)} \text { (with } K \text { not depending on } T \text { ) }
$$


and there exists functions $a(., j):(0,1] \rightarrow \mathbb{R}$ with

$$
\begin{aligned}
\sup _{u}|a(u, j)| & \leq \frac{K}{l(j)}, \sup _{j} \sum_{t=1}^{T}\left|a_{t, T}(j)-a\left(\frac{t}{T}, j\right)\right| \leq \frac{K}{l(j)} \\
V(a(., j)) & \leq \frac{K}{l(j)} .
\end{aligned}
$$

Dahlhaus and Polonik (2009) have shown that time-varying ARMA (tv-ARMA) with bounded variation coefficients fulfills the above conditions.

This approach is important to develop an asymptotic theory of locally stationary processes. For instance, using the Definition 2.6, Nason et al. (2000) defined and formalized the locally stationary wavelet processes and the evolutionary wavelet spectrum, as well as the estimation theory and asymptotic results for the wavelet periodogram and wavelet smoothed periodogram.

For this definition, the Evolutionary Spectrum is given by

$$
f(u, \omega)=|A(u, \omega)|^{2} .
$$

Neumann and von Sachs (1997) show that if

$$
f_{T}(u, \omega)=\frac{1}{2 \pi} \sum_{s} \operatorname{Cov}\left(X_{(u T-s / 2), T}, X_{(u T+s / 2), T}\right) e^{-i \omega s}
$$

then

$$
f_{T}(u, \omega) \underset{T \rightarrow \infty}{\stackrel{m s}{\longrightarrow}} f(u, \omega) .
$$

\subsection{Wavelets}

Fourier analysis is appropriate to analyze stationary processes, while wavelet analysis is appropriate for situations with abrupt pattern changes, outliers and processes with local characteristics. Its local indexation and smoothness adaptability along scales can evaluate long and short-term variations, and local patterns.

A wavelet is a function $\psi(t)$ that satisfies the following mathematical conditions (Morettin (2014)):

(i) $\int_{-\infty}^{\infty} \psi(t) d t=0$;

(ii) $\int_{-\infty}^{\infty}|\psi(t)| d t<\infty$;

(iii) $\frac{|\Psi(\omega)|^{2}}{|\omega|} d \omega<\infty$, where $\Psi(\omega)$ is the Fourier transform of $\psi(t)$;

(iv) $\int_{-\infty}^{\infty}|\psi(t)|^{2} d t=1$ or $\int_{-\infty}^{\infty}|\Psi(\omega)| d t=2 \pi$;

(v) $\int_{-\infty}^{\infty} t^{j} \psi(t) d t=0, j=0,1,2, \ldots, r-1$, for at least a $r \geq 1$ and $\int_{-\infty}^{\infty}\left|t^{r} \psi(t)\right| d t<\infty$. 
For example, the Haar wavelet is the most simple wavelet, given by:

$$
\psi(t)= \begin{cases}-1, & -1 / 2 \leq t \leq 0 \\ 1, & 0 \leq t \leq 1 / 2 \\ 0, & \text { otherwise }\end{cases}
$$

Other examples of wavelets are the Mexican hat wavelet

$$
\psi(t)=\left(1-t^{2}\right) e^{-t^{2} / 2}
$$

and the Morlet wavelet

$$
\psi(t)=e^{i w_{0} t} e^{-t^{2} / 2} \text {, for some } w_{0} \text { fixed. }
$$

Another important wavelets are the Daubechies wavelets (Daubechies (1988) and Daubechies (1992)). These wavelets are orthogonal, asymmetric and have compact support.

Therefore, a wavelet is a small wave and, differently of a sine function that periodically oscillates all over the real axis, it has its energy concentrated around one region and decaying to zero in the rest of the axis. Figure 2.5 (a) presents the sine function and figures 2.5 (b)-(f) are examples of wavelets.

By dilating and translating $\psi(t)$ (the mother wavelet) we generate the space of functions
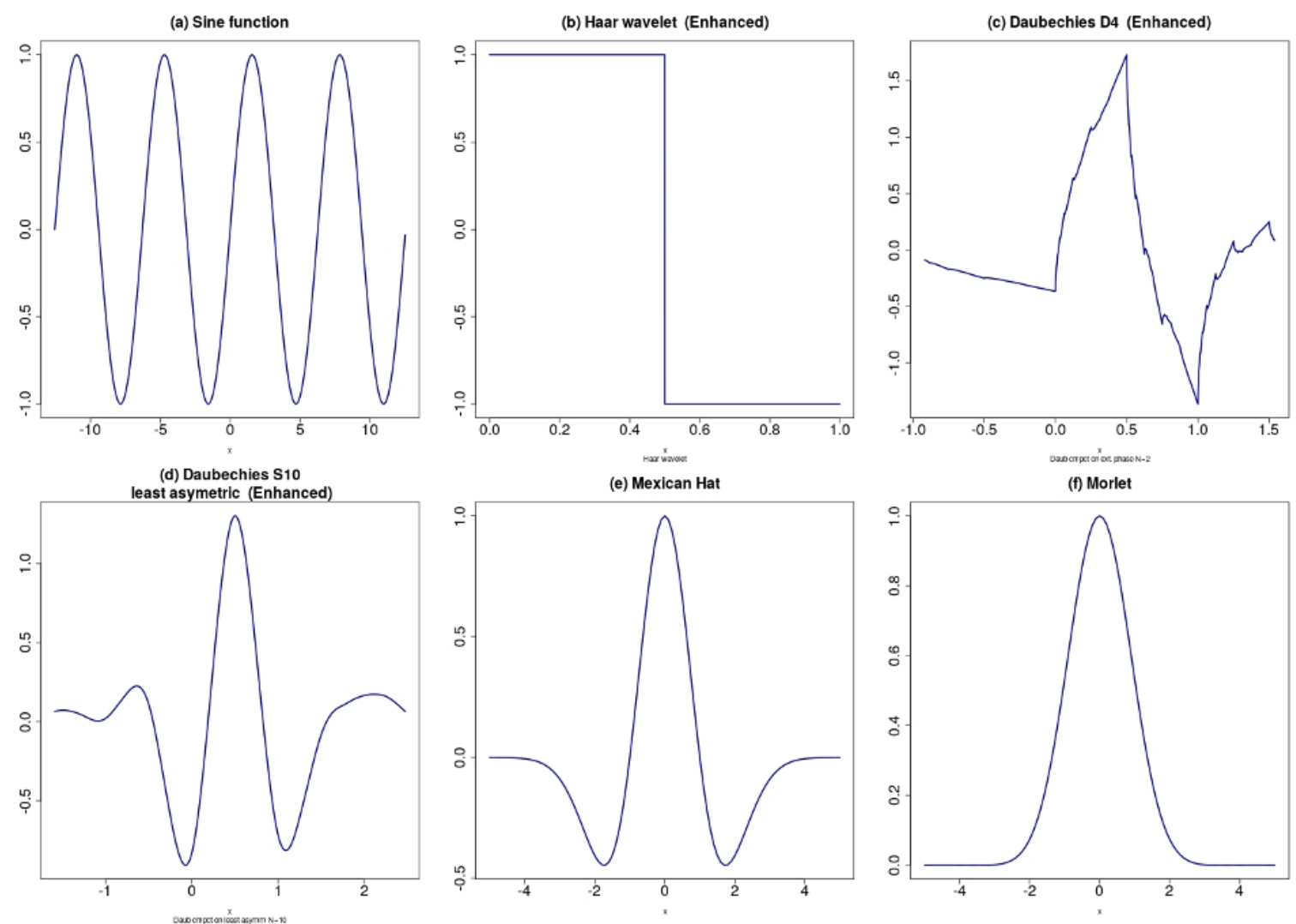

Figure 2.5. (a) sine function: it oscillates all over the real axis. (b) the Haar wavelet, (c) Daubechies D4 wavelet, (d) the Daubechies S10 least asymmetric, (e) Mexican hat wavelet and (f) real part of the Morlet wavelet. 


$$
\left\{\psi_{j, k} \mid \psi_{j, k}=2^{j / 2} \psi\left(2^{j} t-k\right), j, k \in \mathbb{Z}\right\}
$$

On the other hand the function (the father wavelet, or scaling function)

$$
\phi(t)=\sqrt{2} \sum_{k} l_{k} \phi(2 t-k)
$$

generates an orthonormal family of $L^{2}(\mathbb{R})$,

$$
\phi_{j, k}(t)=2^{j / 2} \phi\left(2^{j} t-k\right), j, k \in \mathbb{Z},
$$

such that we can obtain $\psi(t)$ from

$$
\psi(t)=\sqrt{2} \sum_{k} h_{k} \phi(2 t-k)
$$

where

$$
h_{k}=(-1)^{k} g_{1-k} .
$$

$g_{k}$ and $h_{k}$ are low-pass and high-pass filters respectively and are given by:

$$
\begin{aligned}
& g_{k}=\sqrt{2} \int_{-\infty}^{\infty} \phi(t) \phi(2 t-k) d t \\
& h_{k}=\sqrt{2} \int_{-\infty}^{\infty} \psi(t) \phi(2 t-k) d t .
\end{aligned}
$$

Now, for $f(t) \in L^{2}(\mathbb{R})$ we can represent it as

$$
f(t)=\sum_{k} c_{j_{0}, k} \phi_{j_{0}, k}(t)+\sum_{j \geq j_{0}} \sum_{k} d_{j, k} \psi_{j, k}(t)
$$

where

$$
\begin{aligned}
c_{j_{0}, k} & =\int_{-\infty}^{\infty} f(t) \phi_{j_{0}, k} d t \\
d_{j, k} & =\int_{-\infty}^{\infty} f(t) \psi_{j, k} d t
\end{aligned}
$$

are the scaling and wavelet coefficients, respectively. They represent smooth, long-term variations and detail, short-term variations (differences) of the function $f(t)$, respectively. It is usual to consider $2^{j_{0}-1}=0$ and, as Sato et al. (2007) describe, the coefficients $d_{j, k}$ are associated with changes of averages on scale $2^{j-1}$ units apart, and $c_{j_{0}, k}$ are smoothed averages over scale $j_{0}$. Increasing one unit in $j$ will lead to an increase of $2^{j+1}-2^{j}$ on the number of resolution points. The coefficient $k$ is the time location coefficient, indicating translations of the wavelet functions.

So, we can write any function $f(t) \in L^{2}(\mathbb{R})$ as a decomposition of scaling and wavelet coefficients. $c_{j_{0}, k}$ and $d_{j, k}$ are the representation of the function $f(t) \in L^{2}(\mathbb{R})$ in the wavelet domain and they represent how local weighted averages of $f($.) vary from one period of time to another. They also can 
tell us if these variations in $f($.$) are happening in small, short-term scale or in a persistent, long-term$ scale, depending the width of the filters composing $c_{j_{0}, k}$ and $d_{j, k}$.

\subsubsection{Multiresolution Analysis}

Let $V_{j}$ be the space generated by the orthonormal basis $\left\{\phi_{j k}\right\}$ in (2.29). We can show that the sequence $\ldots, V_{-1}, V_{0}, V_{1}, \ldots$ is a crescent sequence of nested spaces, i.e.

$$
\cdots \subset V_{-1} \subset V_{0} \subset V_{1} \subset \ldots
$$

and $\cup_{j} V_{j}$ is dense in $L^{2}(\mathbb{R})$. Now, there is a $W_{j}$ orthonormal complement of $V_{j}$ such that

$$
V_{j+1}=V_{j} \oplus W_{j}
$$

and this complement is generated from the basis $\left\{\psi_{j k}\right\}$ in equation (2.28) i.e., $\left\{\psi_{j k}\right\}$ is an orthonormal basis in $W_{j}$. Figure 2.6 illustrates this situation for $v_{j}, w_{j}$ and $v_{j+1}$ any elements of $V_{j}, W_{j}$ and $V_{j+1}$, respectively.

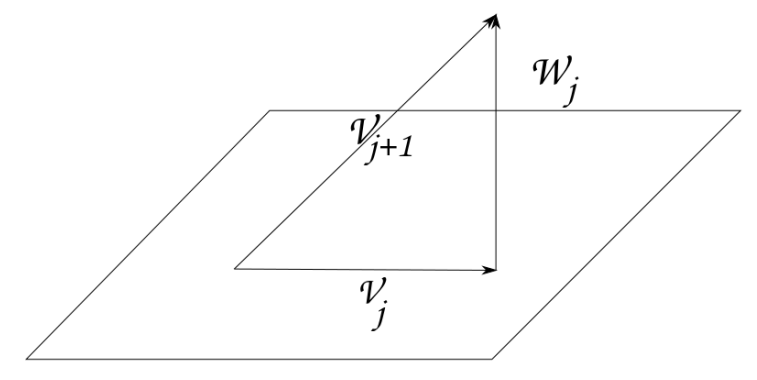

Figure 2.6. Illustration of orthonormal complement $W_{0}$

From (2.33) we can recursively write:

$$
V_{j+1}=V_{j} \oplus W_{j}=V_{j-1} \oplus W_{j-1} \oplus W_{j}=\cdots=V_{0} \oplus W_{0} \oplus W_{1} \oplus \cdots \oplus W_{j}=V_{0} \bigoplus_{l=0}^{j} W_{l} .
$$

Finally, since $\cup_{j} V_{j}$ is dense in $L^{2}(\mathbb{R})$,

$$
L^{2}(\mathbb{R})=V_{0} \bigoplus_{j=0}^{\infty} W_{j}
$$

i.e., we can represent any element in $L^{2}(\mathbb{R})$ by using a combination of elements from $V_{0}$ and $W_{j}$ 's, as represented in equation (2.32). From Härdle et al. (1997) we have that

$$
\left\{\left\{\phi_{0 k}\right\},\left\{\psi_{j k}\right\}, k \in \mathbb{Z}, j=0,1,2, \ldots\right\}
$$


is an orthonormal basis in $L^{2}(\mathbb{R})$.

So, instead of having one resolution level (from sines and cosines basis) as in Fourier analysis, wavelet analysis has several resolution levels. Formally, Härdle et al. (1997) define a multiresolution analysis as

Definition 2.7. Let $\left\{\phi_{0 k}\right\}$ be an orthonormal system in $L^{2}(\mathbb{R})$. The sequence of spaces $\left\{V_{j}, j \in \mathbb{Z}\right\}$, generated by $\phi$ is called a multiresolution analysis $(M R A)$ of $L^{2}(\mathbb{R})$ if $V_{j} \subset V_{j+1}, j \in \mathbb{Z}$, and $\cup_{j \geq 0} V_{j}$ is dense in $L^{2}(\mathbb{R})$.

The multiresolution analysis is mainly what makes wavelet analysis more effective than Fourier analysis. It allows us to evaluate local properties and better represent functions like function (1.1) presented on Example 1.1. Notice that is not necessary that the function to be periodic or continuous. It can have a finite number of discontinuities as long as is a function of $L^{2}(\mathbb{R})$.

\subsubsection{Continuous Wavelet Transform}

In this thesis we will focus on the Discrete Wavelet Transforms. Thus, in this section we will briefly define the Continuous Wavelet Transform (CWT) but we will not go in details of its properties. This section is mainly based in Morettin (2014). Let $f(t)$ be a function in $L^{2}(\mathbb{R})$ and $\psi(t)$ a wavelet that satisfies the conditions i-v at the beginning of the section. Then, the Continuous Wavelet Transform (CWT) of $f(t)$ is

$$
W_{\psi} f(a, b)=|a|^{1 / 2} \int_{-\infty}^{\infty} f(t) \psi\left(\frac{t-b}{a}\right) d t, a>0, b \in \mathbb{R},
$$

Notice that we are using a one dimensional, continuous signal $f(t)$ to create a two-dimensional continuous signal, $\psi(a>0, b \in \mathbb{R})$, which causes a lot of redundancy in CWT. When we use the Discrete Wavelet Transform (DWT) we mitigate this redundancy by subsampling the scales in a way that still preserves important features of $f(t)$ represented in the wavelet domain.

\subsubsection{Discrete Wavelet Transform}

The Discrete Wavelet Transform is a mathematical operation that creates a representation of an object from real domain, say $\mathbf{X}^{\top}=\left[X_{0, T}, \ldots, X_{T-1, T}\right]$, in the wavelet domain. If we consider it as an orthonormal transformation and let $\left\{W_{j, t}: j=0, \ldots, J, t=0, \ldots, L_{j}\right\}$ be the DWT coefficients then we have

$$
\mathbf{W}=\mathcal{W} \mathbf{X}
$$

where $\mathbf{W}=\left\{W_{j, t}\right\}$ is a $T \times 1$ vector and is the representation of $\mathbf{X}$ in the wavelet domain. We also restrict $T=2^{J}, J \in \mathbb{N}$. Since the DWT is an orthonormal transformation, we have $\mathcal{W} \times \mathcal{W}=I_{T}$ and $\mathcal{W}$ represent the wavelet filters that transform $\mathbf{X}$ from real domain to the wavelet domain. Before explicit the form of $\mathbf{W}$ and $\mathcal{W}$ we present the low-pass and high-pass filters $\left\{g_{j, l}\right\}$ and $\left\{h_{j, l}\right\}$. Let $X_{t, T}$ be a discrete time, real valued process and $\left\{h_{j, l}: j=1, \ldots, J, l=0, \ldots, L_{j}-1\right\}$ a wavelet filter, with width $L_{j}=\left(2^{j}-1\right)(L-1)+1 . L=L_{1}$ (integer, even) and $J=\log _{2} T$. The wavelet 
filter $\left\{h_{j, l}\right\}$ satisfies the properties:

$$
\sum_{l=0}^{L_{j}-1} h_{j, l}=0 ; \quad \sum_{l=0}^{L_{j}-1} h_{j, l}^{2}=1 ; \quad \sum_{l=0}^{L_{j}-1} h_{j, l} h_{j, l+2^{j} n}=\sum_{l=-\infty}^{\infty} h_{j, l} h_{j, l+2^{j} n}=0,
$$

for all nonzero integers $n, h_{j, 0} \neq 0$ and $h_{j, L_{j}-1} \neq 0$. The first two properties are related to the orthonormality property of the wavelet filter.

The scaling filter, on the other hand, is a low-pass filter $\left\{g_{j, l}: j=1, \ldots, J, l=0, \ldots, L_{j}-1\right\}$ such that

$$
\sum_{l=0}^{L_{j}-1} g_{j, l}=2^{j / 2} ; \quad \sum_{l=0}^{L_{j}-1} g_{j, l}^{2}=1 ; \quad \sum_{l=0}^{L_{j}-1} g_{j, l} g_{j, l+2^{j} n}=\sum_{l=-\infty}^{\infty} g_{j, l} g_{j, l+2^{j} n}=0, n \neq 0
$$

for all nonzero integers $n, g_{j, 0} \neq 0$ and $g_{j, L-1} \neq 0$.

The coefficients associated with the $j$-th level are defined in Percival and Walden (2000) as follows:

$$
\begin{aligned}
2^{1 / 2} \widetilde{W}_{j, t} & \equiv \sum_{l=0}^{L_{j}-1} h_{j, l} X_{t-l \operatorname{Mod} T}, t=0, \ldots, T-1, \\
W_{j, t} & \equiv 2^{j / 2} \widetilde{W}_{j, 2^{j}(t+1)-1}=\sum_{l=0}^{L_{j}-1} h_{j, l} X_{2^{j}(t+1)-1-l \operatorname{Mod} T},
\end{aligned}
$$

with $t=0, \ldots, T_{j}-1, T_{j}=T / 2^{j}$ and $j \operatorname{Mod} T$ is the modulo operator and is defined as

$j \operatorname{Mod} T= \begin{cases}j & , 0 \leq j \leq T-1 \\ j-n T & , j \geq T \text { and } n \text { is the largest integer such that } 0 \leq j-n T \leq T-1 .\end{cases}$

When $j \operatorname{Mod} T<0, X_{j \operatorname{Mod} T}$ is periodically extended, e.g. $X_{-1}=X_{T-1} ; X_{-2}=X_{T-2}$ and so forth. $W_{j, t}$ is the wavelet representation of $X_{t, T}$ in scale $2^{j-1}$ and is called Discrete Wavelet Transform (DWT). For $j$ small we have short-term detailed variations while for $j$ next to $J$ we have smooth long-term variations of $X_{t, T}$.

Analogously, the low-pass filters $\left\{g_{j, l}\right\}$ generate the scaling coefficients by:

$$
\begin{aligned}
2^{1 / 2} \widetilde{V}_{j, t} & \equiv \sum_{l=0}^{L_{j}-1} g_{j, l} X_{t-l \operatorname{Mod} T}, t=0, \ldots, T-1 \\
V_{j, t} & \equiv 2^{j / 2} \widetilde{V}_{j, 2^{j}(t+1)-1}=\sum_{l=0}^{L_{j}-1} g_{j, l} X_{2^{j}(t+1)-1-l \operatorname{Mod} T},
\end{aligned}
$$

The filters $h_{j, l}$ and $g_{j, l}$ are calculated according to the filters $g_{j-1, l}$ as follows:

$$
h_{j, l}=\sum_{k=0}^{L_{j}-1} h_{k} g_{j-1, l-2^{j-1} k}
$$




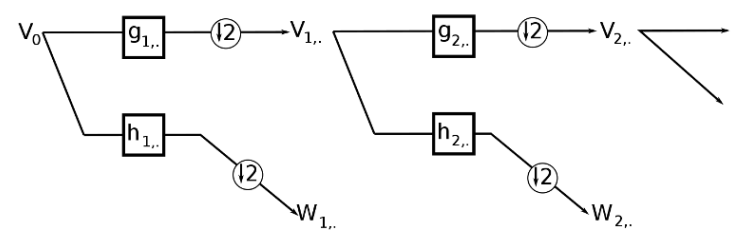

Figure 2.7. Mallat's algorithm (adapted). The symbol $\downarrow 2$ represents the decimation operation.

$$
g_{j, l}=\sum_{k=0}^{L_{j}-1} g_{k} g_{j-1, l-2^{j-1} k} .
$$

In Haar wavelet, for example, the $j$-th level scaling filter will be

$$
g_{j, l}= \begin{cases}(1 / 2)^{j / 2}, & l=0, \ldots, 2^{j}-1 \\ 0, & \text { otherwise. }\end{cases}
$$

From equations (2.40) and (2.41) is possible to write $W_{j, t}$ and $V_{j, t}$ as

$$
\begin{aligned}
W_{j, t} & =\sum_{l=0}^{L-1} h_{l} V_{j-1,2 t+1-l \operatorname{Mod} T_{j-1}}, \\
V_{j, t} & =\sum_{l=0}^{L-1} g_{l} V_{j-1,2 t+1-l \operatorname{Mod} T_{j-1}},
\end{aligned}
$$

i.e., $W_{j, t}$ and $V_{j, t}$ are resultant of successive low-pass and high-pass filtering operations of the previous scale's scaling coefficients.

The origin of these definition is Mallat's algorithm, that recursively applies the filters to create the scaling and wavelets coefficients. Figure 2.7, extracted from Morettin (2014), illustrates the process. The symbol ' $\downarrow 2$ ' represents the decimation operation, characterized by the sample reduction occurred in the scaling and wavelet coefficients definition in (2.42) and (2.43). In other words, each coefficient of level $j$, say $w_{j, t}$ (or $v_{j, t}$ for the scaling coefficients), is calculated using only half of the coefficients from the previous level, $v_{j-1,2 t+1-l}, l \in 1,2, \ldots, L_{j-1}$.

From Section 2.4.1 we know that

$$
L^{2}(\mathbb{R})=V_{J_{0}} \bigoplus_{j=J_{0}}^{\infty} W_{j}
$$

So it is clear that a representation of $\mathbf{X}$ in terms of $V_{J_{0}}$ and $W_{j}$ have the form:

$$
\mathbf{X}=\mathcal{W}^{\top} \mathbf{W}=\sum_{j=1}^{J_{0}} \mathcal{W}_{j}^{\top} \mathbf{W}_{j}+\mathcal{V}_{J_{0}}^{\top} \mathbf{V}_{\mathbf{J}_{\mathbf{0}}}=\sum_{j=1}^{J_{0}} \mathcal{D}_{j}+\mathcal{S}_{J_{0}}
$$

that is a multiresolution analysis (MRA) of $\mathbf{X} . \mathbf{V}_{\mathbf{J}_{\mathbf{0}}}$ is an $T_{J_{0}}$ dimensional vector of scaling coefficients associated with averages on scale $\tau_{J_{0}}=2^{J_{0}}$ and $\mathcal{V}_{J_{0}}$ is an $T_{J_{0}} \times T$ dimensional matrix of the 
scaling filters. $\mathbf{W}_{j}$ is an $T_{j}=T / 2^{j}$ dimensional vector of wavelet coefficients associated with scale $\tau_{j}=2^{j-1}$ and $\mathcal{W}_{j}$ is an $T \times T$ dimensional matrix. In equation (2.35) we must then have:

$\mathbf{W}^{\top}=\left[\begin{array}{lllll}\mathbf{V}_{J_{0}} & \mathbf{W}_{1} & \mathbf{W}_{2} & \ldots & \mathbf{W}_{J_{0}}\end{array}\right] \quad$ and $\quad \mathcal{W}^{\top}=\left[\begin{array}{llllll}\mathcal{V}_{J_{0}} & \mathcal{W}_{1} & \mathcal{W}_{2} & \ldots & \mathcal{W}_{J_{0}}\end{array}\right]$.

The first row of $\mathcal{W}$, row $\mathcal{V}_{J_{0}}$, represents the low-pass filter $g_{J_{0}, l}$. The next rows are the high-pass filters $h_{j, l}$. For each scale $\tau_{j}$ we will have $L_{j}$ rows, each one corresponding to one wavelet coefficient. For example, if we have $T=8$ and we use the Haar wavelet $(L=2)$, the next four rows are related to the scale $\tau_{J}=\tau_{3}=2^{J-1}=4\left(\mathcal{W}_{1, \cdot,}, \ldots, \mathcal{W}_{4,}\right)$ followed by two rows of scale $\tau_{2}=2\left(\mathcal{W}_{5, \cdot,}, \mathcal{W}_{6,}\right)$ and one row of scale $\tau_{1}=1\left(\mathcal{W}_{7,}\right)$.

Notice that for each scale $\tau_{j}$, its rows are an orthonormal basis for space $W_{j}$ of the multiresolution analysis and therefore, must be orthogonal. Consider then the linear shifting operator $\mathcal{T}^{k}$, that given a vector $X=\left[x_{1}, \ldots, x_{N}\right]$, shifts its components in $k$ positions, i.e.,

$$
\begin{aligned}
\mathcal{T} X & =\left[x_{N}, x_{1}, x_{2} \ldots, x_{N-1}\right], \\
\mathcal{T}^{2} X & =\left[x_{N-1}, x_{N}, x_{1}, x_{2}, \ldots, x_{N-2}\right],
\end{aligned}
$$

and so forth. Thus, the first row of each scale starts with the $L_{j}$ coefficients and the rest of the vector is filled with zeros The next vector of the scale is the first vector shifted $L_{j}$ positions, the second next is shifted $L L_{J}$ positions and so forth until we complete all vectors of the scale $\tau_{j}$. In the example with $T=8$ and Haar wavelet $(L=2)$, we have then:

$$
\begin{aligned}
& \mathcal{W}_{2, \bullet}=\mathcal{T}^{2} \mathcal{W}_{1, \cdot} ; \quad \mathcal{W}_{3, \bullet}=\mathcal{T}^{4} \mathcal{W}_{1, \cdot} ; \quad \mathcal{W}_{4, \bullet}=\mathcal{T}^{6} \mathcal{W}_{1, \bullet} ; \\
& \mathcal{W}_{6, \bullet}=\mathcal{T}^{4} \mathcal{W}_{5, ;}
\end{aligned}
$$

To illustrate how the DWT is calculated, consider this example extracted from Morettin (2014).

Example 2.4 (Morettin (2014)). Consider the vector $x=\{2,-1,0,3,1,2,-1,2\}$ and calculate its DWT. For $N=8$ we have $J=3$ and therefore, using Haar wavelet, the matrix $\mathcal{W}$ is given as follow:

$$
\mathcal{W}=\left(\begin{array}{cccccccc}
\frac{\sqrt{2}}{4} & \frac{\sqrt{2}}{4} & \frac{\sqrt{2}}{4} & \frac{\sqrt{2}}{4} & \frac{\sqrt{2}}{4} & \frac{\sqrt{2}}{4} & \frac{\sqrt{2}}{4} & \frac{\sqrt{2}}{4} \\
\frac{\sqrt{2}}{2} & -\frac{\sqrt{2}}{2} & 0 & 0 & 0 & 0 & 0 & 0 \\
0 & 0 & \frac{\sqrt{2}}{2} & -\frac{\sqrt{2}}{2} & 0 & 0 & 0 & 0 \\
0 & 0 & 0 & 0 & \frac{\sqrt{2}}{2} & -\frac{\sqrt{2}}{2} & 0 & 0 \\
0 & 0 & 0 & 0 & 0 & 0 & \frac{\sqrt{2}}{2} & -\frac{\sqrt{2}}{2} \\
\frac{1}{2} & \frac{1}{2} & -\frac{1}{2} & -\frac{1}{2} & 0 & 0 & 0 & 0 \\
0 & 0 & 0 & 0 & \frac{1}{2} & \frac{1}{2} & -\frac{1}{2} & -\frac{1}{2} \\
\frac{\sqrt{2}}{4} & \frac{\sqrt{2}}{4} & \frac{\sqrt{2}}{4} & \frac{\sqrt{2}}{4} & -\frac{\sqrt{2}}{4} & -\frac{\sqrt{2}}{4} & -\frac{\sqrt{2}}{4} & -\frac{\sqrt{2}}{4}
\end{array}\right)
$$

Calculating the product in (2.35), we have:

$$
\begin{aligned}
& \mathbf{W}=\left(\mathbf{V}_{\mathbf{3}}, \mathbf{W}_{\mathbf{1}}, \mathbf{W}_{\mathbf{2}}, \mathbf{W}_{\mathbf{3}}\right) \\
& \mathbf{W}=\left(V_{3,0}, W_{1,0}, W_{1,1}, W_{1,2}, W_{1,3}, W_{2,0}, W_{2,1}, W_{3,0}\right)
\end{aligned}
$$




$$
\begin{aligned}
V_{3,0} & =\frac{\sqrt{2}}{4}\left(x_{7}+x_{6}+x_{5}+x_{4}+x_{3}+x_{2}+x_{1}+x_{0}\right) ; \\
W_{1,0} & =\frac{\sqrt{2}}{2}\left(x_{1}-x_{0}\right) ; \quad W_{1,1}=\frac{\sqrt{2}}{2}\left(x_{3}-x_{2}\right) ; \quad W_{1,2}=\frac{\sqrt{2}}{2}\left(x_{5}-x_{4}\right) ; \quad W_{1,3}=\frac{\sqrt{2}}{2}\left(x_{7}-x_{6}\right) ; \\
W_{2,0} & =\frac{1}{2}\left[\left(x_{2}+x_{3}\right)-\left(x_{0}+x_{1}\right)\right] ; \quad W_{2,1}=\frac{1}{2}\left[\left(x_{6}+x_{7}\right)-\left(x_{4}+x_{5}\right)\right] ; \\
W_{3,0} & =\frac{\sqrt{2}}{4}\left[\left(x_{7}+x_{6}+x_{5}+x_{4}\right)-\left(x_{3}+x_{2}+x_{1}+x_{0}\right)\right] ;
\end{aligned}
$$

Notice that the wavelet coefficients (details, $W_{j, k}$ ) are related to differences between local averages while the scaling coefficients (smooth, $V_{j, k}$ ) are calculated as the average of the observations. Figure 2.8 illustrates the calculations for $W_{j, k}$ and $V_{j, k}$.

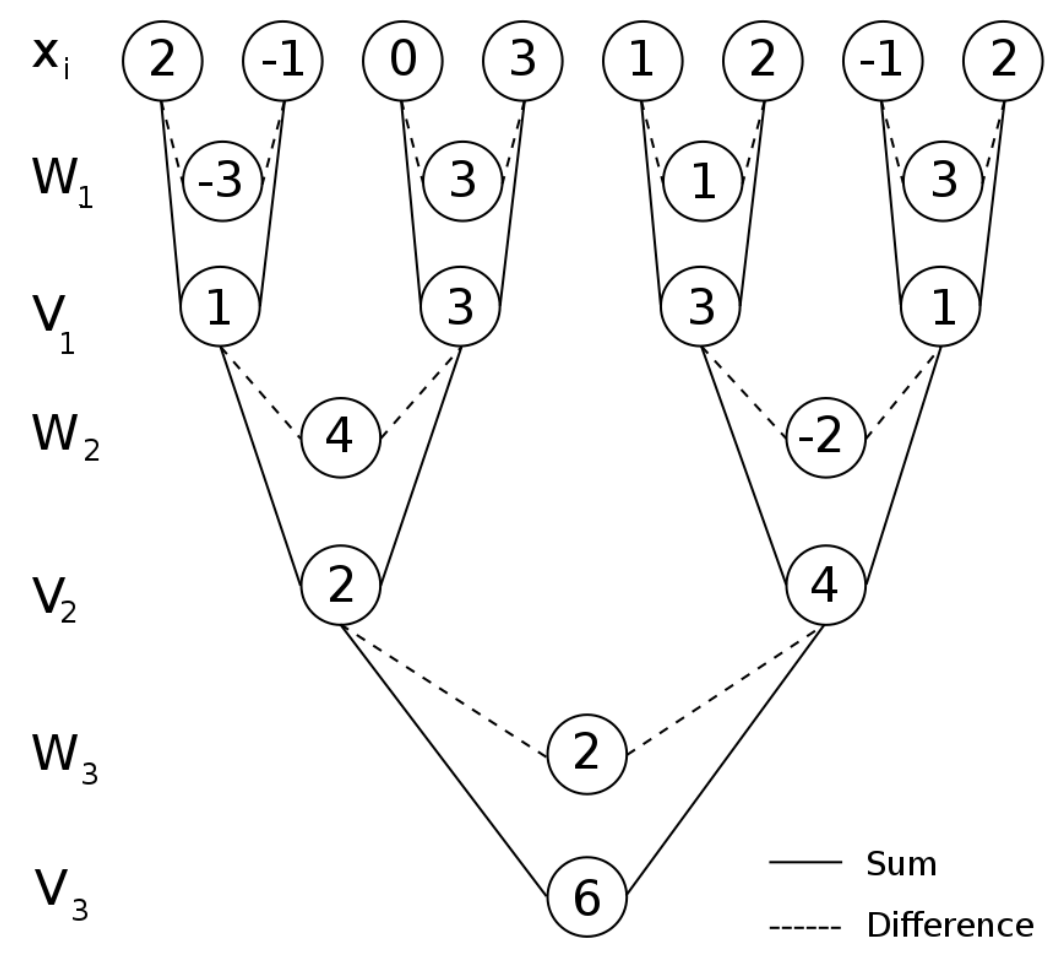

Figure 2.8. Example of DWT Calculation.

Example 2.5 (Heavisine Function, Morettin (2014)). Another example, also from Morettin (2014), is the DWT for the Heavisine function,

$$
f(t)=\sin (2 \pi t)-\frac{1}{2} \cdot \operatorname{sign}(x-0.3)-\frac{1}{2} \cdot \operatorname{sign}(0.72-x), 0 \leq x \leq 1,
$$

illustrated in Figure 2.9. Figure 2.10 presents the DWT of the Heavisine function. We can see that most of the energy of DWT coefficients is concentrated on the smoother scales and the more detailed scales have very small coefficients. 


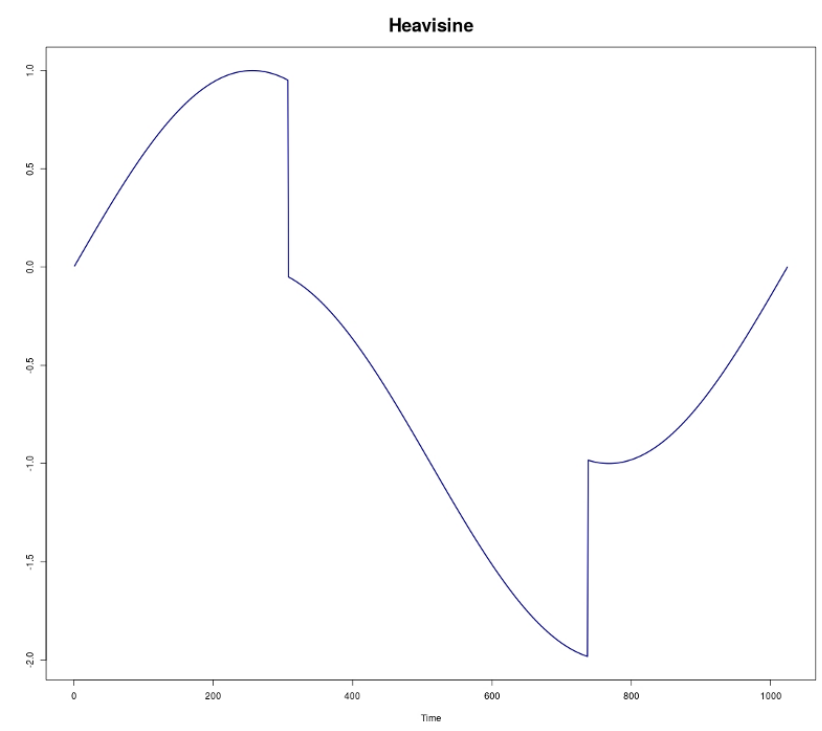

Figure 2.9. Heavisine function.

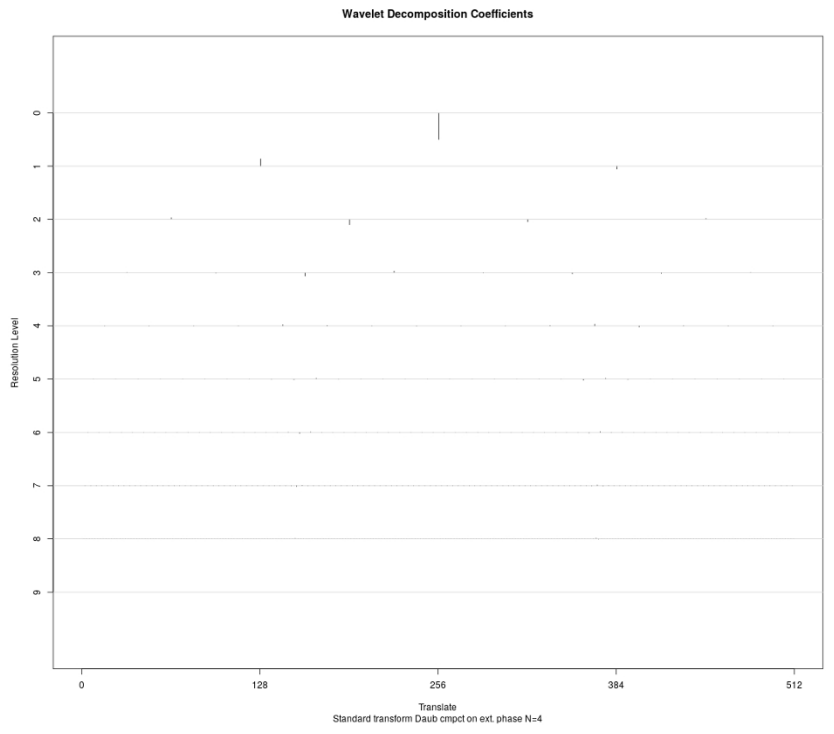

Figure 2.10. Example of DWT Calculation for heavisine function.

\subsubsection{Maximal Overlap Discrete Wavelet Transform}

Maximal Overlap Discrete Wavelet Transform (MODWT) filters are a scaled non-decimated version of the DWT filters. MODWT is also known as non-decimated DWT and unlike DWT, does not need a sample size equal to $2^{J}$. Another important difference is that the number of coefficients in any scale is always T, which makes the MODWT a redundant non-orthonormal transformation. On the other hand, MODWT coefficients are the result of zero-phase filtering, which makes direct the line-up between the coefficients' features from the wavelet domain to the original process in time domain.

Another important difference from DWT to MODWT comes from the redundancy: MODWT mitigates the effects of changing the starting point where the process is sampled. To illustrate this property, we present the following example from Percival and Walden (2000). 
Example 2.6 (Percival and Walden (2000), adapted). Consider a time series $X_{t}$ sampled in $T=128$ and its version shifted by five time units by using operator $\mathcal{T}^{5}$ previously defined. $X_{t}$ consists of a bump' extending over six values surrounded by zeros:

$$
X_{t, 128}=\left\{\begin{array}{cl}
0.5 \cos \left(\frac{3 \pi t}{16}+0.08\right), & t=40, \ldots, 45 \\
0, & t=0, \ldots, 39, \text { and } 46, \ldots, 127 .
\end{array}\right.
$$

Figure 2.11 presents both time series $X_{t}$ and its shifted version $\mathcal{T}^{5} X_{t}$. Figures 2.12 and 2.13 present

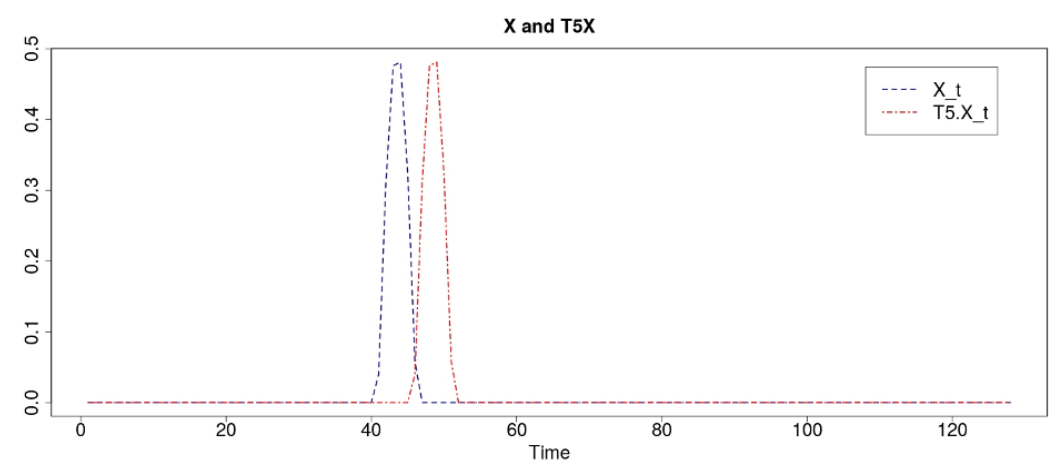

Figure 2.11. Time series for Example 2.6. The two time series are the same, except that $\mathcal{T}^{5} X_{t}$ is a delayed version of $X_{t}$ in five time units.

the DWT and MODWT for both time series using Daubechies wavelet LA(8) and $J_{0}=4$ on the MRA. Notice that the DWT coefficients differs from the original time series $X_{t}$ to the shifted version. On the other hand, the MODWT coefficients are the same, except that these from $\mathcal{T}^{5} X_{t}$ are also shifted. This shift also illustrates the line-up property of the MODWT coefficients with the original time series.

We now formalize the filter theory for the MODWT and present its relation to DWT filters. MODWT filters are a scaled version of DWT. For the $j$-th level, considering DWT filters $\left\{h_{j, l}\right\}$ and $\left\{g_{j, l}\right\}$ defined in (2.40) and (2.41) respectively, the MODWT filters are given by:

$$
\begin{aligned}
\widetilde{h}_{j, l} & \equiv h_{j, l} / 2^{j / 2} \\
\widetilde{g}_{j, l} & \equiv g_{j, l} / 2^{j / 2}
\end{aligned}
$$

From the properties (2.36) and (2.37) we have:

$$
\begin{array}{lll}
\sum_{l=0}^{L_{j}-1} \widetilde{h}_{j, l}=0 ; & \sum_{l=0}^{L_{j}-1} \widetilde{h}_{j, l}^{2}=\frac{1}{2^{j}} ; & \sum_{l=0}^{L_{j}-1} \widetilde{h}_{j, l} \widetilde{h}_{j, l+2^{j} n}=\sum_{l=-\infty}^{\infty} \widetilde{h}_{j, l} \widetilde{h}_{j, l+2^{j} n}=0, \\
\sum_{l=0}^{L_{j}-1} \widetilde{g}_{j, l}=1 ; & \sum_{l=0}^{L_{j}-1} \widetilde{g}_{j, l}^{2}=\frac{1}{2^{j}} ; & \sum_{l=0}^{L_{j}-1} \widetilde{g}_{j, l} \widetilde{g}_{j, l+2^{j} n}=\sum_{l=-\infty}^{\infty} g_{j, l} \widetilde{g}_{j, l+2^{j} n}=0,
\end{array}
$$

for all nonzero integers $n, \widetilde{h}_{j, 0} \neq 0$ and $\widetilde{h}_{j, L_{j}-1} \neq 0$. The MODWT wavelet coefficient $\widetilde{W}_{j, t}$ and 
DWT of X using s8 filters

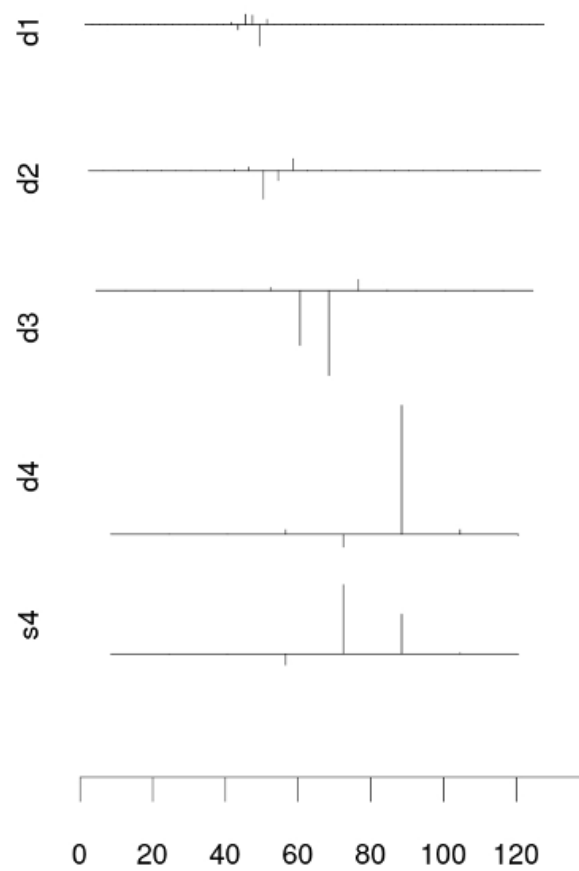

DWT of T5X using s8 filters

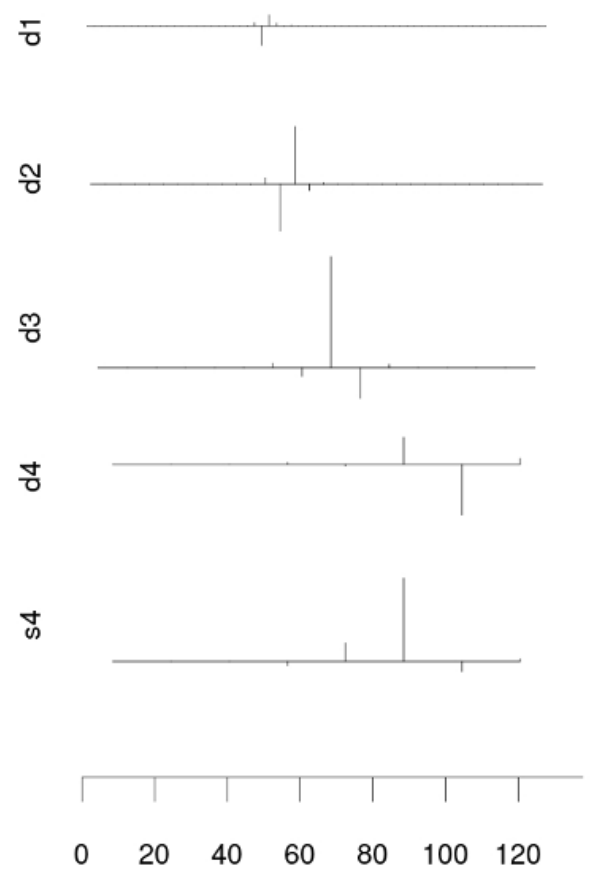

Figure 2.12. Impact of shifting the time series in DWT coefficients: the coefficients differ from the original time series ( $X_{t}$, graphs in the left column) to the shifted time series $\left(\mathcal{T}^{5} X_{t}\right.$, graphs in the right column).

scaling coefficients $\widetilde{V}_{j, t}$ are defined from (2.38) and (2.39) as:

$$
\begin{aligned}
\widetilde{W}_{j, t} & \equiv \sum_{l=0}^{L_{j}-1} \widetilde{h}_{j, l} X_{t-l \operatorname{Mod} T} \\
\widetilde{V}_{j, t} & \equiv \sum_{l=0}^{L_{j}-1} \widetilde{g}_{j, l} X_{t-l \operatorname{Mod} T}
\end{aligned}
$$

with $t=0, \ldots, T-1$ and $L_{j}=\left(2^{j}-1\right)(L-1)+1$. Notice that there is redundancy of information on MODWT due to the non-decimation of the process during the coefficients' calculation. In other words, instead of using only $\left\{X_{\kappa} \mid \kappa=2^{j}(t+1)-1-l \operatorname{Mod} T\right\}$ as in DWT, MODWT uses $\left\{X_{\kappa} \mid \kappa=t-l \operatorname{Mod} T\right\}$. Thus, all scales have $T$ coefficients, and $T$ does not need to be $T=2^{J}, J \in \mathbb{N}^{*}$.

We now recall Example 2.4 to compare the results from DWT with the MODWT. Figure 2.14 illustrates $\widetilde{W}_{j, k}$ and $\widetilde{V}_{0, k}$ calculation. It is clear from this example how circularity assumption affects the coefficients. Comparing with Figure 2.8, is also evident how the non-decimation appears in the calculation of the MODWT.

Figure 2.15 presents the MODWT of the Heavisine function. As we can see, all scales have the same number of coefficients, $T=1024$ in this case. As in DWT, we have the smoother scales concentrating 
MODWT of X using s8 filters
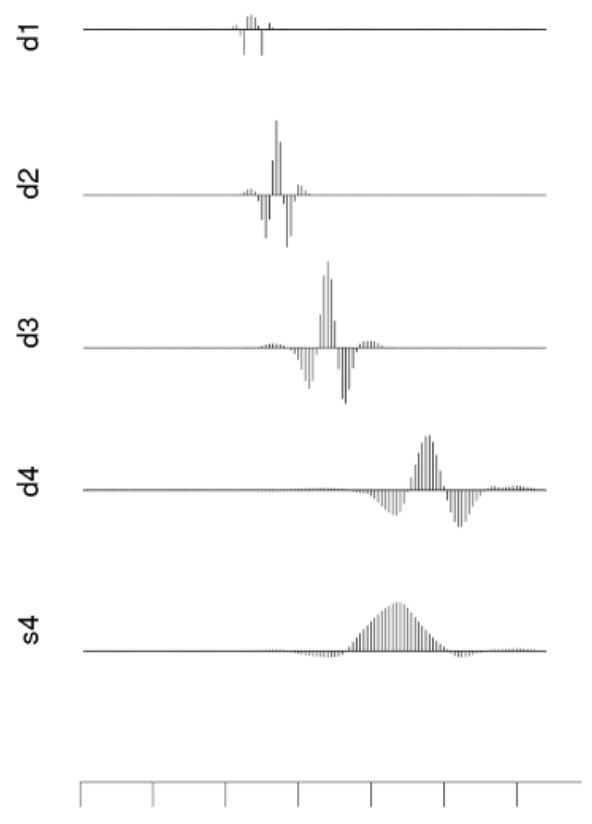

$\begin{array}{lllllll}0 & 20 & 40 & 60 & 80 & 100 & 120\end{array}$
MODWT of T5X using s 8 filters
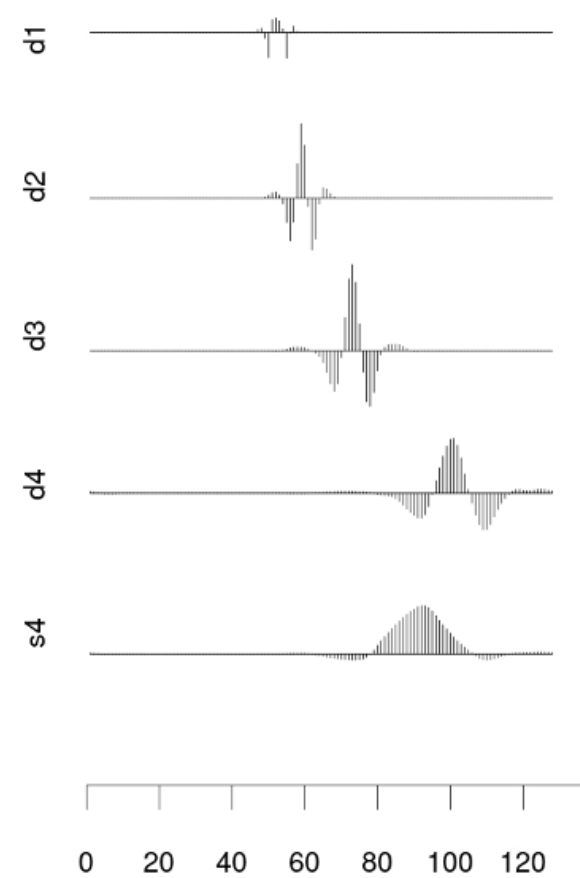

Figure 2.13. Impact of shifting the time series in MODWT coefficients: the coefficients of the $X_{t}$ (left column) and $\mathcal{T}^{5} X_{t}$ (right column) are the same, except for the shift of $\mathcal{T}^{5} X_{t}$ that is reflected on its coefficients.

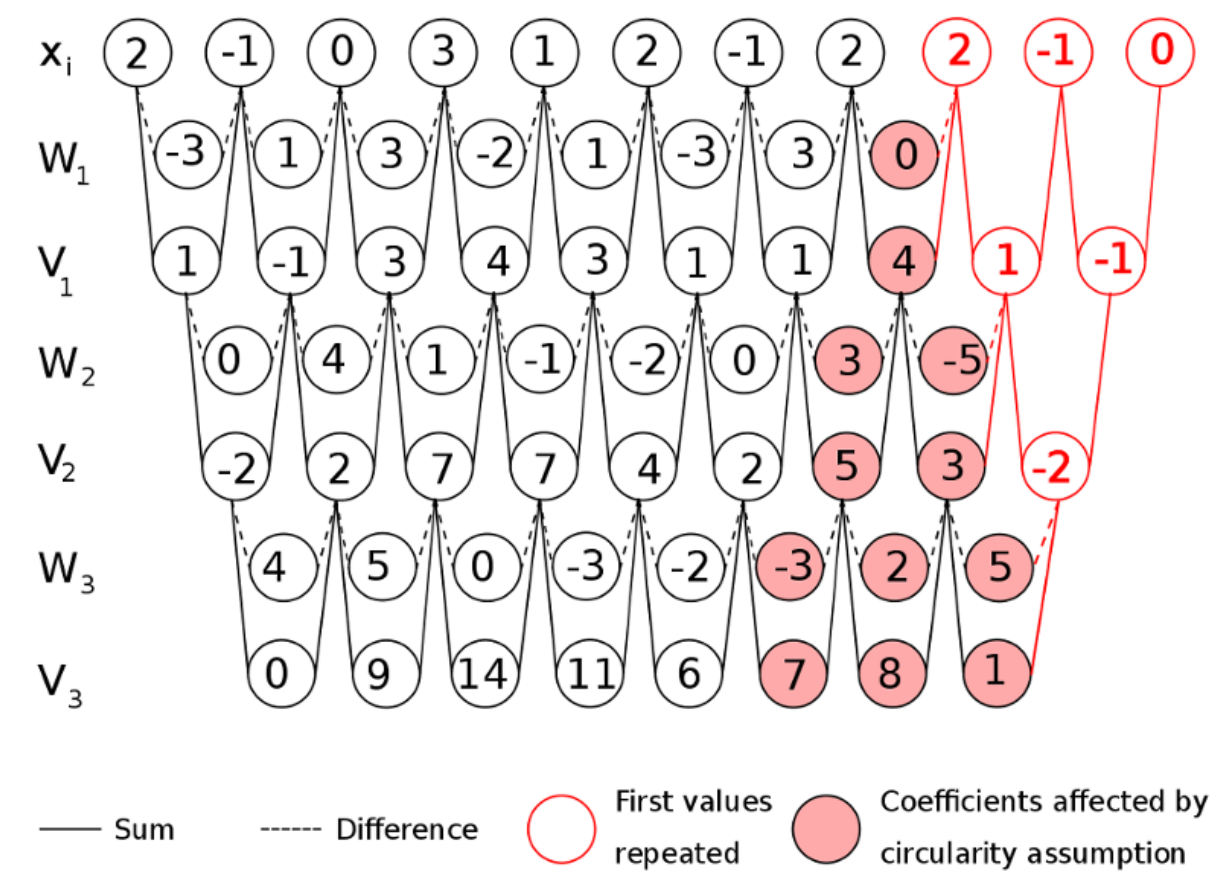

Figure 2.14. Example of MODWT Calculation. 
the major part of the energy of the time series.

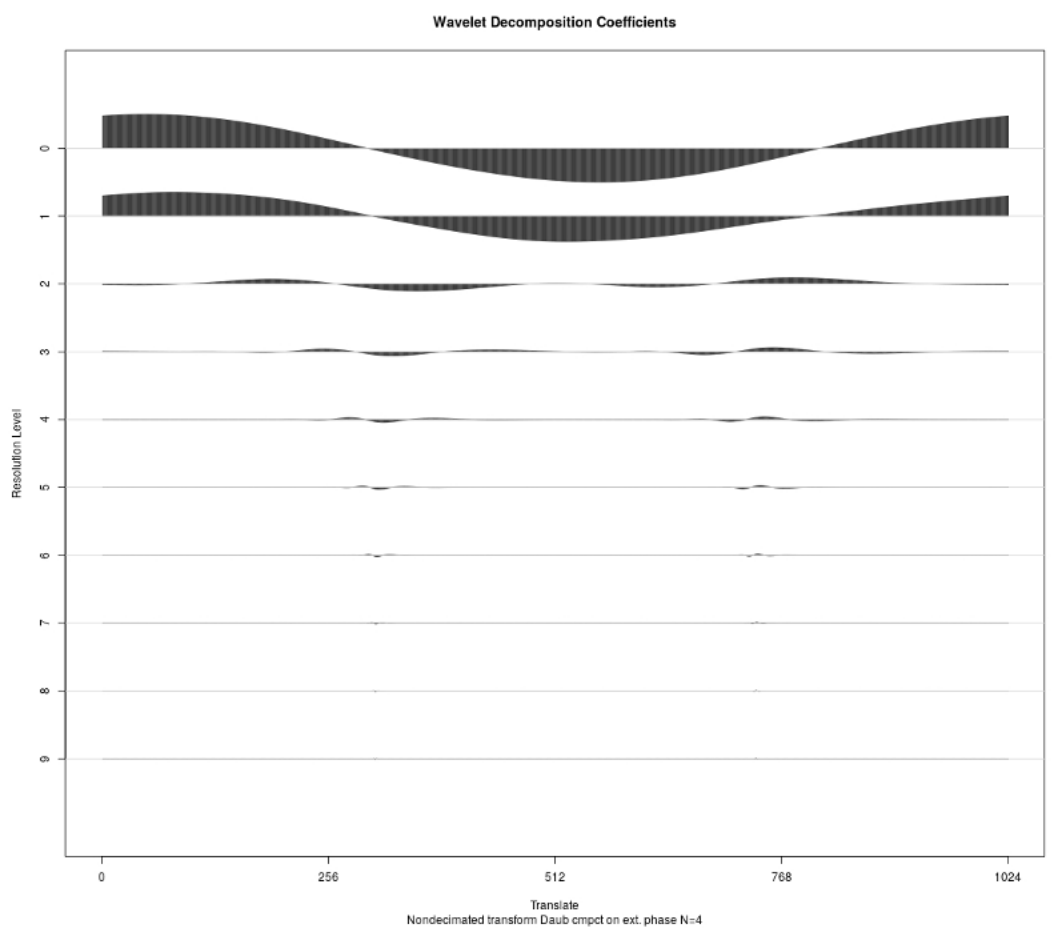

Figure 2.15. Example of MODWT Calculation for heavisine function.

\subsection{Wavelet Covariance}

The key point of this section is to define the Wavelet Covariance - a representation of the covariance between $X_{t, T}$ and $Y_{t, T}$ in the wavelet domain - so we can propose its decomposition in forward and backward signals. Thus, consider $X_{t, T}$ a real-valued locally stationary process. Let $\left\{\widetilde{h}_{j, l}, l=0, \ldots, L_{j}-1\right\}$ be the $j$-th level MODWT wavelet filter associated with scale $\tau_{j} \equiv 2^{j-1}$. Then

$$
\widetilde{W}_{j, t}^{X}=\sum_{l=0}^{L_{j}-1} \widetilde{h}_{j, l} X_{t-l \operatorname{Mod} T}, t=1, \ldots, T
$$

is the stochastic process obtained by filtering $X_{t, T}$ with the wavelet filter $\left\{\widetilde{h}_{j, l}\right\}$. A similar definition holds to $\widetilde{W}_{j, t}^{Y}$.

Definition 2.8. Suppose $X_{t, T}$ is a locally stationary process that satisfies all assumptions in Definition 2.6. If the quantity

$$
\nu_{X, t}^{2}\left(\tau_{j}\right) \equiv \operatorname{var}\left(\widetilde{W}_{j, t}^{X}\right)
$$

exists and is finite, it is called time-dependent wavelet variance.

If we have a stationary process $Z_{t}, t \in \mathbb{Z}$ with Fourier Spectral Density Function $S_{Z}(\omega), \omega \in$ $[-1 / 2,1 / 2]$, then the property

$$
\int_{-1 / 2}^{1 / 2} d S_{Z}(\omega)=\sigma_{Z}^{2}=\operatorname{var}\left(Z_{t}\right)
$$


is valid. For the wavelet variance, the analogous result regarding the variance decomposition is

$$
\sum_{j=1}^{\infty} \nu_{X, t}^{2}\left(\tau_{j}\right)=\sigma_{X}^{2}(t)
$$

i.e., $\nu_{X, t}^{2}\left(\tau_{j}\right)$ decomposes the variance along the scales $\tau_{j}, j \in\{1,2, \ldots\}$.

Definition 2.9. Let $\widetilde{W}_{j, t}^{X}$ and $\widetilde{W}_{j, t}^{Y}$ defined in (2.44). Then, the quantity

$$
\nu_{X Y, t}\left(\tau_{j}\right)=\operatorname{Cov}\left(\widetilde{W}_{j, t}^{X}, \widetilde{W}_{j, t}^{Y}\right),
$$

for $t=1, \ldots, T, j=1, \ldots, J$, is called the Wavelet Covariance $(W C)$ of $\widetilde{W}_{j, t}^{X}$ and $\widetilde{W}_{j, t}^{Y}$.

The WC $\nu_{X Y, t}\left(\tau_{j}\right)$ yields a scale-based representation of the covariance between $X_{t, T}$ and $Y_{t, T}$ (Percival and Walden (2000)):

$$
\sum_{j=1}^{\infty} \nu_{X Y, t}\left(\tau_{j}\right)=\operatorname{cov}\left(X_{t, T}, Y_{t, T}\right) .
$$

\subsection{Wavelet Coherence}

Cross Wavelet Transform (XWT) and Wavelet Coherence (WTC) (Torrence and Compo (1998) and Grinsted et al. (2004)) offer a different approach to deal with causal relations between nonstationary processes. They are representations of the $X_{t}$ and $Y_{t}$ relation in the wavelet domain using a complex wavelet. The main idea is considering the phase between power signals in the time-scale domain to identify a possible causality relation between two time series.

Let $W^{X}$ be the Continuous Wavelet Transform of $X_{t}$ using a complex wavelet, e.g. Morlet wavelet, and its wavelet power $\left|W^{X}\right|^{2}$ and analogous quantities defined for $Y_{t}$. Then the Cross Wavelet Transform is defined as

$$
W^{X Y}=W^{X} W^{Y \star}
$$

where $\star$ denotes complex conjugation. We can interpret the complex part of $W^{X Y}$ as local phases between $X_{t}$ and $Y_{t}$. For significant regions with high cross wavelet power $\left|W^{X Y}\right|$, the local phase estimates the relationship between $X_{t}$ and $Y_{t}: X \rightarrow Y$ when we have positive phases or $X \leftarrow Y$ if the phases are negative.

Grinsted et al. (2004) define Wavelet Coherence (WTC) as

$$
R_{j}^{2}(t)=\frac{\left|S\left(t^{-1} W_{j}^{X Y}(t)\right)\right|^{2}}{S\left(t^{-1}\left|W_{j}^{X}(t)\right|^{2}\right) \cdot S\left(t^{-1}\left|W_{j}^{Y}(t)\right|^{2}\right)}, t \in \mathbb{Z},
$$

and $S($.$) is a smoothing operator along both time and scale spaces: S(W)=S_{\text {scale }}\left(S_{\text {time }}\left(W_{j}(t)\right)\right)$. We can interpret this measure as a correlation in the time-scale space and its significance can be estimated by Monte Carlo Methods (Grinsted et al. (2004)).

For instance, Figure 2.16 from Example 2.3 presents the WTC for the three relations $X_{1} \leftrightarrow X_{2}$, 
$X_{1} \leftrightarrow X_{3}$ and $X_{2} \leftrightarrow X_{3}$. Notice the red regions with the arrows. They represent the time-scales where the WTC is different from the WTC of a stationary VAR(1), using Monte Carlo simulation. The top of each graph represents the more detailed scales while at the bottom we have the smoothed scales. Finally, the arrows represent the phases of the WTC, i.e., $\arg \left(R_{j}(t)\right)$.
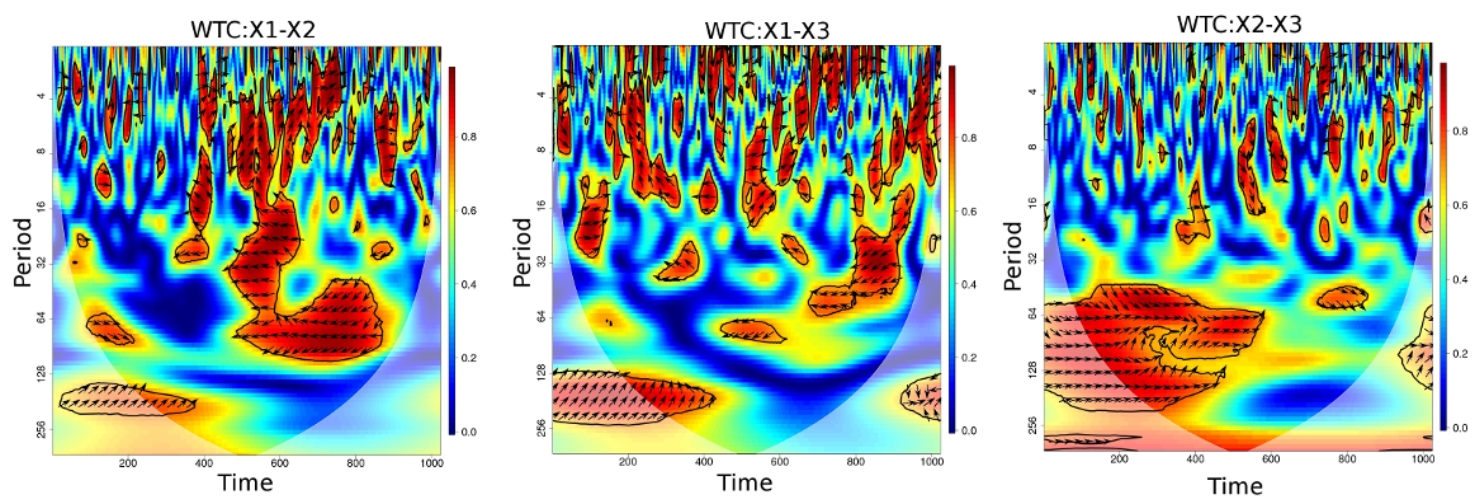

Figure 2.16. Wavelet Coherence for Example 2.3. The red regions represent the time-scale combination in which estimated WTC is different with 5\% of significance from a stationary VAR(1) WTC using Monte Carlo simulation. The arrows inside the regions are the phases between $W_{j}^{X}(t)$ and $W_{j}^{Y}(t)$. If the arrow points to $-\pi / 2 \mathrm{rad}$ then we have a very strong relation $X \rightarrow Y$ and $Y \rightarrow X$ if it points to $\pi / 2 \mathrm{rad}$.

In the next chapter, considering locally stationary processes as presented in Definition 2.6, we propose a decomposition of the Wavelet Covariance (2.45) in forward and backward signals: the Directed Wavelet Covariance. Like Parseval's relation (2.2) for Discrete Fourier Transform, the relation (2.46) preserves the process' energy for wavelet transforms. Moreover, unlike Fourier transforms, wavelet transforms have a time-scale multilevel (2.34) index, which gives us multiple scale resolution to evaluate whether the relations between the time series show short and/or long-term variations. We also present properties and examples of the proposed decomposition factors. Comparisons with Partial Directed Coherence (2.19) and Wavelet Coherence (2.47) will be presented later in Chapter 5. 


\section{Chapter 3}

\section{Directed Wavelet Covariance}

In this chapter we present the first contribution of the study. The previous chapter presented common techniques used to analyze data with directed measures. We have shown that Fourier analysis may not be the best option to non-stationary processes and have introduced wavelet analysis and wavelet covariance, that will be the basis for this chapter and Chapter 4.

We start this chapter stating the wavelet covariance decomposition into forward and backward signals. We discuss these quantities and present some examples. Next, we propose estimators for these two quantities and calculate their expectation and an upper limit for their variances.

In Chapter 5 we will show some simulations with this decomposition and compare the results with known quantities as PDC and WTC from the Chapter 2. One example using real data will be presented in Chapter 6. We will analyze electroencephalogram (EEG) data to evaluate three channels relations during imaginary movements experiment.

Proposition 3.1. Consider $X_{t, T}$ and $Y_{t, T}$ two real-valued locally stationary stochastic processes that admit the time-varying $M A(\infty)$ representation (2.26) with $\mathbb{E}\left(X_{t, T}\right)=\mu_{X}(u), \mathbb{E}\left(Y_{t, T}\right)=$ $\mu_{Y}(u), t=1, \ldots, T, u=t / T$. Let $\left\{\widetilde{h}_{j, l}\right\}$ be the $j$-th level of MODWT filter associated with scale $\tau_{j}$ and $\nu_{X Y, t}\left(\tau_{j}\right)$ the wavelet covariance defined in (2.45). If $L_{j}=\left(2^{j}-1\right)\left(L_{1}-1\right)+1$ is the width of the filter ( $L_{1}$ is the width of the unit scale filter $\left.h_{l}=h_{1, l}\right), T \geq L_{j}$ and $\gamma_{X Y}\left(t_{1}, t_{2}\right)=\operatorname{Cov}\left(X_{t_{1}}, Y_{t_{2}}\right)$, then the wavelet covariance $\nu_{X Y, t}\left(\tau_{j}\right)$ can be written as:

$$
\nu_{X Y, t}\left(\tau_{j}\right)=F_{X Y, t}\left(\tau_{j}\right)+D_{X Y, t}\left(\tau_{j}\right)+B_{X Y, t}\left(\tau_{j}\right)+\widetilde{W}_{j, t}\left(\mu_{X}(u)\right) \widetilde{W}_{j, t}\left(\mu_{Y}(u)\right),
$$

with

$$
\begin{aligned}
F_{X Y, t}\left(\tau_{j}\right) & =\sum_{l=1}^{L_{j}-1} \sum_{l^{\prime}=0}^{l-1} \widetilde{h}_{j, l}\left(\widetilde{h}_{j, l^{\prime}}\right)^{H} \gamma_{X Y}\left(t-l, t-l^{\prime}\right), \\
D_{X Y, t}\left(\tau_{j}\right) & =\sum_{l=0}^{L_{j}-1} \widetilde{h}_{j, l}\left(\widetilde{h}_{j, l}\right)^{H} \gamma_{X Y}(t-l, t-l), \\
B_{X Y, t}\left(\tau_{j}\right) & =\sum_{l=0}^{L_{j}-2} \sum_{l^{\prime}=l+1}^{L_{j}-1} \widetilde{h}_{j, l}\left(\widetilde{h}_{j, l^{\prime}}\right)^{H} \gamma_{X Y}\left(t-l, t-l^{\prime}\right) .
\end{aligned}
$$

Proof. See Appendix B. 
Therefore, the Wavelet Covariance (2.45) can be decomposed into 4 factors: $F_{X Y, t}\left(\tau_{j}\right), D_{X Y, t}\left(\tau_{j}\right)$ and $B_{X Y, t}\left(\tau_{j}\right)$ representing the causal relations between $X_{t, T}$ and $Y_{t, T}$, plus the term related with the average functions of both signals: $\widetilde{W}_{j, t}\left(\mu_{X}(u)\right) \widetilde{W}_{j, t}\left(\mu_{Y}(u)\right)$. While $F_{X Y, t}\left(\tau_{j}\right)$ and $B_{X Y, t}\left(\tau_{j}\right)$ measure the impact of the past $X_{t, T}$ in the future $Y_{t, T}$, and the past $Y_{t, T}$ in the future $X_{t, T}$ respectively , $D_{X Y, t}\left(\tau_{j}\right)$ represent the relation between these two processes when they are at the same time $t$ (instantaneous causality). $\widetilde{W}_{j, t}\left(\mu_{X}(u)\right) \widetilde{W}_{j, t}\left(\mu_{Y}(u)\right)$ is the product of the DWTs of $X_{t, T}$ and $Y_{t, T}$ average functions and will be zero if $\mu_{X}(u) \equiv 0$ or $\mu_{Y}(u) \equiv 0$.

\subsection{Estimation}

The covariance function $\gamma_{X Y}(t, l)$ for non-stationary processes does not depend only of $\mid t-$ $l \mid$. Therefore the stationary properties and estimators of $\gamma_{X Y}$ are not valid. Priestley (1965) and Dahlhaus (1996) discussed the estimation of this function and by considering the proposed estimator in Dahlhaus (1996) we estimate the covariance function, $c_{T}(u, k)=\int_{-\pi}^{\pi} f(u, k) \exp (i \omega k) d \omega$, by:

$$
\hat{c_{T}}(u, k)=\frac{1}{b_{T} T} \sum_{s} K\left(\frac{u T-(s+k / 2)}{b_{T} T}\right) X_{s, T} Y_{s+k, T}
$$

where $K($.$) is a zero-centered, symmetric Kernel K: \mathbb{R} \rightarrow[0, \infty), \int K(x) d x=1$ and $K(x)=0$ if $x \notin[-1 / 2,1 / 2], b_{T}$ is the bandwidth and $u=t / T, t=1, \ldots, T$.

Hence we propose the estimators for the quantities (3.1) and (3.2) as follows:

$$
\begin{gathered}
\widehat{F}_{X Y, t}\left(\tau_{j}\right)=\sum_{l=1}^{L_{j}-1} \sum_{l^{\prime}=0}^{l-1} \widetilde{h}_{j, l}\left(\tilde{h}_{j, l^{\prime}}\right)^{H}\left[\hat{c}_{T}\left(\frac{(t-l)}{T},\left(l^{\prime}-l\right)\right)\right], \\
\widehat{B}_{X Y, t}\left(\tau_{j}\right)=\sum_{l=0}^{L_{j}-2} \sum_{l^{\prime}=l+1}^{L_{j}-1} \widetilde{h}_{j, l}\left(\tilde{h}_{j, l^{\prime}}\right)^{H}\left[\hat{c}_{T}\left(\frac{(t-l)}{T},\left(l^{\prime}-l\right)\right)\right] .
\end{gathered}
$$

We want to estimate the causal relation between two processes $X_{t, T}$ and $Y_{t, T}$. Given (3.3) and (3.4), at some point during estimation of $F_{X Y, t}\left(\tau_{j}\right)$ a proxy to the directed covariance between past $X_{t}$ 's and future of $Y_{t}$ 's will be

$$
\sum_{l=1}^{L_{j}-1} \sum_{l^{\prime}=0}^{l-1} \widetilde{h}_{j, l}\left(\widetilde{h}_{j, l}\right)^{H} X_{t-l M o d T} Y_{t-l^{\prime} M o d T}, t=1, \ldots, T .
$$

But since $L_{j}=\left(2^{j}-1\right)\left(L_{1}-1\right)+1,(t-l M o d T)$ can be bigger than $T$ for some $j$ and $t$. Furthermore in situations with $t<L_{j}$ or $t>T-L_{j}$, we can find a set of pairs $\left\{\left(l, l^{\prime}\right)\right\}$ such that:

$$
X_{t-l \operatorname{Mod} T} Y_{t-l^{\prime} \operatorname{Mod} T}=X_{l-t} Y_{t-l^{\prime}}=X_{a} Y_{t-l^{\prime}}
$$

with $a>t-l^{\prime}$.

This directly relates past $Y_{t}$ 's with future $X_{t}$ 's and should not contribute to the estimation of the relation $X \rightarrow Y$. Therefore we discard coefficients involving circular convolution since they may lead 
us to misinterpreter the causality structure. Notice that for $L_{1}=2$ we have $L_{J}=T$ and the affected coefficients occur only on the boards - the first and the last $L_{j}$ coefficients - of scale $j<J$ and all coefficients of scale $\tau_{J}$.

\subsubsection{Properties of the Estimators: Expectation and Variance}

From Dahlhaus (1996) we have:

$$
\mathbb{E}\left[\hat{c}_{T}(u, k)\right]=c(u, k)+(1 / 2) b_{T}^{2} \int x^{2} K(x) d x\left[\frac{\partial^{2}}{\partial u^{2}} c(u, k)\right]+o\left(b_{T}^{2}\right)+O\left(\frac{1}{b_{T} T}\right)
$$

which is biased due to the non-stationary characteristic of the process, measured by $\frac{\partial^{2}}{\partial u^{2}} c(u, k)$. This bias is zero if the process is stationary. We present now two propositions regarding expectation and variance of $\widehat{F}_{X Y, t}\left(\tau_{j}\right)$ and $\widehat{B}_{X Y, t}\left(\tau_{j}\right)$.

Proposition 3.2. Consider the estimators $\widehat{F}_{X Y, t}\left(\tau_{j}\right)$ and $\widehat{B}_{X Y, t}\left(\tau_{j}\right)$ defined in (3.4) and (3.5) respectively. If the covariance estimator (3.3) has expected value given by (3.6) then:

$$
\begin{aligned}
\mathbb{E}\left[\widehat{F}_{X Y, t}\left(\tau_{j}\right)\right]= & F_{X Y, t}\left(\tau_{j}\right)+\frac{1}{2} b_{T}^{2} \int x^{2} K(x) d x \sum_{l=1}^{L_{j}-1} \sum_{l^{\prime}=0}^{l-1} \widetilde{h}_{j, l}\left(\tilde{h}_{j, l^{\prime}}\right)^{H}\left(\frac{\partial^{2}}{\partial t^{2}} c\left(t+l, l-l^{\prime}\right)\right) \\
& +o\left(b_{T}^{2}\right)+O\left(\frac{1}{b_{T} T}\right), \\
\mathbb{E}\left[\widehat{B}_{X Y, t}\left(\tau_{j}\right)\right]= & B_{X Y, t}\left(\tau_{j}\right)+(1 / 2) b_{T}^{2} \int x^{2} K(x) d x \sum_{l=1}^{L_{j}-1} \sum_{l^{\prime}=0}^{l-1} \widetilde{h}_{j, l}\left(\tilde{h}_{j, l^{\prime}}\right)^{H}\left(\frac{\partial^{2}}{\partial t^{2}} c\left(t+l, l-l^{\prime}\right)\right) \\
& +o\left(b_{T}^{2}\right)+O\left(\frac{1}{b_{T} T}\right) .
\end{aligned}
$$

Proof. See Appendix B.

As we can see, the bias is transferred to the wavelet domain, and affects the estimation of $F($. and $B($.$) as well.$

Proposition 3.3. Consider $X_{t, T}$ and $Y_{t, T}$ locally stationary processes with $T<\infty$, such that $Z_{k}=$ $\sum_{s} X_{s} Y_{s-k}, k<\infty$ is a process with finite variance. If $K($.$) used in the covariance estimator$ (3.3) is the Epanechnikov kernel, then the estimators $\widehat{F}_{X Y, t}\left(\tau_{j}\right)(3.4)$ and $\widehat{B}_{X Y, t}\left(\tau_{j}\right)$ (3.5) have finite variances with upper limits given by

$$
\begin{aligned}
& \operatorname{Var}\left[\widehat{F}_{X Y, t}\left(\tau_{j}\right)\right] \leq \frac{9 \max _{l}\left\{\left|\widetilde{h}_{j, l}\right|\right\}^{4}}{4\left(b_{T} T\right)^{2}} \operatorname{Var}\left\{\sum_{l^{*}=1}^{L_{j}-1}\left(L_{j}-l^{*}\right) \sum_{s} X_{s} Y_{s-l^{*}}\right\}, \\
& \operatorname{Var}\left[\widehat{B}_{X Y, t}\left(\tau_{j}\right)\right] \leq \frac{9 \max _{l}\left\{\left|\widetilde{h}_{j, l}\right|\right\}^{4}}{4\left(b_{T} T\right)^{2}} \operatorname{Var}\left\{\sum_{l^{*}=1}^{L_{j}-1}\left(L_{j}-l^{*}\right) \sum_{s} X_{s} Y_{s-l^{*}}\right\}
\end{aligned}
$$

Proof. See Appendix B. 
We will use these estimators in Chapters 5 and 6 . They will help us to evaluate the simulated processes behavior and to study the EEG collected data.

To better understand how DWC behaves and how close their estimators are from their real values, we present four examples of stationary situations in which we can calculate the theoretical covariance function. These examples also will help us to propose statistical tests to evaluate the significance of EEG signals in Chapter 6 . In Chapter 5 we present examples of locally stationary processes and compare the DWC results with other studied quantities.

Example 3.1 (VAR(1) with independent components). We first consider the simplest example of a stationary bivariate Vector Autoregressive model of order 1, VAR(1), with uncorrelated components, i.e. we consider the model with the form:

$$
\begin{gathered}
\mathbf{X}_{t}=\mathbf{A X}_{t-1}+\boldsymbol{\epsilon}(t) \\
\left(\begin{array}{l}
X_{1, t} \\
X_{2, t}
\end{array}\right)=\mathbf{A}\left(\begin{array}{l}
X_{1, t-1} \\
X_{2, t-1}
\end{array}\right)+\left(\begin{array}{l}
\epsilon_{1, t} \\
\epsilon_{2, t}
\end{array}\right), \\
\mathbf{A}=\left(\begin{array}{ll}
a_{11} & a_{12} \\
a_{21} & a_{22}
\end{array}\right)=\left(\begin{array}{ll}
a & 0 \\
0 & b
\end{array}\right),
\end{gathered}
$$

$\boldsymbol{\epsilon}(t)=\left(\begin{array}{c}\epsilon_{1, t} \\ \epsilon_{2, t}\end{array}\right) \sim W N \underset{\sim}{(0, \boldsymbol{\Sigma})}$ is a bivariate white noise with independent components and covariance matrix $\boldsymbol{\Sigma}=\left(\begin{array}{cc}\operatorname{Var}\left(\epsilon_{1, t}\right) & 0 \\ 0 & \operatorname{Var}\left(\epsilon_{2, t}\right)\end{array}\right)=I_{2}$, and the stationarity conditions hold to $a$ and $b$ i.e. $|a|<1,|b|<1$.

We know that (Lütkepohl (2007) and Morettin (2014)) the covariance matrix $\Gamma(k)$ for this process can be calculated as follows:

$$
\begin{array}{ll}
\Gamma(k) & =\operatorname{Cov}\left(\mathbf{X}_{1, t}, \mathbf{X}_{2, t-k}\right)=\phi_{1} \Gamma(k-1), \\
\operatorname{vec}(\Gamma(0)) & =\left(I_{k}^{2}-\mathbf{A} \otimes \mathbf{A}\right)^{-1} \operatorname{vec}(\boldsymbol{\Sigma}), \\
\Gamma(k)=\Gamma(-k)^{\top} . &
\end{array}
$$

Therefore in this case we have:

$$
\begin{aligned}
& \operatorname{vec}(\Gamma(0))=\left(I_{k}^{2}-\mathbf{A} \otimes \mathbf{A}\right)^{-1} \operatorname{vec}(\boldsymbol{\Sigma})=\left(\begin{array}{cccc}
\frac{1}{1-a^{2}} & 0 & 0 & 0 \\
0 & \frac{1}{1-a^{2}} & 0 & 0 \\
0 & 0 & \frac{1}{1-a^{2}} & 0 \\
0 & 0 & 0 & \frac{1}{1-b^{2}}
\end{array}\right)\left(\begin{array}{llll}
1 & 0 & 0 & 1
\end{array}\right)= \\
& =\left(\gamma_{11}(0), 0, \quad 0, \gamma_{22}(0)\right) \text {. }
\end{aligned}
$$


Thus,

$$
\begin{aligned}
\Gamma(0) & =\left(\begin{array}{cc}
\gamma_{11}(0) & 0 \\
0 & \gamma_{22}(0)
\end{array}\right) \\
\Gamma(1) & =A \cdot \Gamma(0)=\left(\begin{array}{ll}
a & 0 \\
0 & b
\end{array}\right) \cdot\left(\begin{array}{cc}
\gamma_{11}(0) & 0 \\
0 & \gamma_{22}(0)
\end{array}\right)=\left(\begin{array}{cc}
a \gamma_{11}(0) & 0 \\
0 & b \gamma_{22}(0)
\end{array}\right) \\
\Gamma(2) & =A . \Gamma(1)=\left(\begin{array}{ll}
a & 0 \\
0 & b
\end{array}\right) \cdot\left(\begin{array}{cc}
\gamma_{11}(1) & 0 \\
0 & \gamma_{22}(1)
\end{array}\right)=\left(\begin{array}{cc}
a^{2} \gamma_{11}(0) & 0 \\
0 & b^{2} \gamma_{22}(0)
\end{array}\right) \\
\vdots & \\
\Gamma(k) & =A \cdot \Gamma(k-1)=\left(\begin{array}{ll}
a & 0 \\
0 & b
\end{array}\right) \cdot\left(\begin{array}{cc}
\gamma_{11}(1) & 0 \\
0 & \gamma_{22}(1)
\end{array}\right)=\left(\begin{array}{cc}
a^{k} \gamma_{11}(0) & 0 \\
0 & b^{k} \gamma_{22}(0)
\end{array}\right), \forall k \in \mathbb{Z} .
\end{aligned}
$$

Now since $\forall k \in \mathbb{Z}, \gamma_{12}(k)=\gamma_{21}(k)=0$ then $F_{12, t}\left(\tau_{j}\right)=B_{12, t}\left(\tau_{j}\right)=0, \forall j \in\{1, \ldots, J\}$.

Example 3.1 sets an important result: $F_{12, t}\left(\tau_{j}\right)=B_{12, t}\left(\tau_{j}\right)=0$ in a stationary bivariate $\operatorname{VAR}(1)$ with independent components. This result will be important in Chapter 6, where we will define a criteria to measure the significance in EEG signals.

Example 3.2 (VAR(1) with one direction dependency). In this second example of bivariate VAR(1) we have the following process:

$$
\begin{gathered}
\mathbf{X}_{t}=\mathbf{A X}_{t-1}+\boldsymbol{\epsilon}(t) \\
\left(\begin{array}{l}
X_{1, t} \\
X_{2, t}
\end{array}\right)=\mathbf{A}\left(\begin{array}{l}
X_{1, t-1} \\
X_{2, t-1}
\end{array}\right)+\left(\begin{array}{l}
\epsilon_{1, t} \\
\epsilon_{2, t}
\end{array}\right), \\
\mathbf{A}=\left(\begin{array}{ll}
a_{11} & a_{12} \\
a_{21} & a_{22}
\end{array}\right)=\left(\begin{array}{ll}
0 & a \\
0 & b
\end{array}\right),
\end{gathered}
$$

$\boldsymbol{\epsilon}(t), a$ and $b$ as in Example 3.1.

In this example we have the past of $X_{2, t}$ affecting present observations of $X_{1, t}$ but present of $X_{2, t}$ does not depend on $X_{1, t}$ time series. From 3.8 we can calculate $\Gamma(k)$ as follows:

$$
\begin{aligned}
\operatorname{vec}(\Gamma(0)) & =\left(I_{k}^{2}-\mathbf{A} \otimes \mathbf{A}\right)^{-1} \operatorname{vec}(\Sigma)=\left(\begin{array}{cccc}
1 & 0 & 0 & \frac{a}{1-b^{2}} \\
0 & 1 & 0 & \frac{b}{1-b^{2}} \\
0 & 0 & 1 & \frac{b}{1-b^{2}} \\
0 & 0 & 0 & \frac{1}{1-b^{2}}
\end{array}\right)\left(\begin{array}{llll}
1 & 0 & 0 & 1
\end{array}\right)= \\
& =\left(1+\frac{a^{2}}{1-b^{2}}, \quad \frac{a b}{1-b^{2}}, \quad \frac{a b}{1-b^{2}}, \frac{1}{1-b^{2}}\right) .
\end{aligned}
$$

Thus

$\Gamma(0)=\left(\begin{array}{cc}1+\frac{a^{2}}{1-b^{2}} & \frac{a b}{1-b^{2}} \\ \frac{a b}{1-b^{2}} & \frac{1}{1-b^{2}}\end{array}\right)$ 


$$
\begin{aligned}
& \Gamma(1)=A \cdot \Gamma(0)=\left(\begin{array}{ll}
0 & a \\
0 & b
\end{array}\right) \cdot\left(\begin{array}{ll}
\gamma_{11}(0) & \gamma_{12}(0) \\
\gamma_{21}(0) & \gamma_{22}(0)
\end{array}\right)=\left(\begin{array}{ll}
a \gamma_{21}(0) & a \gamma_{22}(0) \\
b \gamma_{21}(0) & b \gamma_{22}(0)
\end{array}\right) \\
& \Gamma(2)=A \cdot \Gamma(1)=\left(\begin{array}{ll}
0 & a \\
0 & b
\end{array}\right) \cdot\left(\begin{array}{ll}
\gamma_{11}(1) & \gamma_{12}(1) \\
\gamma_{21}(1) & \gamma_{22}(1)
\end{array}\right)=\left(\begin{array}{ll}
a b \gamma_{21}(0) & a b \gamma_{22}(0) \\
b^{2} \gamma_{21}(0) & b^{2} \gamma_{22}(0)
\end{array}\right) \\
& \Gamma(k)=A \cdot \Gamma(k-1)=\left(\begin{array}{cc}
0 & a \\
0 & b
\end{array}\right) \cdot\left(\begin{array}{cc}
\gamma_{11}(k-1) & \gamma_{12}(k-1) \\
\gamma_{21}(k-1) & \gamma_{22}(k-1)
\end{array}\right)=\left(\begin{array}{cc}
a b^{k-1} \gamma_{21}(0) & a b^{k-1} \gamma_{22}(0) \\
b^{k} \gamma_{21}(0) & b^{k} \gamma_{22}(0)
\end{array}\right) .
\end{aligned}
$$

Using the property $\Gamma(k)=\Gamma(-k)^{\top}$ then $\gamma_{12}(k)=\gamma_{21}(-k)$. So, the cross-covariance function $\gamma_{12}$ is:

$$
\begin{aligned}
\gamma_{12}(k) & =a b^{k-1} \gamma_{22}(0)=a b^{k-1} \frac{1}{1-b^{2}}, \quad \text { if } k>0, \\
& \text { or } \\
\gamma_{12}(k) & =\gamma_{21}(-k)=b^{-k} \frac{a b}{1-b^{2}}=\frac{a b^{1-k}}{1-b^{2}}, \quad \text { if } k<0 .
\end{aligned}
$$

Now, since the process is stationary, $\gamma_{12}\left(t_{1}, t_{2}\right)=\mathbb{E}\left(X_{1, t_{1}} X_{2, t_{2}}\right)=\mathbb{E}\left(X_{1, t} X_{2, t-k}\right)=\gamma_{12}(k)$, then $k=t_{1}-t_{2}$ and $F_{X Y, t}\left(\tau_{j}\right)$ and $B_{X Y, t}\left(\tau_{j}\right)$ are given as follows:

$$
\begin{aligned}
F_{12, t}\left(\tau_{j}\right)= & \sum_{l=1}^{L_{j}-1} \sum_{l^{\prime}=0}^{l-1} \widetilde{h}_{j, l} \widetilde{h}_{j, l^{\prime}} \gamma_{12}\left(t-l, t-l^{\prime}\right)=\sum_{l=1}^{L_{j}-1} \sum_{l^{\prime}=0}^{l-1} \widetilde{h}_{j, l} \widetilde{h}_{j, l^{\prime}} \mathbb{E}\left(X_{1, t-l} X_{2, t-l^{\prime}}\right)= \\
= & \sum_{l=1}^{L_{j}-1} \sum_{l^{\prime}=0}^{l-1} \widetilde{h}_{j, l} \widetilde{h}_{j, l^{\prime}} \gamma_{12}\left(t-l-\left(t-l^{\prime}\right)\right)=\sum_{l=1}^{L_{j}-1} \sum_{l^{\prime}=0}^{l-1} \widetilde{h}_{j, l} \widetilde{h}_{j, l^{\prime}} \gamma_{12}(\underbrace{l^{\prime}-l}_{=k<0})= \\
= & \sum_{l=1}^{L_{j}-1} \sum_{l^{\prime}=0}^{l-1} \widetilde{h}_{j, l} \widetilde{h}_{j, l^{\prime}} \frac{a b^{1-\left(l^{\prime}-l\right)}}{1-b^{2}}=\frac{a}{1-b^{2}} \sum_{l=1}^{L_{j}-1} \sum_{l^{\prime}=0}^{l-1} \widetilde{h}_{j, l} \widetilde{h}_{j, l^{\prime}} b^{\left(l-l^{\prime}+1\right)} . \\
B_{12, t}\left(\tau_{j}\right)= & \sum_{l=0}^{L_{j}-2} \sum_{l^{\prime}=l+1}^{L_{j}-1} \widetilde{h}_{j, l} \widetilde{h}_{j, l^{\prime}} \gamma_{12}\left(t-l, t-l^{\prime}\right)=\sum_{l=0}^{L_{j}-2} \sum_{l^{\prime}=l+1}^{L_{j}-1} \widetilde{h}_{j, l} \widetilde{h}_{j, l^{\prime}} \gamma_{12}(\underbrace{l^{\prime}-l}_{=k>0})= \\
= & \sum_{l=0}^{L_{j}-2} \sum_{l^{\prime}=l+1}^{L_{j}-1} \widetilde{h}_{j, l} \widetilde{h}_{j, l^{\prime}} \frac{a b^{\left(l^{\prime}-l\right)-1}}{1-b^{2}}=\frac{a}{1-b^{2}} \sum_{l=0}^{L_{j}-2} \sum_{l^{\prime}=l+1}^{L_{j}-1} \widetilde{h}_{j, l} \widetilde{h}_{j, l^{\prime}} b^{\left(l^{\prime}-l-1\right)} .
\end{aligned}
$$

Notice that $F_{12, t}\left(\tau_{j}\right)$ and $B_{12, t}\left(\tau_{j}\right)$ do not depend on t. Figure 3.1 shows these two quantities for a time series with $\mathrm{T}=512$ calculated for the levels 0 to 9 . Also, $B_{12, t}\left(\tau_{j}\right)$ varies from -0.3 to 0.85 whereas $F_{12, t}\left(\tau_{j}\right)$ has its values close to zero, which is expected since $X_{1, t}$ depends on $X_{2, t-1}$. Figure 3.2 shows the average curves for $F_{12, t}\left(\tau_{j}\right)$ and $B_{12, t}\left(\tau_{j}\right)$ using 100 simulated time series of the process (3.9) considering $a=0.6$ and $b=0.3$ and the theoretical values are plotted together. Notice the values are very similar except for the beginning and the end of each scale, where the estimators lose precision due to the lack of the circular convolution terms. That imprecision is also perceptible in the empirical distribution of $F_{12, t}\left(\tau_{j}\right)$ and $B_{12, t}\left(\tau_{j}\right)$, presented in Appendix A. 


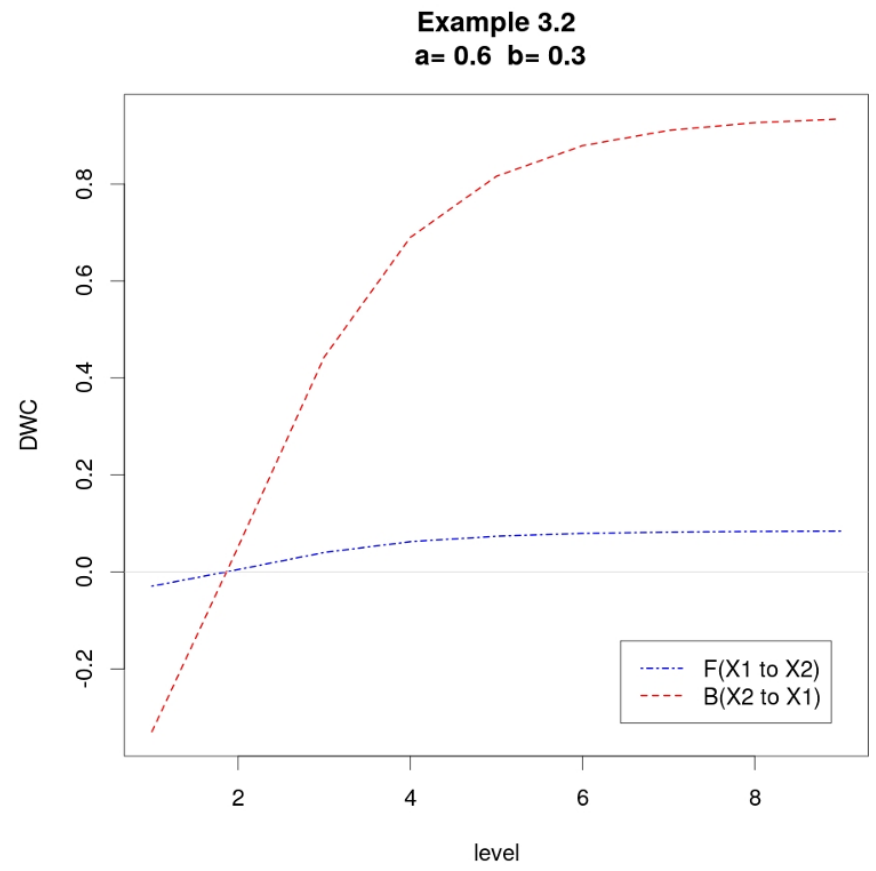

Figure 3.1. Example 3.2. Theoretical values for $F_{12, t}\left(\tau_{j}\right)$ and $B_{12, t}\left(\tau_{j}\right)$ for each level.
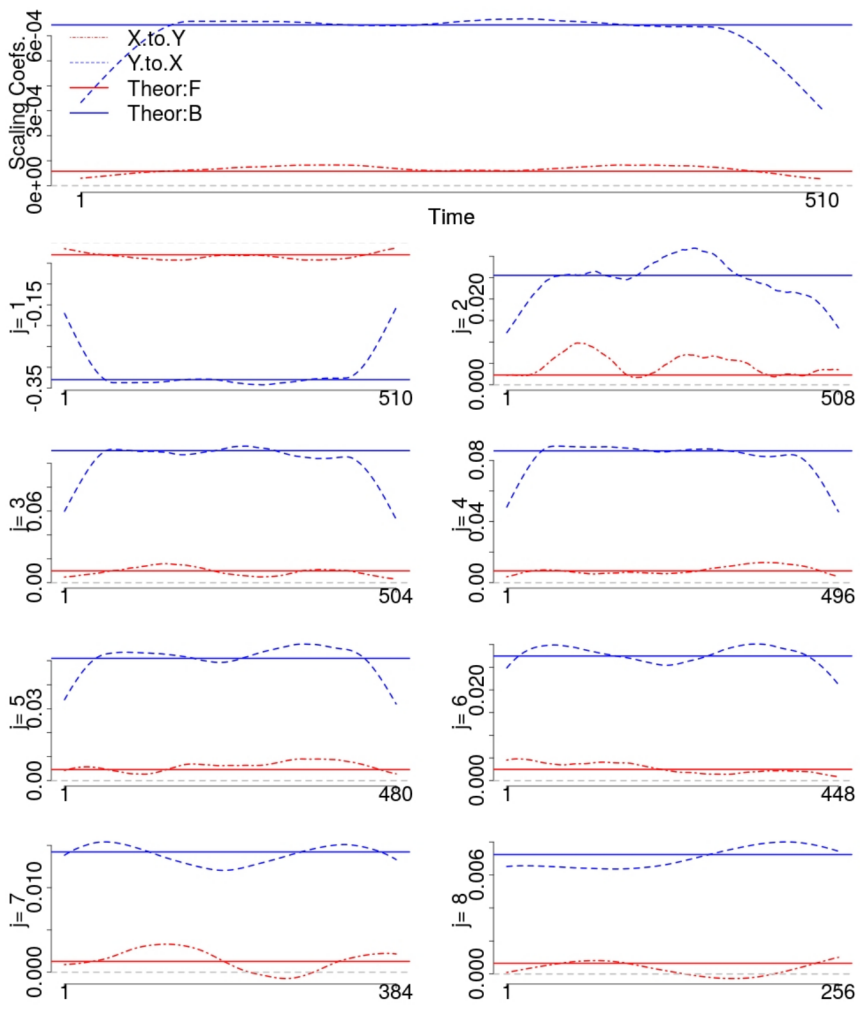

Figure 3.2. Example 3.2. Average $F_{12, t}\left(\tau_{j}\right)$ and $B_{12, t}\left(\tau_{j}\right)$ for one-hundred simulated time series of the process (3.9) using $a=0.6$ and $b=0.3$, and theoretical values for $F_{12, t}\left(\tau_{j}\right)$ and $B_{12, t}\left(\tau_{j}\right)$ for each level. 
Example 3.3 (VAR(1) with dependency in both directions). Consider now a bivariate VAR(1) with both direction's dependency, i.e., we represent the process as:

$$
\begin{gathered}
\mathbf{X}_{t}=\mathbf{A X}_{t-1}+\boldsymbol{\epsilon}(t) \\
\left(\begin{array}{l}
X_{1, t} \\
X_{2, t}
\end{array}\right)=\mathbf{A}\left(\begin{array}{l}
X_{1, t-1} \\
X_{2, t-1}
\end{array}\right)+\left(\begin{array}{l}
\epsilon_{1, t} \\
\epsilon_{2, t}
\end{array}\right), \\
\mathbf{A}=\left(\begin{array}{ll}
a_{11} & a_{12} \\
a_{21} & a_{22}
\end{array}\right)=\left(\begin{array}{ll}
0 & a \\
b & 0
\end{array}\right),
\end{gathered}
$$

$\boldsymbol{\epsilon}(t), a$ and $b$ as in Example 3.1.

In this example we have the past of $X_{2, t}$ affecting present observations of $X_{1, t}$ and vice-versa. $\Gamma(k)$ is calculated as follows:

$$
\begin{aligned}
\operatorname{vec}(\Gamma(0)) & =\left(I_{k}^{2}-\mathbf{A} \otimes \mathbf{A}\right)^{-1} \operatorname{vec}(\Sigma)=\left(\begin{array}{cccc}
\frac{1}{1-(a b)^{2}} & 0 & 0 & \frac{a^{2}}{1-(a b)^{2}} \\
0 & \frac{1}{1-(a b)^{2}} & \frac{a b}{1-(a b)^{2}} & 0 \\
0 & \frac{a b}{1-(a b)^{2}} & \frac{1}{1-(a b)^{2}} & 0 \\
\frac{b^{2}}{1-(a b)^{2}} & 0 & 0 & \frac{1}{1-(a b)^{2}}
\end{array}\right) \\
& =\left(\begin{array}{lllll}
\frac{1+a^{2}}{1-(a b)^{2}}, & 0, & 0, & \frac{1+b^{2}}{1-(a b)^{2}}
\end{array}\right) .
\end{aligned}
$$

Thus,

$$
\begin{aligned}
& \Gamma(0)=\left(\begin{array}{cc}
1+\frac{1+a^{2}}{1-(a b)^{2}} & 0 \\
0 & \frac{1+b^{2}}{1-(a b)^{2}}
\end{array}\right)=\left(\begin{array}{cc}
\gamma_{11}(0) & 0 \\
0 & \gamma_{22}(0)
\end{array}\right) \\
& \Gamma(1)=A \cdot \Gamma(0)=\left(\begin{array}{ll}
0 & a \\
b & 0
\end{array}\right) \cdot\left(\begin{array}{cc}
\gamma_{22}(0) & 0 \\
0 & \gamma_{11}(0)
\end{array}\right)=\left(\begin{array}{cc}
0 & a \gamma_{22}(0) \\
b \gamma_{11}(0) & 0
\end{array}\right) \\
& \Gamma(2)=A \cdot \Gamma(1)=\left(\begin{array}{ll}
0 & a \\
b & 0
\end{array}\right) \cdot\left(\begin{array}{cc}
0 & a \gamma_{22}(0) \\
b \gamma_{11}(0) & 0
\end{array}\right)=\left(\begin{array}{cc}
a b \gamma_{11}(0) & 0 \\
0 & a b \gamma_{22}(0)
\end{array}\right) \\
& \Gamma(3)=A \cdot \Gamma(2)=\left(\begin{array}{ll}
0 & a \\
b & 0
\end{array}\right) \cdot\left(\begin{array}{cc}
a b \gamma_{11}(0) & 0 \\
0 & a b \gamma_{22}(0)
\end{array}\right)=\left(\begin{array}{cc}
0 & a^{2} b \gamma_{22}(0) \\
0 b^{2} \gamma_{11}(0) & 0
\end{array}\right) \\
& \Gamma(4)=A \cdot \Gamma(3)=\left(\begin{array}{ll}
0 & a \\
b & 0
\end{array}\right) \cdot\left(\begin{array}{cc}
a b^{2} b \gamma_{22}(0) \\
a b^{2} \gamma_{11}(0) & 0
\end{array}\right)=\left(\begin{array}{cc}
a^{2} \gamma_{11}(0) & 0 \\
0 & a^{2} b^{2} \gamma_{22}(0)
\end{array}\right)
\end{aligned}
$$

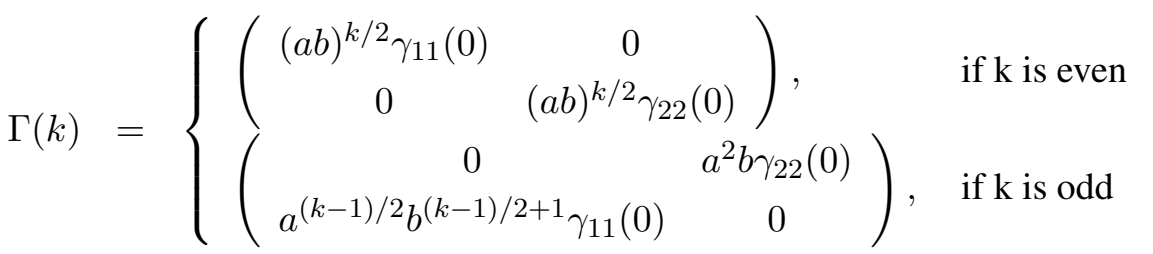


Since $\gamma_{11}(0)=\frac{1+a^{2}}{1-(a b)^{2}}$ and $\gamma_{22}(0)=\frac{1+b^{2}}{1-(a b)^{2}}$ then

$$
\gamma_{12}(k)= \begin{cases}\left(\frac{1+b^{2}}{1-(a b)^{2}}\right) a^{(k-1) / 2+1} b^{(k-1) / 2}, & \text { if } k>0 \text { and } \mathrm{k} \text { is odd } \\ \left.\frac{1+a^{2}}{1-(a b)^{2}}\right) a^{(1-k) / 2} b^{(1-k) / 2+1}, & \text { if } k<0 \text { and } \mathrm{k} \text { is odd }\end{cases}
$$

For $F_{12, t}\left(\tau_{j}\right)$ and $B_{12, t}\left(\tau_{j}\right)$ we have:

$$
\begin{aligned}
F_{12, t}\left(\tau_{j}\right) & =\sum_{l=1}^{L_{j}-1} \sum_{l^{\prime}=0}^{l-1} \widetilde{h}_{j, l} \widetilde{h}_{j, l^{\prime}} \gamma_{12}\left(t-l, t-l^{\prime}\right)=\sum_{l=1}^{L_{j}-1} \sum_{l^{\prime}=0}^{l-1} \widetilde{h}_{j, l} \widetilde{h}_{j, l^{\prime}} \mathbb{E}\left(X_{1, t-l} X_{2, t-l^{\prime}}\right)= \\
& =\sum_{l=1}^{L_{j}-1} \sum_{l^{\prime}=0}^{l-1} \widetilde{h}_{j, l} \widetilde{h}_{j, l^{\prime}} \gamma_{12}\left(t-l-\left(t-l^{\prime}\right)\right)=\sum_{l=1}^{L_{j}-1} \sum_{l^{\prime}=0}^{l-1} \widetilde{h}_{j, l} \widetilde{h}_{j, l^{\prime}} \gamma_{12}(\underbrace{l^{\prime}-l}_{=k<0})= \\
& =\sum_{l=1}^{L_{j}-1} \sum_{l^{\prime}=0}^{l-1} \widetilde{h}_{j, l} \widetilde{h}_{j, l^{\prime}}\left(\frac{1+a^{2}}{1-(a b)^{2}}\right) a^{\left(1-\left(l^{\prime}-l\right)\right) / 2} b^{\left(1-l^{\prime}-l\right) / 2+1} \mathbb{I}\left(l^{\prime}-l \text { is odd }\right)= \\
& =\frac{1+a^{2}}{1-(a b)^{2}} \sum_{l=1}^{L_{j}-1} \sum_{l^{\prime}=0}^{l-1} \widetilde{h}_{j, l} \widetilde{h}_{j, l^{\prime}} a^{\left(l-l^{\prime}+1\right) / 2} b^{1+\left(l-l^{\prime}+1\right) / 2} \mathbb{I}\left(l^{\prime}-l \text { is odd }\right) \\
B_{12, t}\left(\tau_{j}\right) & =\sum_{l=0}^{L_{j}-2} \sum_{l^{\prime}=l+1}^{L_{j}-1} \widetilde{h}_{j, l} \widetilde{h}_{j, l^{\prime}} \gamma_{12}\left(t-l, t-l^{\prime}\right)=\sum_{l=0}^{L_{j}-2} \sum_{l^{\prime}=l+1}^{L_{j}-1} \widetilde{h}_{j, l} \widetilde{h}_{j, l^{\prime}} \gamma_{12}(\underbrace{l^{\prime}-l}_{=k>0})= \\
= & \sum_{l=0}^{L_{j}-2} \sum_{l^{\prime}=l+1}^{L_{j}-1} \widetilde{h}_{j, l} \widetilde{h}_{j, l^{\prime}} a^{\left(l^{\prime}-l-1\right) / 2+1} b^{\left(l^{\prime}-l-1\right) / 2} \mathbb{I}\left(l^{\prime}-l \text { is odd }\right) \\
= & \frac{1+b^{2}}{1-(a b)^{2}} \sum_{l=0}^{L_{j}-2} \sum_{l^{\prime}=l+1}^{L_{j}-1} \widetilde{h}_{j, l} \widetilde{h}_{j, l^{\prime}} a^{\left(l^{\prime}-l-1\right) / 2+1} b^{\left(l^{\prime}-l-1\right) / 2} \mathbb{I}\left(l^{\prime}-l \text { is odd }\right) .
\end{aligned}
$$

Now, in this example, if $a=b$ then we have $F_{12, t}\left(\tau_{j}\right)=B_{12, t}\left(\tau_{j}\right)$. Figure 3.3 shows theoretical values of $F_{12, t}\left(\tau_{j}\right)$ and $B_{12, t}\left(\tau_{j}\right)$ with $\mathrm{T}=512$ for the levels 0 to 9 in two situations: $\mathrm{a}=\mathrm{b}=0.6$ and $\mathrm{a}=0.6$ and $\mathrm{b}=0.3$.

Figure 3.4 and Figure 3.5 show the average curve for $F_{12, t}\left(\tau_{j}\right)$ and $B_{12, t}\left(\tau_{j}\right)$ using 100 simulated time series of the process (3.10) considering $(a, b)=(0.6,0.6)$ and $(a, b)=(0.6,0.3)$, respectively. The theoretical values of $F_{12, t}\left(\tau_{j}\right)$ and $B_{12, t}\left(\tau_{j}\right)$ are also plotted on the graph. Again, the average values are very similar to the theoretical values and the same imprecision happens on the board of the scales. The empirical distribution of both quantities in both examples is presented in Appendix A.

Example 3.4 (Vector Moving Average Model - VMA(1)). In this example we consider a Vector Moving Average Process of order 1, given by:

$$
\begin{gathered}
\mathbf{X}_{t}=\Theta \epsilon_{t-1}+\boldsymbol{\epsilon}(t) \\
\left(\begin{array}{c}
X_{1, t} \\
X_{2, t}
\end{array}\right)=\left(\begin{array}{ll}
\theta_{11} & \theta_{12} \\
\theta_{21} & \theta_{22}
\end{array}\right)\left(\begin{array}{c}
\epsilon_{1, t-1} \\
\epsilon_{2, t-1}
\end{array}\right)+\left(\begin{array}{c}
\epsilon_{1, t} \\
\epsilon_{2, t}
\end{array}\right),
\end{gathered}
$$



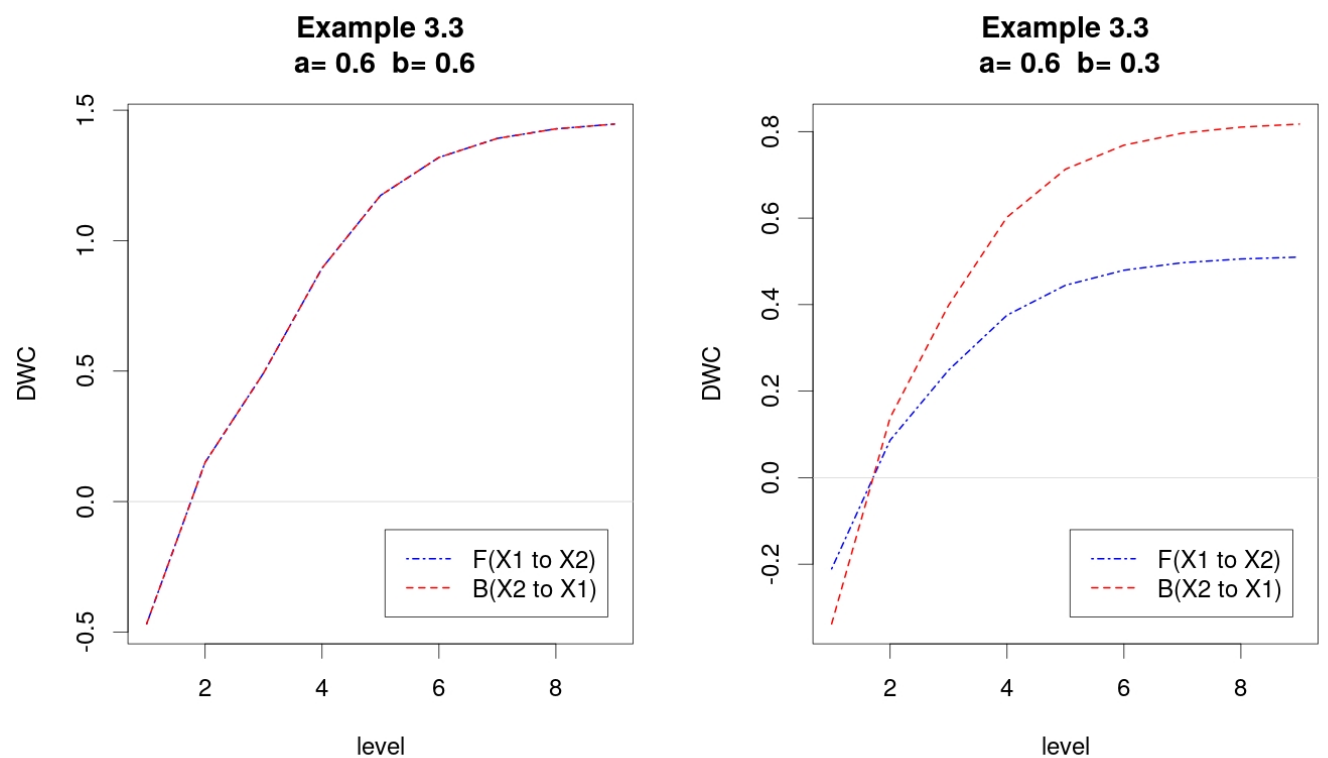

Figure 3.3. Example 3.3. Theoretical values for $F_{12, t}\left(\tau_{j}\right)$ and $B_{12, t}\left(\tau_{j}\right)$ for each level.

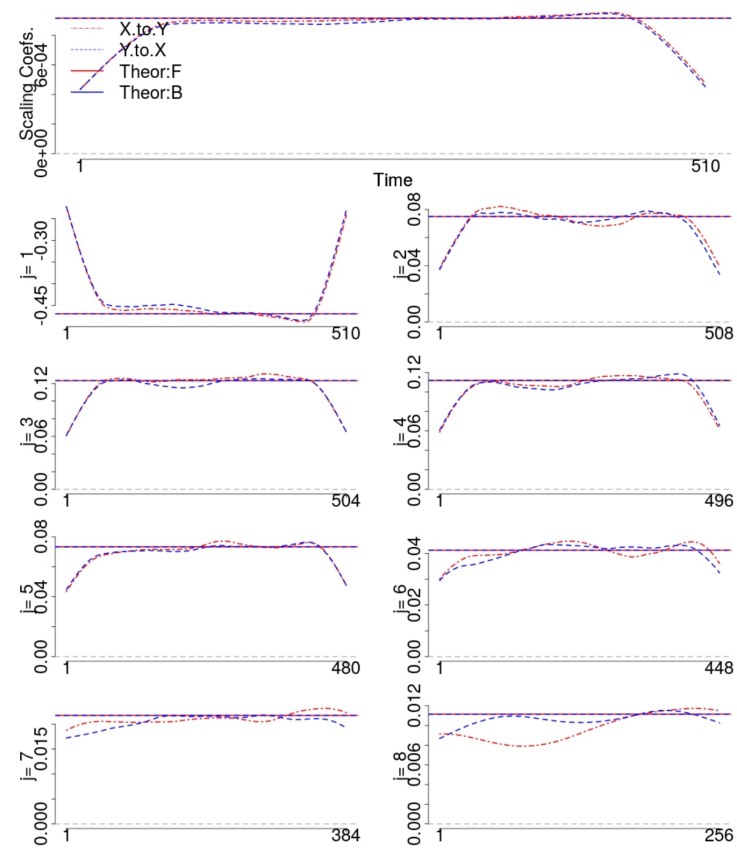

Figure 3.4. Example 3.3. Average $F_{12, t}\left(\tau_{j}\right)$ and $B_{12, t}\left(\tau_{j}\right)$ for 100 simulated time series of the process (3.10) using $a=b=0.6$ and theoretical values for $F_{12, t}\left(\tau_{j}\right)$ and $B_{12, t}\left(\tau_{j}\right)$ for each level.

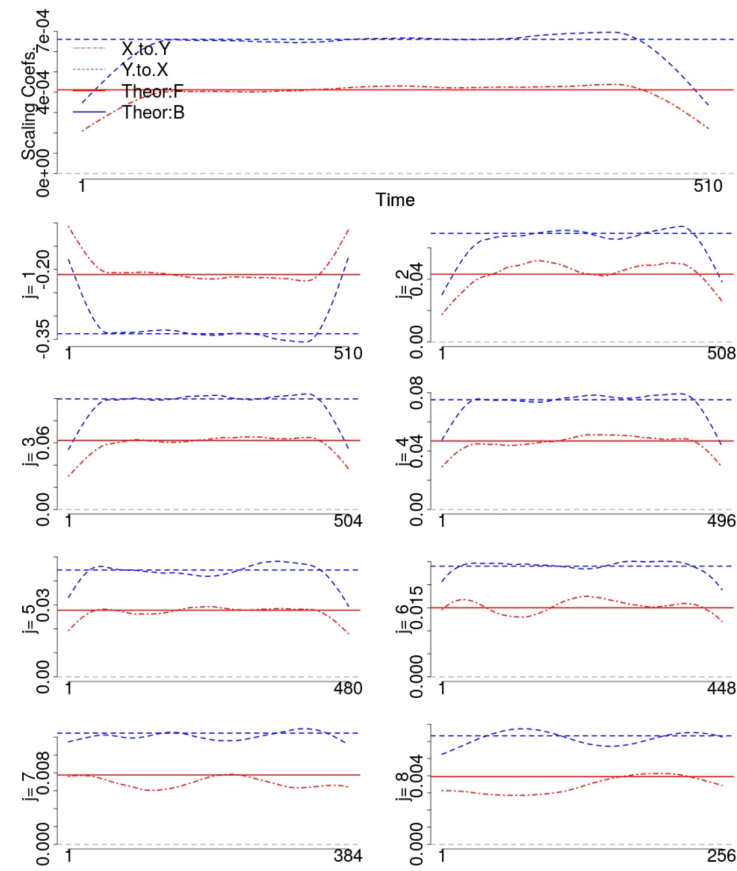

Figure 3.5. Example 3.3. Average $F_{12, t}\left(\tau_{j}\right)$ and $B_{12, t}\left(\tau_{j}\right)$ for 100 simulated time series of the process (3.10) using $a=0.6$ and $b=0.3$, and theoretical values for $F_{12, t}\left(\tau_{j}\right)$ and $B_{12, t}\left(\tau_{j}\right)$ for each level. 
$\boldsymbol{\epsilon}(t), a$ and $b$ as in Example 3.1.

$\Gamma(k)$ is calculated as follows:

$$
\Gamma(k)=\mathbb{E}\left(\mathbf{X}_{t} \mathbf{X}_{t-k}^{\top}\right)=\mathbb{E}\left[\left(\begin{array}{c}
X_{1, t} \\
X_{2, t}
\end{array}\right)\left(\begin{array}{ll}
X_{1, t} & X_{2, t}
\end{array}\right)\right]=\left(\begin{array}{cc}
\gamma_{11}(k) & \gamma_{12}(k) \\
\gamma_{21}(k) & \gamma_{22}(k)
\end{array}\right)
$$

Now, for each of the components we have:

$$
\begin{aligned}
\gamma_{11}(k) & =\mathbb{E}\left(\epsilon_{1, t} \epsilon_{1, t-k}\right)-\theta_{11} \mathbb{E}\left(\epsilon_{1, t} \epsilon_{1, t-k-1}\right)-\theta_{11} \mathbb{E}\left(\epsilon_{1, t-1} \epsilon_{1, t-k}\right)+\theta_{11}^{2} \mathbb{E}\left(\epsilon_{1, t-1} \epsilon_{1, t-k-1}\right) \\
\gamma_{22}(k) & =\mathbb{E}\left(\epsilon_{2, t} \epsilon_{2, t-k}\right)-\theta_{22} \mathbb{E}\left(\epsilon_{2, t} \epsilon_{2, t-k-1}\right)-\theta_{22} \mathbb{E}\left(\epsilon_{2, t-1} \epsilon_{2, t-k}\right)+\theta_{22}^{2} \mathbb{E}\left(\epsilon_{2, t-1} \epsilon_{2, t-k-1}\right) \\
\gamma_{12}(k) & =\mathbb{E}\left[\left(\theta_{11} \epsilon_{1, t-1}+\theta_{12} \epsilon_{2, t-1}+\epsilon_{1, t}\right)\left(\theta_{21} \epsilon_{1, t-k-1}+\theta_{22} \epsilon_{2, t-k-1}+\epsilon_{2, t-k}\right)\right]= \\
& =\theta_{11} \theta_{21} \mathbb{E}\left(\epsilon_{1, t-1} \epsilon_{1, t-k-1}\right)+\theta_{12} \theta_{22} \mathbb{E}\left(\epsilon_{2, t-1} \epsilon_{2, t-k-1}\right)+\theta_{12} \mathbb{E}\left(\epsilon_{2, t-1} \epsilon_{2, t-k}\right)+\theta_{21} \mathbb{E}\left(\epsilon_{1, t} \epsilon_{1, t-k-1}\right)
\end{aligned}
$$

Since $\gamma_{21}(k)=\gamma_{12}(-k)$, we have:

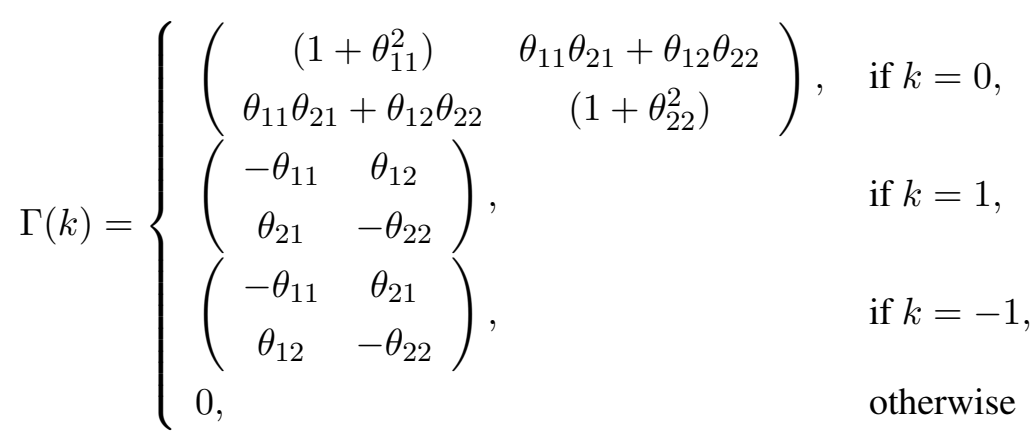

Finally we can calculate $F_{12, t}\left(\tau_{j}\right)$ and $B_{12, t}\left(\tau_{j}\right)$ for this case. For instance, considering $\theta_{11}=0$, then we have:

$$
\begin{aligned}
F_{12, t}\left(\tau_{j}\right) & =\sum_{l=1}^{L_{j}-1} \sum_{l^{\prime}=1}^{l-1} \widetilde{h}_{j, l} \widetilde{h}_{j, l^{\prime}} \gamma_{12}\left(t-l, t-l^{\prime}\right)=\sum_{l=1}^{L_{j}-1} \sum_{l^{\prime}=1}^{l-1} \widetilde{h}_{j, l} \widetilde{h}_{j, l} \gamma_{12}(\underbrace{l^{\prime}-l}_{\neq 0 \text { if } l^{\prime}=l-1})= \\
& =\sum_{l=1}^{L_{j}-1} \widetilde{h}_{j, l} \widetilde{h}_{l-1} \gamma_{12}(-1)=\theta_{21} \sum_{l=1}^{L_{j}-1} \widetilde{h}_{j, l} \widetilde{h}_{l-1}, \\
B_{12, t}\left(\tau_{j}\right)= & \sum_{l=0}^{L_{j}-2} \sum_{l^{\prime}=l+1}^{L_{j}-1} \widetilde{h}_{j, l} \widetilde{h}_{j, l^{\prime}} \gamma_{12}\left(t-l, t-l^{\prime}\right)=\sum_{l=0}^{L_{j}-2} \sum_{l^{\prime}=l+1}^{L_{j}-1} \widetilde{h}_{j, l} \widetilde{h}_{j, l} \gamma_{12}(\underbrace{l^{\prime}-l}_{\neq 0 \text { if } l^{\prime}=l+1})= \\
= & \sum_{l=0}^{L_{j}-2} \widetilde{h}_{j, l} \widetilde{h}_{l+1} \gamma_{12}(1)=\theta_{12} \sum_{l=0}^{L_{j}-2} \widetilde{h}_{j, l} \widetilde{h}_{l+1}=\theta_{12} \sum_{l^{*}=1}^{L_{j}-1} \widetilde{h}_{l^{*}-1} \widetilde{h}_{l^{*}} .
\end{aligned}
$$

We see that in this case, both $F_{12, t}\left(\tau_{j}\right)$ and $B_{12, t}\left(\tau_{j}\right)$ depend only on $\theta_{12}$ and $\theta_{21}$ (see Figure 3.6).

Figure 3.7 shows the average curve for $F_{12, t}\left(\tau_{j}\right)$ and $B_{12, t}\left(\tau_{j}\right)$ using 100 simulated time series of the process (3.4) considering $\theta_{12}=0.6$ and $\theta_{21}=0.3$ with the theoretical values plotted together. Notice the values are very similar except for the beginning and the end of each scale, where the estimators lose precision due to the lack of the circular convolution terms. Empirical distributions for these two 
quantities considering the 100 simulated time series are also presented in Appendix A.

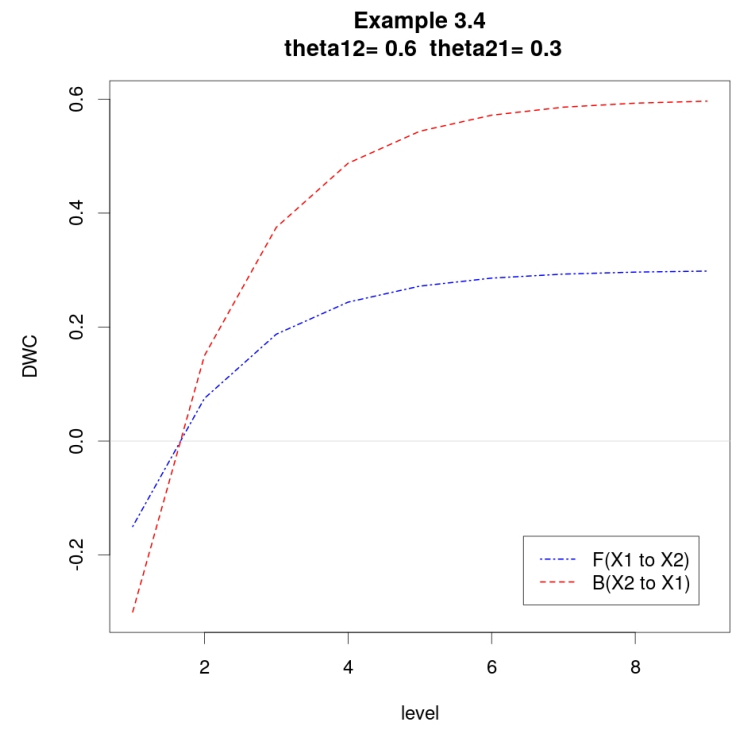

Figure 3.6. Example 3.4. Theoretical values for $F_{12, t}\left(\tau_{j}\right)$ and $B_{12, t}\left(\tau_{j}\right)$ for each level.
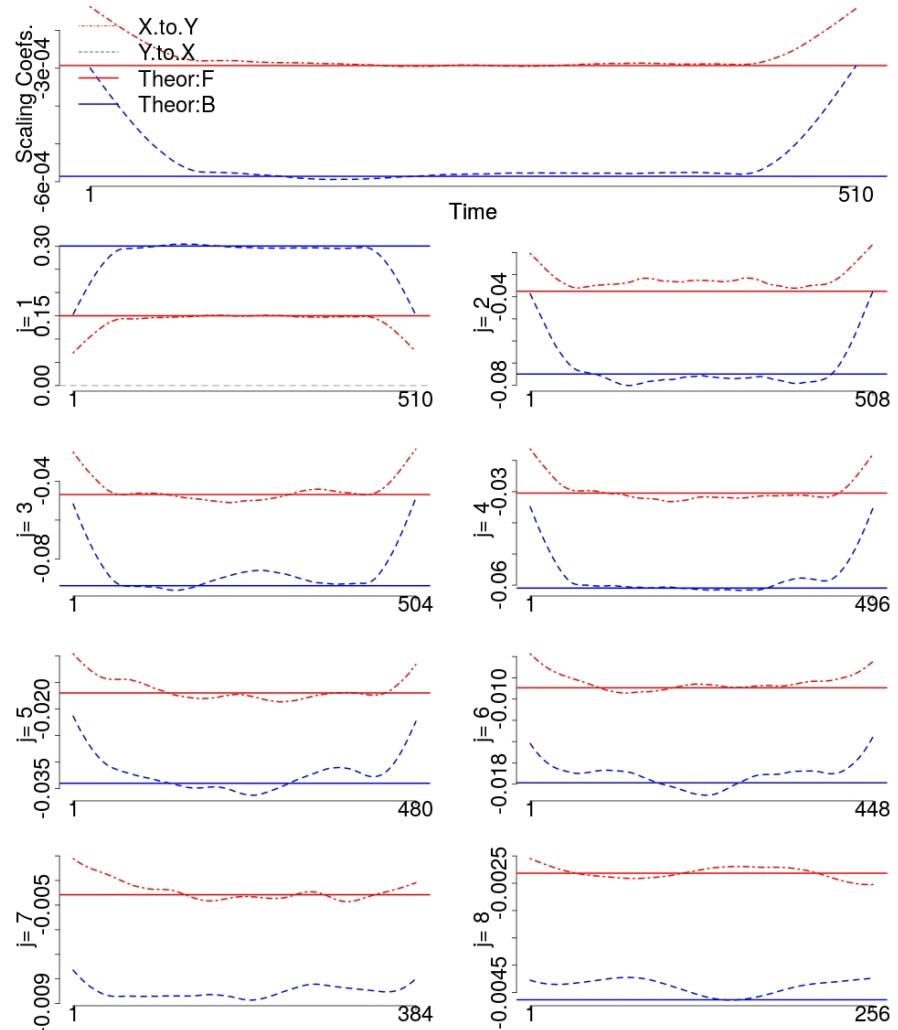

Figure 3.7. Example 3.4. Average $F_{12, t}\left(\tau_{j}\right)$ and $B_{12, t}\left(\tau_{j}\right)$ for 100 simulated time series of the process (3.4) using $\theta_{12}=0.6$ and $\theta_{21}=0.3$, and theoretical values for $F_{12, t}\left(\tau_{j}\right)$ and $B_{12, t}\left(\tau_{j}\right)$ for each level. 


\section{Chapter 4}

\section{Partial Directed Wavelet Covariance}

In this chapter we present other two contributions of the study. We propose two different approaches to calculate the Partial Directed Wavelet Covariance (pDWC). The main idea behind the pDWC is to calculate the relation between two components of a multivariate process $\mathbf{X}_{t}$ after removing the effect the other components. The first approach is based on a linear filter while the second considers that the process is a time-varying VAR of order $p: \operatorname{tv}-\operatorname{VAR}(\mathrm{p})$. This chapter is organized as follows: we first present on Section 4.1 the pDWC with a linear filter and its properties, followed by a simulation to evaluate its effectiveness. In Section 4.2 we present the second approach, based on tv-VAR(p) models. Next we present its estimators, properties and simulations. We will abbreviate the first proposal merely as $p D W C$ and the second as $p p D W C$ in order to differentiate them along the text. A comparison between these two methodologies with DWC, PDC and WTC will be presented in Chapter 5.

\section{1 pDWC with a linear filter: pDWC}

Recall from Theorem 2.1 that if we have an N-variate process $\mathbf{Z}_{t}=\left[\begin{array}{ll}\mathbf{Y}_{t} & \mathbf{X}_{t}\end{array}\right]_{(N \times 1)}^{\top}$ as in (2.7), with discrete time $t \in \mathbb{Z}$, mean and covariance matrices given by (2.8) and we want to calculate the covariance between two components of $\mathbf{Y}_{t}$, say $Y_{1, t}$ and $Y_{2, t}$, after removing the effect of the other components of $\mathbf{X}_{t}$, then $\epsilon_{t}$ in (2.13) is the residual process after filtering $\mathbf{Y}_{t}$ with the best linear filter based on $\mathbf{X}_{t} . \epsilon_{t}$ has covariance matrix given by (2.14) and is called the partial covariance of $Y_{1}$ and $Y_{2}$ after removing the linear influence of $\mathbf{X}_{t}$.

Definition 4.1. Given the following quantities:

- $\boldsymbol{\epsilon}_{t}$ in (2.13), the residual process after filtering $\mathbf{Y}_{t}$ with $\mathbf{X}_{t}$ and covariance matrix given by (2.14);

- $\widetilde{W}_{j, t}^{\epsilon_{k}}=\sum_{l=0}^{L_{j}-1} \widetilde{h}_{j, l} \epsilon_{k, t-l \operatorname{Mod} T}, j=1, \ldots, J, t=1, \ldots, T, k=1, \ldots, s$, the MODWT of the $k$-th component of $\epsilon_{t}$;

- $\nu_{\epsilon_{k}, t}^{2}\left(\tau_{j}\right) \equiv \operatorname{var}\left(\widetilde{W}_{j, t}^{\epsilon_{k}}\right)$, the variance of $\widetilde{W}_{j, t}^{\epsilon_{k}}$;

- $\nu_{(k, m), t}^{\epsilon}\left(\tau_{j}\right)=\operatorname{Cov}\left(\widetilde{W}_{j, t}^{\epsilon_{k}}, \widetilde{W}_{j, t}^{\epsilon_{m}}\right), k, m=1, \ldots, s, j=1, \ldots, J$ the wavelet covariance of $\epsilon_{k}$ with $\epsilon_{m}$. 
We define the Partial Directed Wavelet Covariance of $Y_{k}$ and $Y_{m}$ after removing the linear effects of $\mathbf{X}_{t}$ as :

$$
\begin{aligned}
p F_{(k, m), t}\left(\tau_{j}\right) & =\sum_{l=1}^{L_{j}-1} \sum_{l^{\prime}=0}^{l-1} \widetilde{h}_{j, l}\left(\widetilde{h}_{j, l^{\prime}}\right)^{H} \Sigma_{\epsilon_{k} \epsilon_{m}}\left(t-l, t-l^{\prime}\right), \\
p B_{(k, m), t}\left(\tau_{j}\right) & =\sum_{l=0}^{L_{j}-2} \sum_{l^{\prime}=l+1}^{L_{j}-1} \widetilde{h}_{j, l}\left(\widetilde{h}_{j, l^{\prime}}\right)^{H} \Sigma_{\epsilon_{k} \epsilon_{m}}\left(t-l, t-l^{\prime}\right) .
\end{aligned}
$$

with $p F_{(k, m), t}\left(\tau_{j}\right)$ representing the influence of $Y_{k}$ in $Y_{m}$ and $p B_{(k, m), t}\left(\tau_{j}\right)$ the influence of $Y_{m}$ in $Y_{k}$. $\Sigma_{\epsilon_{k} \epsilon_{m}}($.$) is the (k,m)-th element of the matrix \Sigma_{\epsilon \epsilon}$ in (2.14), the cross covariance function between $\epsilon_{k}$ and $\epsilon_{m}$.

\section{2 pDWC assuming a tv-VAR(p) model: ppDWC}

Now suppose we have a $D$-dimension time-varying Vector Autoregressive $(t v-V A R)$ of order $p$ that fulfills the local stationarity assumptions in Definition 2.6 and is expressed by:

$$
\mathbf{X}_{t, T}=\boldsymbol{\mu}(u)+\sum_{k=1}^{p} \mathbf{A}_{k}(u)^{\top} \mathbf{X}_{t-k, T}+\boldsymbol{\epsilon}(t)
$$

with:

- $u=t / T, t=1, \ldots, T$;

- $\boldsymbol{\epsilon}(t)=\left(\epsilon_{1}(t), \ldots, \epsilon_{D}(t)\right)^{\top}$ i.i.d. with $\mathbb{E}(\boldsymbol{\epsilon}(t))=\underset{\sim}{0}, \mathbb{E}(|\boldsymbol{\epsilon}(t)|)<\infty$, and variance matrix $V(\boldsymbol{\epsilon}(t))=\Sigma(t)=\operatorname{diag}\{\boldsymbol{\sigma}(t)\}$, diagonal matrix of the vector $\boldsymbol{\sigma}(t)$;

- $\boldsymbol{\mu}(u)_{D \times 1}, \mathbf{A}_{1}(u)_{D \times D}, \ldots, \mathbf{A}_{p}(u)_{D \times D}$ and $\boldsymbol{\sigma}(t)_{D \times 1}$ have bounded variation for each one of their components, e.g. if $\mathbf{A}_{1}(u)$ is written as:

$$
\mathbf{A}_{1}(u)=\left(\begin{array}{cccc}
a_{11}^{(1)}(u) & a_{12}^{(1)}(u) & \ldots & a_{1, D}^{(1)}(u) \\
a_{21}^{(1)}(u) & a_{22}^{(1)}(u) & \ldots & a_{2, D}^{(1)}(u) \\
\vdots & & \ddots & \vdots \\
a_{D, 1}^{(1)}(u) & a_{D, 2}^{(1)}(u) & \ldots & a_{D, D}^{(1)}(u)
\end{array}\right)=\left(\begin{array}{c}
a_{1 \bullet}^{(1)}(u) \\
a_{2 \bullet}^{(1)}(u) \\
\vdots \\
a_{D \bullet}^{(1)}(u)
\end{array}\right)
$$

then each $a_{i j}^{(1)}(u), i=1, \ldots, D, j=1, \ldots, D$ is a function of bounded variation.

Proposition 4.1. Consider $\mathbf{X}_{t, T}$ a D-dimension time-varying Vector Autoregressive (tv-VAR) of order p expressed in (4.3) that satisfies the locally stationarity assumptions of Definition 2.6. Let $\left\{\widetilde{h}_{j, l}\right\}$ be the $j$-th level of the MODWT filter associated with scale $\tau_{j}$ with width $L_{j}=\left(2^{j}-1\right)\left(L_{1}-1\right)+1$ and $W_{j, t}^{(1)}$ and $W_{j, t}^{(2)}$ the MODWT of components $X_{t, T}^{(1)}$ and $X_{t, T}^{(2)}$ of $\mathbf{X}_{t, T}$. Then

$$
\begin{aligned}
\nu\left(X^{(1)}, X^{(2)}, \tau_{j}\right)= & \operatorname{Cov}\left(\widetilde{W}_{j, t}^{(1)}, \widetilde{W}_{j, t}^{(2)}\right)=\widetilde{W}_{j, t}\left(\mu_{1}(u)\right) \widetilde{W}_{j, t}\left(\mu_{2}(u)\right)+ \\
& +p p F_{X_{1}, X_{2}, t}\left(\tau_{j}\right)+p p B_{X_{1}, X_{2}, t}\left(\tau_{j}\right)+ \\
& +R\left(X_{1} \rightleftharpoons X_{2}, \tau_{j}, t\right)+R\left(X_{1} \leftrightarrow X_{1}, \tau_{j}, t\right)+R\left(X_{2} \leftrightarrow X_{2}, \tau_{j}, t\right)+
\end{aligned}
$$




$$
\begin{aligned}
& +R\left(X_{1} \leftrightarrow\left\{X_{3}, \ldots, X_{D}\right\}, \tau_{j}, t\right)+R\left(X_{2} \leftrightarrow\left\{X_{3}, \ldots, X_{D}\right\}, \tau_{j}, t\right)+ \\
& +R\left(\left\{X_{3}, \ldots, X_{D}\right\} \leftrightarrow\left\{X_{3}, \ldots, X_{D}\right\}, \tau_{j}, t\right)+ \\
& +\overline{\mathscr{A}} . \sigma\left(\frac{t-k}{T}\right)
\end{aligned}
$$

with $\overline{\mathscr{A}}$ as in (B.7), $R($.$) as in (B.9), ppF(.) and p p B($.$) as in (B.10) and (B.11), respectively.$

Proof. See Appendix B.

So, Proposition 4.1 tells us that the total energy between two signals from a D-dimensional $t v$-VAR process $\mathbf{X}_{t, T}$ can be written as a sum of the relations between these two components, their relations with the other components, the MODWT of the two processes' mean functions and a function of the error variability, $\overline{\mathscr{A}}$. Therefore, we will use $p p F($.$) and p p B($.$) to evaluate the influences between$ components $X_{t, T}^{(1)}$ and $X_{t, T}^{(2)}$ on each other, excluding the influences of the other components of $\mathbf{X}_{t, T}$.

Corollary 4.1.1. For $D=2$ :

$$
\begin{aligned}
\nu\left(X^{(1)}, X^{(2)}, \tau_{j}\right) & =\widetilde{W}_{j, t}\left(\mu_{1}(u)\right) \widetilde{W}_{j, t}\left(\mu_{2}(u)\right)+ \\
& +p p F_{X_{1}, X_{2}, t}\left(\tau_{j}\right)+p p B_{X_{1}, X_{2}, t}\left(\tau_{j}\right)+ \\
& +R\left(X_{1} \rightleftharpoons X_{1}\right)+R\left(X_{2} \rightleftharpoons X_{2}\right)+R\left(X_{1} \rightleftharpoons X_{2}\right)+ \\
& +\overline{\mathscr{A}} \cdot \sigma\left(\frac{t-k}{T}\right)
\end{aligned}
$$

For $D=3$ :

$$
\begin{aligned}
\nu\left(X^{(1)}, X^{(2)}, \tau_{j}\right) & =\widetilde{W}_{j, t}\left(\mu_{1}(u)\right) \widetilde{W}_{j, t}\left(\mu_{2}(u)\right)+ \\
& +p p F_{X_{1}, X_{2}, t}\left(\tau_{j}\right)+p p B_{X_{1}, X_{2}, t}\left(\tau_{j}\right)+ \\
& +R\left(X_{1} \rightleftharpoons X_{1}\right)+R\left(X_{2} \rightleftharpoons X_{2}\right)+R\left(X_{1} \rightleftharpoons X_{2}\right)+ \\
& +R\left(X_{1} \leftrightarrow X_{3}\right)+R\left(X_{2} \leftrightarrow X_{3}\right)+R\left(X_{3} \rightleftharpoons X_{3}\right)+ \\
& +\overline{\mathscr{A}} \cdot \sigma\left(\frac{t-k}{T}\right),
\end{aligned}
$$

As in Chapter 3, next examples will help us to understand how the estimators of pDWC and ppDWC behave in stationary situations. This will be important when we propose tests in Chapter 6 . Examples considering locally stationary processes will be presented in Chapter 5.

Example 4.1 (pDWC using a linear filter for VAR(1)). Consider the following model:

$$
\mathbf{X}_{t}=\mathbf{A X}_{t-1}+\boldsymbol{\epsilon}(t)=\left(\begin{array}{ccc}
0.0 & 0 & 0.6 \\
0.5 & 0 & 0.3 \\
0.0 & 0.3 & 0.3
\end{array}\right) \mathbf{X}_{t-1}+\boldsymbol{\epsilon}(t), t \in 1, \ldots, 1024
$$

with $\epsilon(t)$ as in (3.7). The relations between their components are illustrated in Figure 4.1. We expect a bidirectional relation between $X_{2, t}$ and $X_{3, t}$ and a unidirectional influence from $X_{3, t}$ to $X_{1, t}$ and from $X_{1, t}$ to $X_{2, t}$. Figures 4.2 to 4.4 present the average pDWC estimated by 100 replications of the 
process (4.4) using $\mathrm{T}=1024$. We can see the scaling and wavelet coefficients capture the simulated impact when the relation is unidirectional, as in $X_{1} \rightarrow X_{2}$ and $X_{3} \rightarrow X_{1}$. They have absolute values according to the simulation. However, the bidirectional relation $X_{2} \leftrightarrow X_{3}$ appears only in levels 4 and 9 of the wavelet coefficients.

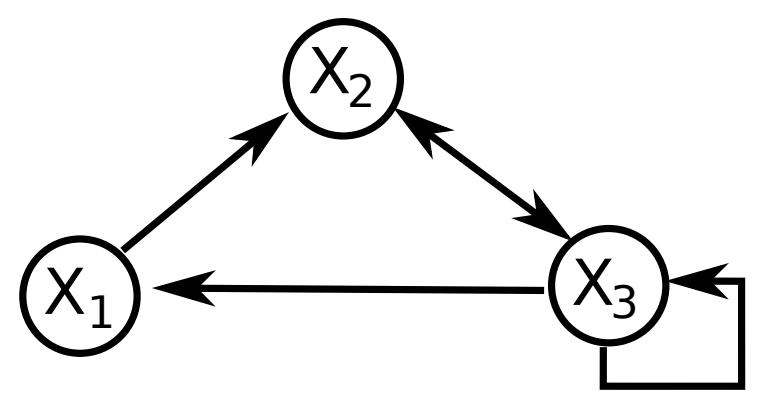

Figure 4.1. Relations between components of the process (4.4)

Notice that the pDWC of $Y_{k}$ and $Y_{m}$ after removing the linear effects of $\mathbf{X}_{t}$ (4.1 and 4.2), are in fact the DWC between $\epsilon_{k}$ and $\epsilon_{m}$. Therefore, we propose the estimators of $p F_{(k, m), t}\left(\tau_{j}\right)$ and $p B_{(k, m), t}\left(\tau_{j}\right)$ as in (3.4 and 3.5):

$$
\begin{gathered}
\widehat{p F}_{(k, m), t}\left(\tau_{j}\right)=\sum_{l=1}^{L_{j}-1} \sum_{l^{\prime}=0}^{l-1} \widetilde{h}_{j, l}\left(\tilde{h}_{j, l^{\prime}}\right)^{H}\left[\hat{c}_{\epsilon_{m}, \epsilon_{k}}\left(\frac{(t-l)}{T},\left(l^{\prime}-l\right)\right)\right], \\
\widehat{p B}_{(k, m), t}\left(\tau_{j}\right)=\sum_{l=0}^{L_{j}-2} \sum_{l^{\prime}=l+1}^{L_{j}-1} \widetilde{h}_{j, l}\left(\tilde{h}_{j, l^{\prime}}\right)^{H}\left[\hat{c}_{\epsilon_{m}, \epsilon_{k}}\left(\frac{(t-l)}{T},\left(l^{\prime}-l\right)\right)\right] .
\end{gathered}
$$

It follows that Propositions 3.2 and 3.3 are also valid for these estimators. This means there is a bias related to the non-stationarity of the data, but the estimators (4.6) and (4.6) are asymptotically consistent.

The following examples also consider stationary processes and their main purpose is to better understand how the estimators of ppDWC behave in situations where we can calculate their theoretical values.

Example 4.2 (ppDWC for Bivariate stationary VAR(1)). Consider $\mathbf{X}_{t}=\left(\begin{array}{ll}X_{t}^{(1)} & X_{t}^{(2)}\end{array}\right)^{\top}$ a stationary bivariate $\operatorname{VAR}(1)$ and define the sets $A=\left\{\left(l, m, l^{\prime}, m^{\prime}\right) \mid l+m>l^{\prime}+m^{\prime}\right.$ and $\left(l+m, l^{\prime}+m^{\prime}\right) \in$ $[0, t] \times[0, t]\}$ and $B=\left\{\left(l, m, l^{\prime}, m^{\prime}\right) \mid l+m<l^{\prime}+m^{\prime}\right.$ and $\left.\left(l+m, l^{\prime}+m^{\prime}\right) \in[0, t] \times[0, t]\right\}$ as illustrated in Figure B.1. The ppDWC for this process is given in Corollary 4.1.1, i.e.:

$$
\begin{aligned}
p p F_{X_{1}, X_{2}, t}\left(\tau_{j}\right) & =\sum_{A} \widetilde{h}_{j, l} \widetilde{h}_{j, l^{\prime}} a_{1,1}^{(m)}(u) a_{2,2}^{\left(m^{\prime}\right)}(u) \gamma_{X_{1}, X_{2}}\left(t-l-m, t-l^{\prime}-m^{\prime}\right)+ \\
& +\sum_{B} \widetilde{h}_{j, l} \widetilde{h}_{j, l^{\prime}} a_{1,2}^{(m)}(u) a_{2,1}^{\left(m^{\prime}\right)}(u) \gamma_{X_{2}, X_{1}}\left(t-l-m, t-l^{\prime}-m^{\prime}\right),
\end{aligned}
$$



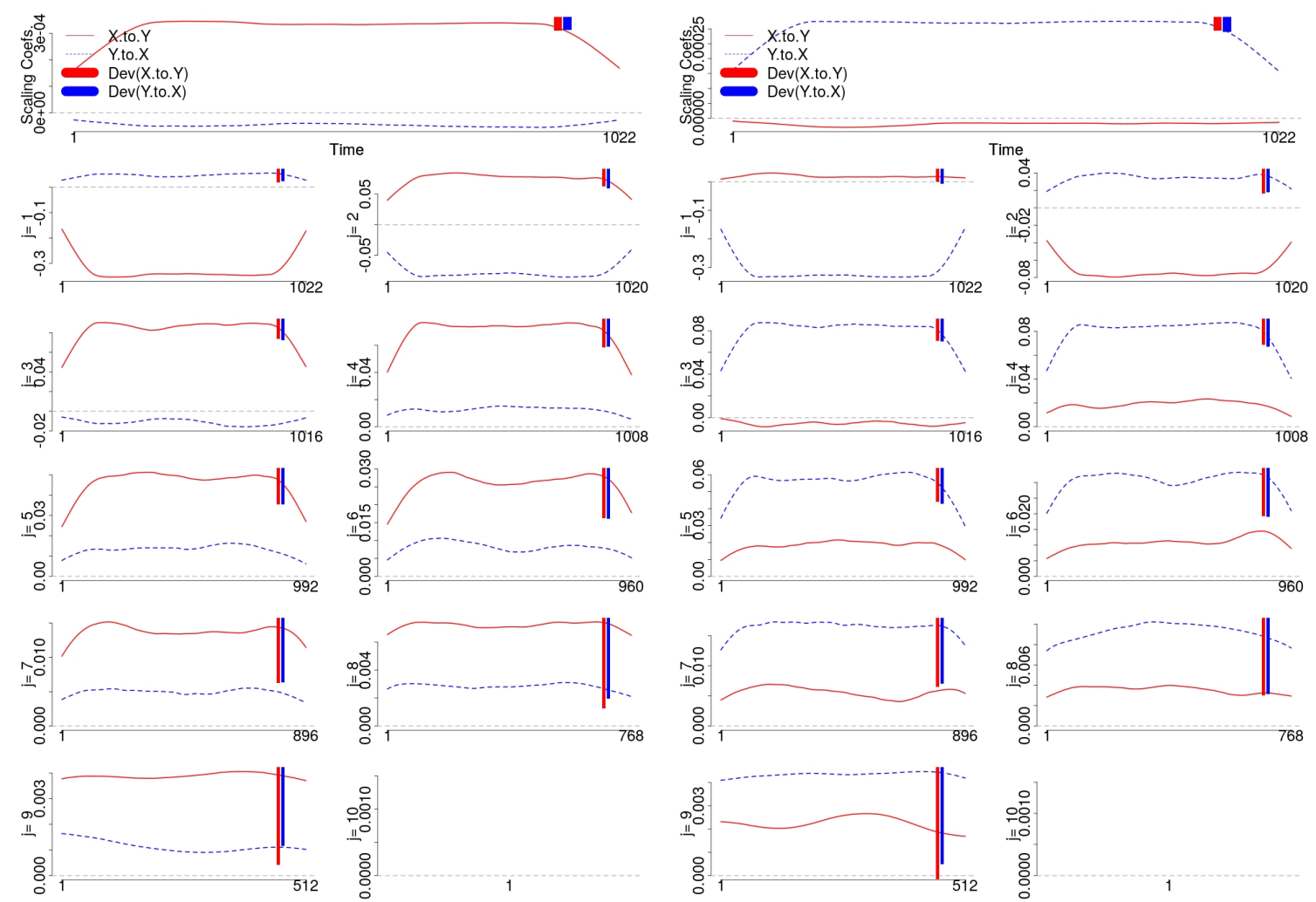

Figure 4.2. Relations between components $X_{1, t}$ and Figure 4.3. Relations between components $X_{1, t}$ and $X_{2, t}$ in process (4.4) estimated by $p D W C$. $X_{3, t}$ in process (4.4) estimated by $\mathrm{pDWC}$.

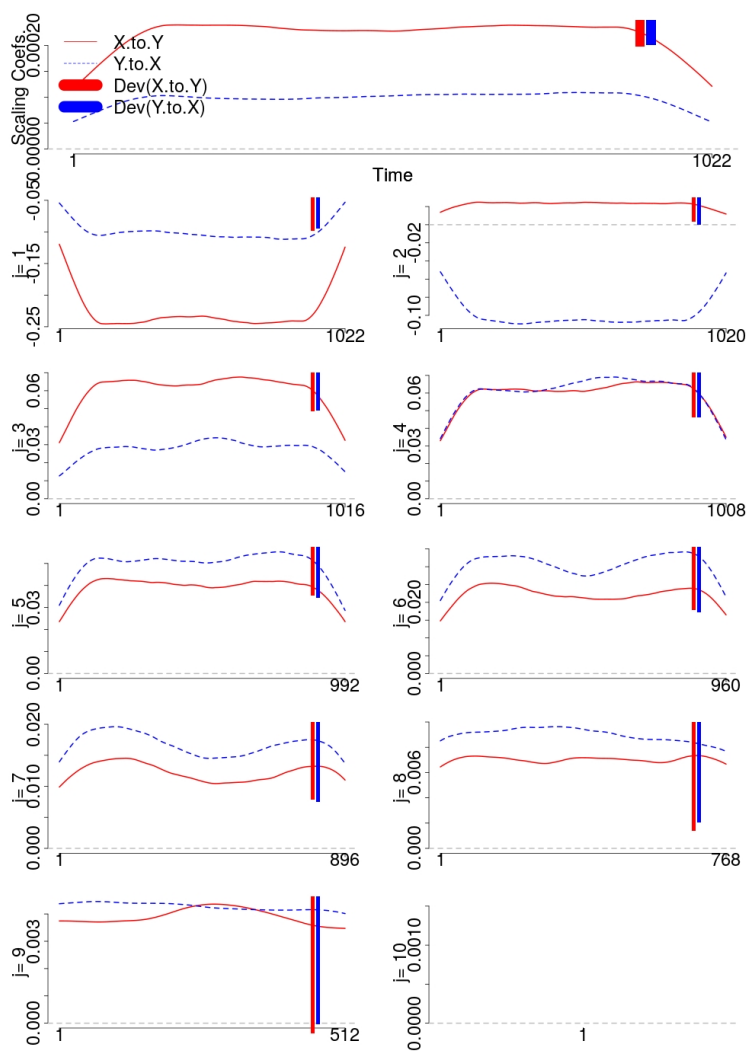

Figure 4.4. Relations between components $X_{2, t}$ and $X_{3, t}$ in process (4.4) estimated by pDWC. 


$$
\begin{aligned}
p p B_{X_{1}, X_{2}, t}\left(\tau_{j}\right) & =\sum_{B} \widetilde{h}_{j, l} \widetilde{h}_{j, l^{\prime}} a_{1,1}^{(m)}(u) a_{2,2}^{\left(m^{\prime}\right)}(u) \gamma_{X_{1}, X_{2}}\left(t-l-m, t-l^{\prime}-m^{\prime}\right)+ \\
& +\sum_{A} \widetilde{h}_{j, l} \widetilde{h}_{j, l^{\prime}} a_{1,2}^{(m)}(u) a_{2,1}^{\left(m^{\prime}\right)}(u) \gamma_{X_{2}, X_{1}}\left(t-l-m, t-l^{\prime}-m^{\prime}\right) .
\end{aligned}
$$

Notice that $p p F_{X_{1}, X_{2}, t}\left(\tau_{j}\right)$ and $p p B_{X_{1}, X_{2}, t}\left(\tau_{j}\right)$ have two sums, one for region $\mathrm{A}$ and other to region B. Since $\mathbf{X}_{t}$ is a stationary $\operatorname{VAR}(1)$, we can write expressions (4.7) and (4.8) as:

$$
\begin{aligned}
p p F_{X_{1}, X_{2}, t}\left(\tau_{j}\right) & =a_{1,1} a_{2,2} \sum_{l=0}^{L_{j}-1} \sum_{l^{\prime}=0}^{L_{j}-1} \widetilde{h}_{j, l} \widetilde{h}_{j, l^{\prime}} \gamma_{X_{1}, X_{2}}\left(t-l-1, t-l^{\prime}-1\right) \mathbb{I}\left\{l+1>l^{\prime}+1\right\}+ \\
& +a_{1,2} a_{2,1} \sum_{l=0}^{L_{j}-1} \sum_{l^{\prime}=0}^{L_{j}-1} \widetilde{h}_{j, l} \widetilde{h}_{j, l^{\prime}} \gamma_{X_{2}, X_{1}}\left(t-l-1, t-l^{\prime}-1\right) \mathbb{I}\left\{l+1<l^{\prime}+1\right\}= \\
& =a_{1,1} a_{2,2} \sum_{l=1}^{L_{j}-1} \sum_{l^{\prime}=0}^{l-1} \widetilde{h}_{j, l} \widetilde{h}_{j, l^{\prime}} \gamma_{X_{1}, X_{2}}\left(l^{\prime}-l\right)+a_{1,2} a_{2,1} \sum_{l=0}^{L_{j}-2} \sum_{l^{\prime}=l+1}^{L_{j}-1} \widetilde{h}_{j, l} \widetilde{h}_{j, l^{\prime}} \gamma_{X_{2}, X_{1}}\left(l^{\prime}-l\right)= \\
& =a_{1,1} a_{2,2} \sum_{l=1}^{L_{j}-1} \sum_{l^{\prime}=0}^{l-1} \widetilde{h}_{j, l} \widetilde{h}_{j, l^{\prime}} \gamma_{X_{1}, X_{2}}\left(l^{\prime}-l\right)+a_{1,2} a_{2,1} \sum_{l=0}^{L_{j}-2} \sum_{l^{\prime}=l+1}^{L_{j}-1} \widetilde{h}_{j, l} \widetilde{h}_{j, l^{\prime}} \gamma_{X_{1}, X_{2}}\left(-\left(l^{\prime}-l\right)\right)= \\
& =a_{1,1} a_{2,2} F_{X_{1}, X_{2}, t}\left(\tau_{j}\right)+a_{1,2} a_{2,1} B_{X_{1}, X_{2},-t}\left(\tau_{j}\right), \quad \\
& =a_{1,1} a_{2,2} \sum_{l=0}^{L_{j}-1} \sum_{l=0}^{L_{j}-1} \widetilde{h}_{j, l} \widetilde{h}_{j, l^{\prime}} \gamma_{X_{1}, X_{2}}\left(t-l-1, t-l^{\prime}-1\right) \mathbb{I}\left\{l+1<l^{\prime}+1\right\}+ \\
& +a_{1,2} a_{2,1} \sum_{l=0}^{L_{j}-1} \sum_{l=0}^{L_{j}-1} \widetilde{h}_{j, l} \widetilde{h}_{j, l^{\prime}} \gamma_{X_{2}, X_{1}}\left(t-l-1, t-l^{\prime}-1\right) \mathbb{I}\left\{l+1>l^{\prime}+1\right\}= \\
& =a_{1,1} a_{2,2} \sum_{l=0}^{L_{j}-1} \sum_{l^{\prime}=l+1}^{L_{j}-1} \widetilde{h}_{j, l} \widetilde{l}_{j, l^{\prime}} \gamma_{X_{1}, X_{2}}\left(l^{\prime}-l\right)+a_{1,2} a_{2,1} \sum_{l=0}^{L_{j}-1} \sum_{l^{\prime}=0}^{l+1} \widetilde{h}_{j, l} \widetilde{h}_{j, l^{\prime}} \gamma_{X_{2}, X_{1}}\left(l^{\prime}-l\right)= \\
& =a_{1,1} a_{2,2} \sum_{l=0}^{L_{j}-1} \sum_{l^{\prime}=l+1}^{L_{j}-1} \widetilde{h}_{j, l} \widetilde{h}_{j, l^{\prime}} \gamma_{X_{1}, X_{2}}\left(l^{\prime}-l\right)+a_{1,2} a_{2,1} \sum_{l=0}^{L_{j}-1} \sum_{l^{\prime}=0}^{l+1} \widetilde{h}_{j, l} \widetilde{h}_{j, l^{\prime}} \gamma_{X_{1}, X_{2}}\left(-\left(l^{\prime}-l\right)\right)= \\
& =a_{1,1} a_{2,2} B_{X_{1}, X_{2}, t}\left(\tau_{j}\right)+a_{1,2} a_{2,1} F_{X_{1}, X_{2},-t}\left(\tau_{j}\right) . \quad
\end{aligned}
$$

Now we show that $B_{X_{1}, X_{2},-t}\left(\tau_{j}\right)=F_{X_{1}, X_{2}, t}\left(\tau_{j}\right)$ and $F_{X_{1}, X_{2},-t}\left(\tau_{j}\right)=B_{X_{1}, X_{2}, t}\left(\tau_{j}\right)$. Recall that $B_{X Y, t}\left(\tau_{j}\right)$ was defined in (3.2). In (4.9) we have

$$
B_{X_{1}, X_{2},-t}\left(\tau_{j}\right)=\sum_{l=0}^{L_{j}-2} \sum_{l^{\prime}=l+1}^{L_{j}-1} \widetilde{h}_{j, l} \widetilde{h}_{j, l^{\prime}} \gamma_{X_{1}, X_{2}}\left(-\left(l^{\prime}-l\right)\right) .
$$

This quantity have its indexes $l$ and $l^{\prime}$ varying according the Table 4.1. Alternatively, Table 4.1 can be rewritten as Table 4.2.

Considering now Table 4.2 then we have:

$$
B_{X_{1}, X_{2},-t}\left(\tau_{j}\right)=\sum_{l=0}^{L_{j}-2} \sum_{l^{\prime}=l+1}^{L_{j}-1} \widetilde{h}_{j, l} \widetilde{h}_{j, l^{\prime}} \gamma_{X_{1}, X_{2}}\left(-\left(l^{\prime}-l\right)\right)
$$




\begin{tabular}{cll}
\hline$l$ & $l^{\prime}$ & $l-l^{\prime}$ \\
\hline 0 & $1,2, \ldots, L_{j}-1$ & $-1, \ldots,-L_{j}+1$ \\
1 & $2,3, \ldots, L_{j}-1$ & $-1, \ldots,-L_{j}+2$ \\
2 & $3,4, \ldots, L_{j}-1$ & $-1, \ldots,-L_{j}+3$ \\
$\vdots$ & $\vdots$ & $\vdots$ \\
$L_{j}-3$ & $L_{j}-2, L_{j}-1$ & $-2,-1$ \\
$L_{j}-2$ & $L_{j}-1$ & -1 \\
$L_{j}-1$ & & \\
\hline
\end{tabular}

Table 4.1. Variation of values for $l$ and $l^{\prime}$ for term $B_{X_{1}, X_{2},-t}\left(\tau_{j}\right)$ in equation (4.9).

\begin{tabular}{cll}
\hline$l^{\prime}$ & $l$ & $-\left(l^{\prime}-l\right)$ \\
\hline 0 & & \\
1 & 0 & -1 \\
2 & 0,1 & $-1,-2$ \\
3 & $0,1,2$ & $-1,-2,-3$ \\
$\vdots$ & $\vdots$ & $\vdots$ \\
$L_{j}-2$ & $0,1, \ldots, L_{j}-3$ & $-1,-2, \ldots,-L_{j}+2$ \\
$L_{j}-1$ & $0,1, \ldots, L_{j}-3, L_{j}-2$ & $-1,-2, \ldots,-L_{j}+1$ \\
\hline
\end{tabular}

Table 4.2. Alternative form to write Table 4.1.

$$
=\sum_{l^{\prime}=0}^{L_{j}-2} \sum_{l=0}^{l^{\prime}-1} \widetilde{h}_{j, l^{\prime}} \widetilde{h}_{j, l} \gamma_{X_{1}, X_{2}}\left(l^{\prime}-l\right)=F_{X_{1}, X_{2}, t}\left(\tau_{j}\right) .
$$

The proof that $F_{X_{1}, X_{2},-t}\left(\tau_{j}\right)=B_{X_{1}, X_{2}, t}\left(\tau_{j}\right)$ is analogous. Therefore we have

$$
\begin{aligned}
p p F_{X_{1}, X_{2}, t}\left(\tau_{j}\right) & =a_{1,1} a_{2,2} F_{X_{1}, X_{2}, t}\left(\tau_{j}\right)+a_{1,2} a_{2,1} F_{X_{1}, X_{2}, t}\left(\tau_{j}\right)= \\
& =\left(a_{1,1} a_{2,2}+a_{1,2} a_{2,1}\right) F_{X_{1}, X_{2}, t}\left(\tau_{j}\right),
\end{aligned}
$$

and

$$
\begin{aligned}
p p B_{X_{1}, X_{2}, t}\left(\tau_{j}\right) & =a_{1,1} a_{2,2} B_{X_{1}, X_{2}, t}\left(\tau_{j}\right)+a_{1,2} a_{2,1} B_{X_{1}, X_{2}, t}\left(\tau_{j}\right)= \\
& =\left(a_{1,1} a_{2,2}+a_{1,2} a_{2,1}\right) B_{X_{1}, X_{2}, t}\left(\tau_{j}\right) .
\end{aligned}
$$

In other words, for a bivariate stationary $\operatorname{VAR}(1)$, the pDWC components are proportional to the DWC from Chapter 3, weighted by a function of the VAR coefficients.

We now present three other examples of VAR(1) processes simulated considering the model parameterization in (4.3). In all three examples we simulated the proposed models with 100 replications and $T=1024$ as the length of the simulated time series to calculate the average $p p F_{X_{1}, X_{2}, t}\left(\tau_{j}\right)$ and the $p p B_{X_{1}, X_{2}, t}\left(\tau_{j}\right)$ and their standard errors.

Example 4.3. In this example we consider the following model:

$$
\mathbf{X}_{t}=\left(\begin{array}{cc}
0.0 & 0.5 \\
0.5 & 0.0
\end{array}\right) \mathbf{X}_{t-1}+\boldsymbol{\epsilon}(t)
$$


So we expect $p p F$ and $p p B$ to be very close, since the influences between $X_{1, t}$ and $X_{2, t}$ on each other are the same. Figure 4.5 present the average $p p F$ and $p p B$ for 100 generated time series and, as expected, the curves for the average $p p F$ and $p p B$ are very similar. Notice the term in $a_{11} a_{22}$ in (4.11) and (4.12) disappears since $a_{11}=a_{22}=0$.
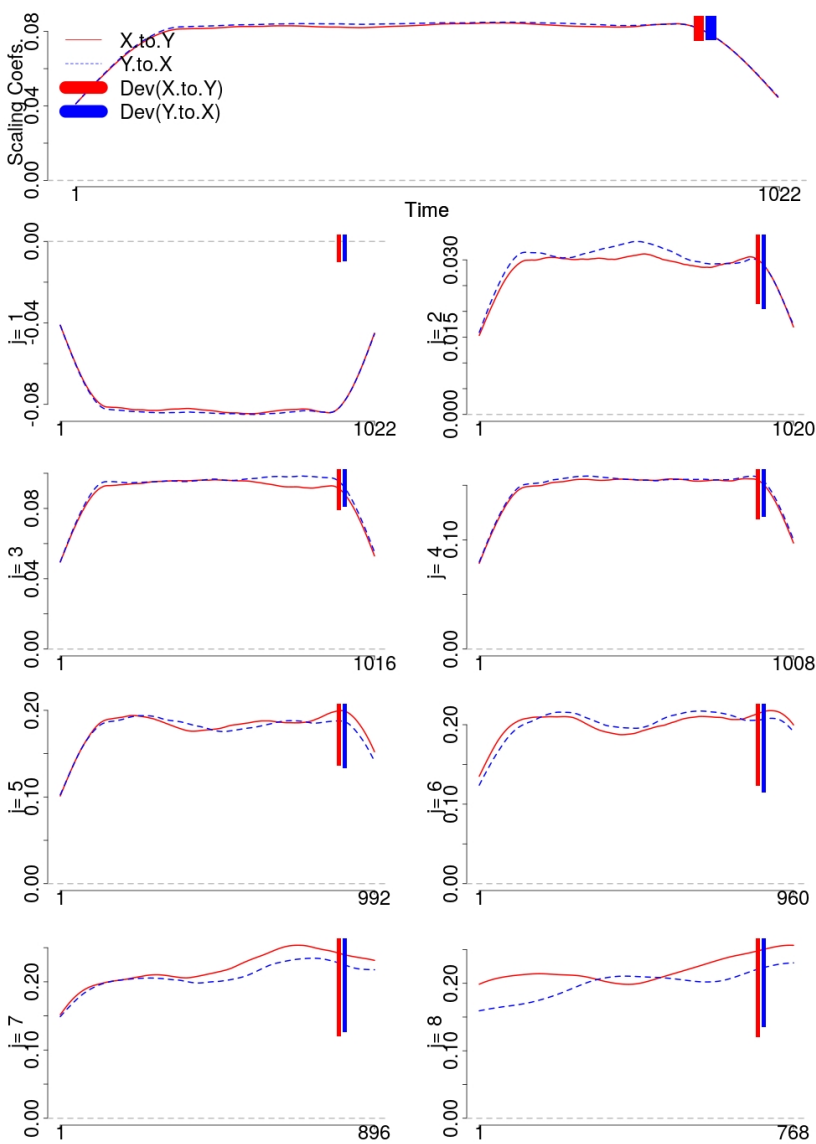

Figure 4.5. Average Partial Directed Wavelet Covariance (ppDWC) for Example 4.3.

Example 4.4. In this example we consider the following model:

$$
\mathbf{X}_{t}=\left(\begin{array}{cc}
0.4 & -0.3 \\
0.3 & 0.3
\end{array}\right) \mathbf{X}_{t-1}+\boldsymbol{\epsilon}(t)
$$

i.e. we now simulate the relations between $X_{1, t}$ and $X_{2, t}$ with inverted signs and also with autodependency in both time series. Figure 4.6 shows the results for the average $p p F$ and $p p B$ with 100 random generated time series. As we can see, the average quantities $p p F$ and $p p B$ for this simulation can capture the inverted signs for the influences $X_{1} \rightarrow X_{2}$ and $X_{2} \rightarrow X_{1}$.

Example 4.5. In this simulation we cause a stronger influence from $X_{2, t-1}$ to $X_{1, t}$ than from $X_{1, t-1}$ to $X_{2, t}$.

$$
\mathbf{X}_{t}=\left(\begin{array}{cc}
0.0 & 0.8 \\
0.3 & 0.0
\end{array}\right) \mathbf{X}_{t-1}+\boldsymbol{\epsilon}(t) .
$$




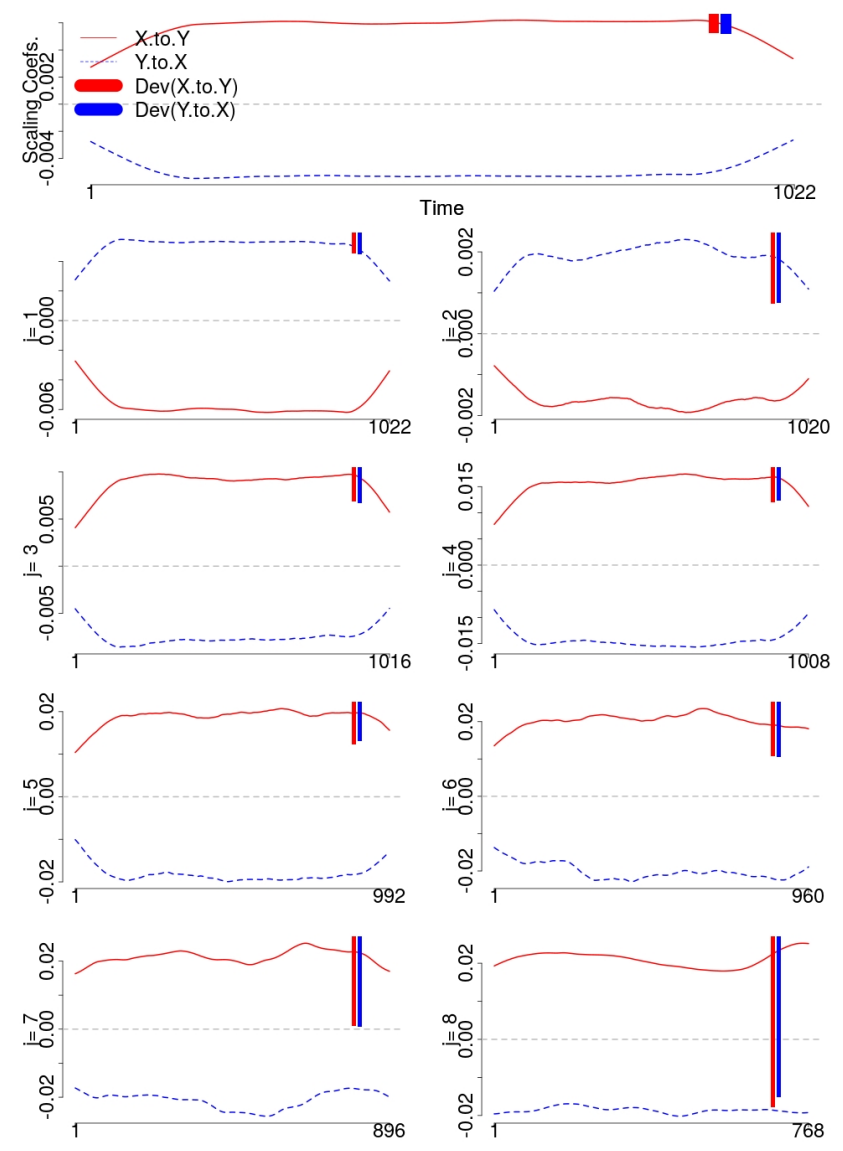

Figure 4.6. Average Partial Directed Wavelet Covariance (ppDWC) for Example 4.4.

Figure 4.7 presents the graphs for the average $p p F$ and $p p B$ using 100 replications. The quantities can capture the absolute difference between the two simulated relations: the average $p p F$ are above average of $p p B$ (in absolute values) in all scales.

Example 4.6 (ppDWC for a trivariate VAR(1)). Recall the following model from Example 4.1:

$$
\mathbf{X}_{t}=\mathbf{A X}_{t-1}+\boldsymbol{\epsilon}(t)=\left(\begin{array}{ccc}
0.0 & 0 & 0.6 \\
0.5 & 0 & 0.3 \\
0.0 & 0.3 & 0.3
\end{array}\right) \mathbf{X}_{t-1}+\boldsymbol{\epsilon}(t), t \in 1, \ldots, 1024
$$

with $\boldsymbol{\epsilon}(t)$ as in (3.7). The relationship between the components is illustrated in Figure 4.1.

Figures 4.8 to 4.10 present the average ppDWC estimated by 100 replications of the process 4.4 using $\mathrm{T}=1024$. In this example, we considered the coefficients of the tv-VAR as unknown. Therefore we estimated then by using a moving window $(M=256)$ along the time series, as we did in Chapter 2 for the PDC with moving windows. The graphs cannot illustrate well the relations between $X_{1}$ and $X_{2}$ as the pDWC using the linear filter illustrated in Figures 4.2 to 4.4. On the other hand, the scaling filters for relations of $X_{2}$ and $X_{3}$ present results close to the expected. This suggests the ppDWC is sensible to the estimation of the tv-VAR coefficients. 


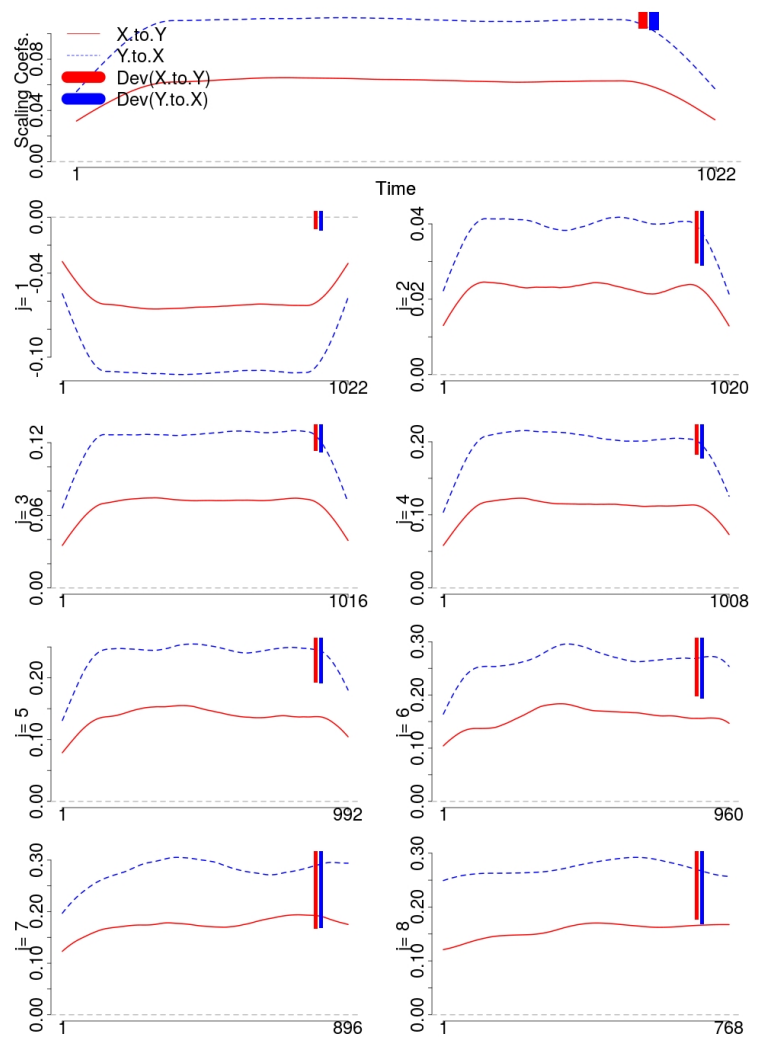

Figure 4.7. Average Partial Directed Wavelet Covariance (ppDWC) for Example 4.5.
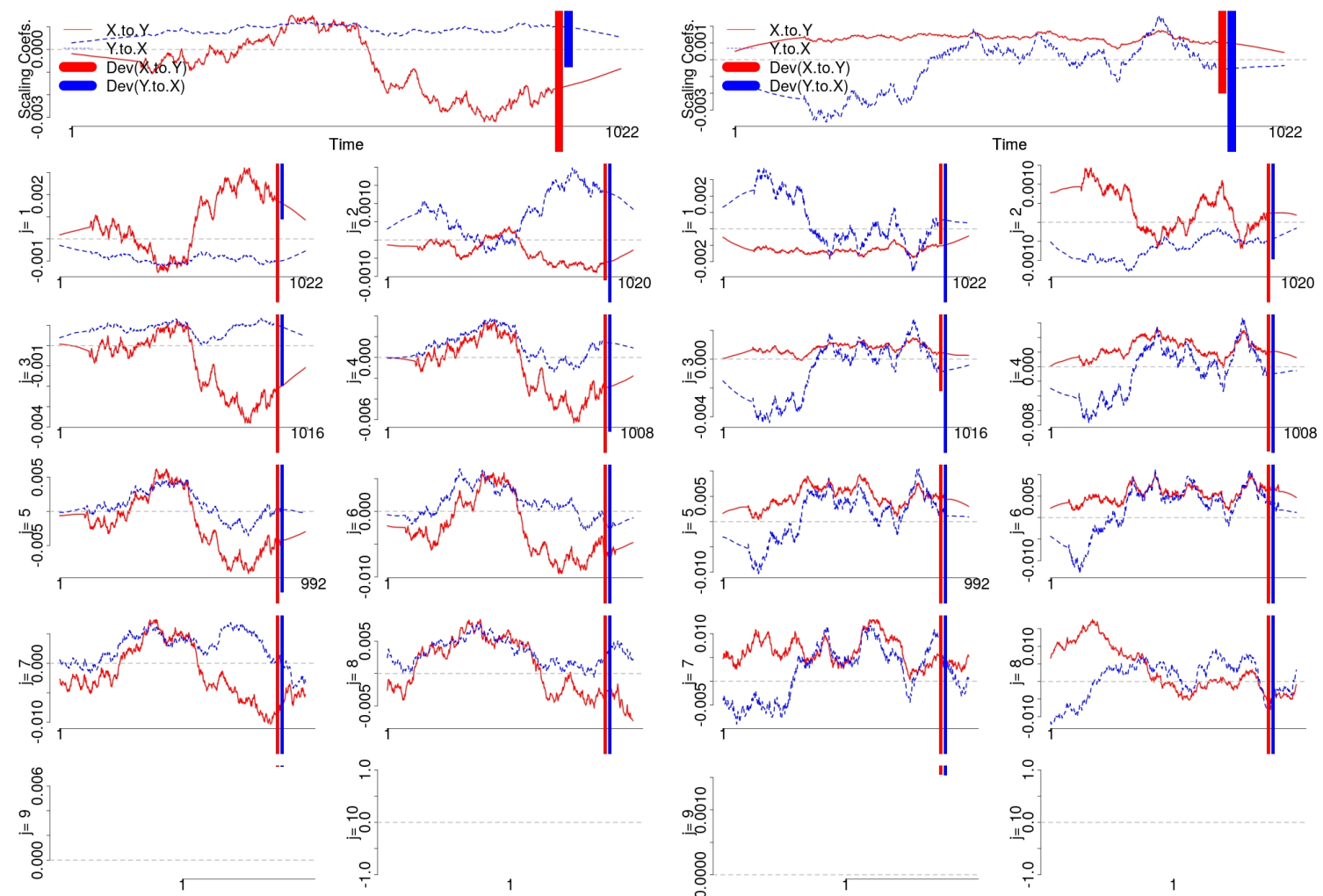

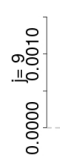

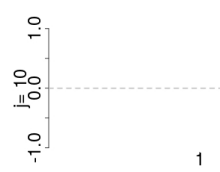

Figure 4.8. Example 4.6: Relations between com- Figure 4.9. Example 4.6: Relations between components $X_{1, t}$ and $X_{2, t}$ in process (4.4) estimated by ponents $X_{1, t}$ and $X_{3, t}$ in process (4.4) estimated by $p p D W C$. $p p D W C$. 


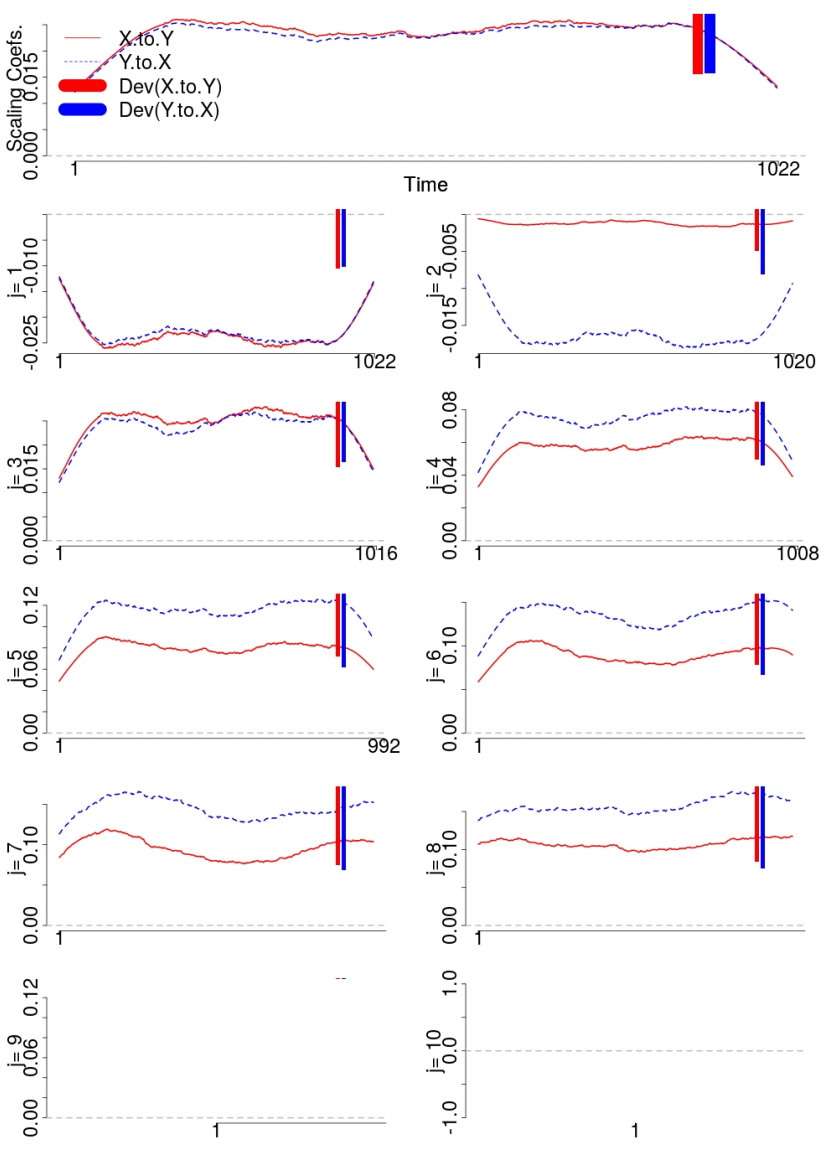

Figure 4.10. Example 4.6: Relations between components $X_{2, t}$ and $X_{3, t}$ in process (4.4) estimated by ppDWC.

In this chapter we proposed methodologies to measure the relation between two components of a vector $\mathbf{X}_{t, T}, X_{t, T}^{(1)}$ and $X_{t, T}^{(2)}$ after removing the influence of other components. Notice that Proposition 4.1 assumes that the process is a tv-VAR(p), and estimating the model can be complicated in certain situations. Sato et al. (2006) proposed a tv-VAR(p) approach based on wavelets. Starting from the wavelet representation of the tv-VAR(p), the authors estimated its coefficients and proved asymptotic normality of the estimators. Another approach is consider a sequence of VAR(p) estimated in a moving window of size $m<T$ as presented in Lopes (2014) for the PDC. This technique was used in Example 4.6 to estimate the tv-VAR(1).

The two quantities proposed in this chapter, pDWC and ppDWC, are alternatives to the DWC when we have a locally stationary multivariate process with multiple influences between the components. While the pDWC is calculated using a linear filter, its assumptions over the process are more flexible than the ppDWC; this later assumes that the process is a tv-VAR(p) and estimating the time-varying coefficients can be complex and can impact the ppDWC efficiency. The estimators for $p p F($.$) and$ $p p B($.$) were calculated replacing a_{i, j}^{m}(u), i, j \in\{1, \ldots N\}, m=\{1, \ldots, p\}, u \in[0,1]$, in (B.10) and (B.11) by their MLE estimators and $\gamma_{X_{1}, X 2}$ by (3.3).

We proposed the ppDWC based on the assumption that the process is represented by a tv-VAR model. Although this assumption is very reasonable, the expectation of the ppDWC estimator becomes a quantity very difficult to calculate since it depends on the tv-VAR coefficient estimators. 


\section{Chapter 5}

\section{Simulations}

In this chapter we first present two simulations for bivariate processes with different local covariance structures, each one representing changes in $X_{t, T}$ and $Y_{t, T}$ relationships. The main difference between them is how fast these changes on covariance structure happen: the first simulation shows a drastic shift - segmented covariance structures - while the second has a smooth variation - according to a cosine function. The main goal in these two simulations is to compare PDC, WTC and DWC. Simulation 3 considers a trivariate process with different influences within the components of the process. In this simulation we compare all presented methodologies i.e., PDC,WTC, DWC, pDWC and ppDWC. The main goal is to evaluate the efficiency of each methodology on multivariate analysis of locally stationary processes. We used Haar wavelets in all analyses of this chapter.

\subsection{Simulation 1}

Consider a bivariate model given by:

$$
\begin{gathered}
\left(\begin{array}{c}
X_{t, T} \\
Y_{t, T}
\end{array}\right)=\sum_{i=1}^{3} \phi_{i}\left(\begin{array}{c}
X_{i, t-1, T} \\
Y_{i, t-1, T}
\end{array}\right) \mathbb{I}\left(T_{i}\right)+\left(\begin{array}{c}
\epsilon_{1, t} \\
\epsilon_{2, t}
\end{array}\right), \\
\phi_{1}=\left(\begin{array}{ll}
0.5 & 0.0 \\
0.0 & 0.3
\end{array}\right), \phi_{2}=\left(\begin{array}{cc}
0.5 & 0.5 \\
0.0 & 0.3
\end{array}\right), \phi_{3}=\left(\begin{array}{cc}
0.5 & 0.0 \\
0.5 & 0.3
\end{array}\right),
\end{gathered}
$$

$\epsilon_{1, t}, \epsilon_{2, t}$ are independent white noises with $\sigma_{\epsilon_{1}}=\sigma_{\epsilon_{2}}=1$ and $T_{1}=\left\{t: t \in\left[\begin{array}{lll}1 & : 342\end{array}\right\}\right.$, $T_{2}=\{t: t \in[343: 685]\}$ and $T_{3}=\{t: t \in[686: 1028]\}, T=1028$.

In this simulation $X_{t, T}$ and $Y_{t, T}$ are uncorrelated until time $t=343$. From this time to $t=685, X_{t, T}$ depends on past $Y_{t-1, T}$ and for $686 \leq t \leq 1028$ is $Y_{t, T}$ that depends on $X_{t-1, T}$. Figure 5.1 presents one of the 100 simulated time series based on this model.

Next we calculate the Directed Coherence (DC), the Wavelet Coherence (WTC) and the Directed Wavelet Covariance (DWC) for the 100 simulated time series. We chose one of the 100 time series to present graphs for each one of these quantities. We also calculate the average quantities - Directed Coherence, Wavelet Coherence and Directed Wavelet Covariance - for the 100 simulated time series. As in Section 2.2, the Directed Coherence is a particular case of PDC when we have a bivariate process.

Figure 5.2 presents Wavelet Coherence (WTC) and significant relations between the two time series. The average phase for significant regions considering only positive phases is $52.25 \pm 36.22(35.34)$ 


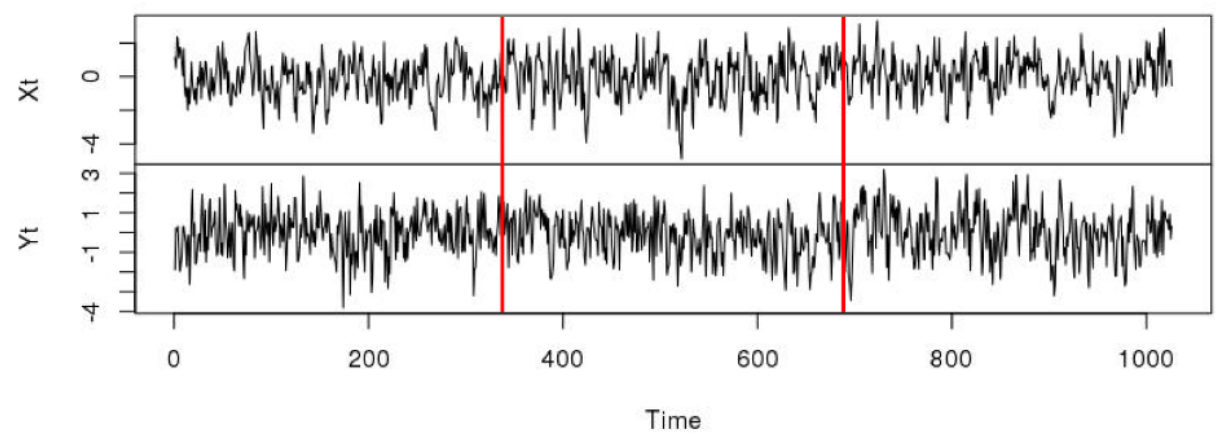

Figure 5.1. One of the simulated Time Series in Simulation 1: the vertical red bars are the times $t=343$ and $t=686$, when the shifts in covariance structure occur. Notice that it's not possible to see the shift in the dependence by only looking at the time series.

and $-68.48 \pm 44.22(44.2)$ if we consider only negative phases. The value after " \pm " represents the circular standard deviation (Grinsted et al. (2004)) and the value within the parenthesis is the linear standard deviation.

Graphs (a) and (b) in Figure 5.3 shows the Directed Coherence with an overlapped moving win-
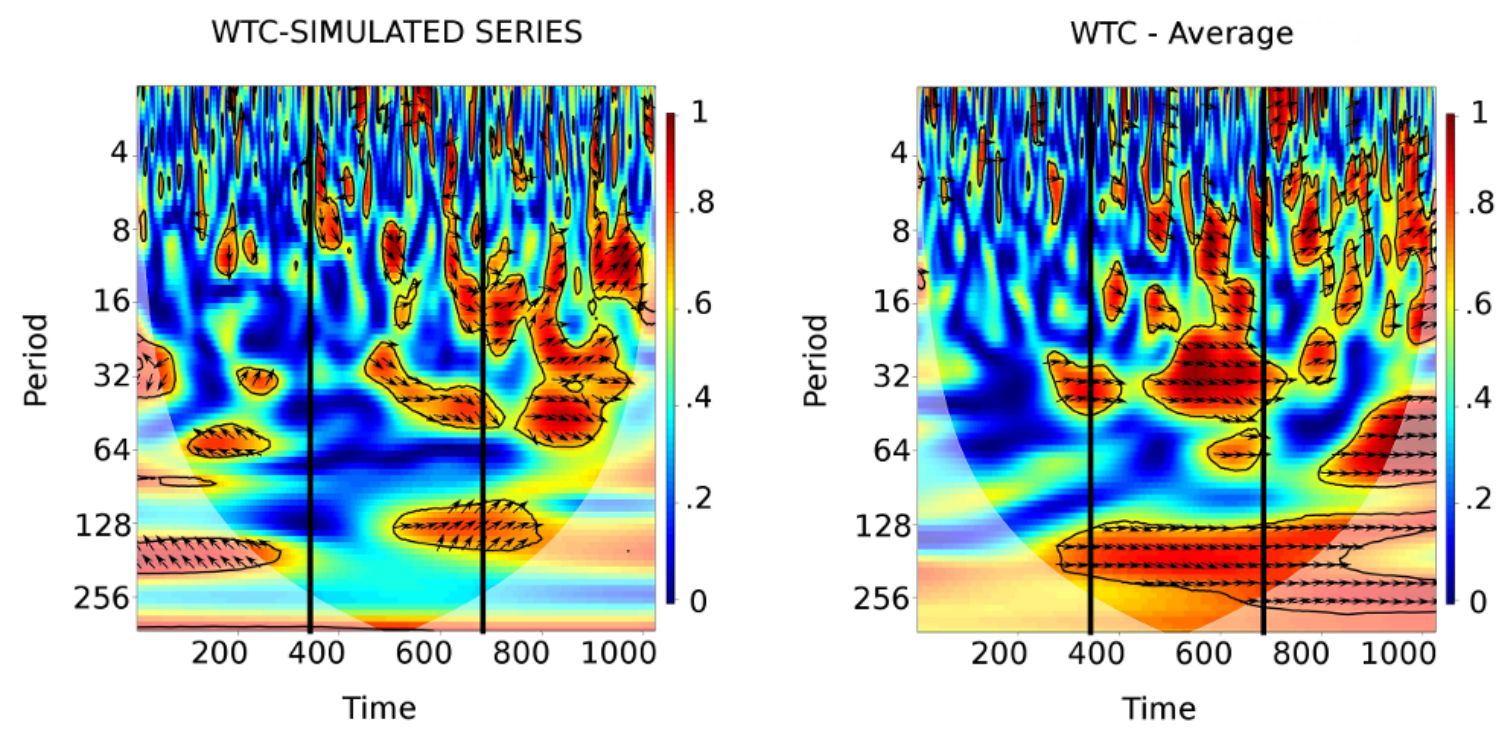

Figure 5.2. Simulation 1: WTC for one of the simulated time series (graph on the left - randomly selected) and average WTC for the 100 simulated time series (graph on the right). Arrows pointing down in central time and scale 32 suggest influences from $Y_{t}$ to $X_{t}$ while arrows pointing up in the last period for smaller scales suggest influences from $X_{t}$ to $Y_{t}$. The average WTC suggests relation $X \leftarrow Y$ also for smoothest (long-term) scales. The vertical black bars are the times $t=343$ and $t=686$, when the shifts in covariance structure occur.

dow with $\mathrm{M}=128$ for a randomly chosen replication (118 overlapped observations, i.e., each window jumps ten observation ahead). The DC with moving windows is an alternative to non-stationary processes and also can identify influences $X \rightarrow Y$ from window 63 ( $\mathrm{t}=630$ ) until the end of the period 

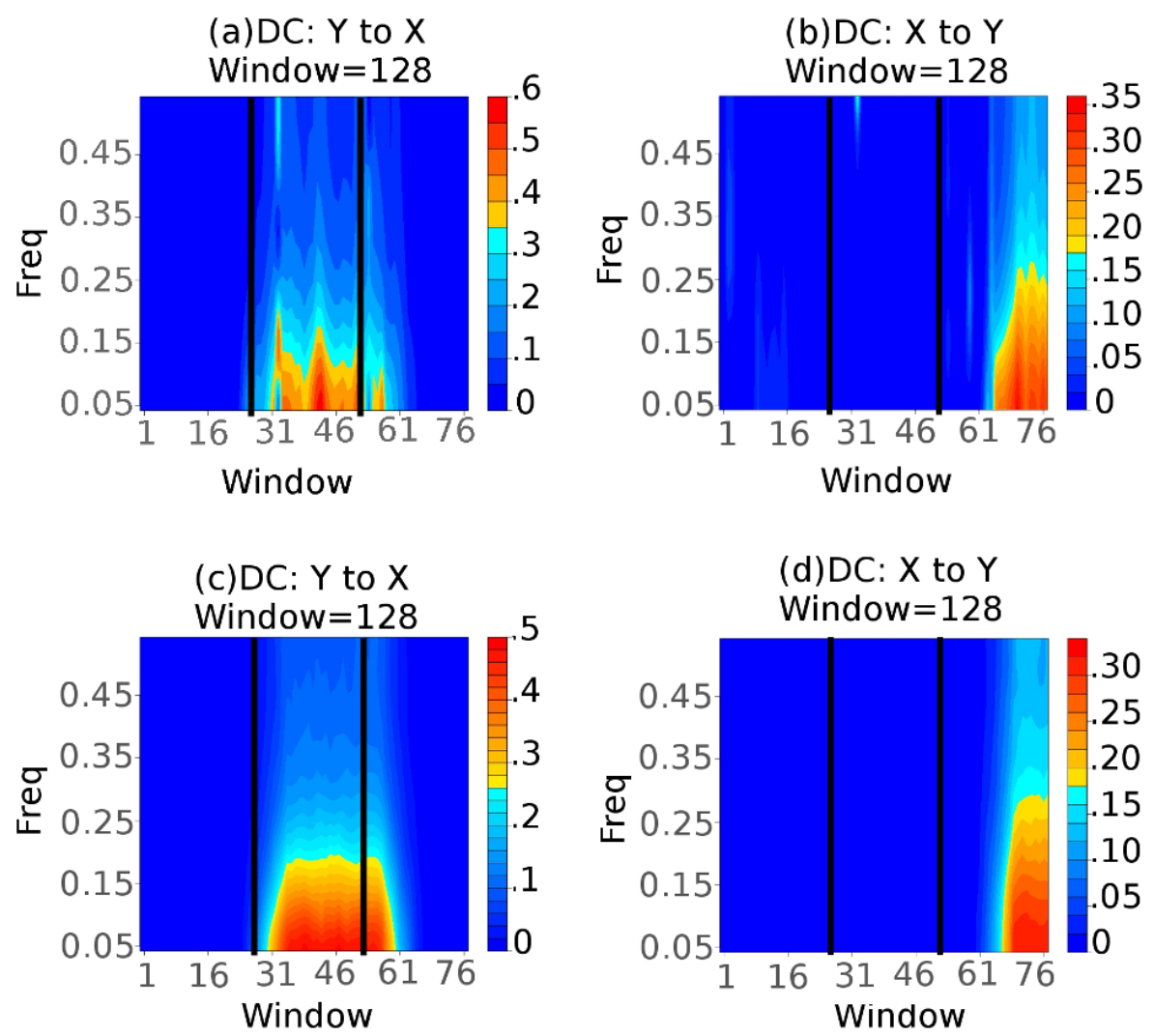

Figure 5.3. DC for Simulation 1: The Directed Coherence is estimated using moving windows of size $M=128$ and the estimated quantities can capture the simulated dependency between $X_{t, T}$ and $Y_{t, T}$. (a) and (b) are the Directed Coherence for one particular simulated time series and $(c)$ and $(d)$ are the average Directed Coherence for 100 simulations. The vertical black bars are the times $t=343$ and $t=686$, when the shifts in covariance structure occur.

and $Y \rightarrow X$ starting from window 31 (time=310) until time 600. The relations $X \rightarrow Y$ and $Y \rightarrow X$ were stronger in low frequencies and decay in higher frequencies. This is expected since the simulated processes are VAR(1). Graphs (c) and (d) shows the average Directed Coherence for the 100 simulated time series and we can see the conclusions are the same.

Notice that Directed Coherence assumes a stationary VAR(p) with $p$ chosen by AIC criteria for each interval of width $M=128$. To avoid instability on the algorithm, we restricted the maximum AR order to $\mathrm{p}=10$. Otherwise, it over-parameterizes the fitted models in some windows.

Figure 5.4 presents the Directed Wavelet Covariance (DWC) calculated for a randomly chosen simulated time series and Figure 5.5 shows the average DWC by scale for 100 replications. We estimated the DWC using Haar wavelets, Epanechnikov kernel and $b_{T}=0.3$. The dependency relation between $X_{t}$ and $Y_{t}$ is evident in almost all scales. As expected, central coefficients present strong relation from $Y_{t}$ to $X_{t}$ and the last coefficients in each scale can capture the relation from $X_{t}$ to $Y_{t}$. Notice that each scale has $2 L_{j}$ coefficients excluded due to circular convolution.

Now given one of the simulations, we want to test if the DWC process is similar to the DWC of a stationary $\operatorname{VAR}(1)$ as in (2.16) with $p=1, a_{12}(1)=0$ and $a_{21}(1)=0$. We estimate the $\operatorname{VAR}(1)$ parameters and evaluate the significance of the observed DWC by bootstrap. The bootstrap helps us to identify the causal relation between the components. By setting upper and lower boundaries 

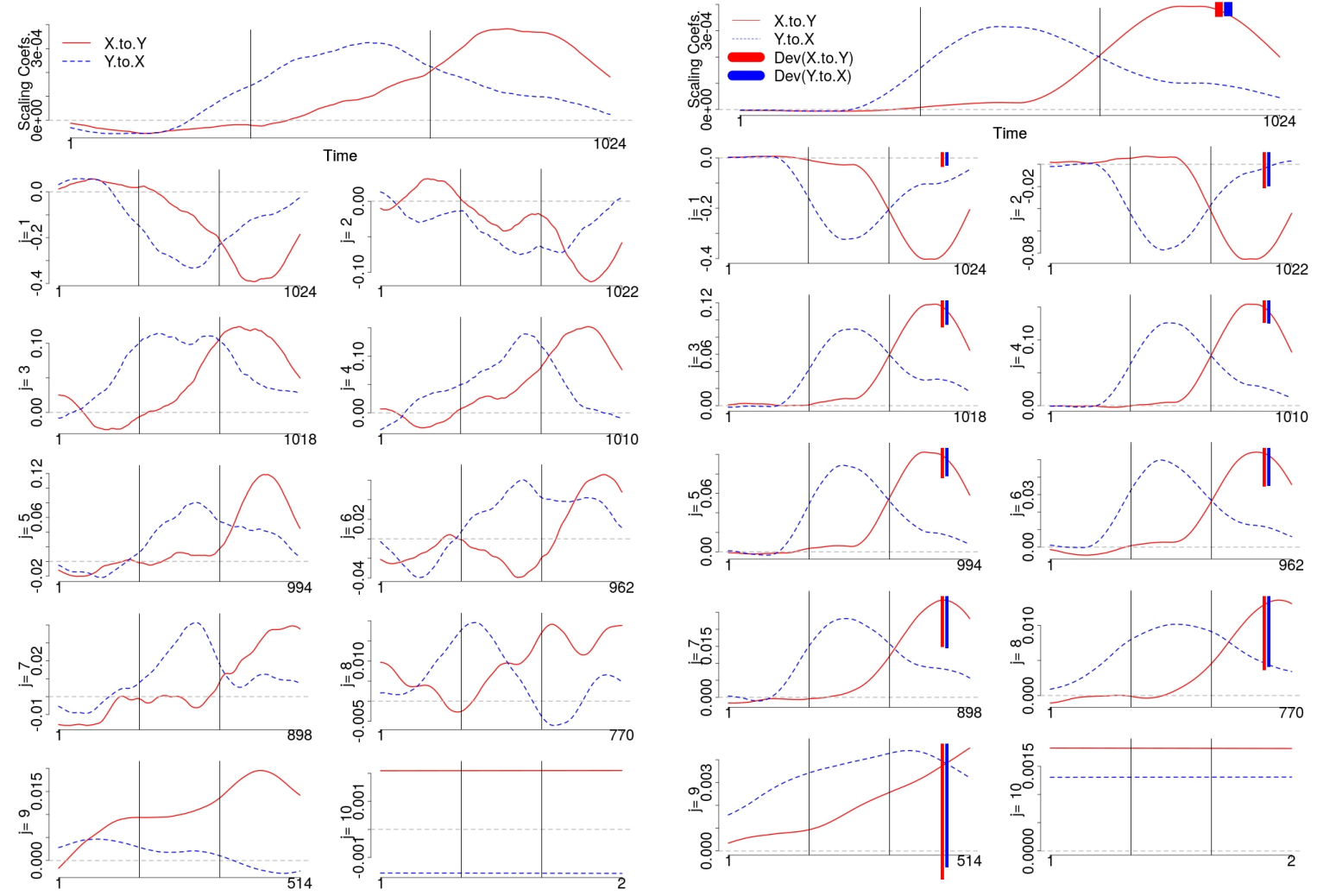

Figure 5.4. Directed Wavelet Covariance for one time series of Simulation 1 estimated using Haar wavelets, Epanechnikov kernel and $b_{T}=0.3$. Scaling coefficients and central scales capture better the relation between the simulated components. The vertical black bars are the times $t=343$ and $t=686$, when the shifts in covariance structure occur.
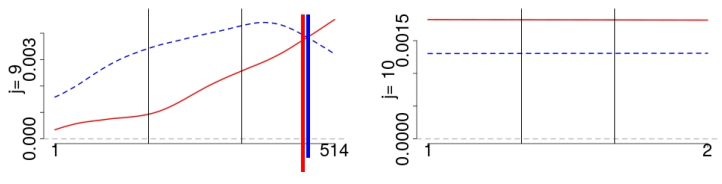

Figure 5.5. Average Directed Wavelet Covariance for 100 time series in Simulation 1. Central coeffcients in all scales suggest high influence from $Y_{t, T}$ to $X_{t, T}$. This relation is inverted in the last third of the observed period. The red and blue bars represent the average standard deviations for $\widehat{F}_{X Y, t}\left(\tau_{j}\right)$ and $\widehat{B}_{X Y, t}\left(\tau_{j}\right)$ respectively, i.e. $\sum_{t} S D\left(\widehat{F}_{X Y, t}\left(\tau_{j}\right)\right) /(T-$ $\left.L_{j}\right)$ and $\sum_{t} S D\left(\widehat{B}_{X Y, t}\left(\tau_{j}\right)\right) /\left(T-L_{j}\right)$.

to the Directed Wavelet Covariance using empirical distributions, we can identify where the causal pattern between the simulated components is significant. We used 100 bootstrap samples and Figure 5.6 shows $\widehat{F}_{X Y, t}\left(\tau_{j}\right)$ and $\widehat{B}_{X Y, t}\left(\tau_{j}\right)$ for the simulated time series with the significant regions (dashed line inner regions) considering the empirical $2.5 \%$ and $97.5 \%$ quantiles. The conclusions corroborate the result presented in Figures 5.4 and 5.5.

\subsection{Simulation 2}

In this second simulation we also have a time-varying covariance structure but now according to a cosine function (Figure 5.7.a). The simulated model is the following:

$$
\begin{aligned}
X_{t, T} & =X_{t-1, T}+\epsilon_{1, t} \\
Y_{t, T} & =2 \cdot \cos \left(\frac{2 \pi t}{T}\right) X_{t-1, T}+\epsilon_{2, t}
\end{aligned}
$$



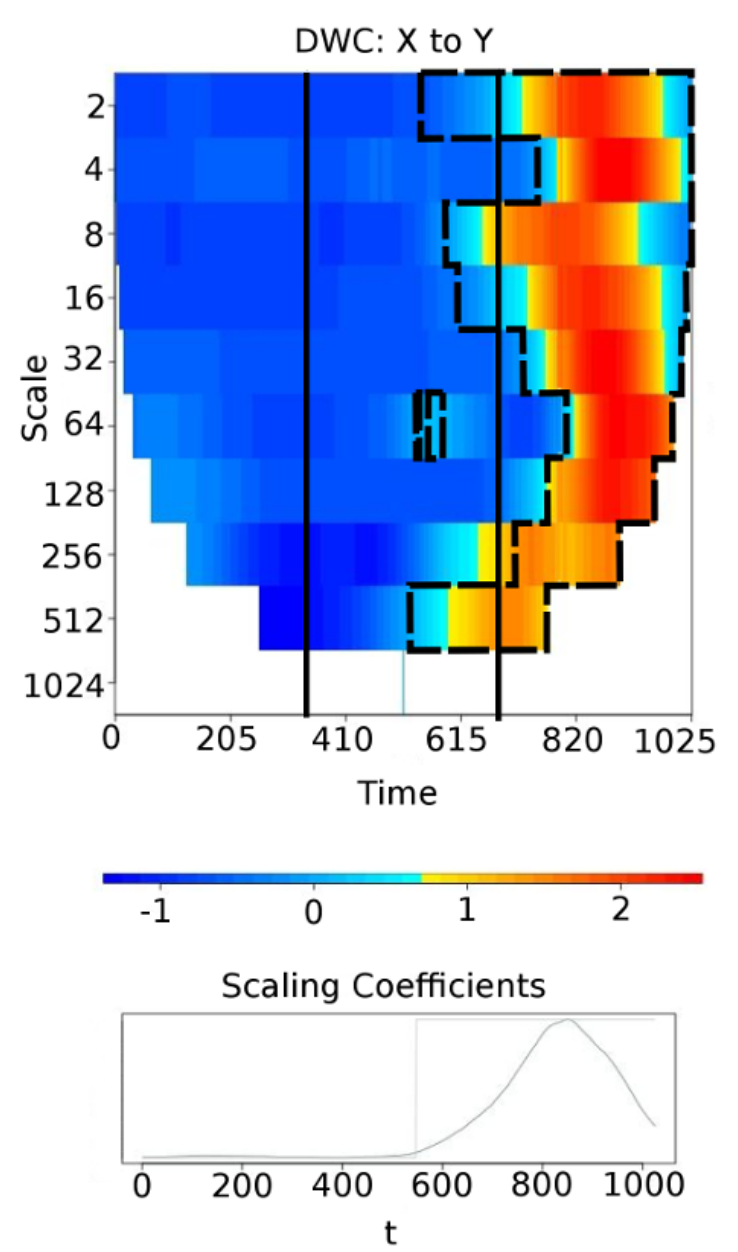
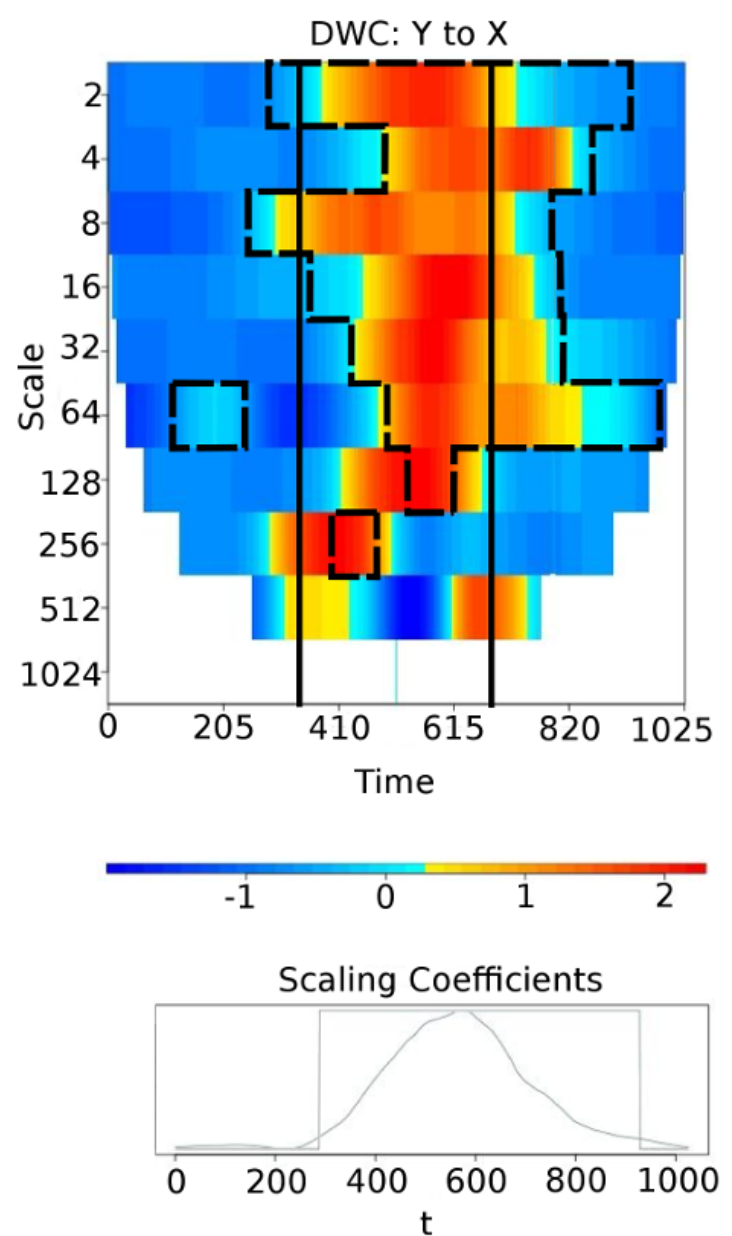

Figure 5.6. Bootstrap for $D W C$ in for Simulation 1. Regions are significant with confidence level $95 \%$ considering the empirical distribution of $D W C$ by bootstrap procedure, supposing the simulated time series is a stationary $\operatorname{VAR}(1)$. Notice that the significant regions are equivalent to the regions with the simulated dependency between $X_{t, T}$ and $Y_{t, T}$. The vertical black bars are the times $t=343$ and $t=686$, when the shifts in covariance structure occur.

where $\epsilon_{1, t} \sim \operatorname{iidN}(0,1), \epsilon_{2, t} \sim \operatorname{iidN}\left(0,0.5^{2}\right), \operatorname{cov}\left(\epsilon_{1, t}, \epsilon_{2, t^{\prime}}\right)=0, \forall t, t^{\prime}=1, \ldots, 1024 . \mathrm{T}=1024$.

Figures 5.8 to 5.10 show that all three methodologies can capture the relations between $X_{t, T}$ and $Y_{t, T}$, with a strong dependency from $X_{t, T}$ to $Y_{t, T}$ and almost none from $Y_{t, T}$ to $X_{t, T}$. Notice that all the estimations are more precise and accurate in this simulation rather than in Simulation 1. The main reason for that is the presence of a more subtle change of dependency pattern according to the cosine function versus the drastic changes presented in Simulation 1. 
(a)

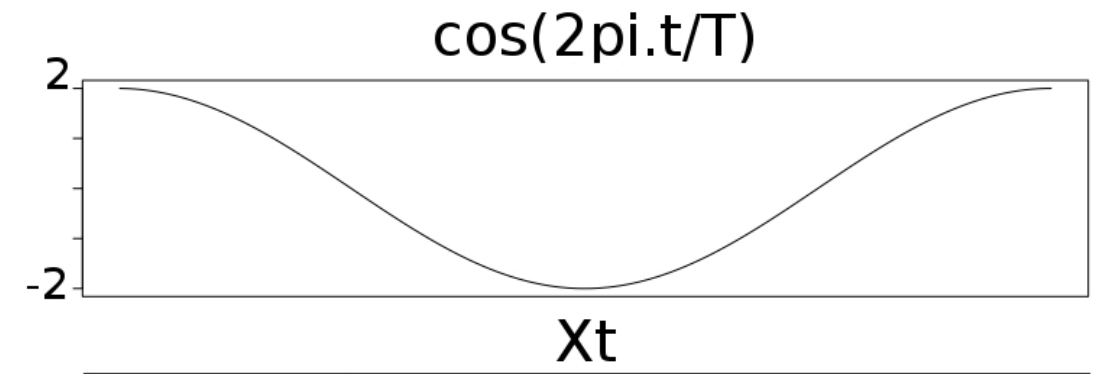

(b)

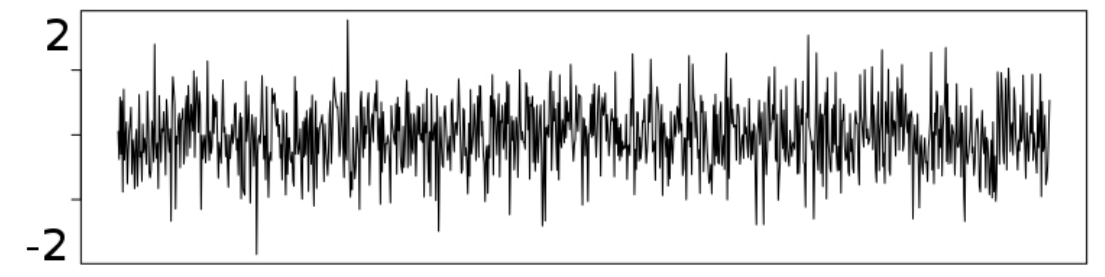

(c)

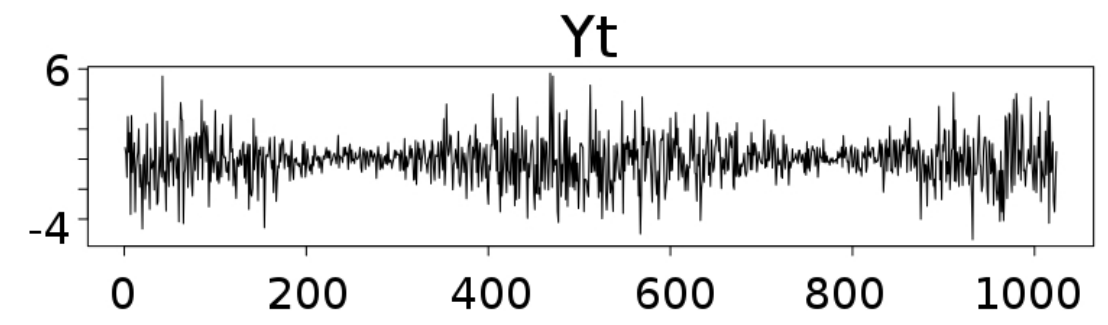

Time

Figure 5.7. Simulation 2: (a) $2 \cos (2 \pi t / T)$ and simulated time series ( $b$ and $c$ ). In this simulation, only $Y_{t, T}$ is dependent on $X_{t, T}$ according to the magnitude of a cosine function.
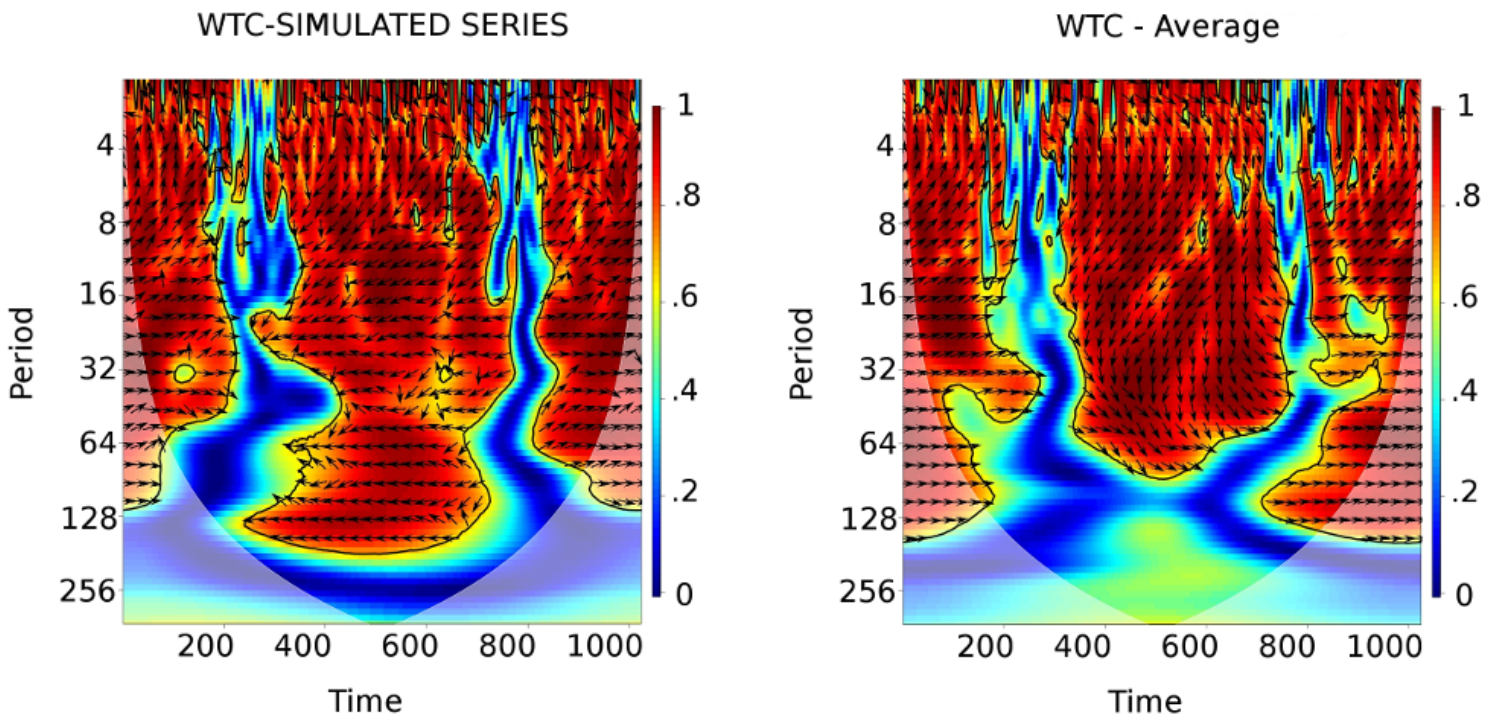

Figure 5.8. WTC for Simulation 2: Graph on the left is Wavelet Coherence for one of the simulated time series and the right graph is the average Wavelet Coherence for the 100 simulated time series. The relation between the series is not significant only in regions that cos(.) is close to zero. 

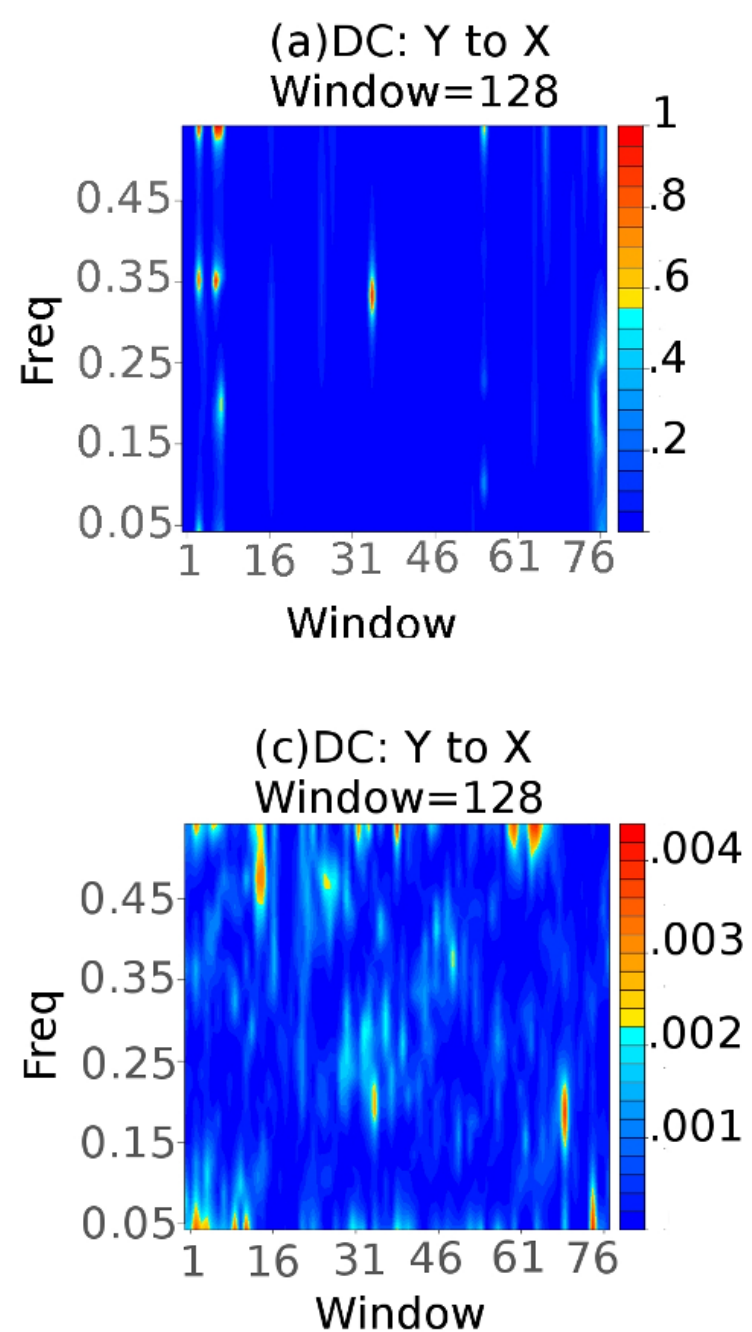

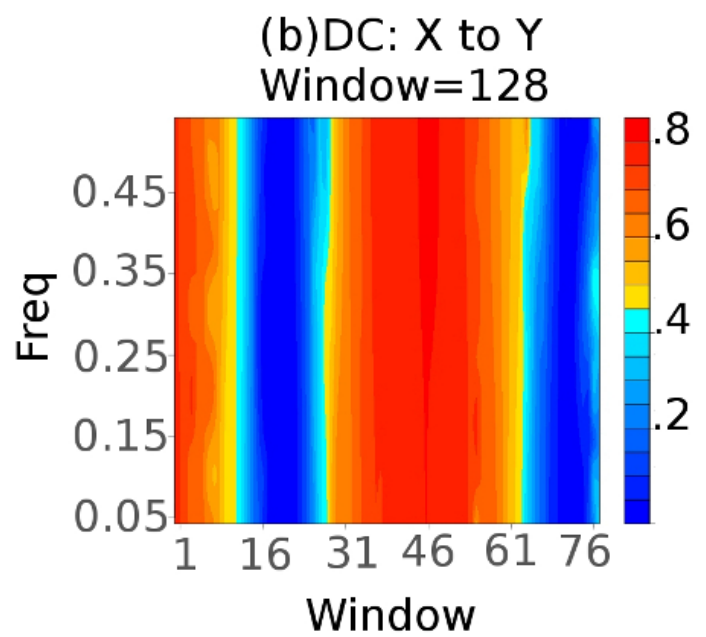

(d)DC: $X$ to $Y$

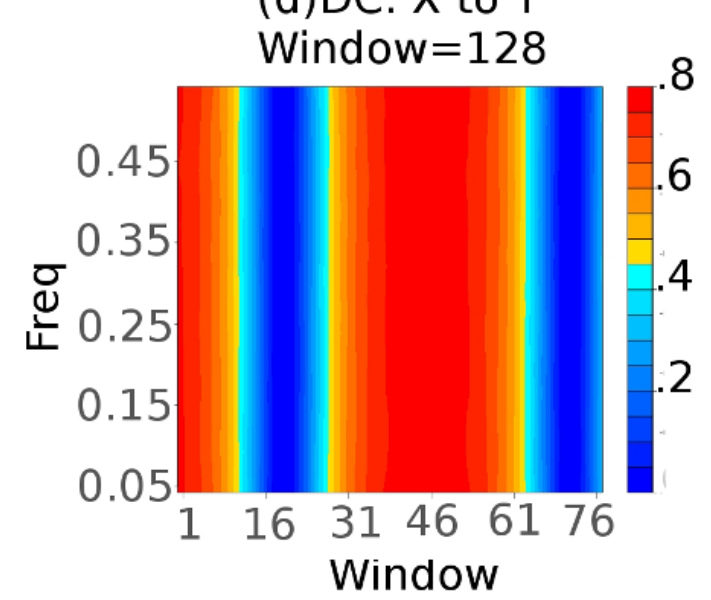

Figure 5.9. $D C$ for Simulation 2: The Directed Coherence is estimated using moving windows of size $M=128$ and the estimated quantities can capture the simulated dependency between $X_{t, T}$ and $Y_{t, T}$. (a) and (b) are the Directed Coherence for a randomly chosen simulated time series and $(c)$ and $(d)$ are the average Directed Coherence for 100 replications. We can see the strong influence from $X_{t, T}$ to $Y_{t, T}$ except in the regions where $\cos ($.$) is close to zero. As expected, there is almost no influence from Y_{t, T}$ to $X_{t, T}$. 

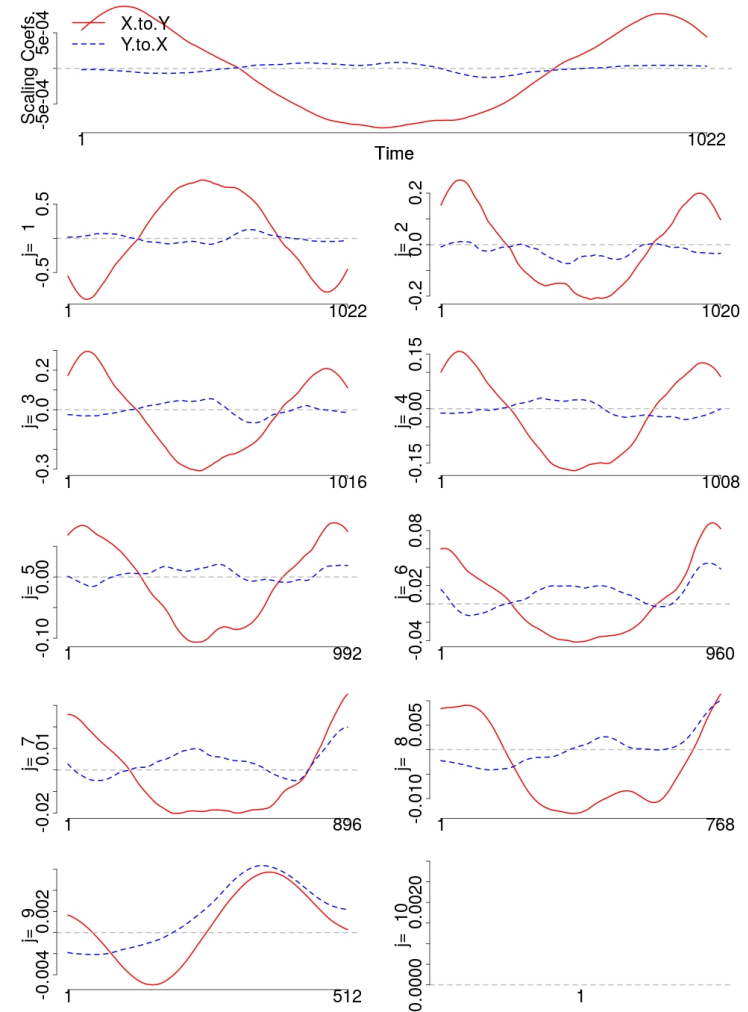

Figure 5.10. Directed Wavelet Covariance for 1 time series of Simulation 2 estimated using Haar wavelets, Epanechnikov kernel and $b_{T}=0.3$.
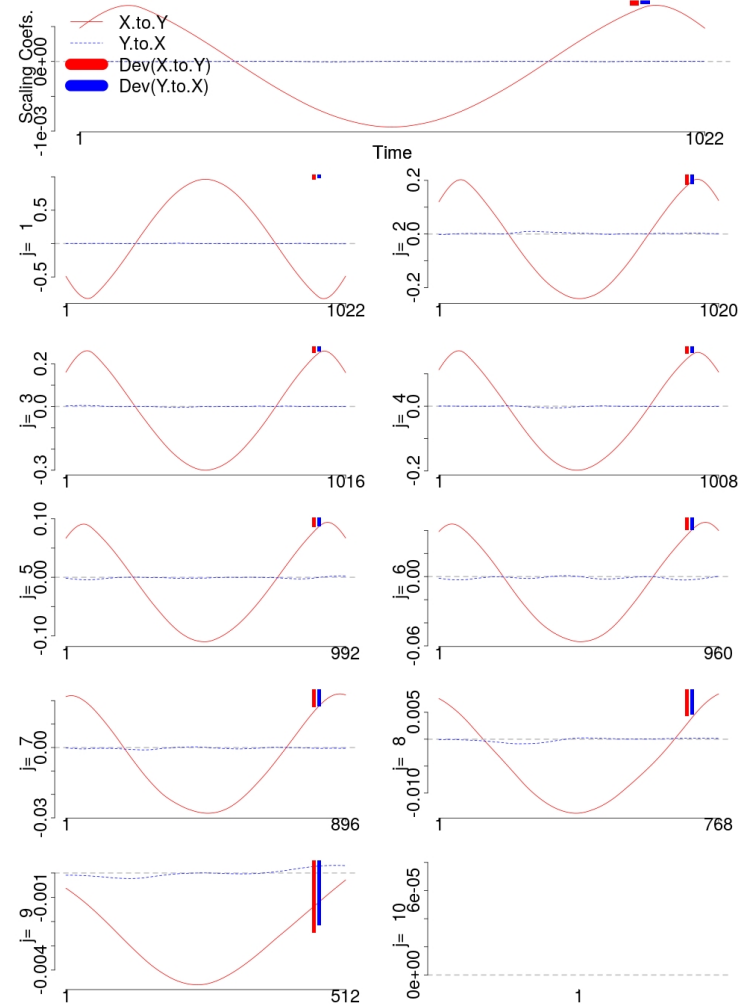

Figure 5.11. Average Directed Wavelet Covariance for 100 replications. We see the cosine simulated relation from $X_{t, T}$ to $Y_{t, T}$ is detected by the Directed Wavelet Covariance in all scales. 


\subsection{Simulation 3}

In this simulation we consider 100 simulated time series of a time-varying VAR(1) process. We used $\mathrm{T}=1024$ to simulate

$$
\mathbf{X}_{t, T}=\mathbf{A}(t, T) \mathbf{X}_{t-1, T}+\boldsymbol{\epsilon}(t)
$$

$\boldsymbol{\epsilon}(t)=\left(\epsilon_{1, t}, \epsilon_{2, t}, \epsilon_{3, t}\right)^{\top} \sim W N(\underset{\sim}{0}, \boldsymbol{\Sigma})$ is a bivariate white noise with independent components and covariance matrix $\boldsymbol{\Sigma}=I_{3} . \mathbf{A}(t, \widetilde{T})$ is a $3 \times 3$ time-varying matrix given by:

$$
\mathbf{A}(t, T)=\left(\begin{array}{ccc}
0.5 & 0 & 0.5 \cos (6 \pi t / T) \\
0.5 \cos (2 \pi t / T) & 0 & -0.6 \mathbb{I}(t \leq 512)+0.3 \mathbb{I}(t>512) \\
0.5 \cos (20 \pi t / T) & 0.5 \mathbb{I}(t \leq 512)-0.5 \mathbb{I}(t>512) & 0
\end{array}\right) .
$$

Figure 5.12 shows graphs of the coefficients functions over time and Figure 5.13 presents an example of one simulated time series of process (5.3). Notice that it is not possible to identify the inherited dependency between the components only by looking to the time series. We will then calculate the studied measures: PDC, WTC and the proposed: DWC, pDWC and ppDWC to the 100 replications and calculate the average curve for each one of these quantities. We used $b=0.3$ as bandwidth for covariance estimation in (3.3) and Epanechnikov Kernel. For both moving PDC and tv-VAR estimation on ppDWC we used $\mathrm{M}=256$ as window size. We considered $p=10$ as maximum lag on PDC estimation and AIC criterion to choose the order of the model. For ppDWC we considered $\mathrm{p}=1$.
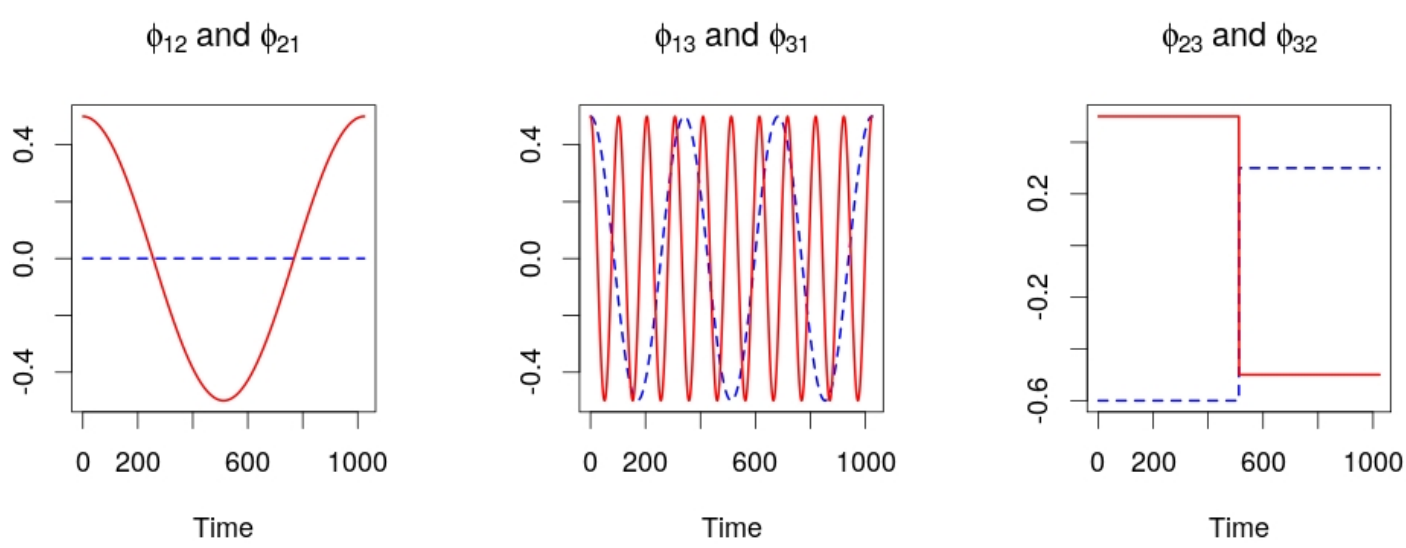

Figure 5.12. Coefficients used in Simulation 3. We created a unidirectional relation between $X_{1, t, T}$ and $X_{2, t, T}$. For $X_{1, t, T}$ and $X_{3, t, T}$ the relation is bidirectional, but with different frequencies. Finally, the relation between $X_{2, t, T}$ and $X_{3, t, T}$ is also bidirectional, with the influence $X_{2, t, T} \rightarrow X_{3, t, T}$ and $X_{2, t, T} \leftarrow X_{3, t, T}$ inverting their signs after the middle of the sampled time.

Figures A.5 to A.15 from Appendix A show the average PDC, WTC, DWC, pDWC and ppDWC for the 100 replications.

\subsection{Remarks}

PDC can identify relations within all $\mathbf{X}_{t, T}$ components. The high frequency influence caused by $\phi_{13}(u)$ appears on top-middle graph of Figure A.5 and medium frequency caused by $\phi_{31}(u)$ is 


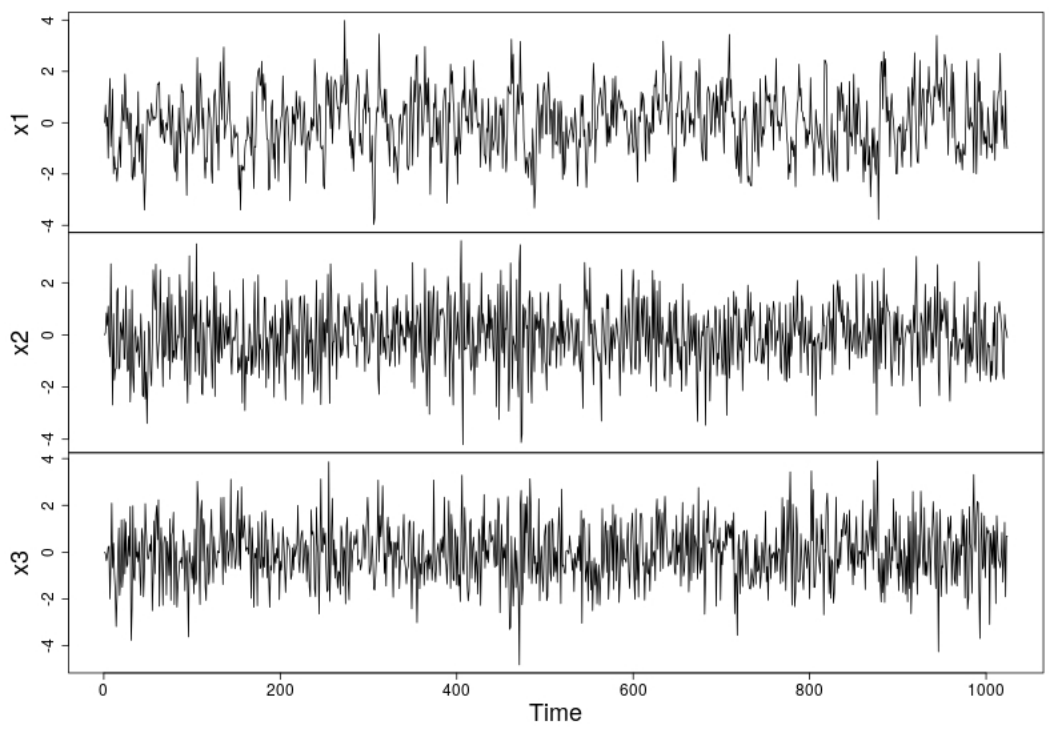

Figure 5.13. Simulation 3: Example of one simulation for process (5.3).

presented in bottom-middle graph of the same figure. Although PDC can identify directed relations between $X_{1, t, T}$ and $X_{2, t, T}$ it appears misplaced in time (top-left graph, Figure A.5), which can be due to the chosen window ( $\mathrm{M}=256$ ). For relations $X_{2, t, T} \leftrightarrow X_{3, t, T}$, PDC could not capture the sign inversion due to the fact that it is a complex-valued measure and we plot only its norm.

For DWC and pDWC, except for the high-frequency influence from $\phi_{13}(u)$ the simulated relation between the component is very evident on their graphs (Figures A.7, A.8 and A.9 for DWC and Figures A.10, A.11 and A.12 for pDWC). These two quantities are very similar and produce graphs almost identical, suggesting the linear filtering might not be effective. ppDWC also can identify the relations as DWC and pDWC do, but its curves are less smoother than DWC and pDWC. ppDWC has several quantities to be chosen: the bandwidth for Epanechnikov kernel, the tv-VAR window size, the wavelet filter, the VAR order. Moreover, we have several coefficients for the tv-VAR to estimate and the estimation of the covariance function, which is biased.

Average WTC has proven to be difficult to interpret and the conclusions are not direct and intuitive. Since it has significance test and phase between the two studied signals, this quantity shows a very detailed and local causality output which is not graphically evident for this simulation.

For all Simulations 1 to 3, we also have calculated the standard deviation for DWC, pDWC and ppDWC considering the 100 replications of each case. The graphs for these standard deviations are presented in Figures A.16 to A.26, in Appendix A. Notice that standard deviations for DWC and pDWC are less influenced by time than the standard deviation for ppDWC. 


\section{Chapter 6}

\section{An application to EEG Data}

In this chapter we consider EEG data collected from one subject (female, 23 years-old) during imaginary movement tasks. EEG data were collected at $500 \mathrm{~Hz}$, subsampled at $60 \mathrm{~Hz}$, bandpass filtered between 1 and $30 \mathrm{~Hz}$ and standardized to zero mean and standard deviation one. Figure 6.1 presents the three signals after been subsampled, filtered and standardized.

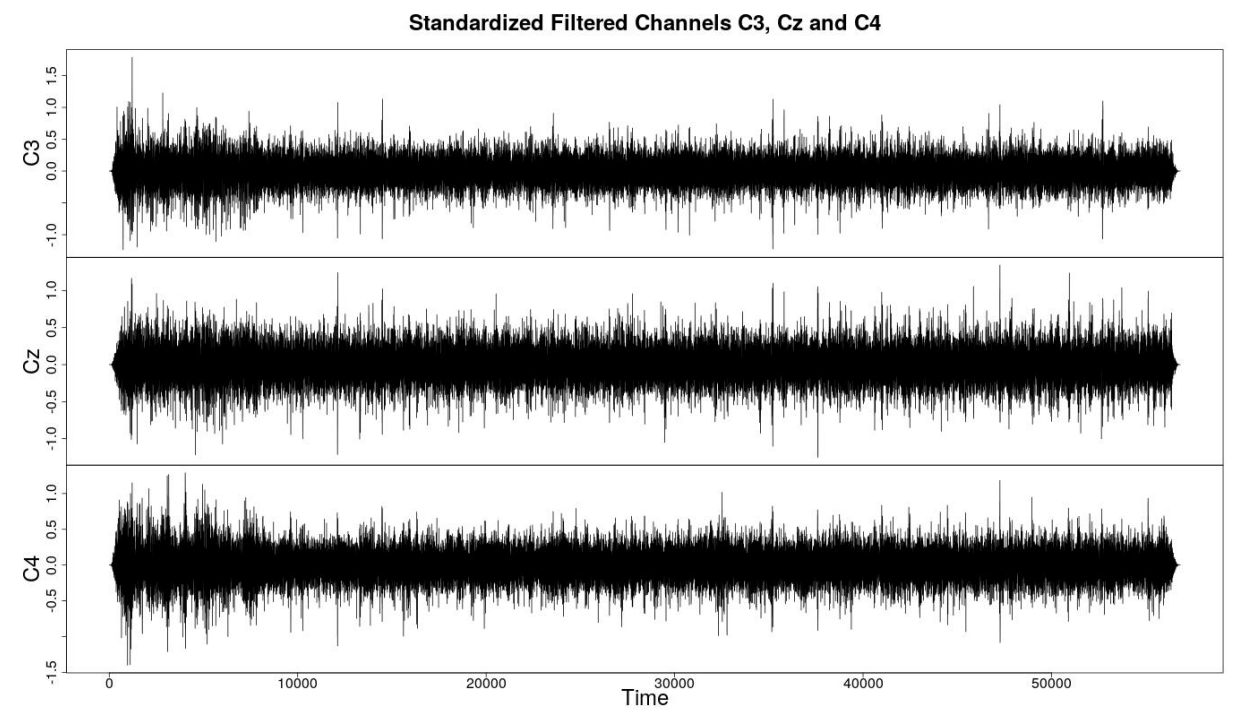

Figure 6.1. Subsampled EEG channels $C 3, C z$ and $C 4$ after been filtered and standardized.

The experiment consists of 319 trials with approximately $9000 \mathrm{~ms}$ each with the subject executing imaginary movements, described below:

- $S 200$ (80 trials): subject waits for instructions to begin the imaginary movement;

- $S 1$ (40 trials): subject is requested to begin the imaginary movement with the left hand;

- $S 10$ (40 trials): subject is requested to keep the imaginary movement with the left hand;

- $S 2$ (40 trials): subject is requested to begin the imaginary movement with the right hand;

- $S 20$ (40 trials): subject is requested to keep the imaginary movement with the right hand;

- $S 50$ (79 trials): subject can relax and waits until next set of trials. 
Figure 6.2 presents an illustration of the process.

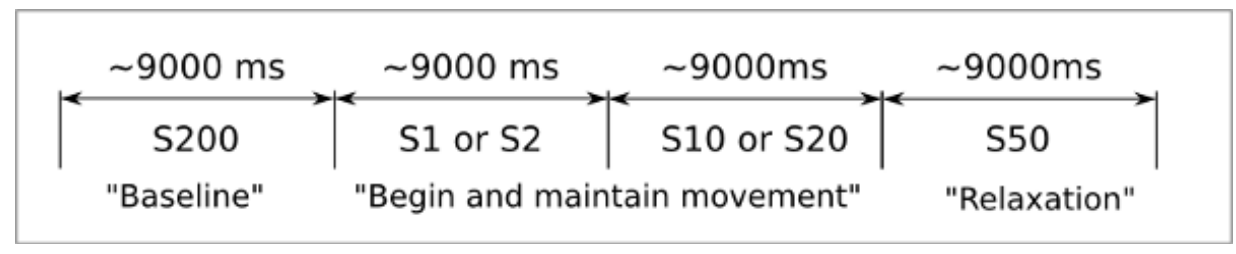

Figure 6.2. Illustration of the experiment: Before the subject starts the movements, a mark for she to get ready appears (S200). Then, the subject is requested to start an imaginary movement with one of the hands (randomly selected, S1 for left hand or S2 for right hand). The third trial of the set is to maintain the movement until the last mark disappears (S10 or S20). A period of relaxation (S50) is given between the end of this third trial and the next $\mathbf{S 2 0 0}$ that starts a new set of trials.

\subsection{DWC on EEG data}

We analyzed the data for channels $\mathrm{C} 3, \mathrm{C} 4$ and $\mathrm{Cz}$ (Figure 6.3) according to the requested task: Baseline (S200); imaginary movement with the left hand: S1 and S10 grouped; and imaginary movement with the right hand: S2 and S20 grouped.

Now, we have shown in Chapter 3, Example 3.1, that the DWC of a bivariate stationary VAR(1) is $F_{X Y, t}\left(\tau_{j}\right)=B_{X Y, t}\left(\tau_{j}\right)=0$ for any scale and time. Therefore we are interested now on testing the hypotheses $H_{0 F}: F_{X Y, t}\left(\tau_{j}\right)=0$ and $H_{0 B}: B_{X Y, t}\left(\tau_{j}\right)=0$, i.e., we want to test if the EEG's DWC has the same behavior of the DWC of a stationary VAR(1) with independent components.

Thus, for each type of trial - Baseline, Left movements and Right movements - we proceeded as follows:

- We estimated the Directed Wavelet Covariance (DWC) by trial;

- For every time and scale $\tau_{j}$, we calculate the average of $F_{X Y, t}\left(\tau_{j}\right)$ and $B_{X Y, t}\left(\tau_{j}\right), \bar{F}_{X Y, t}\left(\tau_{j}\right)$ using all trials of each type (40 observations for each type);

- Using the Central Limit Theorem, we assume normality of $\bar{F}_{X Y, t}\left(\tau_{j}\right)$ and $\bar{B}_{X Y, t}\left(\tau_{j}\right)$ for every time and scale of each trial type. We compared $\bar{F}_{X Y, t}\left(\tau_{j}\right)$ and $\bar{B}_{X Y, t}\left(\tau_{j}\right)$ with the theoretical $F_{X Y, t}\left(\tau_{j}\right)$ and $B_{X Y, t}\left(\tau_{j}\right)$ of the bivariate stationary $\operatorname{VAR}(1)$ with independent components, i.e. $F_{X Y, t}\left(\tau_{j}\right)=B_{X Y, t}\left(\tau_{j}\right)=0$.

Because the trials are collected from the same individual, in the same day and are lined up in time, it might not be valid that the trials are uncorrelated. However, given the distance between trials of same type (see Figure 6.2) and the transformation to the wavelet domain, that correlation becames weak.

Since wavelet analysis allows us to index the Directed Wavelet Covariance (DWC) in the timescale domain, it is possible to identify the directed covariances in both of these axes. The smoother scales are considered as long-term, that identify persistent influences from one channel to another. Therefore, the detail scales point the short-term variations in the directed covariance. Tables 6.1, 6.2, 


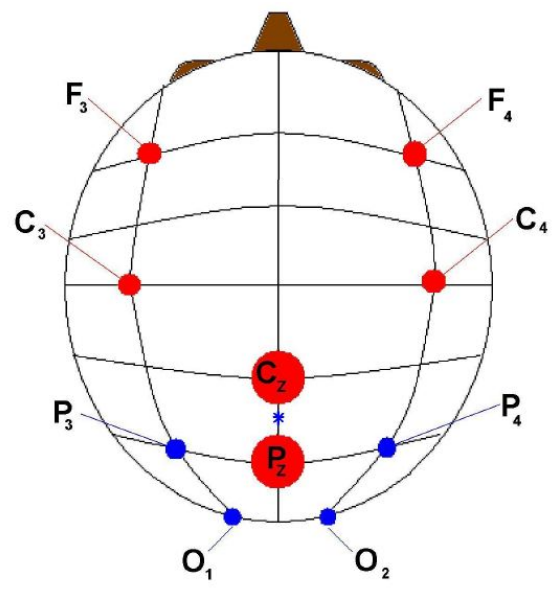

Figure 6.3. Position of EEG channels. In this chapter we use channels $C 3, C z$ and $C 4$.

and 6.3 show the proportion of rejected tests for scale versus period: begin, middle and end. Each of these periods has $1 / 3$ of the scale's observations. Figure 6.4 shows the compilation of all results separated by time (categorized) and type of relation: short (levels 1 and 2) or long-term (levels 6 and 7). Figure 6.4 shows the relations that have more than $75 \%$ of rejected tests in the period versus

\begin{tabular}{|c|c|c|c|c|c|c|c|c|c|c|c|}
\hline \multirow[b]{2}{*}{ Trial } & \multirow[b]{2}{*}{ Level } & \multirow[b]{2}{*}{$\mathrm{F} / \mathrm{B}$} & \multicolumn{3}{|c|}{$\begin{array}{c}\text { Begin } \\
\text { channels }\end{array}$} & \multicolumn{3}{|c|}{$\begin{array}{l}\text { Middle } \\
\text { channels }\end{array}$} & \multicolumn{3}{|c|}{$\begin{array}{c}\text { End } \\
\text { channels }\end{array}$} \\
\hline & & & $\begin{array}{l}\text { C3.C4 } \\
\text { sum }\end{array}$ & $\begin{array}{l}\mathrm{C} 3 . \mathrm{Cz} \\
\text { sum }\end{array}$ & $\begin{array}{c}\mathrm{C} 4 . \mathrm{Cz} \\
\text { sum }\end{array}$ & $\begin{array}{c}\text { C3.C4 } \\
\text { sum }\end{array}$ & $\begin{array}{c}\mathrm{C} 3 . \mathrm{Cz} \\
\text { sum }\end{array}$ & $\begin{array}{c}\mathrm{C} 4 . \mathrm{Cz} \\
\text { sum }\end{array}$ & $\begin{array}{c}\text { C3.C4 } \\
\text { sum }\end{array}$ & $\begin{array}{c}\mathrm{C} 3 . \mathrm{Cz} \\
\text { sum }\end{array}$ & $\begin{array}{c}\mathrm{C} 4 . \mathrm{Cz} \\
\text { sum }\end{array}$ \\
\hline \multirow[t]{14}{*}{ Rest } & 1 & $\mathrm{~F}$ & 0.00 & 1.00 & 1.00 & 0.00 & 1.00 & 1.00 & 0.00 & 0.98 & 0.98 \\
\hline & & B & 0.00 & 1.00 & 1.00 & 0.00 & 1.00 & 1.00 & 0.00 & 0.98 & 0.98 \\
\hline & 2 & $\mathrm{~F}$ & 1.00 & 0.00 & 0.00 & 1.00 & 0.00 & 0.00 & 1.00 & 0.00 & 0.00 \\
\hline & & B & 0.82 & 0.00 & 0.00 & 0.60 & 0.00 & 0.02 & 1.00 & 0.00 & 0.00 \\
\hline & 3 & $\mathrm{~F}$ & 0.00 & 1.00 & 0.47 & 0.00 & 1.00 & 1.00 & 0.00 & 0.98 & 0.05 \\
\hline & & B & 0.00 & 0.71 & 0.46 & 0.00 & 0.32 & 0.27 & 0.14 & 0.36 & 0.59 \\
\hline & 4 & $\mathrm{~F}$ & 0.00 & 1.00 & 1.00 & 0.00 & 1.00 & 1.00 & 0.00 & 1.00 & 1.00 \\
\hline & & B & 0.30 & 1.00 & 1.00 & 0.00 & 1.00 & 1.00 & 0.00 & 1.00 & 1.00 \\
\hline & 5 & F & 1.00 & 1.00 & 1.00 & 0.18 & 1.00 & 1.00 & 0.98 & 0.98 & 0.98 \\
\hline & & B & 0.37 & 1.00 & 1.00 & 0.00 & 1.00 & 1.00 & 0.78 & 0.98 & 0.98 \\
\hline & 6 & F & 0.17 & 0.00 & 0.00 & 0.10 & 0.12 & 0.00 & 0.00 & 0.95 & 0.00 \\
\hline & & B & 0.00 & 0.00 & 0.10 & 0.00 & 0.72 & 0.00 & 0.20 & 1.00 & 0.68 \\
\hline & 7 & $\mathrm{~F}$ & 0.00 & 0.00 & 0.00 & 0.00 & 0.26 & 0.00 & 0.00 & 0.32 & 0.00 \\
\hline & & B & 0.00 & 0.00 & 0.00 & 0.00 & 0.00 & 0.00 & 0.00 & 0.00 & 0.00 \\
\hline
\end{tabular}

Table 6.1. For each trial, pair of channels, and period of time we tested the hypotheses $H_{0 F}: F_{X Y, t}\left(\tau_{j}\right)=0$ and $H_{0 B}: B_{X Y, t}\left(\tau_{j}\right)=0$, i.e. there is no directed (forward or backward) relation between the channels. We present in this table the proportion of rejected tests for each period, scale and comparison for average of baseline trials (S200) using DWC.

scale, according to each type of activity the subject executed. In other words, for each type of trial, period and scale, we highlighted the periods that have the average DWC different from zero in more than $75 \%$ of the sampled time.

We want to identify in which channels relations we have $\mathrm{S} 1+\mathrm{S} 10$ or $\mathrm{S} 2+\mathrm{S} 20$ behavior different from 


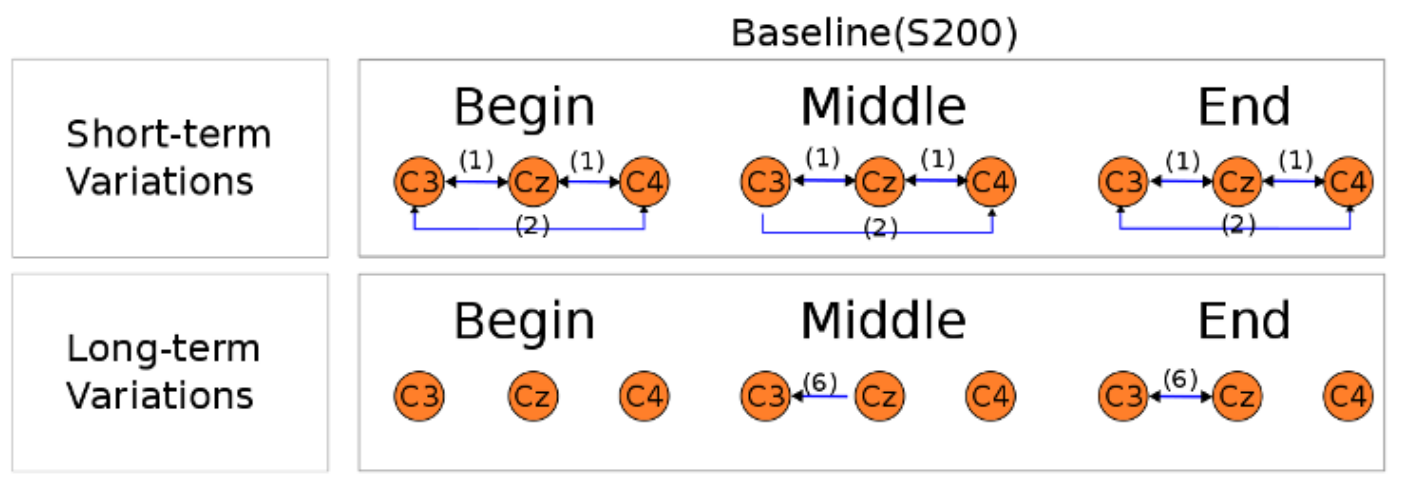

Right Movement(S2+S20)

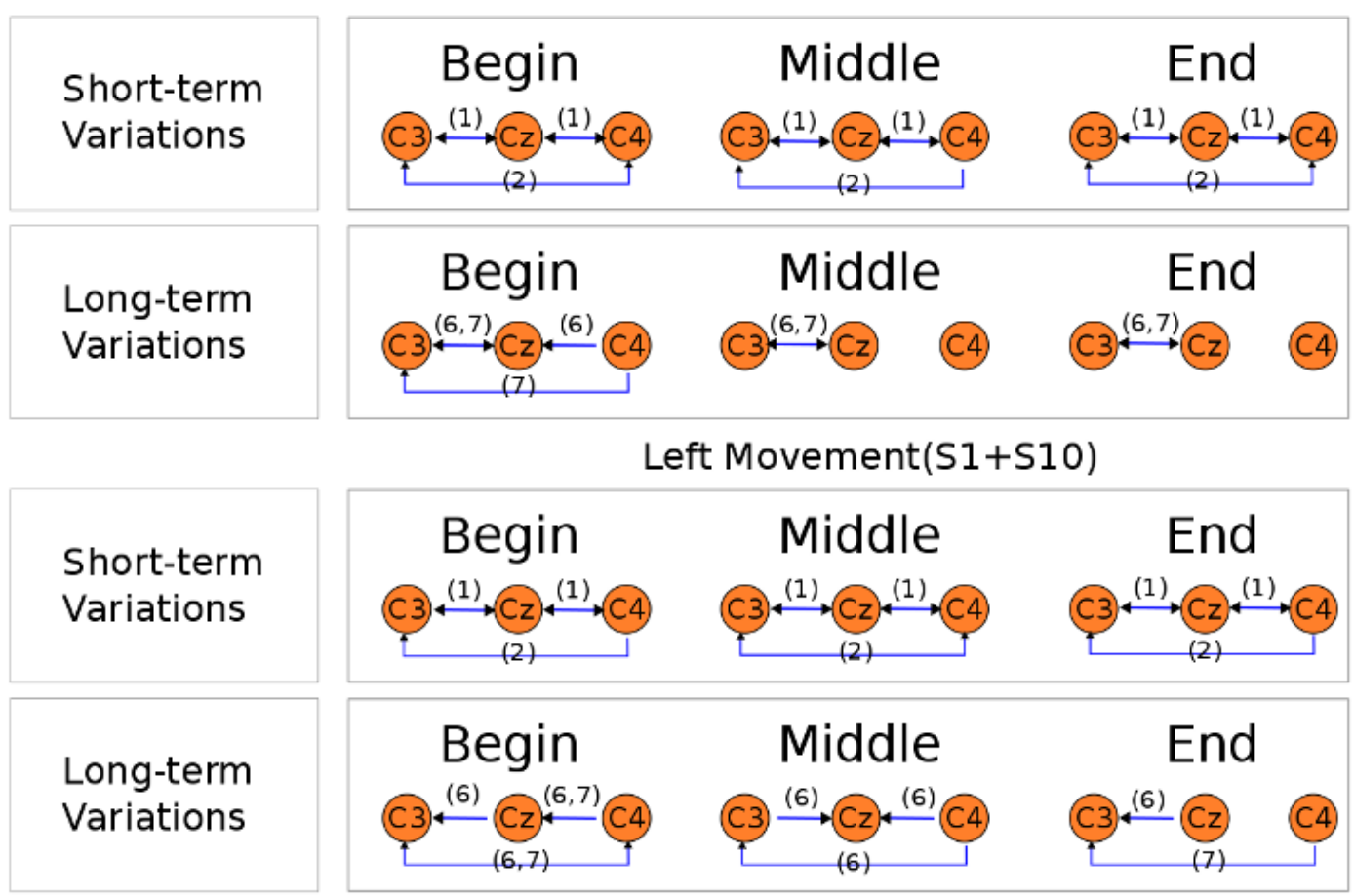

Figure 6.4. Results for DWC in EEG data by type of trials, time and scale. Begin, Middle and End are categories of time based on 1/3 of the observed interval. The number in parentheses represents in which scale the relation was found. Short-term relations were defined using levels 1 and 2 and long-term relations with levels 6 and 7 of $F_{X Y, t}\left(\tau_{j}\right)$ and $B_{X Y, t}\left(\tau_{j}\right)$. We pointed in this graph only the relations with more than $75 \%$ of rejected tests by period and scale.

baseline (S200). Thus, Figure 6.5 shows the comparison between types of trials, i.e., we drawn only the arrows of Figure 6.4 that differ between S200 and S1+S10 or between S200 and S2+S20.

Baseline trials have high activity in short-term variation and almost none in long-term (Figure 6.4). For short-term variations, there is strong energy for both directions between $\mathrm{C} 3$ and $\mathrm{C} 4$ at the beginning and at the end the period. However, for the middle period, the relation $\mathrm{C} 3-\mathrm{C} 4$ is unilateral, from C3 to C4 (Figure 6.4).

Right movement average trial shows more activity than baseline at the beginning (long-term variations) and in the middle of the period (short-term variations). At the beginning of the period, $\mathrm{C} 4$ sends more information directly to $\mathrm{C} 3$, or indirectly through $\mathrm{Cz}$ (long-term variations, see Figure 6.5). 


\begin{tabular}{|c|c|c|c|c|c|c|c|c|c|c|c|}
\hline \multirow[b]{2}{*}{ Trial } & \multirow[b]{2}{*}{ Level } & \multirow[b]{2}{*}{$\mathrm{F} / \mathrm{B}$} & \multicolumn{3}{|c|}{$\begin{array}{c}\text { Begin } \\
\text { channels }\end{array}$} & \multicolumn{3}{|c|}{$\begin{array}{l}\text { Middle } \\
\text { channels }\end{array}$} & \multicolumn{3}{|c|}{$\begin{array}{c}\text { End } \\
\text { channels }\end{array}$} \\
\hline & & & $\begin{array}{c}\text { C3.C4 } \\
\text { sum }\end{array}$ & $\begin{array}{l}\mathrm{C} 3 . \mathrm{Cz} \\
\text { sum }\end{array}$ & $\begin{array}{l}\mathrm{C} 4 . \mathrm{Cz} \\
\text { sum }\end{array}$ & $\begin{array}{l}\text { C3.C4 } \\
\text { sum }\end{array}$ & $\begin{array}{l}\mathrm{C} 3 . \mathrm{Cz} \\
\text { sum }\end{array}$ & $\begin{array}{c}\mathrm{C} 4 . \mathrm{Cz} \\
\text { sum }\end{array}$ & $\begin{array}{c}\text { C3.C4 } \\
\text { sum }\end{array}$ & $\begin{array}{c}\mathrm{C} 3 . \mathrm{Cz} \\
\text { sum }\end{array}$ & $\begin{array}{c}\mathrm{C} 4 . \mathrm{Cz} \\
\text { sum }\end{array}$ \\
\hline \multirow[t]{14}{*}{ Right } & 1 & $\mathrm{~F}$ & 0.07 & 1.00 & 1.00 & 0.34 & 1.00 & 1.00 & 0.26 & 0.99 & 0.99 \\
\hline & & B & 0.00 & 1.00 & 1.00 & 0.07 & 1.00 & 1.00 & 0.18 & 0.99 & 0.99 \\
\hline & 2 & $\mathrm{~F}$ & 0.79 & 0.00 & 0.00 & 0.17 & 0.20 & 0.00 & 0.72 & 0.30 & 0.00 \\
\hline & & B & 1.00 & 0.00 & 0.12 & 1.00 & 0.00 & 0.00 & 0.76 & 0.00 & 0.00 \\
\hline & 3 & $\mathrm{~F}$ & 0.31 & 1.00 & 0.00 & 0.23 & 1.00 & 0.06 & 0.18 & 0.99 & 0.27 \\
\hline & & B & 0.23 & 0.00 & 0.81 & 0.05 & 0.25 & 0.82 & 0.00 & 0.76 & 0.91 \\
\hline & 4 & $\mathrm{~F}$ & 0.41 & 1.00 & 1.00 & 0.49 & 1.00 & 1.00 & 0.02 & 1.00 & 1.00 \\
\hline & & B & 0.24 & 1.00 & 1.00 & 0.00 & 1.00 & 1.00 & 0.00 & 1.00 & 1.00 \\
\hline & 5 & $\mathrm{~F}$ & 0.68 & 1.00 & 1.00 & 0.09 & 0.95 & 1.00 & 0.00 & 0.99 & 0.76 \\
\hline & & B & 0.76 & 1.00 & 1.00 & 0.76 & 1.00 & 1.00 & 0.00 & 0.99 & 0.99 \\
\hline & 6 & $\mathrm{~F}$ & 0.00 & 0.00 & 0.74 & 0.00 & 0.00 & 0.42 & 0.49 & 0.00 & 0.12 \\
\hline & & B & 0.51 & 0.91 & 0.00 & 0.52 & 1.00 & 0.30 & 0.00 & 1.00 & 0.68 \\
\hline & 7 & $\mathrm{~F}$ & 0.00 & 1.00 & 0.00 & 0.00 & 1.00 & 0.00 & 0.00 & 0.98 & 0.00 \\
\hline & & B & 0.73 & 0.62 & 0.00 & 0.33 & 0.02 & 0.00 & 0.21 & 0.19 & 0.00 \\
\hline
\end{tabular}

Table 6.2. For each trial, pair of channels, and period of time we tested the hypotheses $H_{0 F}: F_{X Y, t}\left(\tau_{j}\right)=0$ and $H_{0 B}: B_{X Y, t}\left(\tau_{j}\right)=0$, i.e. there is no directed (forward or backward) relation between the channels. We present in this table the proportion of rejected tests for each period, scale and comparison for average of right movement trials $(S 2+S 20)$ using $D W C$.

\begin{tabular}{|c|c|c|c|c|c|c|c|c|c|c|c|}
\hline \multirow[b]{2}{*}{ Trial } & \multirow[b]{2}{*}{ Level } & \multirow[b]{2}{*}{$\mathrm{F} / \mathrm{B}$} & \multicolumn{3}{|c|}{$\begin{array}{c}\text { Begin } \\
\text { channels }\end{array}$} & \multicolumn{3}{|c|}{$\begin{array}{l}\text { Middle } \\
\text { channels }\end{array}$} & \multicolumn{3}{|c|}{$\begin{array}{c}\text { End } \\
\text { channels }\end{array}$} \\
\hline & & & $\begin{array}{c}\text { C3.C4 } \\
\text { sum }\end{array}$ & $\begin{array}{c}\mathrm{C} 3 . \mathrm{Cz} \\
\text { sum }\end{array}$ & $\begin{array}{c}\mathrm{C} 4 . \mathrm{Cz} \\
\text { sum }\end{array}$ & $\begin{array}{l}\text { C3.C4 } \\
\text { sum }\end{array}$ & $\begin{array}{l}\text { C3.Cz } \\
\text { sum }\end{array}$ & $\begin{array}{c}\mathrm{C} 4 . \mathrm{Cz} \\
\text { sum }\end{array}$ & $\begin{array}{c}\text { C3.C4 } \\
\text { sum }\end{array}$ & $\begin{array}{c}\mathrm{C} 3 . \mathrm{Cz} \\
\text { sum }\end{array}$ & $\begin{array}{c}\mathrm{C} 4 . \mathrm{Cz} \\
\text { sum }\end{array}$ \\
\hline \multirow[t]{14}{*}{ Left } & 1 & $\mathrm{~F}$ & 0.28 & 1.00 & 1.00 & 0.42 & 1.00 & 1.00 & 0.17 & 0.99 & 0.99 \\
\hline & & B & 0.00 & 1.00 & 1.00 & 0.40 & 1.00 & 1.00 & 0.00 & 0.99 & 0.99 \\
\hline & 2 & $\mathrm{~F}$ & 0.52 & 0.22 & 0.00 & 1.00 & 0.00 & 0.45 & 0.42 & 0.15 & 0.12 \\
\hline & & B & 0.74 & 0.35 & 0.04 & 1.00 & 0.06 & 0.42 & 1.00 & 0.00 & 0.24 \\
\hline & 3 & $\mathrm{~F}$ & 1.00 & 1.00 & 0.00 & 0.12 & 1.00 & 0.59 & 0.00 & 0.99 & 0.78 \\
\hline & & B & 0.75 & 0.08 & 1.00 & 0.23 & 0.81 & 1.00 & 0.00 & 0.99 & 0.86 \\
\hline & 4 & $\mathrm{~F}$ & 0.60 & 1.00 & 0.89 & 0.66 & 1.00 & 1.00 & 0.00 & 1.00 & 1.00 \\
\hline & & B & 0.00 & 1.00 & 1.00 & 0.49 & 1.00 & 1.00 & 0.00 & 1.00 & 0.89 \\
\hline & 5 & $\mathrm{~F}$ & 0.36 & 1.00 & 1.00 & 1.00 & 1.00 & 1.00 & 0.23 & 0.99 & 0.71 \\
\hline & & B & 0.95 & 1.00 & 1.00 & 0.96 & 1.00 & 1.00 & 0.34 & 0.99 & 0.99 \\
\hline & 6 & $\mathrm{~F}$ & 0.00 & 0.01 & 1.00 & 0.00 & 0.81 & 1.00 & 0.00 & 0.00 & 0.38 \\
\hline & & B & 0.80 & 0.45 & 0.00 & 1.00 & 0.38 & 0.00 & 0.19 & 1.00 & 0.26 \\
\hline & 7 & $\mathrm{~F}$ & 0.75 & 0.52 & 0.92 & 0.23 & 0.00 & 0.44 & 0.00 & 0.58 & 0.15 \\
\hline & & B & 0.50 & 0.00 & 0.56 & 0.38 & 0.00 & 0.54 & 0.94 & 0.44 & 0.00 \\
\hline
\end{tabular}

Table 6.3. For each trial, pair of channels, and period of time we tested the hypotheses $H_{0 F}: F_{X Y, t}\left(\tau_{j}\right)=0$ and $H_{0 B}: B_{X Y, t}\left(\tau_{j}\right)=0$, i.e. there is no directed (forward or backward) relation between the channels. We present in this table the proportion of rejected tests for each period, scale and comparison for average of left movement trials $(S 1+S 10)$ using $D W C$. 
Right Movement(S2+S20)

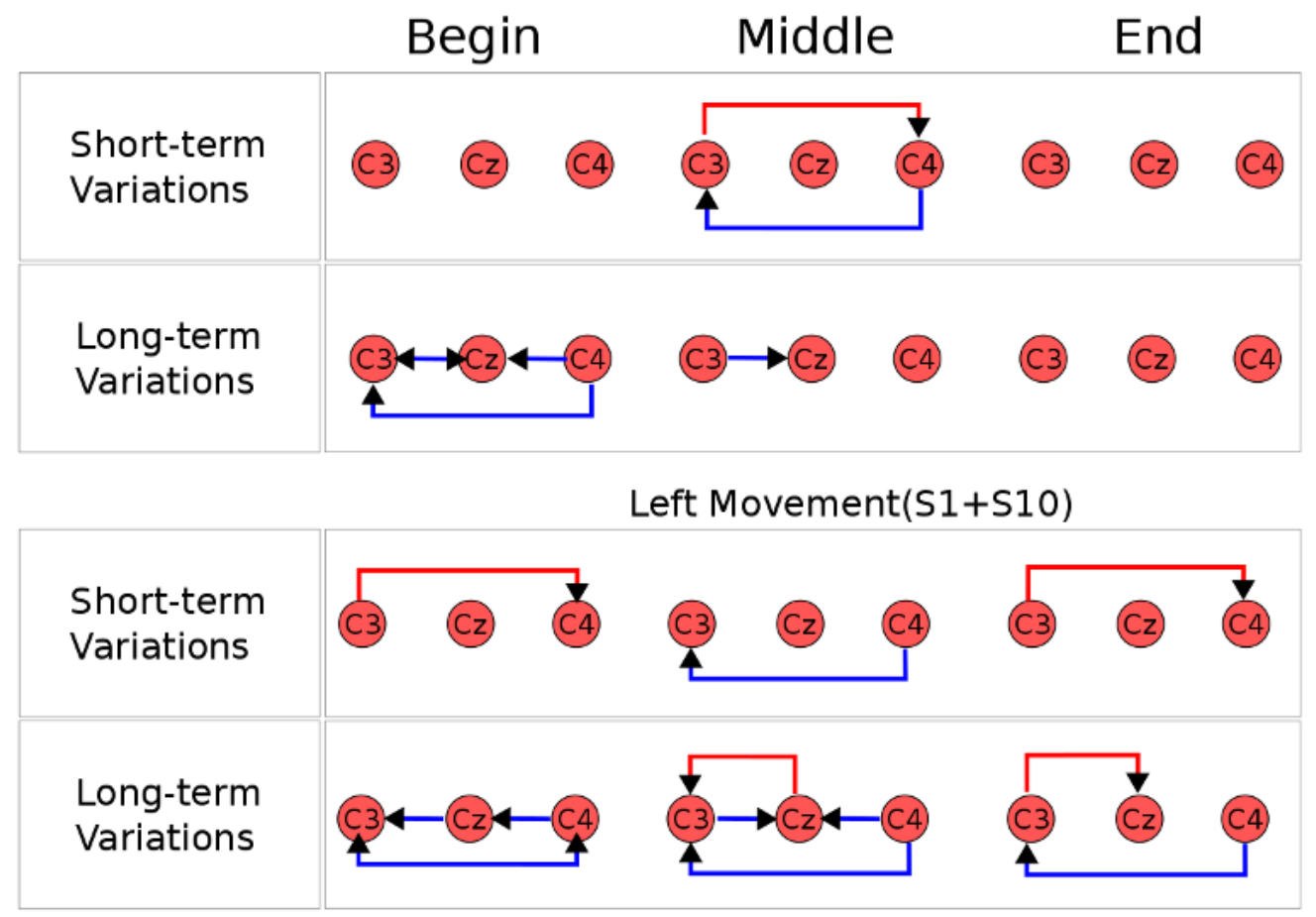

Figure 6.5. This figure shows blue arrows (arrows below or in line with channels) as the relevant relations that appear in $(S 1+S 10)$ or $(S 2+S 20)$ and do not appear in S200. Red arrows (above the channels) represent the relations that appear in baseline trials and do not appear in $(S 1+S 10)$ or $(S 2+S 20)$.

Left movement average trial also shows more activity than baseline average trial. As in right movements, $\mathrm{C} 4$ acts as seeder, sending information to $\mathrm{C} 3$ during all period for long-term variations (blue arrows in Figure 6.5). However, for short-term variations, we identified energy variation only from $\mathrm{C} 4$ to $\mathrm{C} 3$.

In both situations - right and left trials $-\mathrm{Cz}$ works as intermediate; information passes through it to reinforce the communication between $\mathrm{C} 3$ and $\mathrm{C} 4$.

\section{2 pDWC on EEG data}

Although analyses with DWC have identified several relations between $\mathrm{C} 3, \mathrm{C} 4$ and $\mathrm{Cz}$, they do not remove influence of exogenous channels. For instance, when we use DWC to evaluate the relation between $\mathrm{C} 3$ and $\mathrm{Cz}$, we do not remove the $\mathrm{C} 4$ influence on this relation. pDWC and ppDWC, on the other hand, aim to mitigate the interference of exogenous variables on the measured relation e.g., the influence of $\mathrm{C} 4$ on relation $\mathrm{C} 3-\mathrm{Cz}$.

pDWC has the same properties of DWC. In fact, under the assumption that the process follows a stationary $\operatorname{VAR}(1)$ with independent components, $p F_{(k, m), t}\left(\tau_{j}\right)=p B_{(k, m), t}\left(\tau_{j}\right)=0, k, m=1,2,3$. Tables 6.4 to 6.6 present the analogous analyses of Section 6.1, but now using pDWC: each cell on these tables represents the proportion of rejected tests for $H_{0 F}: p F=0$ and $H_{0 B}: p B=0$ along the trials.

The conclusions in this section are different than using DWC. While DWC identified intense brain 


\begin{tabular}{llccccccccc}
\hline & S200 & \multicolumn{9}{c}{ Period } \\
Level & Direction & C3.C4 & C3.Cz & C4.Cz & C3.C4 & C3.Cz & C4.Cz & C3.C4 & C3.Cz & C4.Cz \\
\hline 1 & F & 0.00 & 0.00 & 0.89 & 0.00 & 0.00 & 0.10 & 0.00 & 0.00 & 0.28 \\
& B & 0.00 & 0.00 & 1.00 & 0.00 & 0.00 & 0.38 & 0.00 & 0.00 & 0.77 \\
2 & F & 0.07 & 0.00 & 0.00 & 0.02 & 0.00 & 0.00 & 0.00 & 0.00 & 0.00 \\
& B & 0.00 & 0.00 & 0.05 & 0.00 & 0.00 & 0.00 & 0.00 & 0.00 & 0.00 \\
3 & F & 0.00 & 0.00 & 0.00 & 0.00 & 0.00 & 0.05 & 0.00 & 0.00 & 0.00 \\
& B & 0.00 & 0.00 & 0.14 & 0.00 & 0.00 & 0.22 & 0.00 & 0.00 & 0.00 \\
4 & F & 0.00 & 0.00 & 0.77 & 0.00 & 0.00 & 0.05 & 0.00 & 0.00 & 0.25 \\
& B & 0.00 & 0.00 & 0.71 & 0.00 & 0.00 & 0.09 & 0.00 & 0.00 & 0.37 \\
5 & F & 0.00 & 0.00 & 0.35 & 0.00 & 0.00 & 0.00 & 0.00 & 0.00 & 0.00 \\
& B & 0.00 & 0.00 & 0.65 & 0.00 & 0.00 & 0.08 & 0.00 & 0.00 & 0.00 \\
6 & F & 0.00 & 0.00 & 0.00 & 0.00 & 0.00 & 0.00 & 0.00 & 0.00 & 0.00 \\
& B & 0.00 & 0.00 & 0.00 & 0.00 & 0.00 & 0.00 & 0.00 & 0.00 & 0.00 \\
7 & F & 0.00 & 0.00 & 0.00 & 0.00 & 0.00 & 0.00 & 0.00 & 0.00 & 0.00 \\
& B & 0.00 & 0.00 & 0.00 & 0.00 & 0.00 & 0.00 & 0.00 & 0.00 & 0.00 \\
8 & F & 0.00 & 0.00 & 0.89 & 0.00 & 0.00 & 0.10 & 0.00 & 0.00 & 0.28 \\
& B & 0.00 & 0.00 & 1.00 & 0.00 & 0.00 & 0.38 & 0.00 & 0.00 & 0.77 \\
\hline
\end{tabular}

Table 6.4. For each trial, pair of channels, and period of time we tested the hypotheses $H_{0 F}: p F=0$ and $H_{0 B}: p B=0$, i.e. there is no directed (forward or backward) relation between the channels. We present in this table the proportion of rejected tests for each period, scale and comparison for average of baseline trials (S200) using $p D W C$.

activity for these channels, pDWC shows fewer cells with high proportion of rejected tests. Relations between $\mathrm{C} 4$ and $\mathrm{Cz}$ are the only strong relations between the trials.

For baseline trials (S200 - Table 6.4), pDWC identified two strong relations: bilateral activity between $\mathrm{C} 4$ and $\mathrm{Cz}$ at the beginning of the period (scales 1, 4 and 8) and $C 4 \leftarrow C z$ at the end of the period (scales 1 and 8 ).

For right movements (trials $\mathrm{S} 2+\mathrm{S} 20$, Table 6.5), high proportions appear for $C 4 \leftrightarrow C z$ at the beginning and in the middle (scales 1, 4 and 5). At the end of the period, pDWC presented high proportion of rejected tests for $C 4 \leftrightarrow C z$ (scale 1 ) and for $C 4 \leftarrow C z$ (scale 3).

Notice that, for relation $C 3 \rightarrow C 4$ on baseline trials, we rejected $32 \%$ of the tests at the beginning, on scale 1 (Table 6.5). This means that $32 \%$ of the time on the first period, the average pDWC for baseline trials is different from zero.

For left movements (Table 6.6), we have found high activity between $\mathrm{C} 4$ and $\mathrm{Cz}$ in the entire period on scales 1, 4 and 5. Relation $C 4 \leftarrow C z$ also presented high proportion at the beginning (scale 3), middle (scale 4) and at the end of the period (scale 3).

\subsection{Remarks}

In this chapter we have shown an application for DWC and pDWC with real data. We studied EEG data from three channels of one subject during imaginary movement tasks. The studied channels were $\mathrm{C} 3, \mathrm{C} 4$ and $\mathrm{Cz}$ and types of imaginary tasks were categorized in relaxation, right movement and left movement, each one with 40 trials during the collected section. We calculated the average DWC and PDWC by type of trial and compared them with their theoretical values in a bivariate stationary 


\begin{tabular}{llccccccccc}
\hline & S2+S20 & \multicolumn{9}{c}{ Period } \\
Level & Direction & C3.C4 & C3.Cz & C4.Cz & C3.C4 & C3.Cz & C4.Cz & C3.C4 & C3.Cz & C4.Cz \\
\hline 1 & F & 0.32 & 0.00 & 0.94 & 0.00 & 0.00 & 0.67 & 0.02 & 0.00 & 0.73 \\
& B & 0.00 & 0.00 & 0.90 & 0.00 & 0.00 & 1.00 & 0.00 & 0.00 & 0.97 \\
2 & F & 0.00 & 0.00 & 0.30 & 0.00 & 0.00 & 0.00 & 0.00 & 0.00 & 0.00 \\
& B & 0.18 & 0.00 & 0.03 & 0.00 & 0.00 & 0.27 & 0.00 & 0.00 & 0.00 \\
3 & F & 0.02 & 0.08 & 0.00 & 0.00 & 0.00 & 0.35 & 0.00 & 0.00 & 0.00 \\
& B & 0.00 & 0.00 & 0.38 & 0.00 & 0.00 & 0.32 & 0.00 & 0.00 & 0.80 \\
4 & F & 0.05 & 0.00 & 0.91 & 0.00 & 0.00 & 0.69 & 0.00 & 0.00 & 0.41 \\
& B & 0.07 & 0.00 & 0.94 & 0.09 & 0.00 & 0.75 & 0.00 & 0.00 & 0.62 \\
5 & F & 0.00 & 0.00 & 0.68 & 0.00 & 0.00 & 0.51 & 0.00 & 0.00 & 0.00 \\
& B & 0.00 & 0.00 & 1.00 & 0.00 & 0.00 & 0.76 & 0.00 & 0.00 & 0.00 \\
6 & F & 0.00 & 0.00 & 0.09 & 0.00 & 0.00 & 0.00 & 0.00 & 0.00 & 0.00 \\
& B & 0.00 & 0.00 & 0.00 & 0.00 & 0.00 & 0.30 & 0.00 & 0.00 & 0.09 \\
7 & F & 0.00 & 0.00 & 0.27 & 0.00 & 0.00 & 0.00 & 0.00 & 0.00 & 0.00 \\
& B & 0.00 & 0.00 & 0.20 & 0.00 & 0.00 & 0.00 & 0.00 & 0.00 & 0.00 \\
8 & F & 0.00 & 0.00 & 0.00 & 0.00 & 0.00 & 0.00 & 0.00 & 0.00 & 0.00 \\
& B & 0.00 & 0.00 & 0.00 & 0.00 & 0.00 & 0.00 & 0.00 & 0.00 & 0.00 \\
\hline
\end{tabular}

Table 6.5. For each trial, pair of channels, and period of time we tested the hypotheses $H_{0 F}: p F=0$ and $H_{0 B}: p B=0$, i.e. there is no directed (forward or backward) relation between the channels. We present in this table the proportion of rejected tests for each period, scale and comparison for average of right movement trials $(S 2+S 20)$ using $p D W C$.

\begin{tabular}{llllllcccccc}
\hline & S1 + S10 & \multicolumn{9}{c}{ Period } \\
& & \multicolumn{9}{c}{ Begin } & \multicolumn{3}{c}{ End } \\
Level & Direction & C3.C4 & C3.Cz & C4.Cz & C3.C4 & C3.Cz & C4.Cz & C3.C4 & C3.Cz & C4.Cz \\
\hline 1 & F & 0.00 & 0.00 & 1.00 & 0.10 & 0.00 & 0.71 & 0.00 & 0.00 & 0.98 \\
& B & 0.17 & 0.00 & 1.00 & 0.11 & 0.00 & 0.83 & 0.00 & 0.00 & 0.98 \\
2 & F & 0.00 & 0.00 & 0.00 & 0.33 & 0.00 & 0.22 & 0.02 & 0.00 & 0.00 \\
& B & 0.00 & 0.00 & 0.00 & 0.00 & 0.00 & 0.00 & 0.00 & 0.00 & 0.07 \\
3 & F & 0.16 & 0.15 & 0.00 & 0.00 & 0.00 & 0.26 & 0.00 & 0.00 & 0.37 \\
& B & 0.02 & 0.00 & 1.00 & 0.16 & 0.15 & 0.28 & 0.00 & 0.28 & 0.60 \\
4 & F & 0.00 & 0.00 & 0.60 & 0.00 & 0.00 & 0.40 & 0.00 & 0.00 & 0.76 \\
& B & 0.00 & 0.00 & 0.65 & 0.00 & 0.00 & 0.72 & 0.00 & 0.00 & 0.53 \\
5 & F & 0.00 & 0.00 & 0.67 & 0.00 & 0.00 & 0.50 & 0.00 & 0.00 & 0.60 \\
& B & 0.00 & 0.00 & 1.00 & 0.00 & 0.00 & 0.32 & 0.00 & 0.00 & 0.49 \\
6 & F & 0.00 & 0.00 & 0.00 & 0.00 & 0.00 & 0.19 & 0.00 & 0.00 & 0.07 \\
& B & 0.00 & 0.00 & 0.00 & 0.00 & 0.00 & 0.00 & 0.00 & 0.00 & 0.00 \\
7 & F & 0.00 & 0.02 & 0.00 & 0.00 & 0.00 & 0.00 & 0.00 & 0.00 & 0.00 \\
& B & 0.00 & 0.00 & 0.31 & 0.00 & 0.00 & 0.00 & 0.00 & 0.00 & 0.00 \\
8 & F & 0.00 & 0.00 & 0.00 & 0.00 & 0.00 & 0.00 & 0.00 & 0.00 & 0.00 \\
& B & 0.00 & 0.00 & 0.00 & 0.00 & 0.00 & 0.00 & 0.00 & 0.00 & 0.00 \\
\hline
\end{tabular}

Table 6.6. For each trial, pair of channels, and period of time we tested the hypotheses $H_{0 F}: p F=0$ and $H_{0 B}: p B=0$, i.e. there is no directed (forward or backward) relation between the channels. We present in this table the proportion of rejected tests for each period, scale and comparison for average of left movement trials (S1+S10) using $p D W C$. 
VAR(1) with independent components. We presented techniques to summarize information and proposed a significance test based on Central Limit Theorem that, even assuming a weak dependency between the trials, is still valid. We finally compared different types of trials by their variations on the proportion of rejected tests, per scale and period.

Considering DWC analyses, $\mathrm{C} 4$ channel acted as seeder of information and $\mathrm{C} 3$ received information directly or through $\mathrm{Cz}$. On the other hand, $\mathrm{Cz}$ is an intermediate channel, that mainly serves to the purpose of connecting $\mathrm{C} 3$ and $\mathrm{C} 4$. From Figure 6.4 we also identified that baseline trials activities were mostly in short-term variations, and not in long-term persistent variations. For this subject, we identified more activity in left movements than in right movements.

We also presented analyses for the given EEG data using pDWC. We have seen that analyses with pDWC have found less significant activities than using DWC. Nevertheless, in these analyses we still identified that S200 trials have predominant activity in short-term variations and mainly between $\mathrm{C} 4$ and $\mathrm{Cz}$ channels. We also concluded that left movement trials have the highest activity among the three studied trials. 


\section{Chapter 7}

\section{Discussion}

We proposed three decompositions of the wavelet covariance that aim to find relations between components in a multivariate locally stationary process. Wavelet transforms better represent processes with local variations, outliers and drastic pattern changes better than Fourier transforms. They are indexed in time and have multiscale resolution, which identifies both local short-term and long persistent variations.

Results with simulated data suggested that the proposed quantities capture the causal structure between locally stationary processes. We first simulated two situations, one with drastic changes of the covariance structure and other with subtle changing of the structure, according to a cosine function. In both situations the proposed Directed Wavelet Covariance captured the simulated forward and backward relations. In a third simulation, we simulated a trivariate process with directed and time-varying influences between the components. In this case, we analyzed the quantities' effectiveness on identifying each bivariate relation excluding the outer influences of the other components.

Comparisons between Directed Wavelet Covariance (DWC), Wavelet Coherence (WTC) and Partial Directed Coherence (PDC) with moving windows have shown that all three methodologies can identify the simulated patterns, with different limitations and strengths. PDC has the additional information of which frequencies the relations appear and help to identify situations with causal relationships in unexpected frequencies. For instance, medical studies describe fundamental frequencies for physiological mechanisms like heart and respiratory frequencies. In Neurosciences context, alpha and beta frequencies during REM sleep state are well established. For instance, Ginter et al. (2005) studied beta and gamma frequencies and their characteristics in EEG signals and reported synchronized gamma frequencies during motor tasks. However, one should suppose stationarity of the processes and that we can write the multivariate analyzed process as a $\operatorname{VAR}(\mathrm{p})$ with $p$ properly chosen.

Wavelet Coherence also estimates causal relations between signals by measuring phases between wavelet coefficients and, as in Directed Wavelet Covariance, it estimates the causal relation in the time-scale domain. Nevertheless, the phases only suggest causal relations and are not related to causality concepts like Granger causality. Moreover, we can calculate these phases only when we choose a complex wavelet, like Morlet wavelet. 
The proposed Directed Wavelet Covariance (DWC) does not need the stationarity assumption. Moreover, it is suitable for locally stationary processes and, since it uses wavelet transforms, deals better with processes with local regimes, outliers and drastic pattern changes. During the simulation studies, DWC identified the covariance structures as expected and corroborated the theoretical results. However, we proposed DWC estimators using sample cross-covariance (3.3), which produces biased estimators of (3.1) and (3.2). Another issue, also related to the bias, is the necessity of choosing $b_{T}$ in (3.3). Dahlhaus (1996) has shown that if $K($.$) is the Epanechnikov kernel, the optimal b_{T}$ that minimizes the RMSE for sample cross-covariance (3.3) is:

$$
\begin{aligned}
b_{t}^{o p t}=b_{f}^{o p t} & =\left(\frac{576 \pi}{T}\right)^{1 / 6}\left(\frac{\Delta_{\lambda}}{\Delta_{u}^{5}}\right)^{1 / 12}, \\
\Delta_{u} & :=\frac{\partial^{2}}{\partial u^{2}} f(u, \lambda) / f(u, \lambda) \\
\Delta_{\lambda} & :=\frac{\partial^{2}}{\partial \lambda^{2}} f(u, \lambda) / f(u, \lambda) .
\end{aligned}
$$

Equation (7.1) suggests that $b_{t}$ is directly related with growth rate ratio between dimensions time and frequency. To calculate $b_{t}$ using quantity (7.1), we first need to estimate the evolutionary spectrum. Morettin (2014) presents several methods to estimate the evolutionary spectrum, including locally stationary processes (as defined in 2.25 ) and locally stationary wavelet processes (Nason et al. (2000)).

However, consider the model in (5.1) and simulate another 100 different time series with $\mathrm{T}=512$. Figure $7.1\left(b_{t}=0.2\right)$ and Figure $7.2\left(b_{t}=0.7\right)$ present the DWC (average $F($.$) and B($.$) using 100$ replications) with Epanechnikov kernel and different values of $b_{t}$. Notice that, despite of having a big difference from 0.2 to 0.7 on bandwidth, the DWC does not have big changes, except that using $b_{t}=0.7$ the curves are smoother, as expected. On the other hand, if we consider a linear kernel instead of Epanechnikov kernel, we have the DWC presented in Figure 7.3. Comparing this figure with Figure 7.1 we see almost no changes, except that Figure 7.1 is smoother and has a smaller scale in the vertical axis than Figure 7.3.

Another limitation of DWC is the restriction of the circularity assumption on its calculation. For levels $j<J$ with width $L_{j}=\left(2^{j}-1\right)\left(L_{1}-1\right)+1$, the first and the last $L_{j}$ coefficients must be discarded. For instance, for the Haar wavelet filter, which has width $L_{1}=2$, we have then $L_{J}=2^{J}=\mathrm{T}$ and we may not calculate the coefficients in the last scale. If $L_{1}>2$ more than only the last scale will be affected and one should deal with the trade-off: wider filter $\times$ fewer scales. On the other hand, smoother wavelets may produce more effective and precise solutions in the calculated scales. The trade-off between losing scales of analysis and using smoother wavelets must be found by the user and can vary from an application to another.

We discuss not only bivariate but also multivariate process with more than two components in 

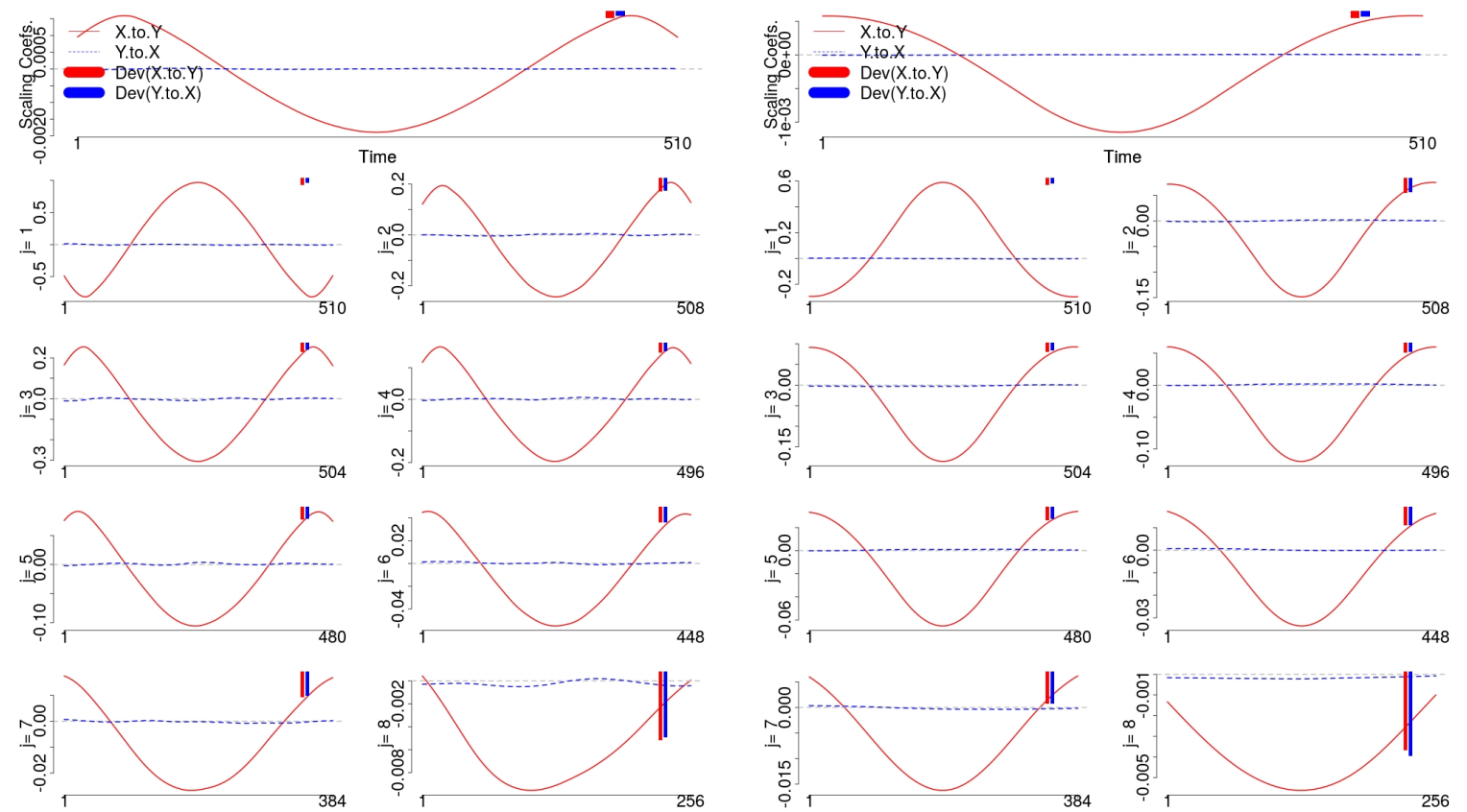

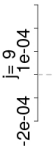

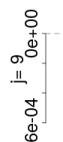

Figure 7.1. Average DWC for 100 replications of Figure 7.2. Average DWC for 100 replications of model (5.1) estimated using Haar wavelets, Epanechnikov kernel and $b_{T}=0.2$.

model (5.1) estimated using Haar wavelets, Epanechnikov kernel and $b_{T}=0.7$.
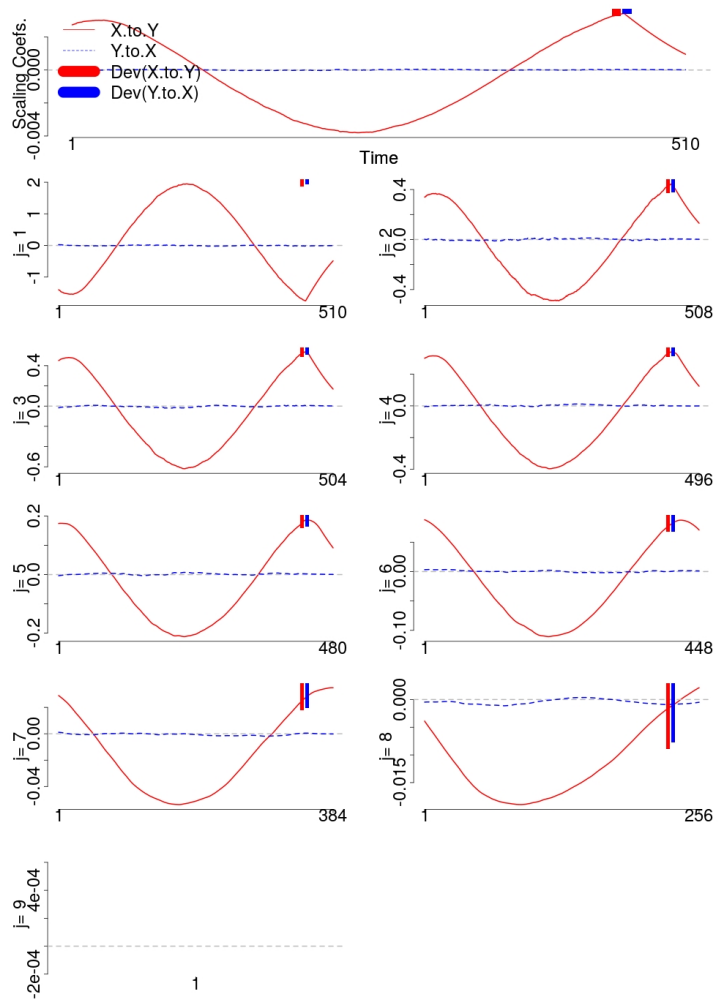

256 and $b_{T}=0.2$. 
this study. In this case, the issue is isolating the effects of exogenous components when we study a bivariate relation in a multivariate process. We proposed two quantities to deal with this situation: the Partial Directed Wavelet Covariance based on linear filters (pDWC) and the Partial Directed Wavelet Covariance based on time-varying Vector Autoregressive models (ppDWC). We have seen that pDWC has properties very similar to the DWC. Results in Simulation 3 have shown that both quantities (DWC and pDWC) produce similar results if the linear filter is ineffective. ppDWC has shown to be very sensible to the tv-VAR estimation and has expectation and variance hard to be calculated. Moreover, besides of the kernel and its bandwidth to be chosen in non-stationary crosscovariance function estimation, ppDWC also requires a window size and the order of the tv-VAR to be chosen.

Among the three proposed quantities, DWC and pDWC have shown to be more precise and reliable. For instance, in Simulation 3 we have considered a trivariate tv-VAR(1) and we have analyzed 100 replications of that process with $\mathrm{T}=1024$ time observations. DWC and pDWC have produced very similar results for this simulation. Despite of identifying the simulated relations, ppDWC produces less smooth curves (compared to those found by DWC and pDWC). Also, the direct relations are less evident and its standard deviations oscillates more in time. Still considering that simulation, PDC estimated with moving windows have identified all simulated relations between the components, although some were misplaced in time due to the choice of the window size. Is important to mention that PDC cannot identify sign inversions of the coefficients. Wavelet Coherence produced very detailed but inconclusive graphs, difficult to identify an overall pattern for the simulated data. This quantity therefore is recommended for time series whose interest is evaluating local influences between the studied data.

In Chapter 6 we presented an EEG data analysis with DWC and pDWC and they have produced different outputs with coherent results. Both analyzes identified relations between channels $\mathrm{C} 4$ and $\mathrm{Cz}$ for baseline trials in short-term variation levels. We also identified that left movements trials produced more activity between the three observed channels. In this particular application, the main difference between the two methodologies relied on the fact that DWC pointed more relations in more scales as significant, while pDWC identified only the strongest relations.

Future studies include the asymptotic distribution of the estimated DWC and significance tests for the DWC and pDWC by scale and time. We also intend to study how the choice of $b_{T}$ and the bias in (3.3) impact the DWC and pDWC estimators. ppDWC has an interesting approach and reasonable assumptions to estimate the time-dependent directed influence between process. Its concept is more sophisticated than the 'naive' linear filter proposed in pDWC. However, it is necessary a better study of its properties and probability distribution. For this quantity we intend to investigate its covariance oscillation on time and how it behaviors depending on how the tv-VAR is estimated.

In a different approach, whether considering locally stationary processes as defined in (Dahlhaus 
(1996)), we could consider locally stationary wavelet processes (Nason et al. (2000)). These processes are based on discrete, non-decimated and compactly supported wavelets and also consider rescaled time $u=t / T$ to increase the amount of information in local structures.

Another approach, possibly more suitable to image sequences, is to study the DWC using scalemixing wavelet spectra. Jeon et al. (2014) proposed a generalization of the scale-mixing wavelet spectra to the complex wavelet domain. They used this generalization to classify mammograms with suspicion of cancer, as benign or malignant. Remenyi et al. (2014) presented the scale-mixing wavelet spectra estimation using empirical Bayesian techniques. In this context, we intend to study the scale-mixing wavelet spectra in terms of forward and backward signals and how to propose a decomposition like the DWC using these concepts.

The proposed Directed Wavelet Covariance decomposition is a different approach to study causality in non-stationary processes. The use of wavelets is a gain and adds to the number of studies that can be addressed when Fourier transform does not apply. The pDWC is an alternative for multivariate processes and it removes linear influences from observed external components. 


\section{Appendix A}

\section{Figures}

$F: I V=1$

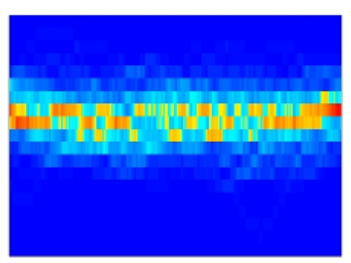

$F: I V=5$

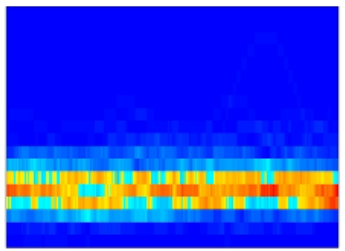

$B: I V=1$

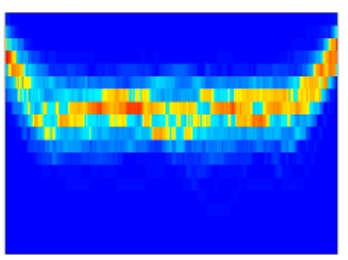

$B: I v=5$

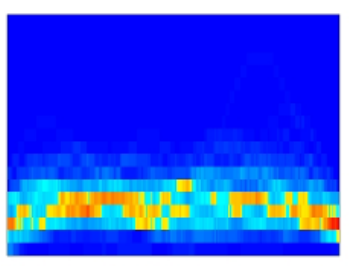

$F: I V=2$

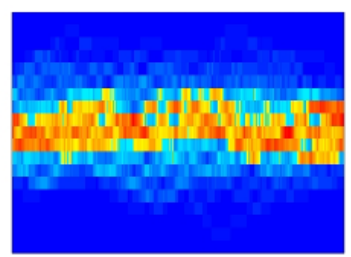

$F: I V=6$

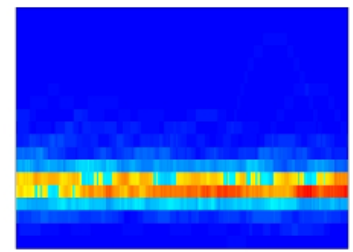

$B: I V=2$

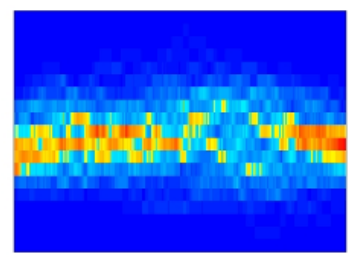

$B: l v=6$

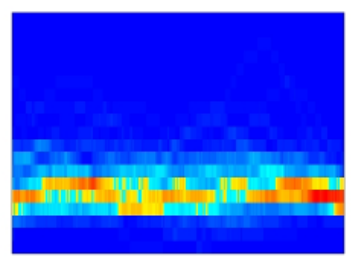

$F: I V=3$

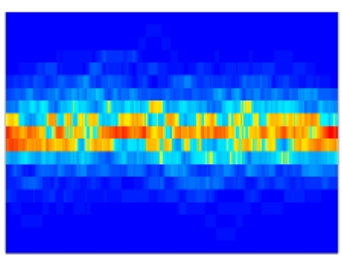

$F: I V=7$

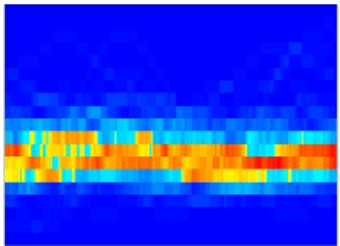

$B: I V=3$

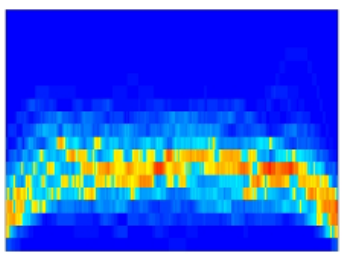

$B: I v=7$

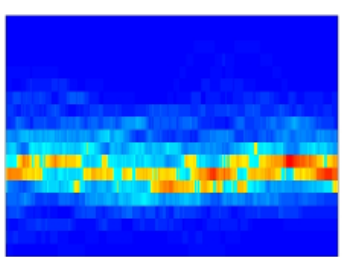

$F: I V=4$

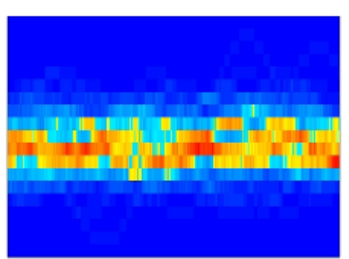

$F: I V=8$

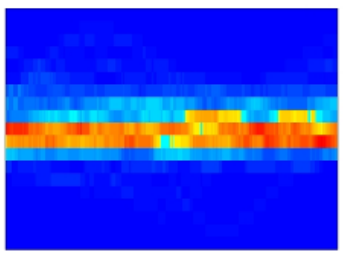

$B: I v=4$

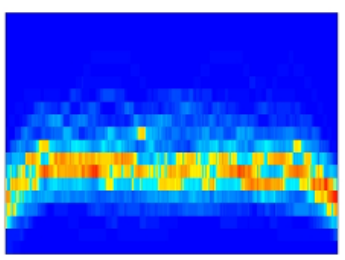

$B: l v=8$

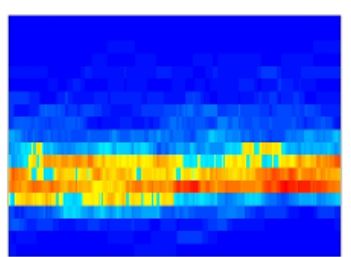

Figure A.1. Empirical Distribution for $F_{12, t}\left(\tau_{j}\right)$ and $B_{12, t}\left(\tau_{j}\right)$ using 100 replications of the process presented in Example 3.2. 
$F: I V=1$

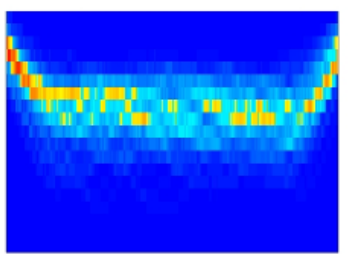

$F: I V=5$

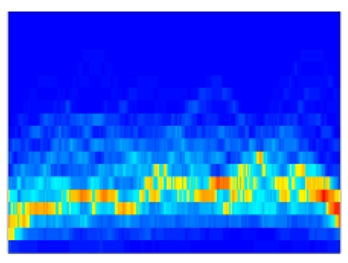

$B: I v=1$

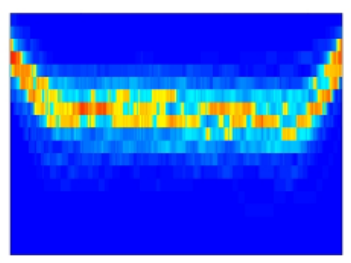

$B: I v=5$

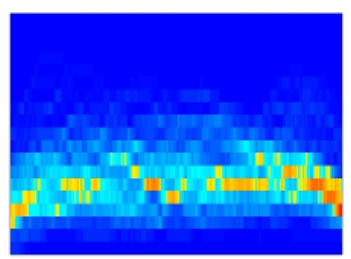

$F: I V=2$

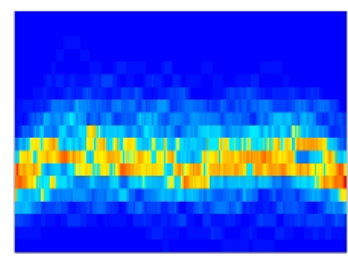

$F: I V=6$

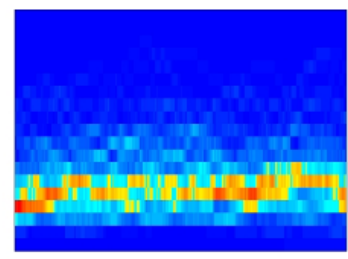

$B: l v=2$

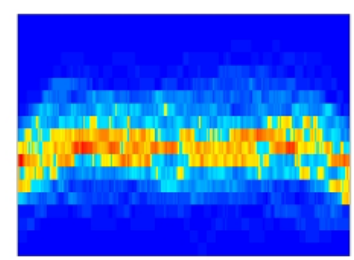

$B: I v=6$

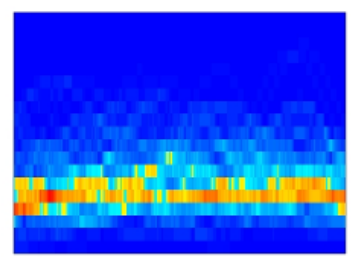

$F: I V=3$

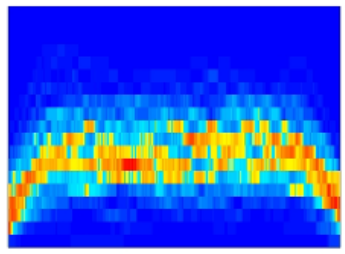

$F: I v=7$

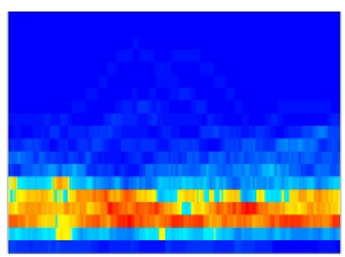

$B: I v=3$

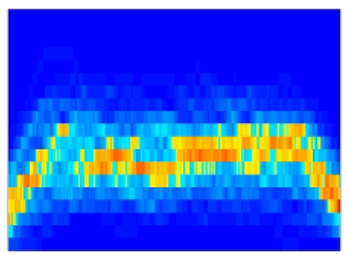

$B: I v=7$

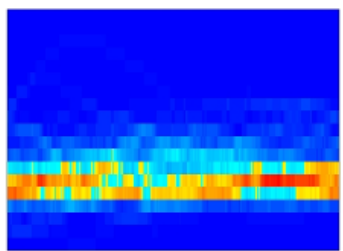

$F: I V=4$

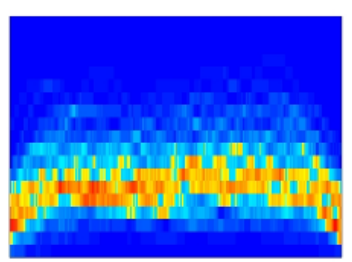

$F: I V=8$

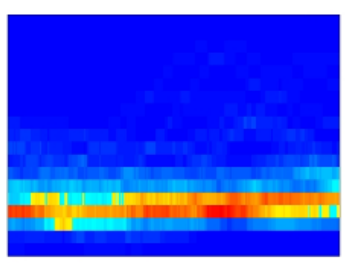

$B: l v=4$

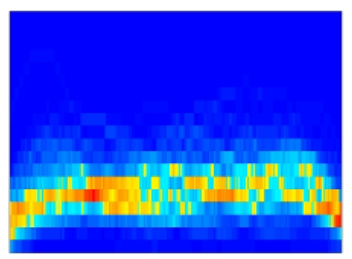

$B: I v=8$

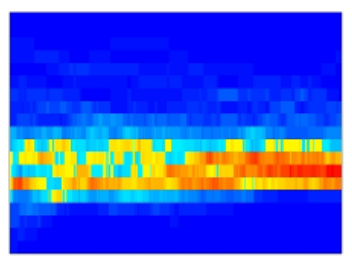

Figure A.2. Empirical Distribution for $F_{12, t}\left(\tau_{j}\right)$ and $B_{12, t}\left(\tau_{j}\right)$ using 100 replications of the process presented in Example 3.3 with $a=b=0.6$. 
$F: I V=1$

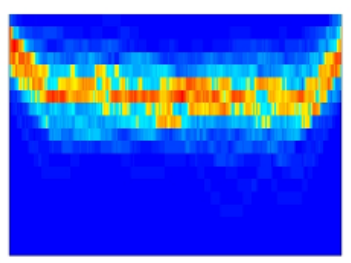

$F: I V=5$

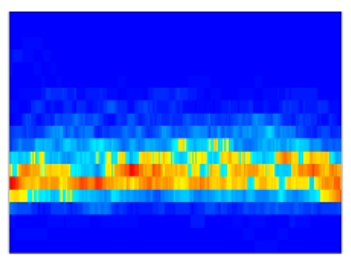

$B: I v=1$

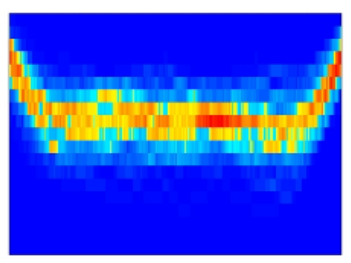

$B: I v=5$

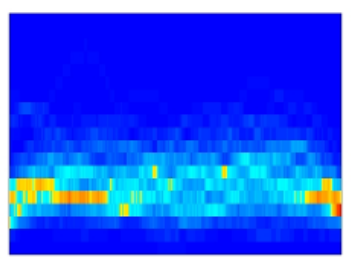

$F: I V=2$

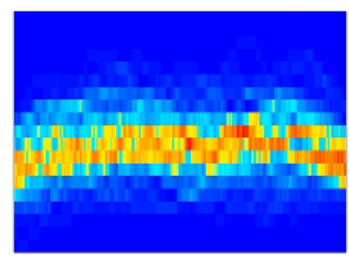

$F: I V=6$

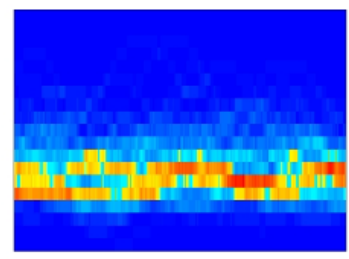

$B: I v=2$

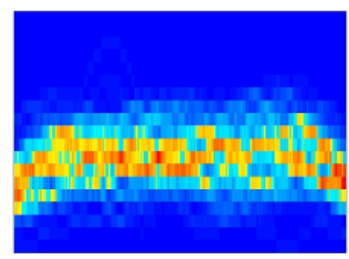

$B: \operatorname{lv}=6$

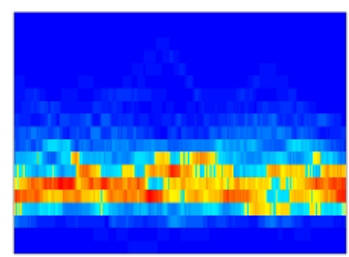

$F: I V=3$

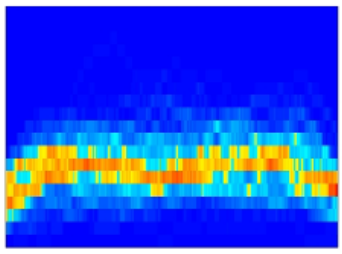

$F: I v=7$

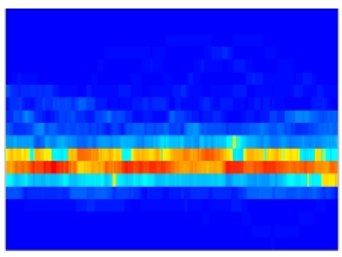

$B: I v=3$

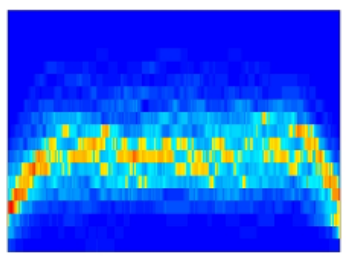

$B: l v=7$

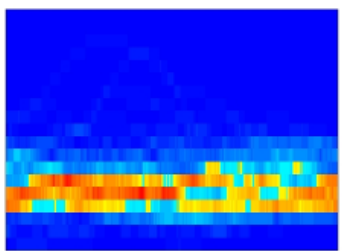

$F: I V=4$

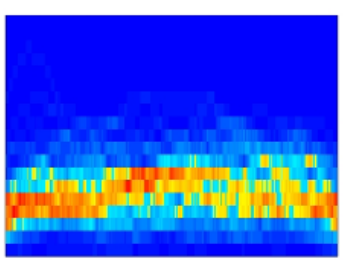

$F: I V=8$

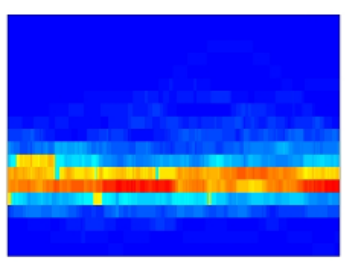

$B: I v=4$

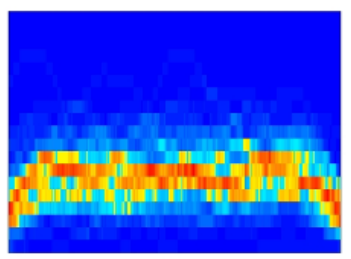

$B: l v=8$

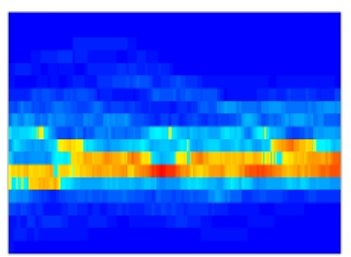

Figure A.3. Empirical Distribution for $F_{12, t}\left(\tau_{j}\right)$ and $B_{12, t}\left(\tau_{j}\right)$ using 100 replications of the process presented in Example 3.3 with $a=0.6$ and $b=0.3$. 
$F: I V=1$

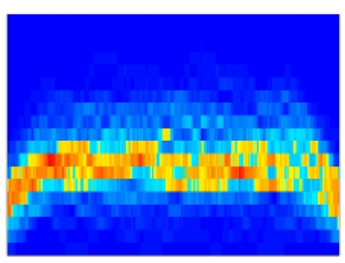

$F: I V=5$

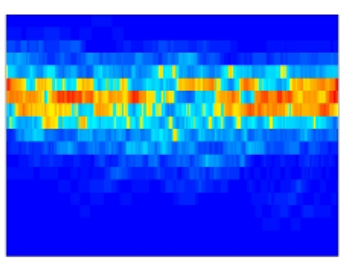

$B: I v=1$

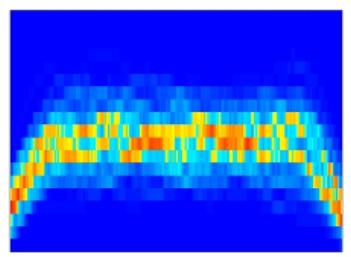

$B: I v=5$

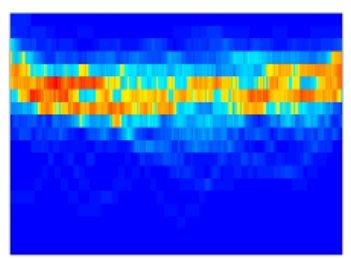

$F: I V=2$

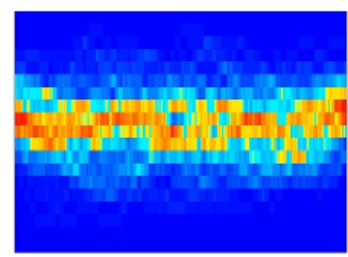

$F: I V=6$

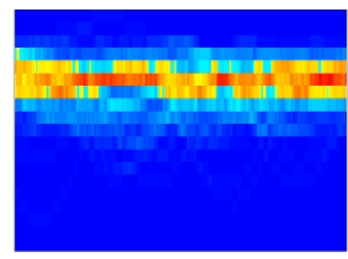

$B: I v=2$

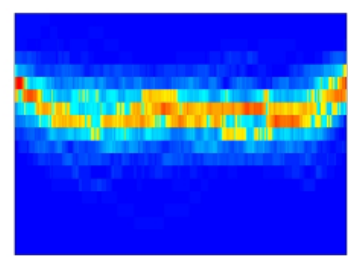

$B: I v=6$

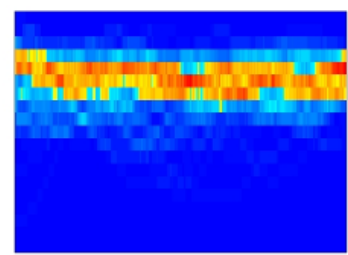

$F: I V=3$

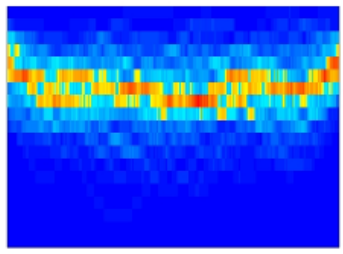

$F: I v=7$

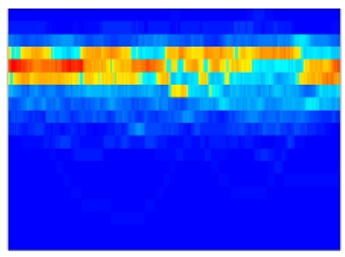

$B: I v=3$

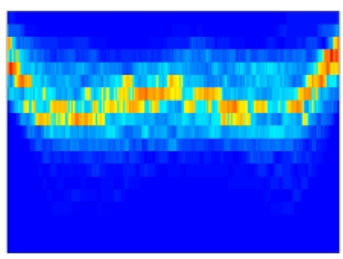

$B: I v=7$

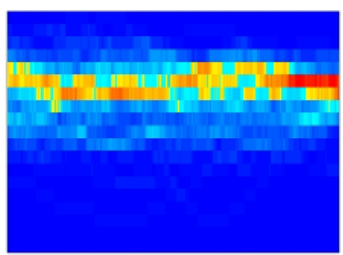

$F: I V=4$

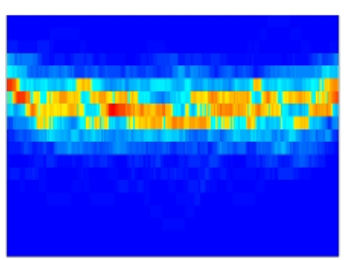

$F: I V=8$

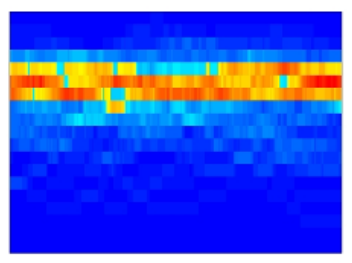

$B: l v=4$

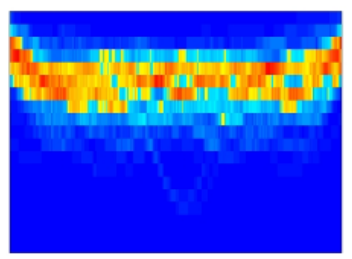

$B: I v=8$

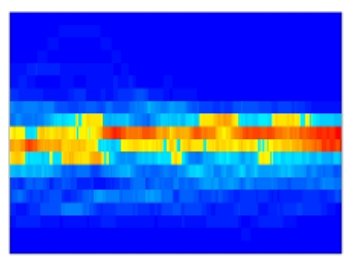

Figure A.4. Empirical Distribution for $F_{12, t}\left(\tau_{j}\right)$ and $B_{12, t}\left(\tau_{j}\right)$ using 100 replications of the process presented in Example 3.4. 

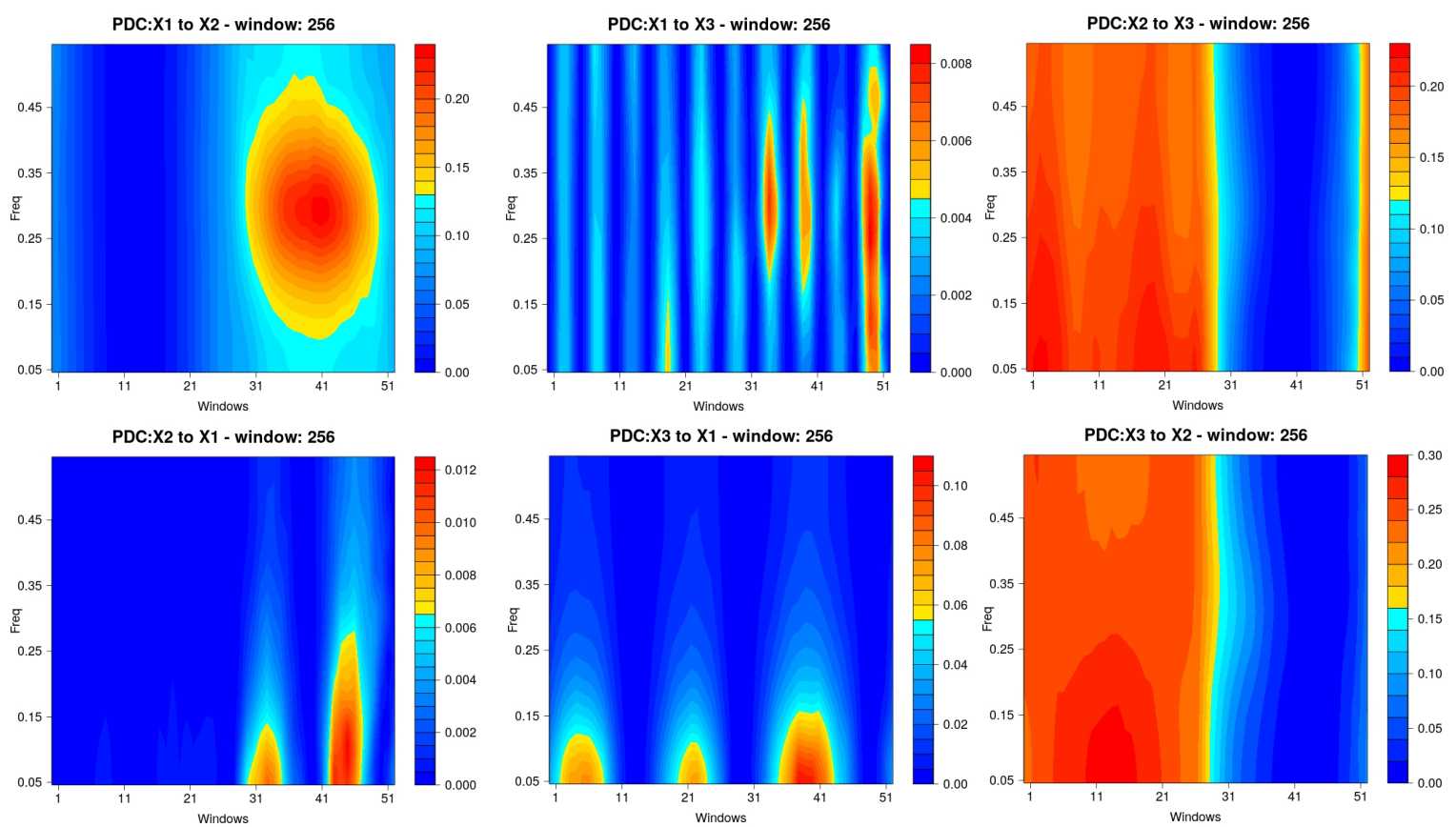

Figure A.5. Moving PDC using M=256 for Simulation 3 in Chapter 5.
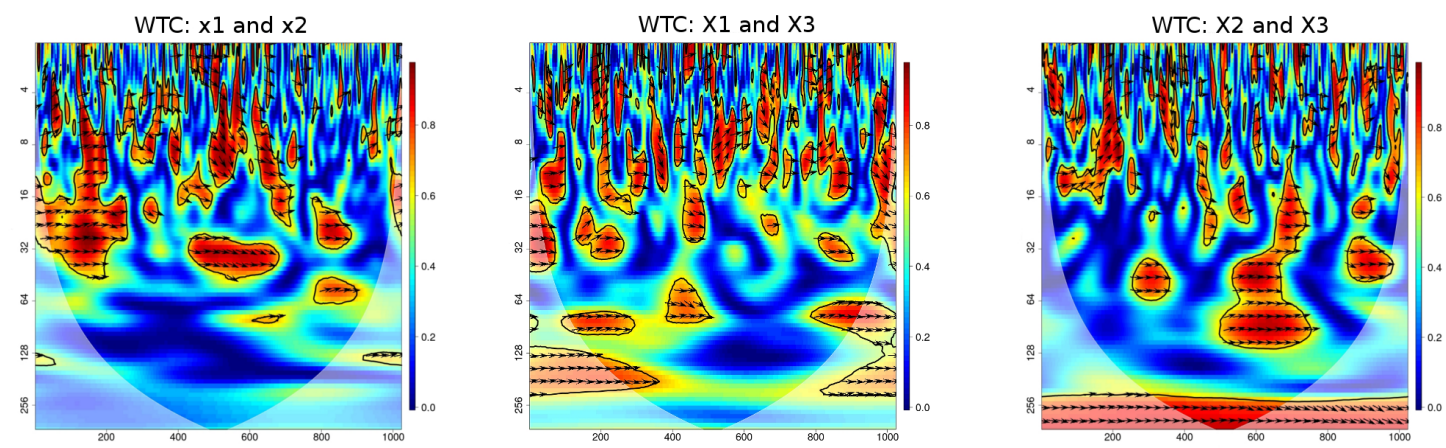

Figure A.6. Moving WTC for Simulation 3 in Chapter 5. 

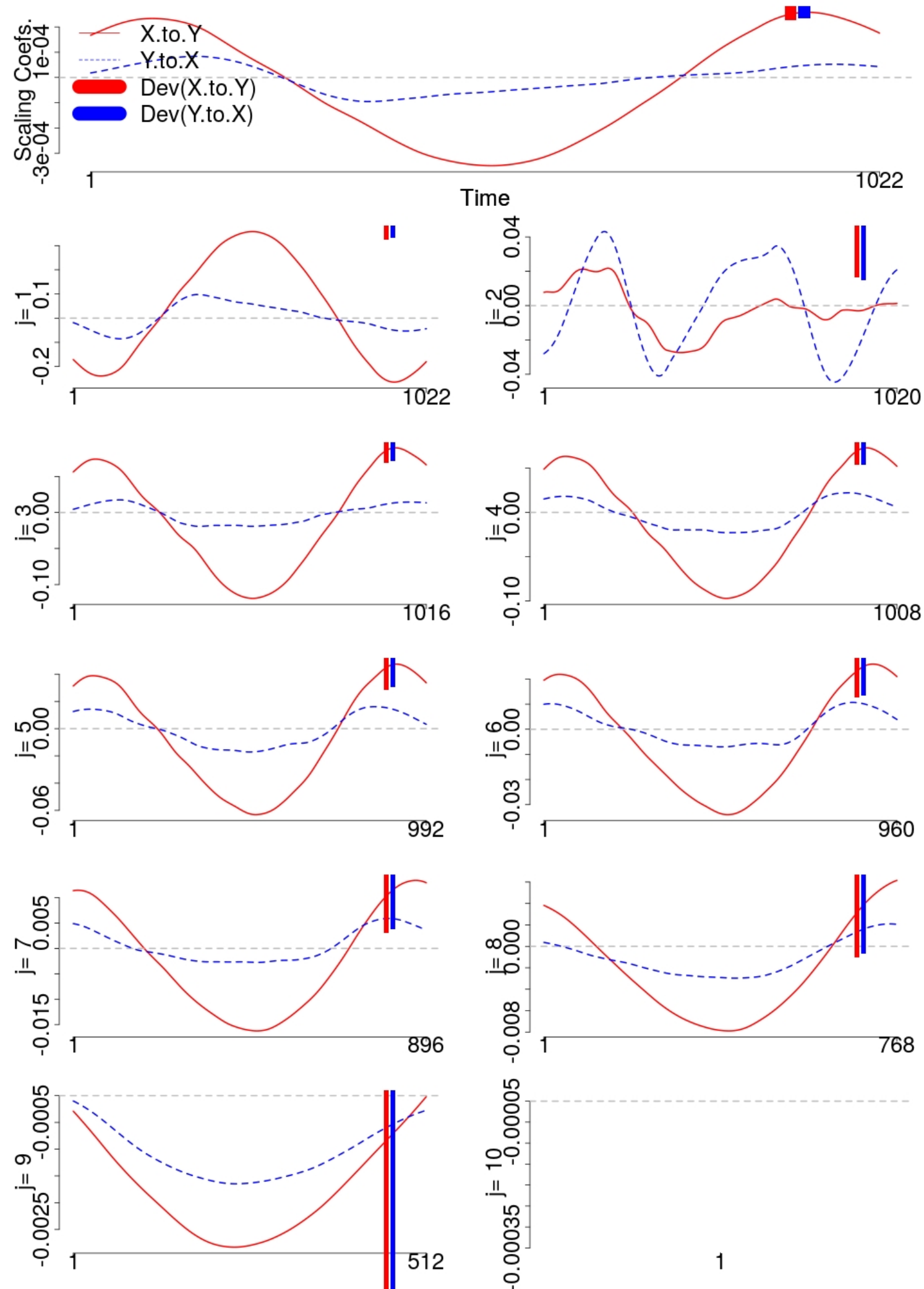

Figure A.7. $D W C$ between $X_{1, t}$ and $X_{2, t}$ for Simulation 3 in Chapter 5. 

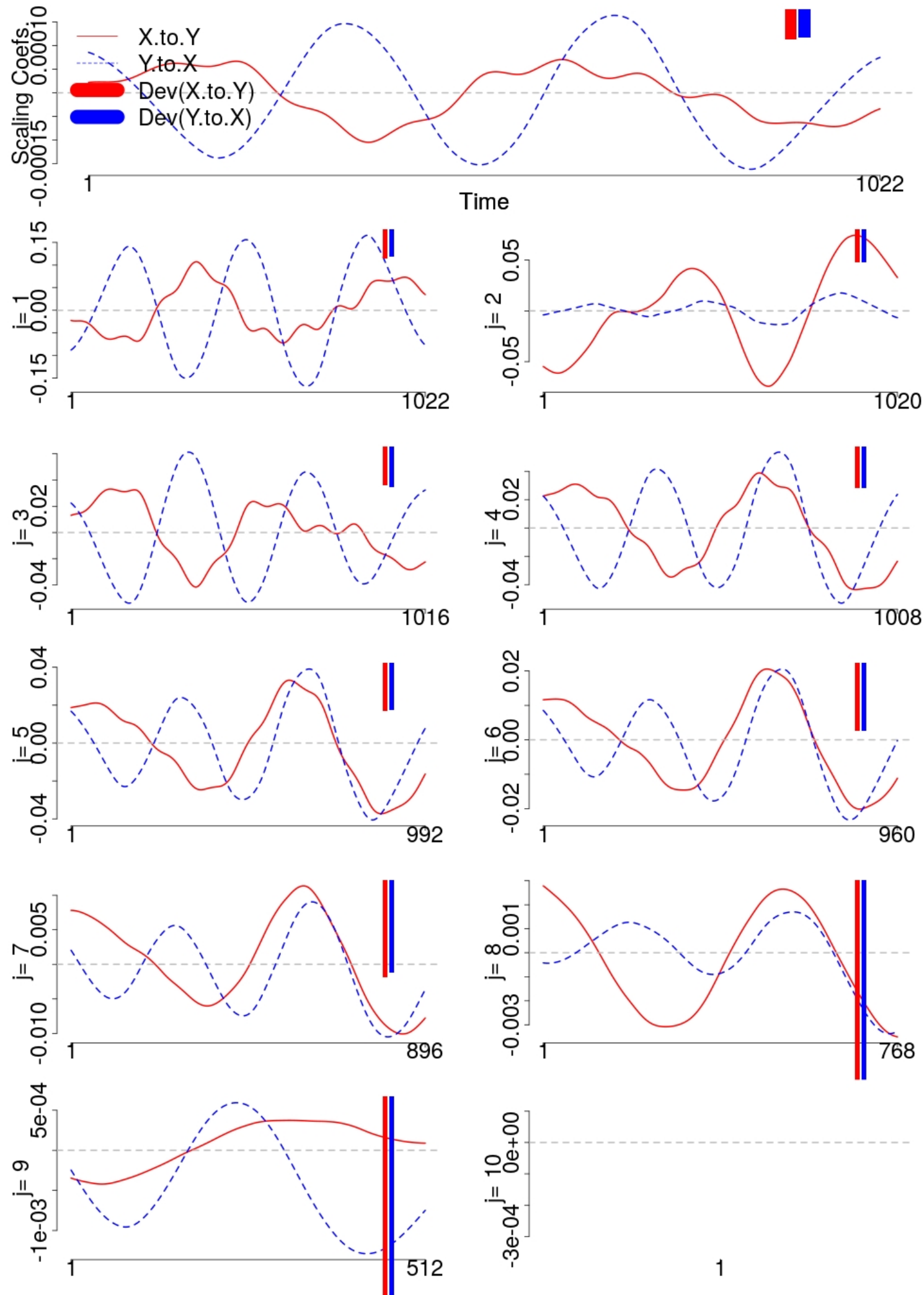

Figure A.8. $D W C$ between $X_{1, t}$ and $X_{3, t}$ for Simulation 3 in Chapter 5. 

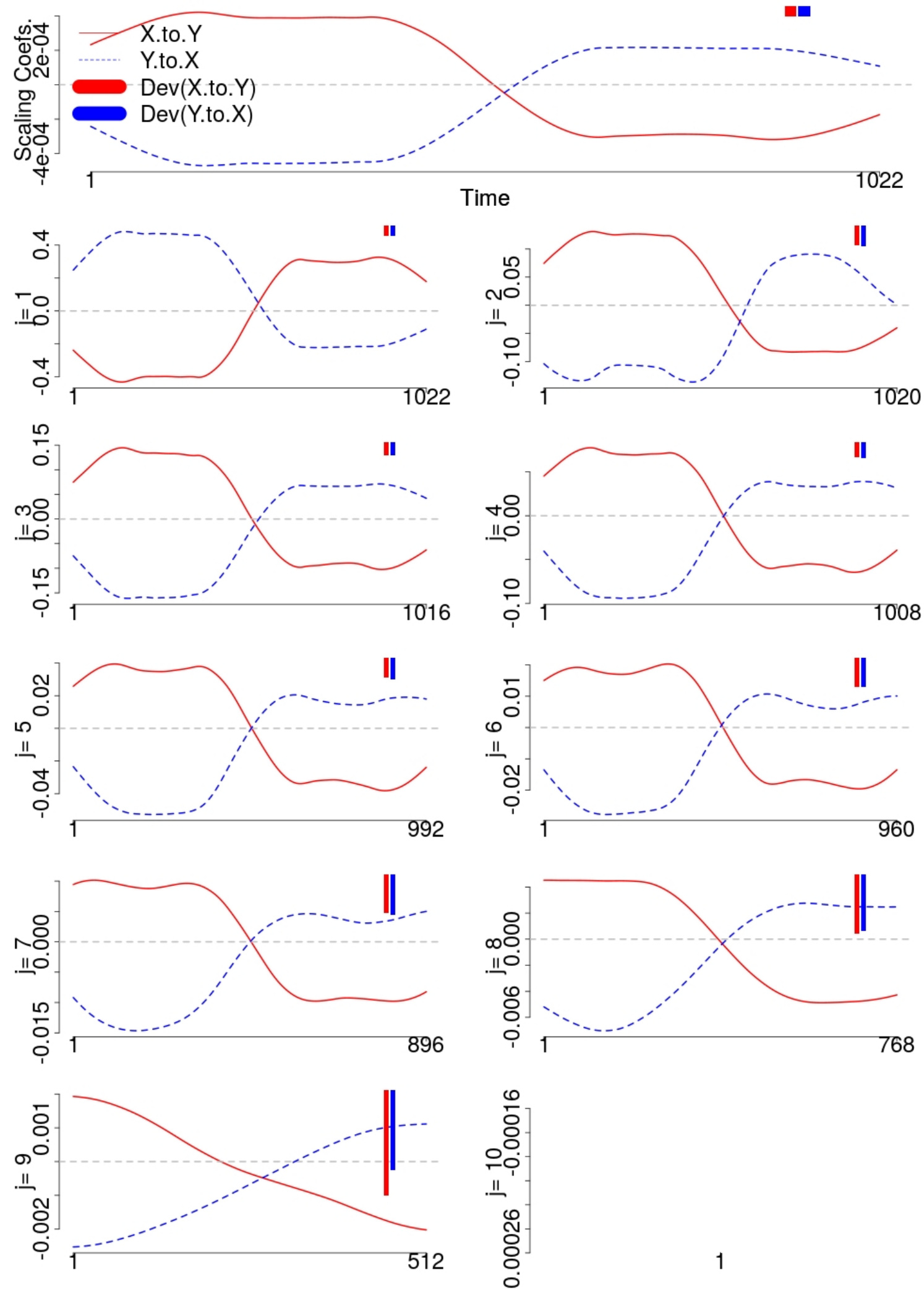

Figure A.9. $D W C$ between $X_{2, t}$ and $X_{3, t}$ for Simulation 3 in Chapter 5. 

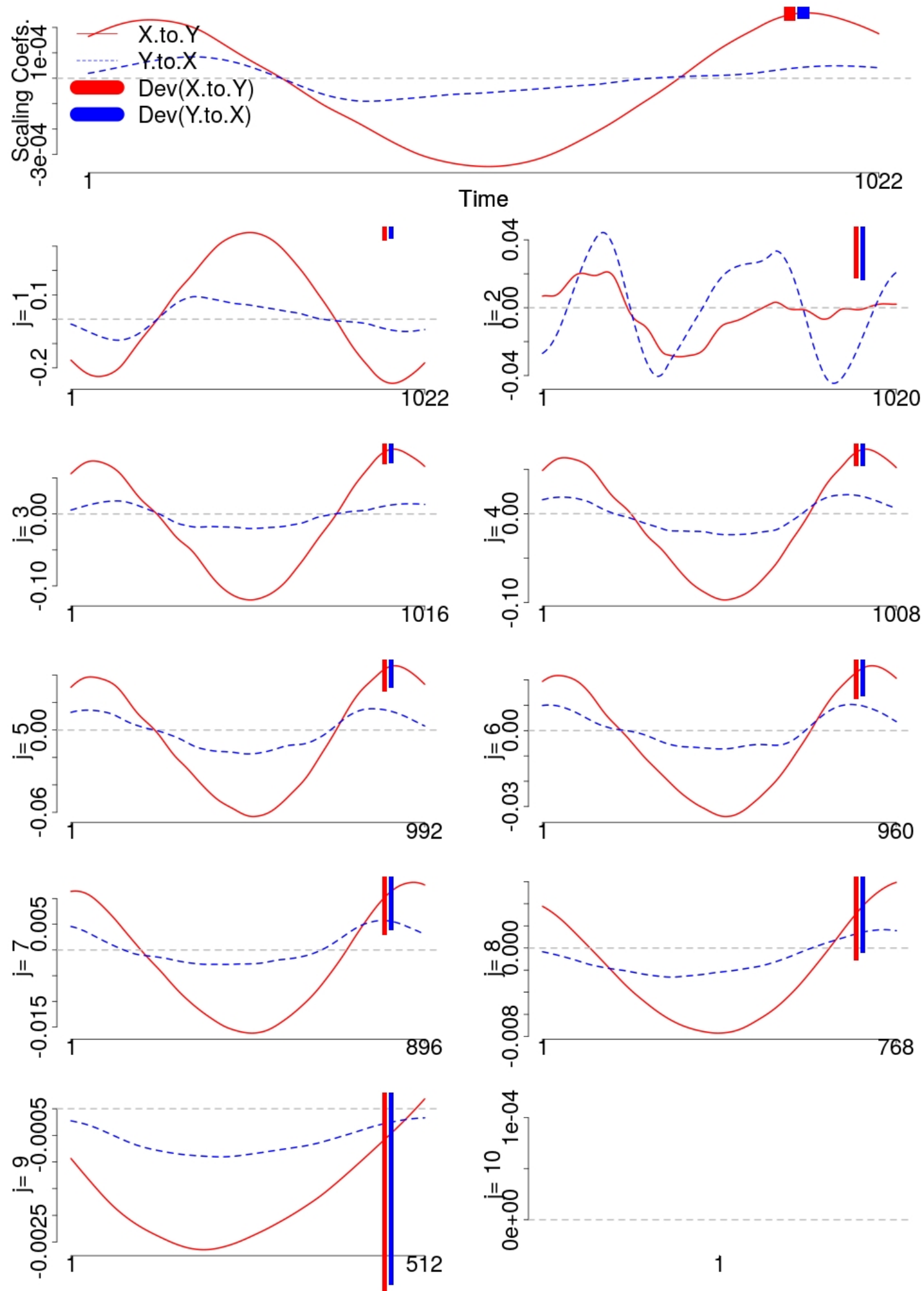

Figure A.10. $p D W C$ between $X_{1, t}$ and $X_{2, t}$ for Simulation 3 in Chapter 5. 

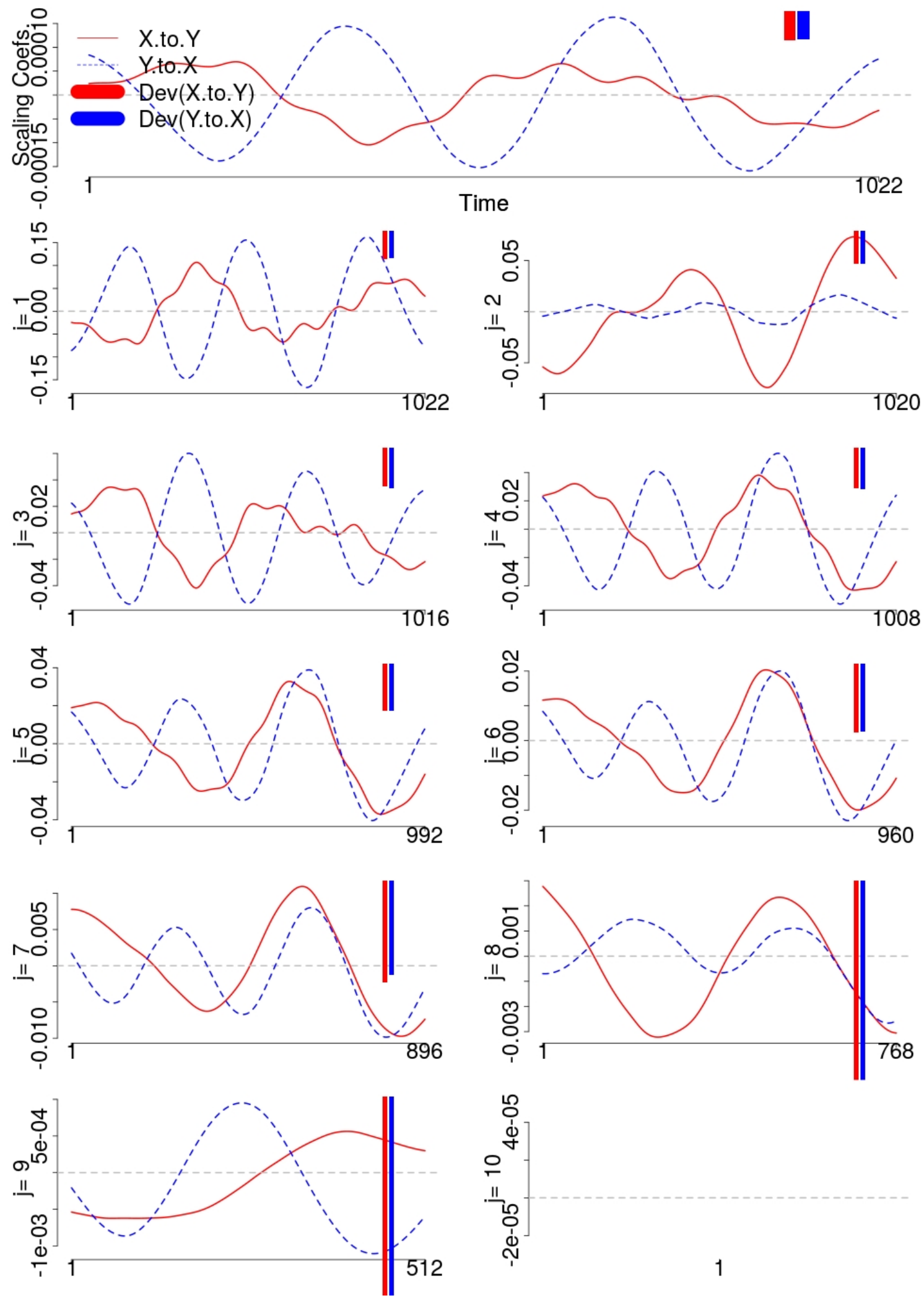

Figure A.11. $p D W C$ between $X_{1, t}$ and $X_{3, t}$ for Simulation 3 in Chapter 5. 

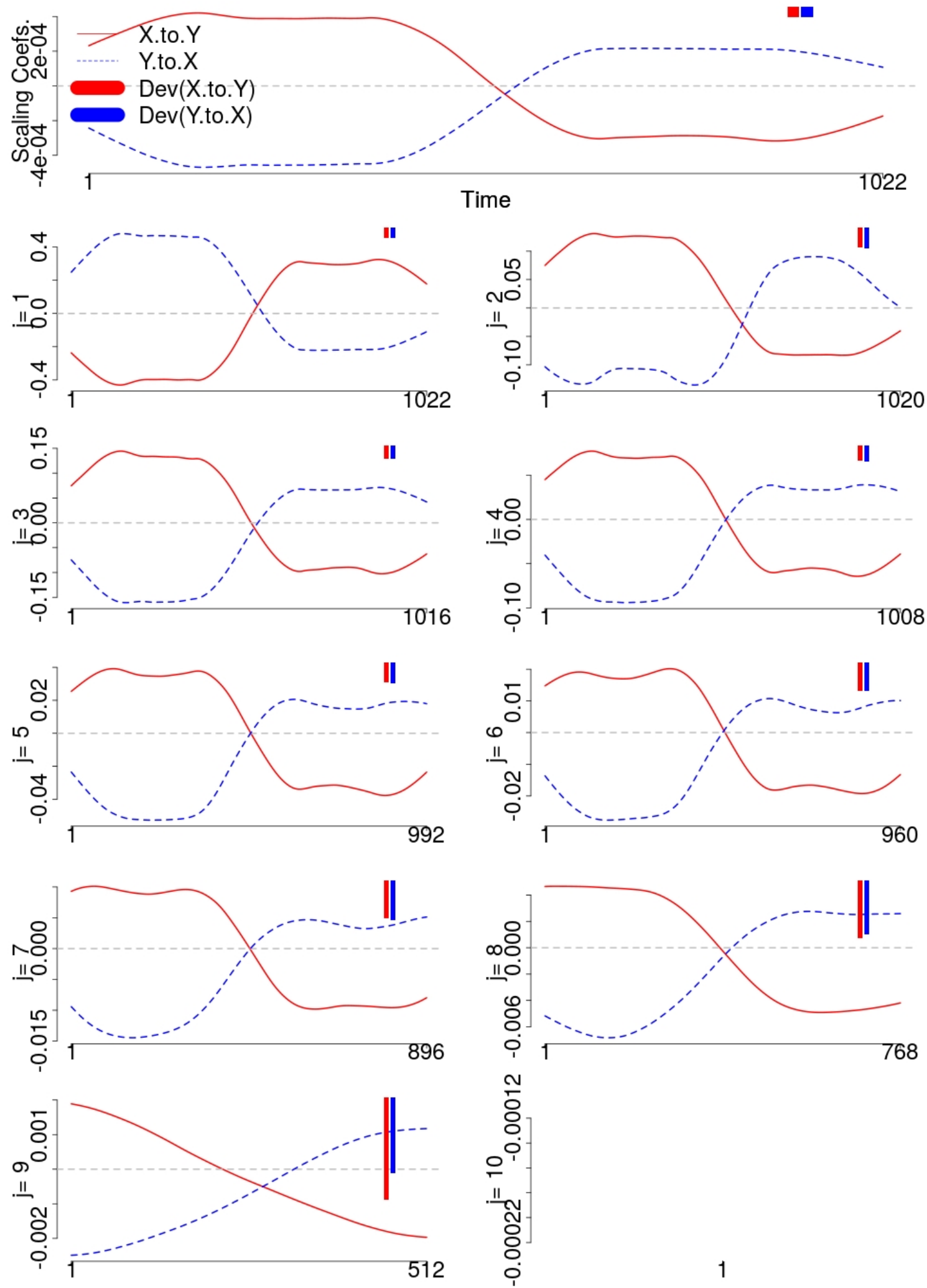

Figure A.12. $p D W C$ between $X_{2, t}$ and $X_{3, t}$ for Simulation 3 in Chapter 5. 

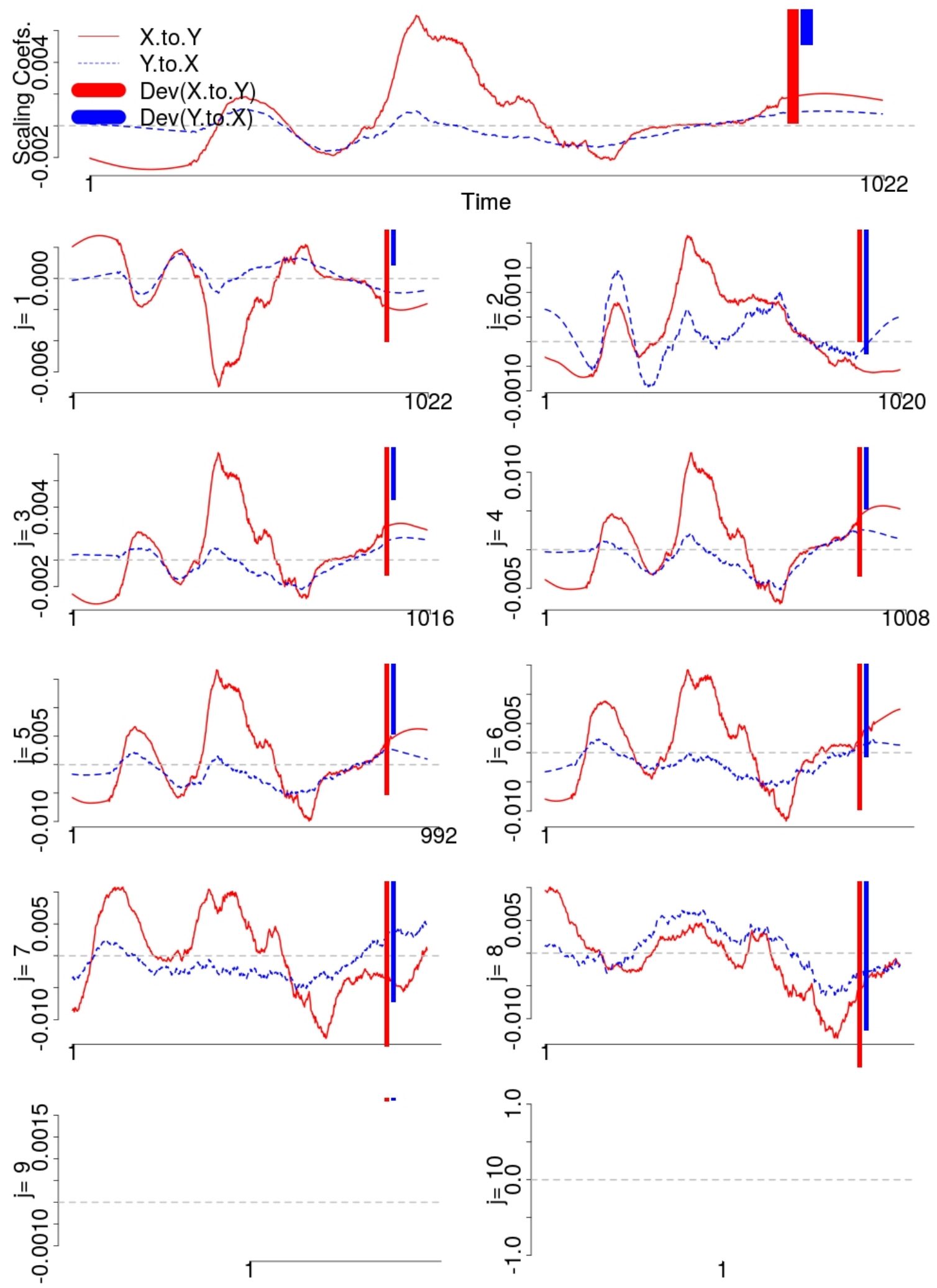

Figure A.13. ppDWC between $X_{1, t}$ and $X_{2, t}$ for Simulation 3 in Chapter 5 . 

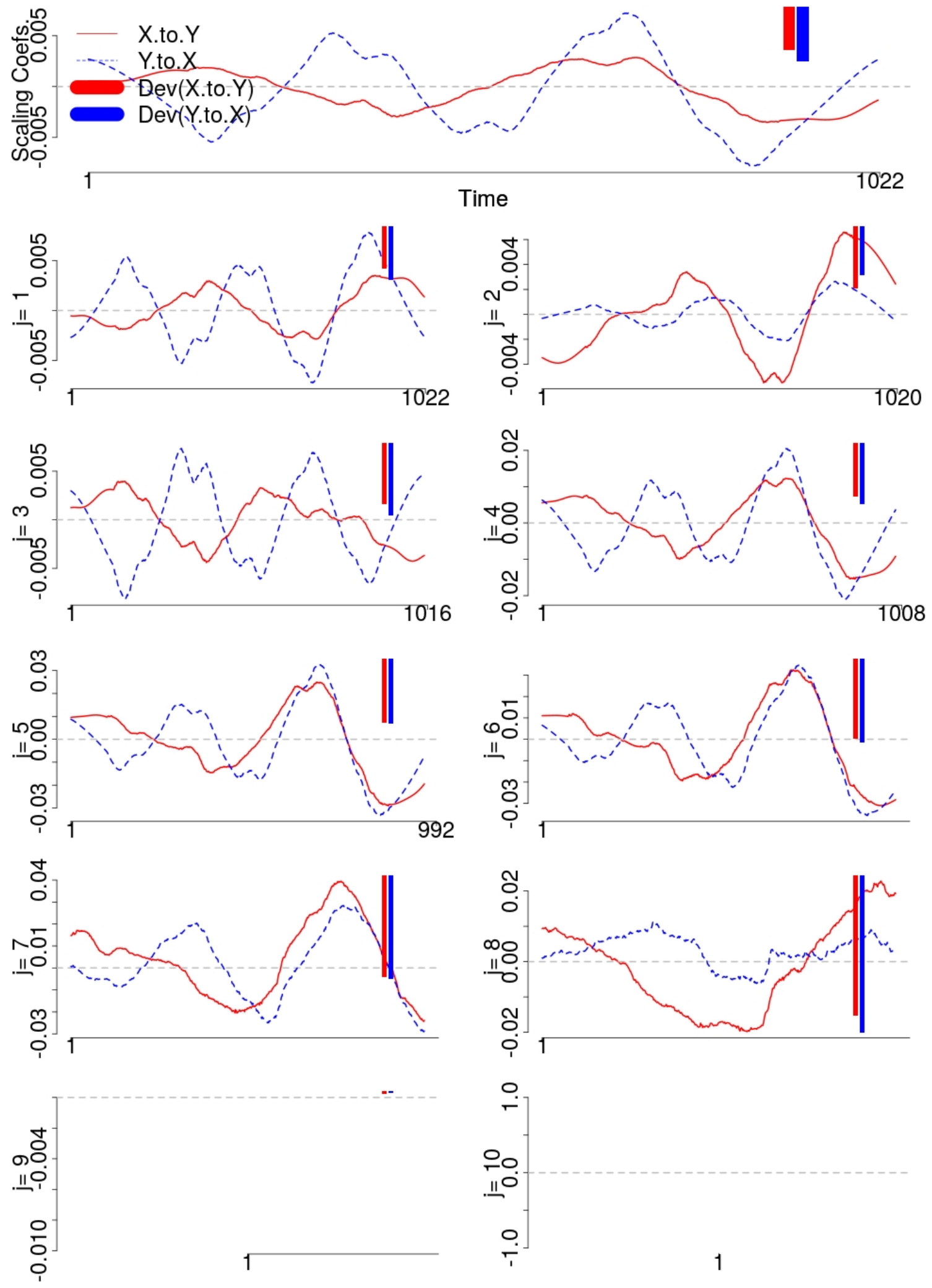

Figure A.14. $p p D W C$ between $X_{1, t}$ and $X_{3, t}$ for Simulation 3 in Chapter 5. 

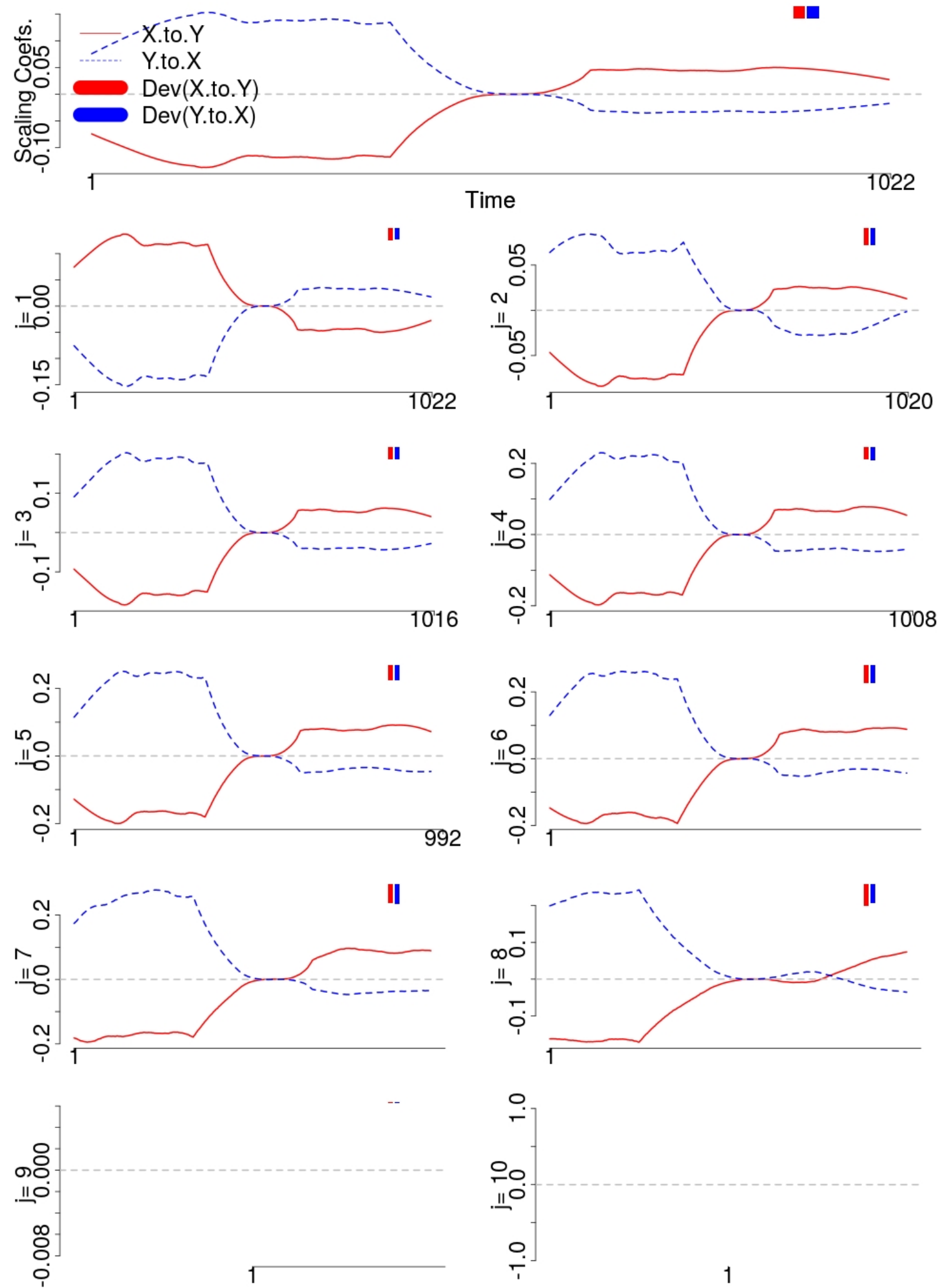

Figure A.15. $p p D W C$ between $X_{2, t}$ and $X_{3, t}$ for Simulation 3 in Chapter 5. 

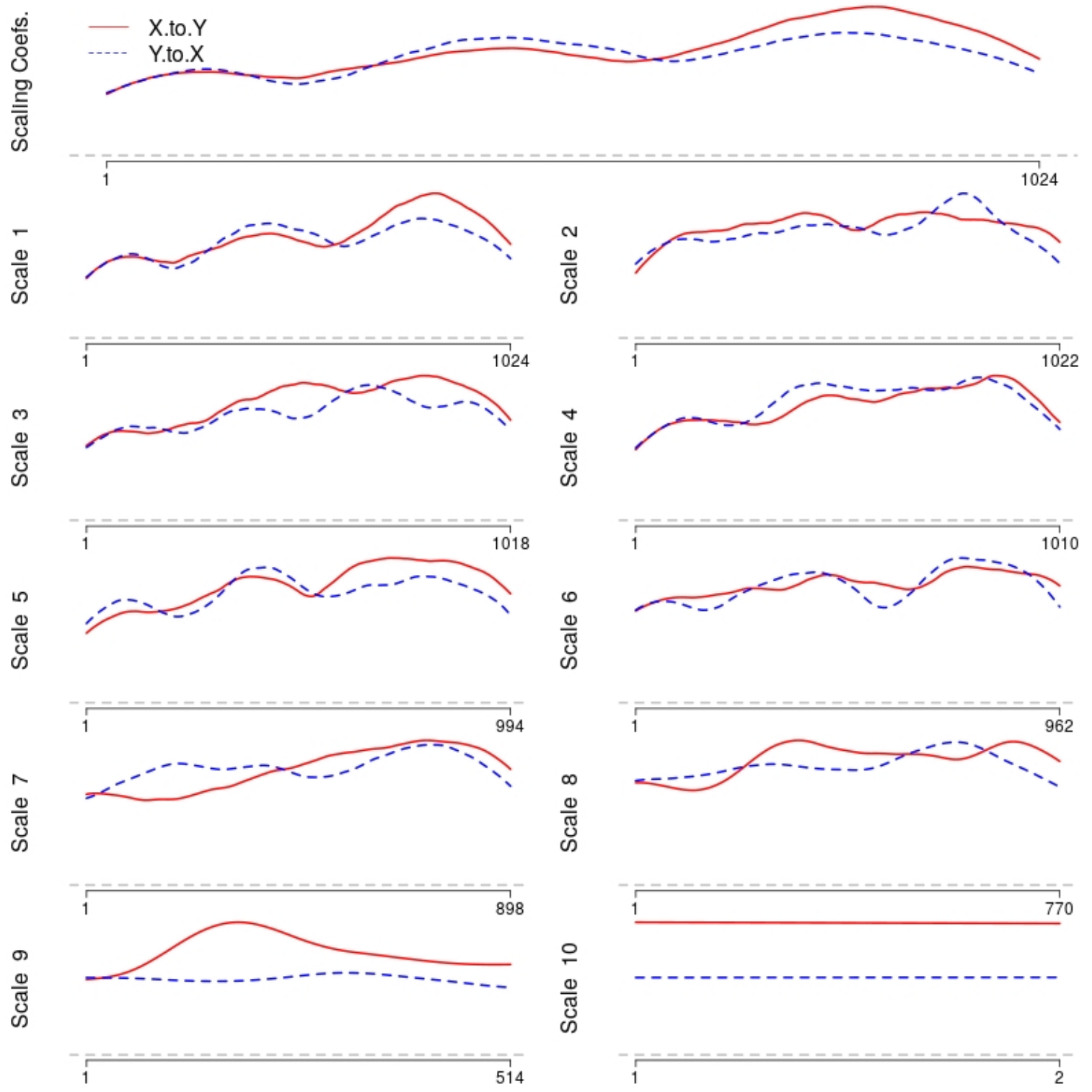

Figure A.16. Standard Deviations for DWC in Simulation 1. 

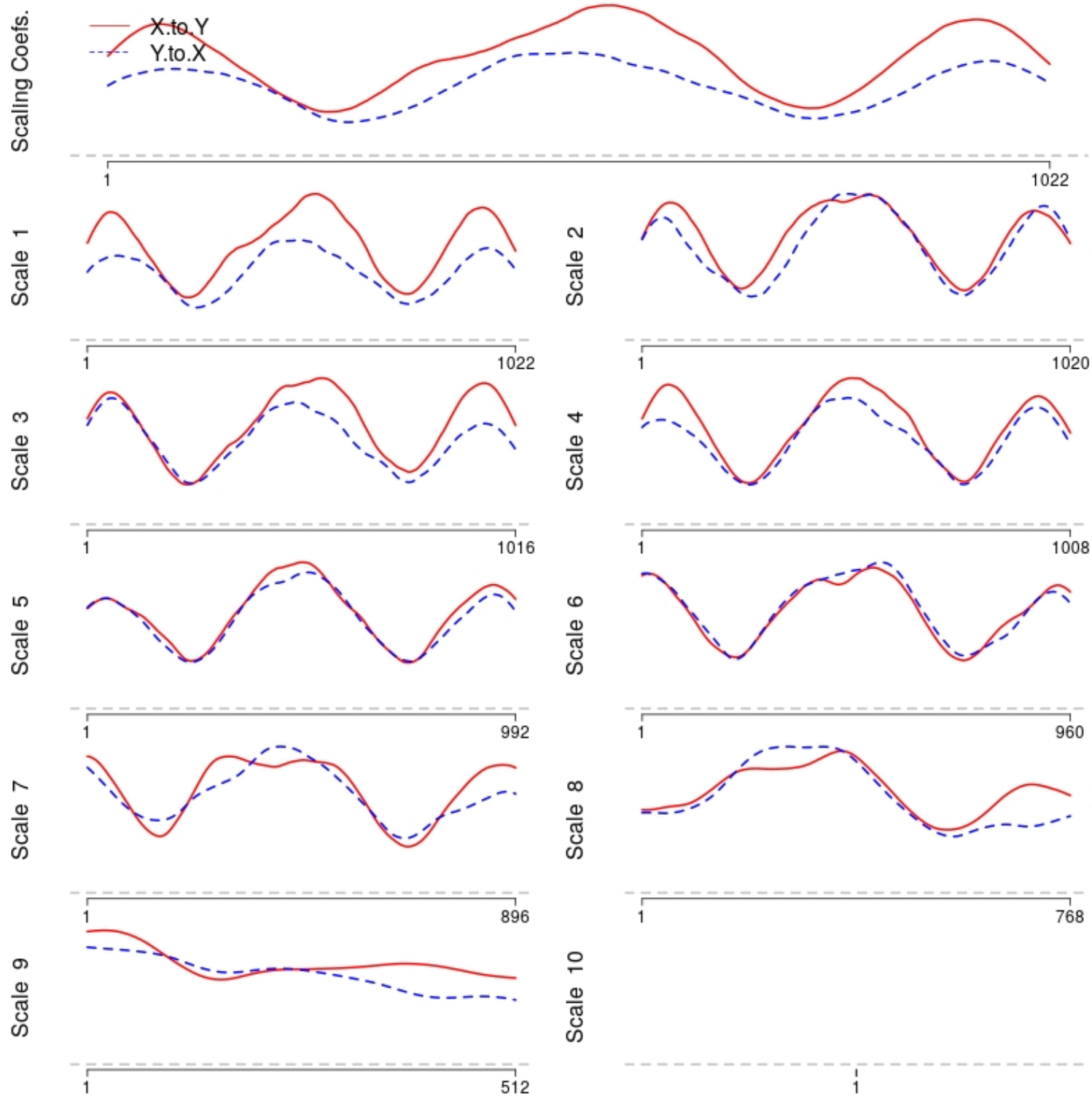

Figure A.17. Standard Deviations for DWC in Simulation 2. 

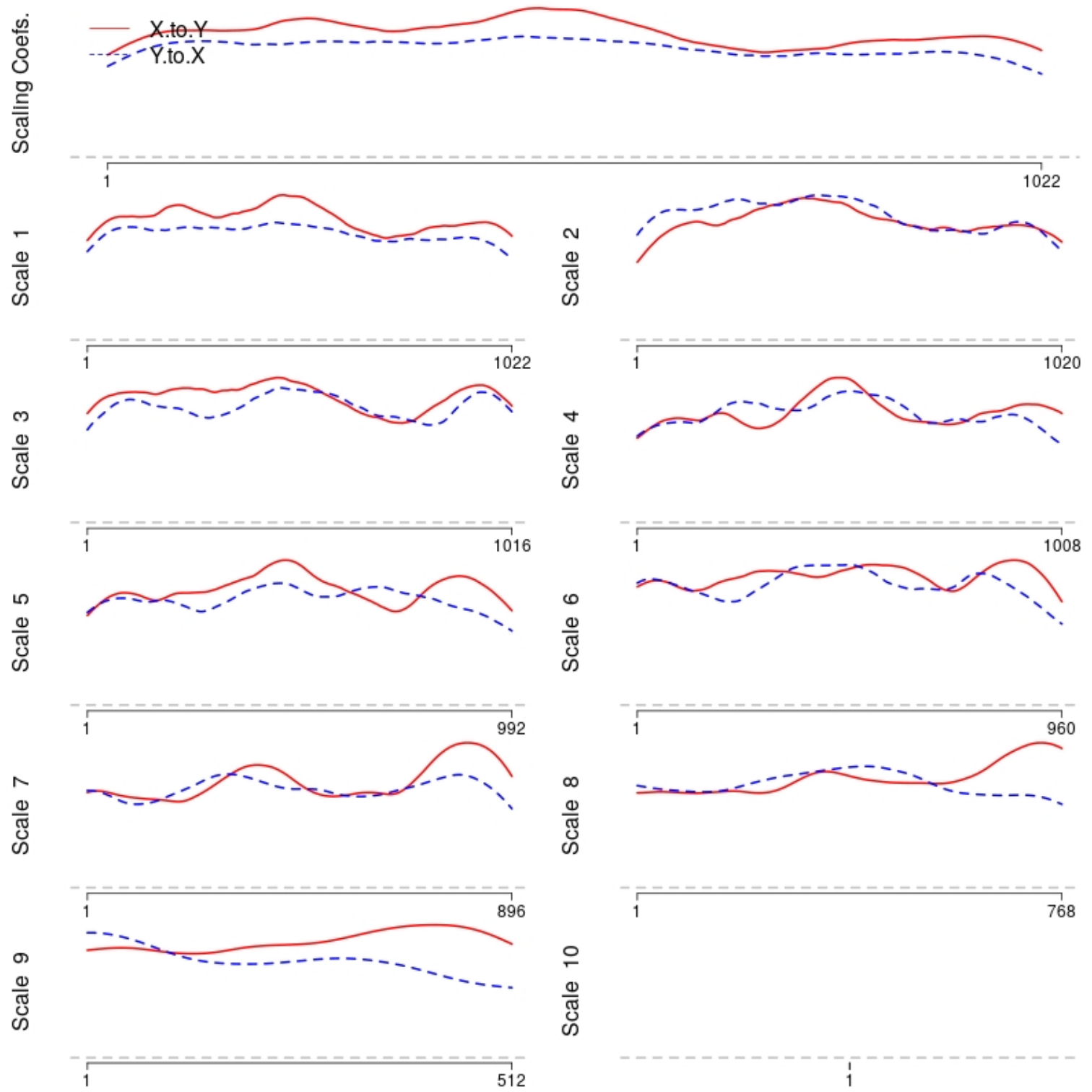

Figure A.18. Standard Deviations for DWC in Simulation 3: $X_{1, t}$ and $X_{2, t}$. 

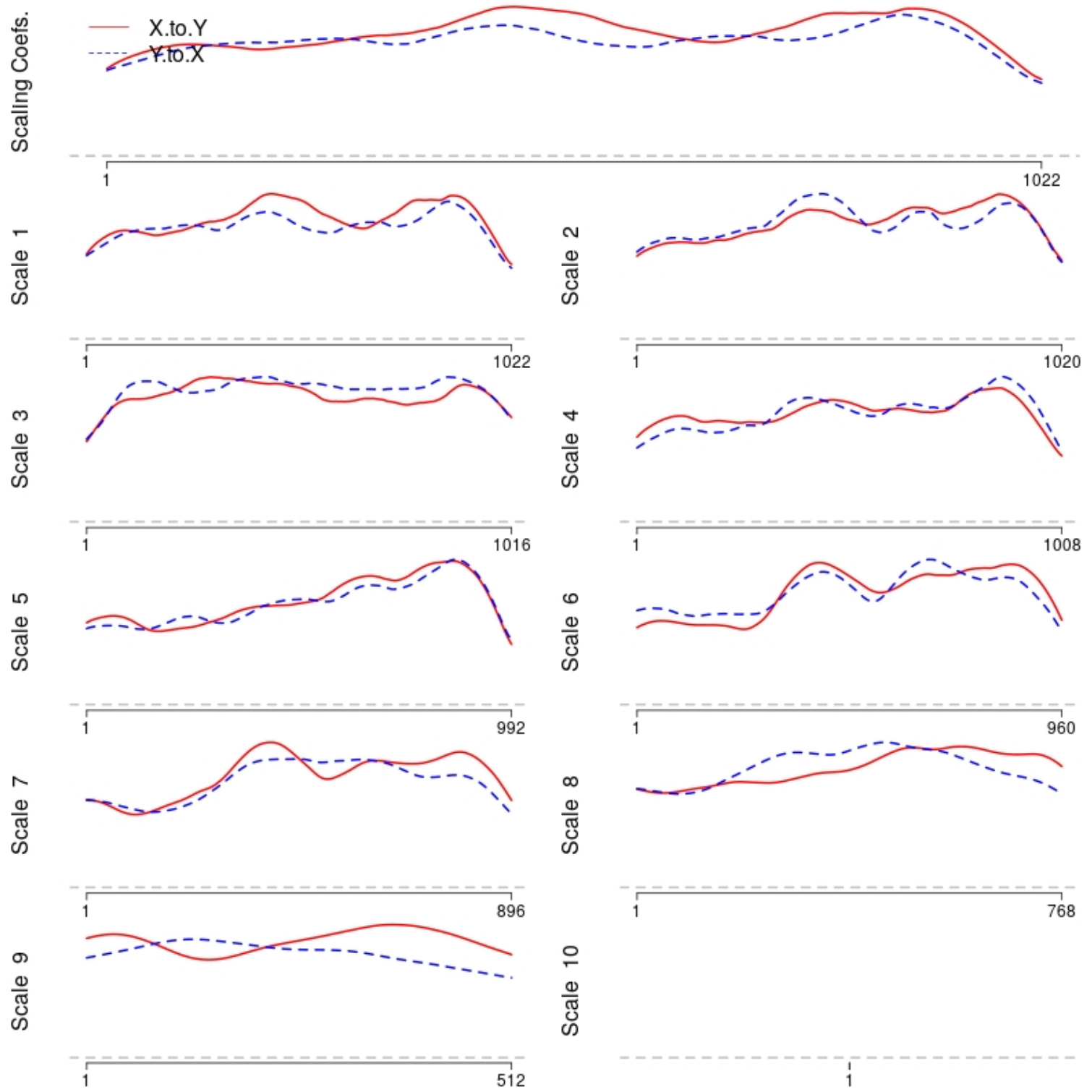

Figure A.19. Standard Deviations for DWC in Simulation 3: $X_{1, t}$ and $X_{3, t}$. 

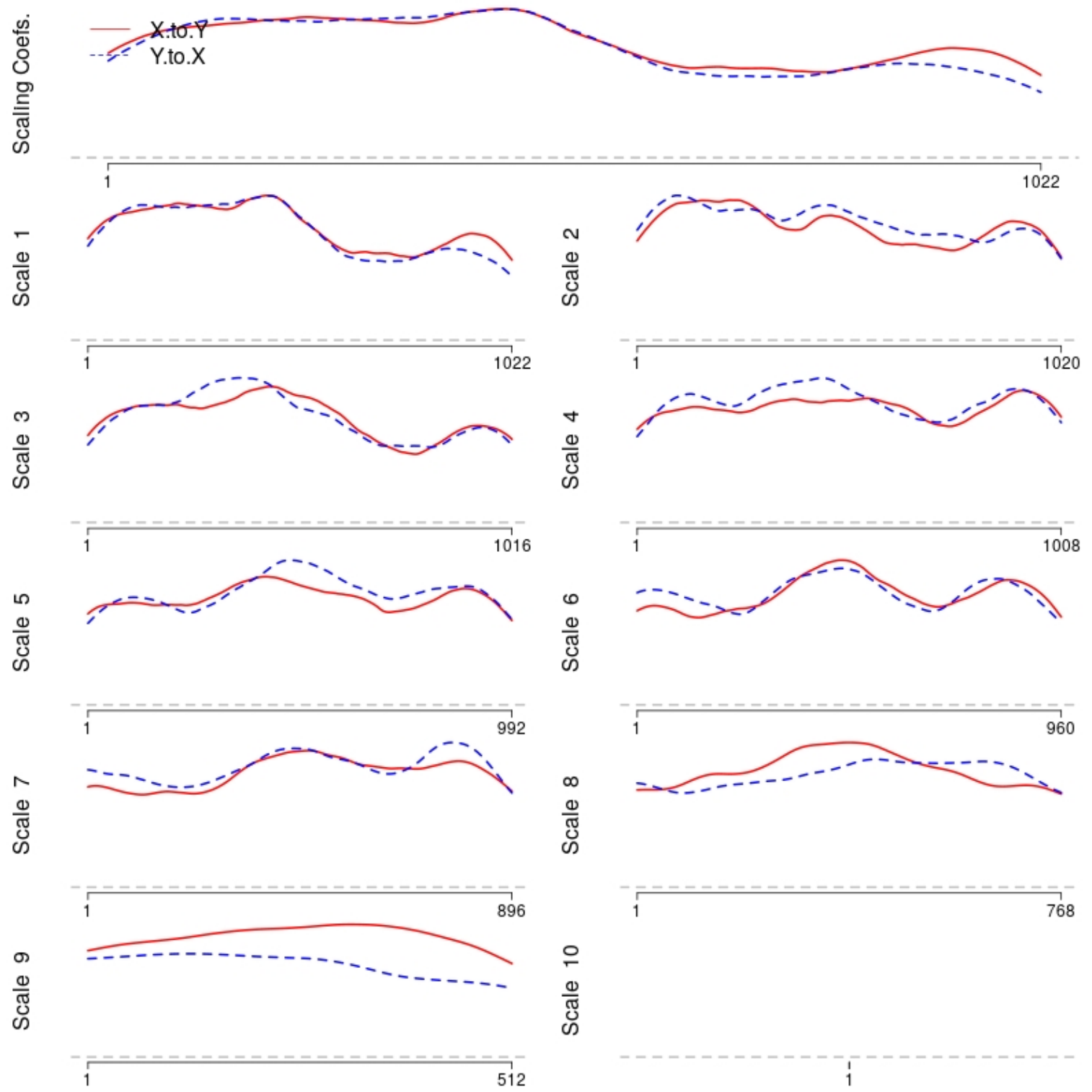

Figure A.20. Standard Deviations for DWC in Simulation 3: $X_{2, t}$ and $X_{3, t}$. 

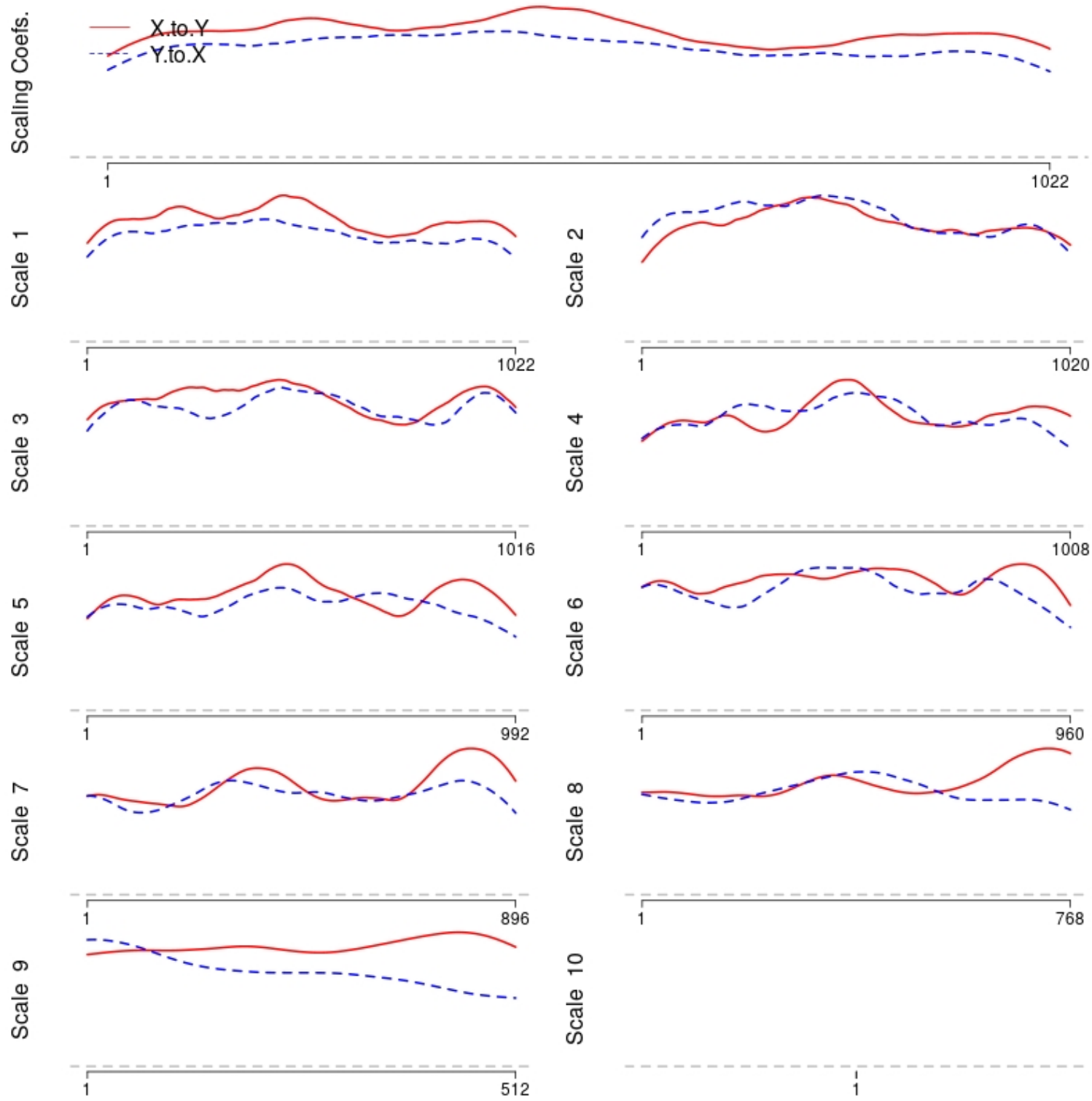

Figure A.21. Standard Deviations for $p D W C$ in Simulation 3: $X_{1, t}$ and $X_{2, t}$. 

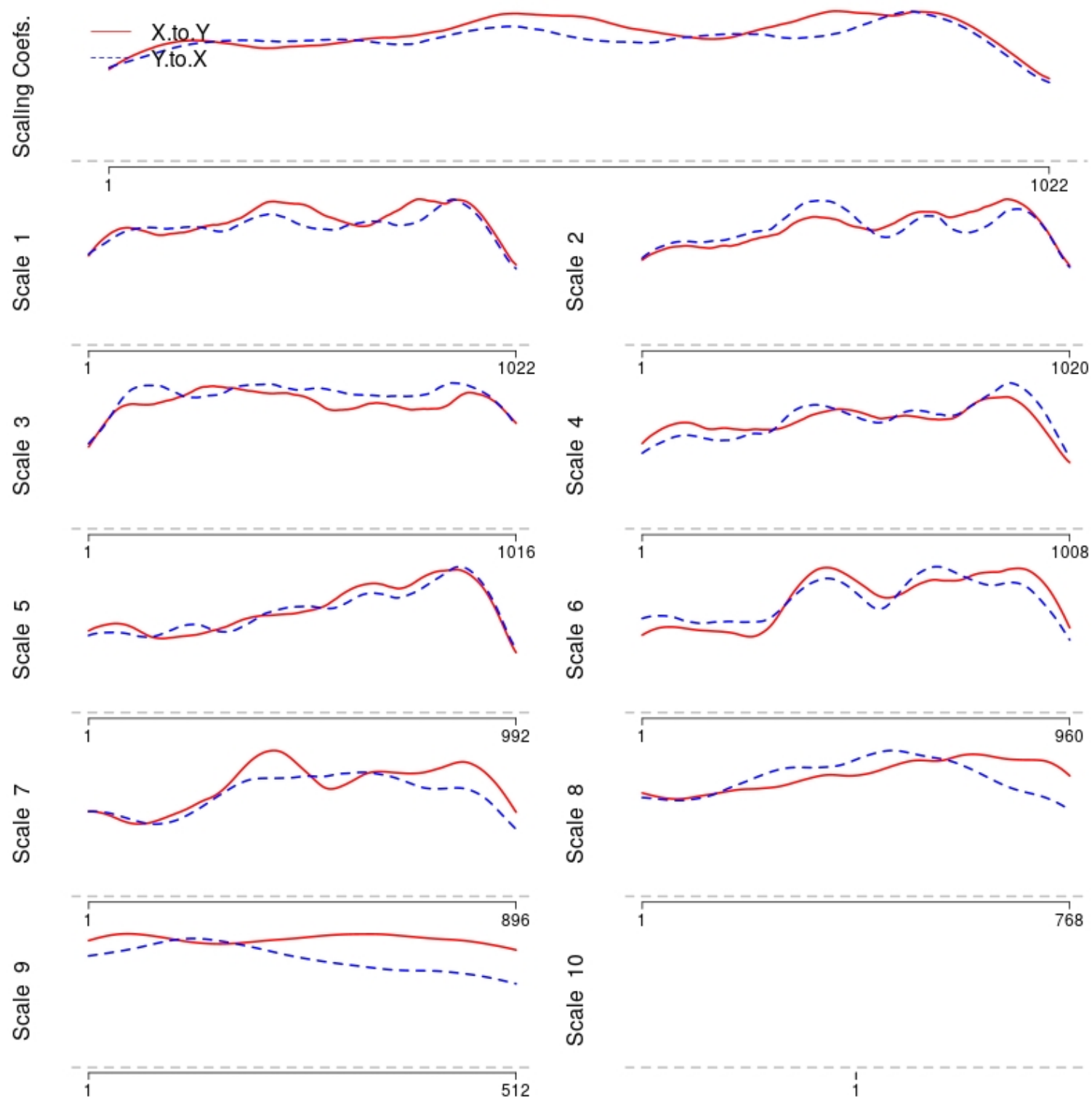

Figure A.22. Standard Deviations for $p D W C$ in Simulation 3: $X_{1, t}$ and $X_{3, t}$. 

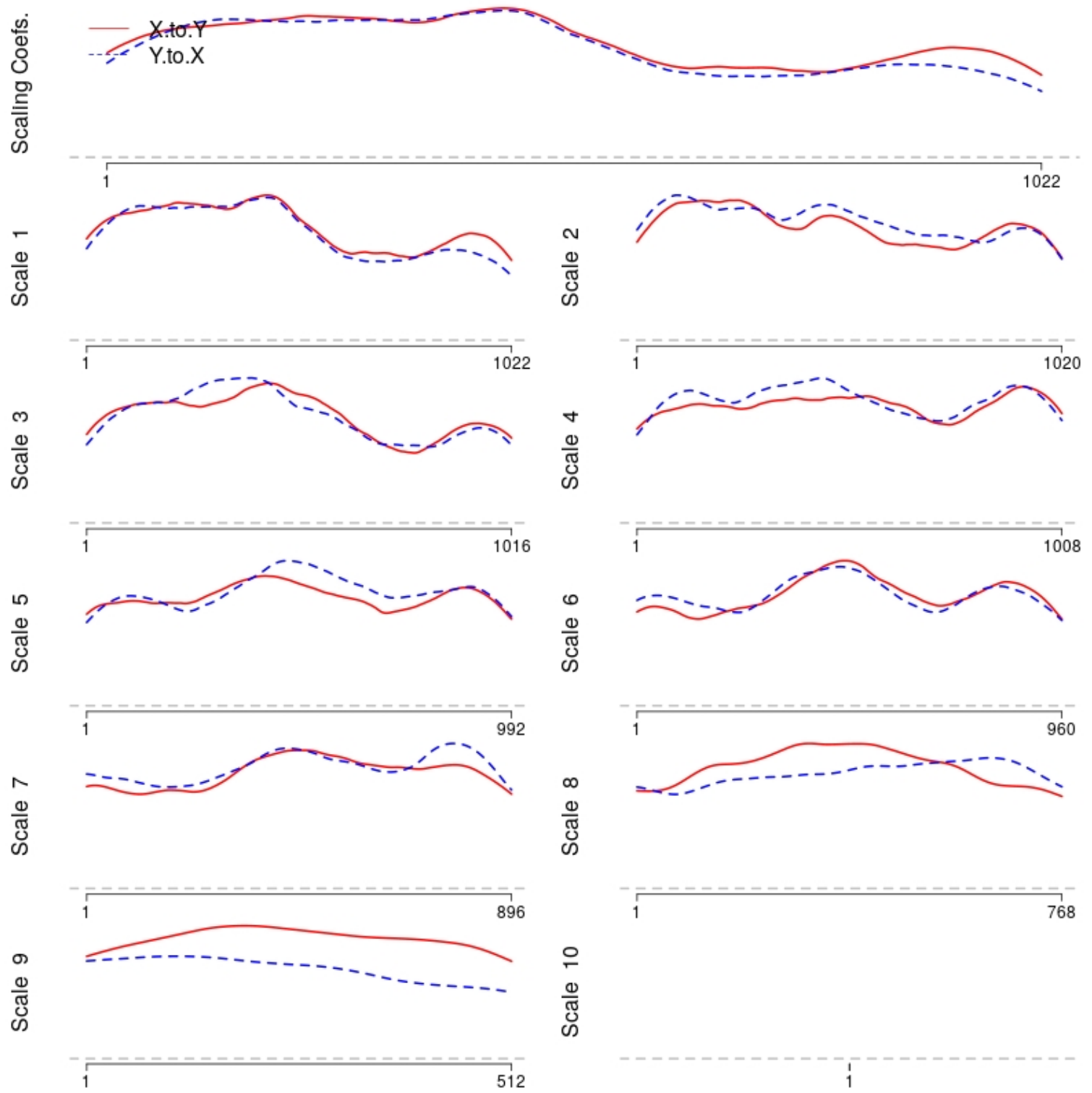

Figure A.23. Standard Deviations for $p D W C$ in Simulation 3: $X_{2, t}$ and $X_{3, t}$. 

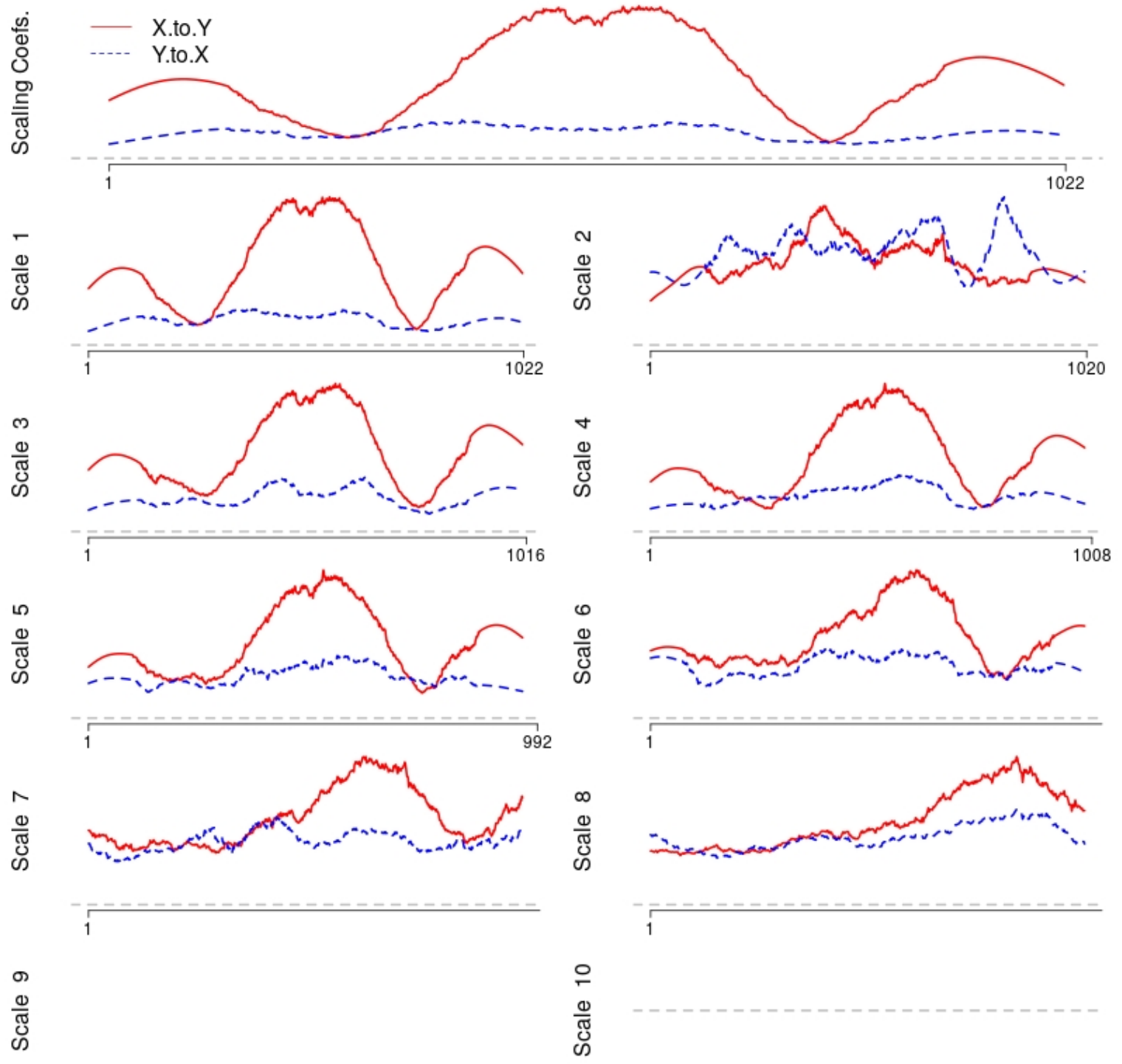

Figure A.24. Standard Deviations for ppDWC in Simulation 3: $X_{1, t}$ and $X_{2, t}$. 

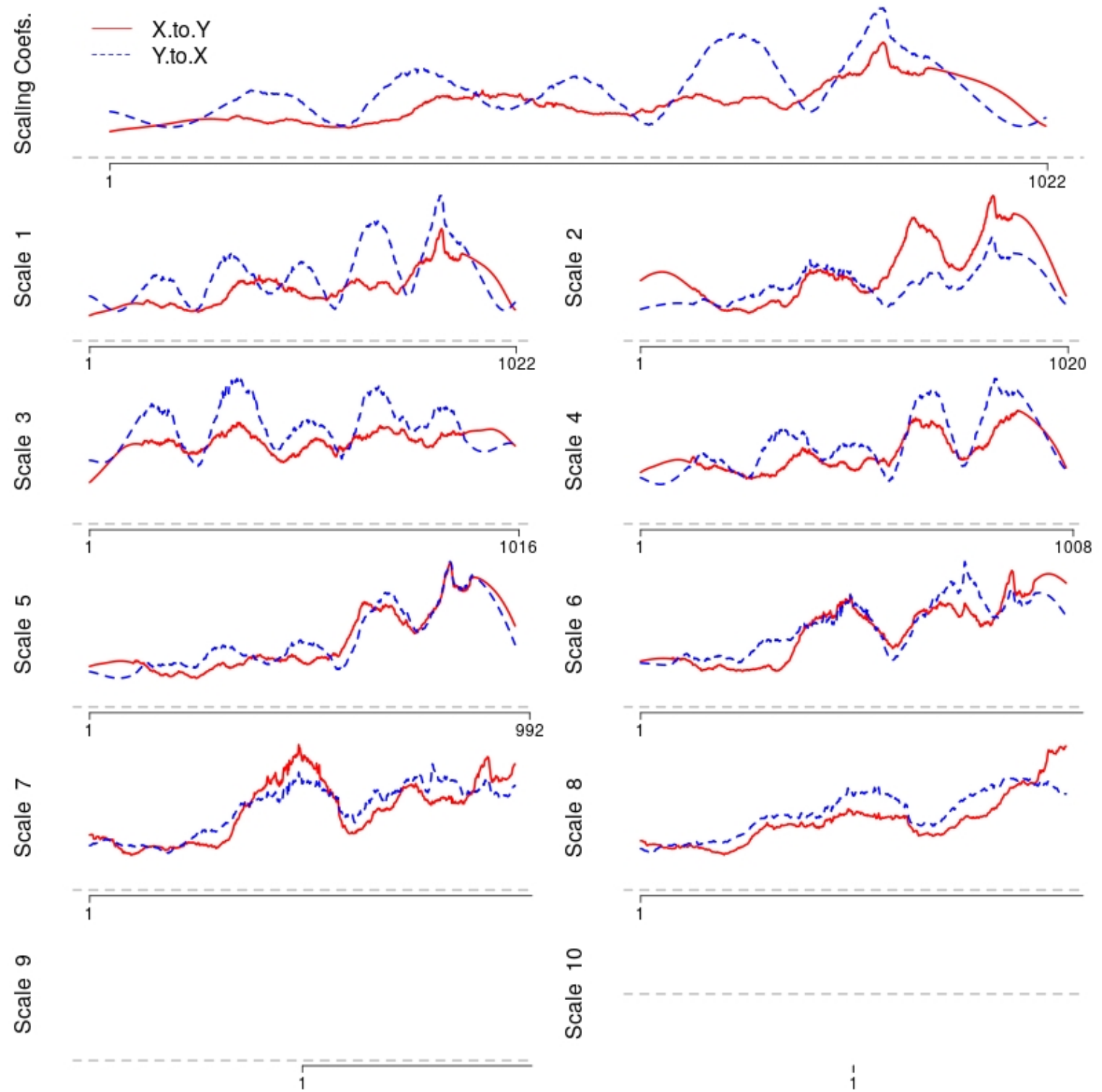

Figure A.25. Standard Deviations for ppDWC in Simulation 3: $X_{1, t}$ and $X_{3, t}$. 


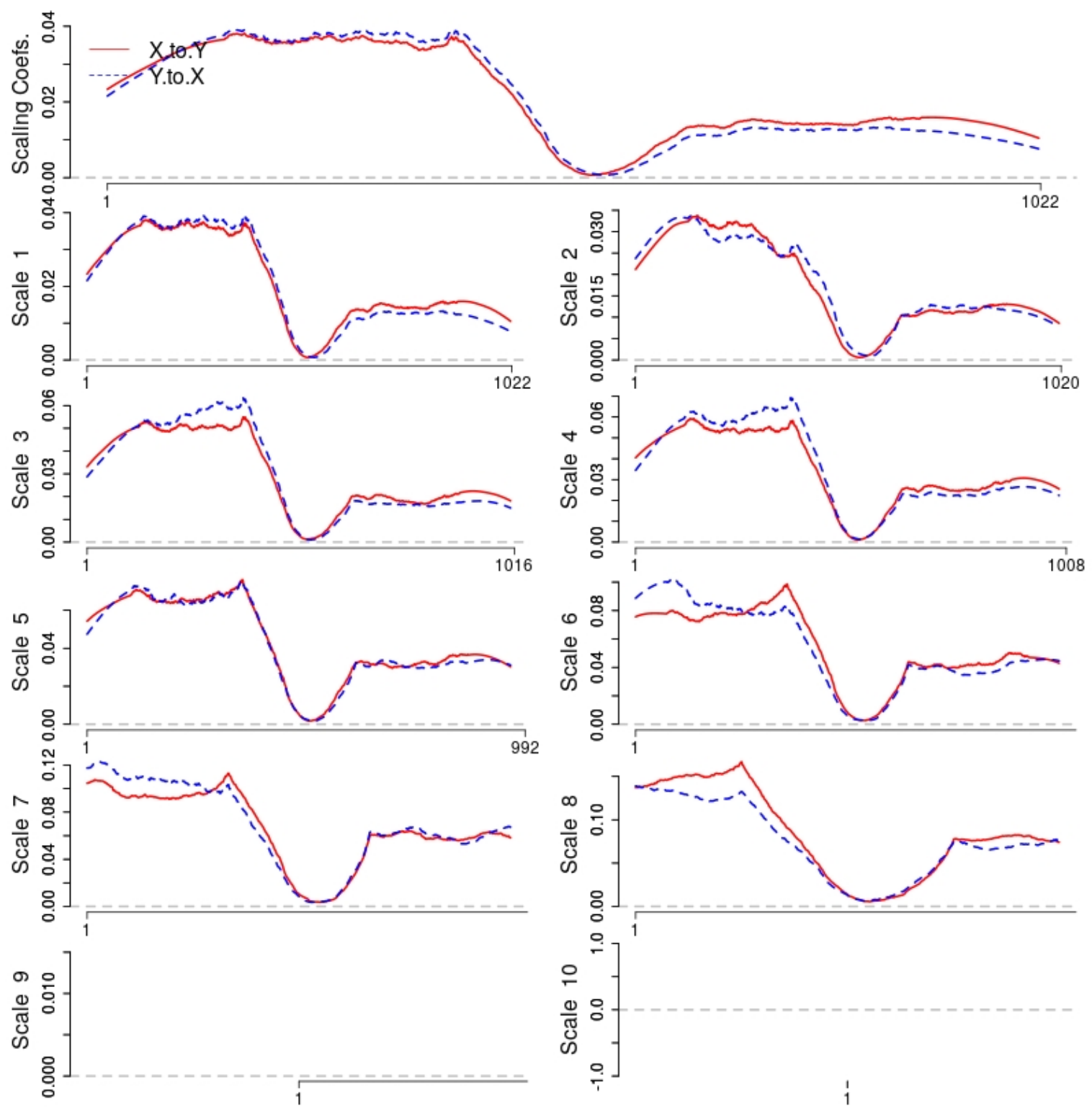

Figure A.26. Standard Deviations for ppDWC in Simulation 3: $X_{2, t}$ and $X_{3, t}$. 


\section{Appendix B}

\section{Proofs}

Proof of Proposition 3.1. Consider the wavelet covariance defined in (2.45). Notice that circularity assumption causes inversions in temporal-causal relations between $X_{t}$ and $Y_{t}$ that must be avoided. If we consider only $t \in \Upsilon=\left\{t \mid t \geq L_{j}\right.$ and $\left.t \leq T-L_{j}, \forall j\right\}$, i.e. values of $t$ that do not include the circularity assumption, then we can also eliminate the modulo operator from (2.44). Finally, with $\operatorname{Cov}\left(X_{t_{1}}, Y_{t_{2}}\right)=\gamma_{X Y}\left(t_{1}, t_{2}\right)=\gamma\left(t_{1}, t_{2}\right)$, we have for one particular value $j$ and an arbitrary value $t:$

$$
\nu_{X Y, t}\left(\tau_{j}\right)=\operatorname{Cov}\left(\widetilde{W}_{j, t}^{X}, \widetilde{W}_{j, t}^{Y}\right)=\mathbb{E}\left[\widetilde{W}_{j, t}^{X}\left(\widetilde{W}_{j, t}^{Y}\right)^{H}\right]
$$

Since we want to decompose $\nu_{X Y, t}\left(\tau_{j}\right)$, we will make explicit $\widetilde{W}_{j, t}^{X}$ and $\widetilde{W}_{j, t}^{Y}$ as in (2.44) and separate their information along the time, i.e. crossing past $X_{t, T}$ with future $Y_{t, T}$ observations and vice-versa.

$$
\begin{aligned}
& \mathbb{E}\left[\widetilde{W}_{j, t}^{X}\left(\widetilde{W}_{j, t}^{Y}\right)^{H}\right]=\mathbb{E}\left[\left(\sum_{l=0}^{L_{j}-1} \widetilde{h}_{j, l} X_{t-l}\right)\left(\widetilde{W}_{j, t}^{Y}\right)^{H}\right]= \\
= & \mathbb{E}\left[\widetilde{h}_{j, 0} X_{t}\left(\widetilde{W}_{j, t}^{Y}\right)^{H}\right]+\mathbb{E}\left[\widetilde{h}_{j, 1} X_{t-1}\left(\widetilde{W}_{j, t}^{Y}\right)^{H}\right]+\ldots+\mathbb{E}\left[\widetilde{h}_{j, L_{j}-1} X_{t-L_{j}+1}\left(\widetilde{W}_{j, t}^{Y}\right)^{H}\right]= \\
= & \mathbb{E}\left[\widetilde{h}_{j, 0} X_{t}\left(\sum_{l=0}^{L_{j}-1} \widetilde{h}_{j, l} Y_{t-l}\right)^{H}\right]+\mathbb{E}\left[\widetilde{h}_{j, 1} X_{t-1}\left(\sum_{l=0}^{L_{j}-1} \widetilde{h}_{j, l} Y_{t-l}\right)^{H}\right]+\ldots+ \\
+ & \mathbb{E}\left[\widetilde{h}_{j, L_{j}-1} X_{t-L_{j}+1}\left(\sum_{l=0}^{L_{j}-1} \widetilde{h}_{j, l} Y_{t-l}\right)^{H}\right]=
\end{aligned}
$$

Notice that in expression above the term $\mathbb{E}\left(X_{t_{1}} Y_{t_{2}}^{H}\right)$ will appear several times. We want to organize them such that we can replace it by $\mathbb{E}\left(X_{t_{1}} Y_{t_{2}}^{H}\right)=\gamma\left(t_{1}, t_{2}\right)+\mathbb{E}\left(X_{t_{1}}\right) \mathbb{E}\left(Y_{t_{2}}^{H}\right)$ :

$$
\begin{array}{ll}
= & \mathbb{E}\left[\widetilde{h}_{j, 0} X_{t}\left(\widetilde{h}_{j, 0} Y_{t}\right)^{H}\right]+\mathbb{E}\left[\widetilde{h}_{j, 0} X_{t}\left(\widetilde{h}_{j, 1} Y_{t-1}\right)^{H}\right]+\ldots+\mathbb{E}\left[\widetilde{h}_{j, 0} X_{t}\left(\widetilde{h}_{j, L_{j}-1} Y_{t-L_{j}+1}\right)^{H}\right]+ \\
+ & \mathbb{E}\left[\widetilde{h}_{j, 1} X_{t-1}\left(\widetilde{h}_{j, 0} Y_{t}\right)^{H}\right]+\mathbb{E}\left[\widetilde{h}_{j, 1} X_{t-1}\left(\widetilde{h}_{j, 1} Y_{t-1}\right)^{H}\right] \\
& +\ldots+\mathbb{E}\left[\widetilde{h}_{j, 1} X_{t-1}\left(\widetilde{h}_{j, L_{j}-1}\right)^{H} Y_{t-L_{j}+1}\right]+ \\
& +\ldots+
\end{array}
$$




$$
\begin{array}{ll}
+ & \mathbb{E}\left[\widetilde{h}_{j, t-L_{j}+1} X_{t-L_{j}+1}\left(\widetilde{h}_{j, 0} Y_{t}\right)^{H}\right]+\mathbb{E}\left[\widetilde{h}_{j, t-L_{j}+1} X_{t-L_{j}+1}\left(\widetilde{h}_{j, 1} Y_{t-1}\right)^{H}\right]+ \\
& +\ldots+\mathbb{E}\left[\widetilde{h}_{j, t-L_{j}+1} X_{t-L_{j}+1}\left(\widetilde{h}_{j, L_{j}-1} Y_{t-L_{j}+1}\right)^{H}\right]= \\
= & \widetilde{h}_{j, 0}\left(\widetilde{h}_{j, 0}\right)^{H} \mathbb{E}\left[X_{t} Y_{t}^{H}\right]+\widetilde{h}_{j, 0}\left(\widetilde{h}_{j, 1}\right)^{H} \mathbb{E}\left[X_{t} Y_{t-1}^{H}\right]+\ldots+\widetilde{h}_{j, 0}\left(\widetilde{h}_{j, L_{j}-1}\right)^{H} \mathbb{E}\left[X_{t} Y_{t-L_{j}+1}^{H}\right]+ \\
+ & \widetilde{h}_{j, 1}\left(\widetilde{h}_{j, 0}\right)^{H} \mathbb{E}\left[X_{t-1} Y_{t}^{H}\right]+\widetilde{h}_{j, 1}\left(\widetilde{h}_{j, 1}\right)^{H} \mathbb{E}\left[X_{t-1} Y_{t-1}^{H}\right]+\ldots+\widetilde{h}_{j, 1}\left(\widetilde{h}_{j, L_{j}-1}\right)^{H} \mathbb{E}\left[X_{t-1} Y_{t-L_{j}+1}^{H}\right]+ \\
+\ldots+ & \\
+\quad & \widetilde{h}_{j, L_{j}-1}\left(\widetilde{h}_{j, 0}\right)^{H} \mathbb{E}\left[X_{t-L_{j}+1} Y_{t}^{H}\right]+\widetilde{h}_{j, L_{j}-1}\left(\widetilde{h}_{j, 1}\right)^{H} \mathbb{E}\left[X_{t-L_{j}+1} Y_{t-1}^{H}\right]+ \\
& +\ldots+\widetilde{h}_{j, L_{j}-1}\left(\widetilde{h}_{j, L_{j}-1}\right)^{H} \mathbb{E}\left[X_{t-L_{j}+1} Y_{t-L_{j}+1}^{H}\right] .
\end{array}
$$

Now, since $\gamma\left(t_{1}, t_{2}\right)=\mathbb{E}\left(X_{t_{1}} Y_{t_{2}}^{H}\right)-\mathbb{E}\left(X_{t_{1}}\right) \mathbb{E}\left(Y_{t_{2}}^{H}\right)$ we have:

$$
\begin{array}{lll}
= & \widetilde{h}_{j, 0}\left(\widetilde{h}_{j, 0}\right)^{H}\left(\gamma(t, t)+\mu_{X}(t / T) \mu_{Y}(t / T)\right)+\widetilde{h}_{j, 0}\left(\widetilde{h}_{j, 1}\right)^{H}\left(\gamma(t, t-1)+\mu_{X}(t / T) \mu_{Y}((t-1) / T)\right) \\
& +\ldots+\widetilde{h}_{j, 0}\left(\widetilde{h}_{j, L_{j}-1}\right)^{H}\left(\gamma\left(t, t-L_{j}+1\right)+\mu_{X}(t / T) \mu_{Y}\left(\left(t-L_{j}+1\right) / T\right)\right)+ \\
+ & \widetilde{h}_{j, 1}\left(\widetilde{h}_{j, 0}\right)^{H}\left(\gamma(t-1, t)+\mu_{X}((t-1) / T) \mu_{Y}(t / T)\right)+ \\
+ & \widetilde{h}_{j, 1}\left(\widetilde{h}_{j, 1}\right)^{H}\left(\gamma(t-1, t-1)+\mu_{X}((t-1) / T) \mu_{Y}((t-1) / T)\right)+ \\
& +\ldots+\widetilde{h}_{j, 1}\left(\widetilde{h}_{j, L_{j}-1}\right)^{H}\left(\gamma\left(t-1, t-L_{j}+1\right)+\mu_{X}((t-1) / T) \mu_{Y}\left(\left(t-L_{j}+1\right) / T\right)\right)+ \\
+\ldots+ & \\
+\quad & \widetilde{h}_{j, L_{j}-1}\left(\widetilde{h}_{j, 0}\right)^{H}\left(\gamma\left(t-L_{j}+1, t\right)+\mu_{X}\left(\left(t-L_{j}+1\right) / T\right) \mu_{Y}(t / T)\right)+ \\
& +\widetilde{h}_{j, L_{j}-1}\left(\widetilde{h}_{j, 1}\right)^{H}\left(\gamma\left(t-L_{j}+1, t-1\right)+\mu_{X}\left(\left(t-L_{j}+1\right) / T\right) \mu_{Y}((t-1) / T)\right)+ \\
& +\ldots+\widetilde{h}_{j, L_{j}-1}\left(\widetilde{h}_{j, L_{j}-1}\right)^{H}\left(\gamma\left(t-L_{j}+1, t-L_{j}+1\right)+\right. \\
& \left.\mu_{X}\left(\left(t-L_{j}+1\right) / T\right) \mu_{Y}\left(\left(t-L_{j}+1\right) / T\right)\right)=
\end{array}
$$

Now, for $\gamma\left(t_{1}, t_{2}\right)$ we have the following possible situations:

- $t_{1}<t_{2}: X_{t_{1}, T}$ is located in time before $Y_{t_{2}, T}$;

- $t_{1}=t_{2}: X_{t_{1}, T}$ and $Y_{t_{2}, T}$ are simultaneous;

- $t_{1}>t_{2}: X_{t_{1}, T}$ is located in time ahead $Y_{t_{2}, T}$.

We will then separate the terms according to the three cases above:

$$
\begin{aligned}
& =\sum_{l=0}^{L_{j}-1} \sum_{l^{\prime}=0}^{L_{j}-1} \widetilde{h}_{j, l}\left(\widetilde{h}_{j, l^{\prime}}\right)^{H} \mu_{X}((t-l) / T) \mu_{Y}\left(\left(t-l^{\prime}\right) / T\right)+\sum_{l=1}^{L_{j}-1} \sum_{l^{\prime}=0}^{l-1} \widetilde{h}_{j, l}\left(\widetilde{h}_{j, l^{\prime}}\right)^{H}\left(\gamma\left(t-l, t-l^{\prime}\right)\right)+ \\
+ & \left.\sum_{l=0}^{L_{j}-1} \widetilde{h}_{j, l}\left(\widetilde{h}_{j, l}\right)^{H}(\gamma(t-l, t-l))+\sum_{l=0}^{L_{j}-2} \sum_{l^{\prime}=l+1}^{L_{j}-1} \widetilde{h}_{j, l} \widetilde{h}_{j, l^{\prime}}\right)^{H}\left(\gamma\left(t-l, t-l^{\prime}\right)\right)= \\
& =\widetilde{W}_{j, t}\left(\mu_{X}(u)\right) \widetilde{W}_{j, t}\left(\mu_{Y}(u)\right)+\sum_{l=1}^{L_{j}-1} \sum_{l^{\prime}=0}^{l-1} \widetilde{h}_{j, l}\left(\widetilde{h}_{j, l^{\prime}}\right)^{H}\left(\gamma\left(t-l, t-l^{\prime}\right)\right)+
\end{aligned}
$$




$$
+\sum_{l=0}^{L_{j}-1} \widetilde{h}_{j, l}\left(\widetilde{h}_{j, l}\right)^{H}(\gamma(t-l, t-l))+\sum_{l=0}^{L_{j}-2} \sum_{l^{\prime}=l+1}^{L_{j}-1} \widetilde{h}_{j, l}\left(\widetilde{h}_{j, l^{\prime}}\right)^{H}\left(\gamma\left(t-l, t-l^{\prime}\right)\right) .
$$

Proof of Proposition 3.2. We present the expectation calculation for $\widehat{F}_{X Y, t}\left(\tau_{j}\right)$. The result for $\widehat{B}_{X Y, t}\left(\tau_{j}\right)$ is analogous.

$$
\begin{gathered}
\mathbb{E}\left[\widehat{F}_{X Y, t}\left(\tau_{j}\right)\right]=\mathbb{E}\left\{\sum_{l=1}^{L_{j}-1} \sum_{l^{\prime}=0}^{l-1} \widetilde{h}_{j, l}\left(\tilde{h}_{j, l^{\prime}}\right)^{H} \hat{c}_{T}\left(\frac{t-l}{T}, l^{\prime}-l\right)\right\}= \\
\sum_{l=1}^{L_{j}-1} \sum_{l^{\prime}=0}^{l-1} \widetilde{h}_{j, l}\left(\tilde{h}_{j, l^{\prime}}\right)^{H} \mathbb{E}\left[\hat{c}_{T}\left(\frac{t-l}{T}, l^{\prime}-l\right)\right]=\sum_{l=1}^{L_{j}-1} \sum_{l^{\prime}=0}^{l-1} \widetilde{h}_{j, l}\left(\tilde{h}_{j, l^{\prime}}\right)^{H}\left[c\left(\frac{t-l}{T}, l-l^{\prime}\right)+\right. \\
+(1 / 2) b_{T}^{2} \int x^{2} K(x) d x\left(\frac{\partial^{2}}{\partial u^{2}} c\left(\frac{t-l}{T}, l-l^{\prime}\right)\right)+o\left(b_{T}^{2}\right)+O\left(\frac{1}{b_{T} T}\right)= \\
=F_{X Y, t}\left(\tau_{j}\right)+\sum_{l=1}^{L_{j}-1} \sum_{l^{\prime}=0}^{l-1} \widetilde{h}_{j, l}\left(\tilde{h}_{j, l^{\prime}}\right)^{H} \frac{1}{2} b_{T}^{2} \int x^{2} K(x) d x \frac{\partial^{2}}{\partial t^{2}} c\left(t+l, l-l^{\prime}\right)+o\left(b_{T}^{2}\right)+O\left(\frac{1}{b_{T} T}\right)= \\
=F_{X Y, t}\left(\tau_{j}\right)+\frac{1}{2} b_{T}^{2} \int x^{2} K(x) d x \sum_{l=1}^{L_{j}-1} \sum_{l^{\prime}=0}^{l-1} \widetilde{h}_{j, l}\left(\tilde{h}_{j, l^{\prime}}\right)^{H} \frac{\partial^{2}}{\partial t^{2}} c\left(t+l, l-l^{\prime}\right)+o\left(b_{T}^{2}\right)+O\left(\frac{1}{b_{T} T}\right) .
\end{gathered}
$$

\section{Proof of Proposition 3.3.}

$$
\begin{gathered}
\operatorname{Var}\left[\widehat{F}_{X Y, t}\left(\tau_{j}\right)\right]=\operatorname{Var}\left\{\sum_{l=1}^{L_{j}-1} \sum_{l^{\prime}=0}^{l-1} \widetilde{h}_{j, l} \tilde{h}_{j, l^{\prime}}^{H} \hat{c}_{T}\left(\frac{t-l}{T}, l^{\prime}-l\right)\right\} \leq \\
\operatorname{Var}\left\{\sum_{l=1}^{L_{j}-1} \sum_{l^{\prime}=0}^{l-1}\left|\widetilde{h}_{j, l}\right| \cdot\left|\tilde{h}_{j, l^{\prime}}^{H}\right| \hat{c}_{T}\left(\frac{t-l}{T}, l^{\prime}-l\right)\right\} \leq \\
\operatorname{Var}\left\{\sum_{l=1}^{L_{j}-1} \sum_{l^{\prime}=0}^{l-1} \max _{l}\left\{\left|\widetilde{h}_{j, l}\right|\right\}^{2} \hat{c}_{T}\left(\frac{t-l}{T}, l^{\prime}-l\right)\right\}= \\
\operatorname{Var}\left\{\sum_{l=1}^{L_{j}-1} \sum_{l^{\prime}=0}^{l-1} \max _{l}\left\{\left|\widetilde{h}_{j, l}\right|\right\}^{2} \frac{1}{b_{T} T} \sum_{s} K\left(\frac{t-l-s+\left(l^{\prime}-l\right) / 2}{b_{T} T}\right) X_{s} Y_{s+l^{\prime}-l}\right\}= \\
\frac{\max _{l}\left\{\left|\widetilde{h}_{j, l}\right|\right\}^{4}}{\left(b_{T} T\right)^{2}} \operatorname{Var}\left\{\sum_{l=1}^{L_{j}-1} \sum_{l^{\prime}=0} \sum_{s} K\left(\frac{t-l-s+\left(l^{\prime}-l\right) / 2}{b_{T} T}\right) X_{s} Y_{s+l^{\prime}-l}\right\}=\star
\end{gathered}
$$

Using Epanechnikov kernel: $K(x)=-6 x^{2}+3 / 2$ is maximum when $x=0 \Rightarrow K(0)=3 / 2$. Also, $K\left(\frac{t-l-s+\left(l^{\prime}-l\right) / 2}{b_{T} T}\right) \Rightarrow s \in S_{t}=\left\{s: t-l-\left(l^{\prime}-l\right) / 2-b_{T} T / 2 \leq s \leq t-l-\left(l^{\prime}-l\right) / 2+b_{T} T / 2\right\}$. 


$$
\begin{aligned}
\star \leq \frac{\max _{l}\left\{\left|\widetilde{h}_{j, l}\right|\right\}^{4}}{\left(b_{T} T\right)^{2}} \operatorname{Var}\left\{\sum_{l=1}^{L_{j}-1} \sum_{l^{\prime}=0}^{l-1} \sum_{s \in S_{t}} 3 / 2 X_{s} Y_{s+l^{\prime}-l}\right\}= \\
=\frac{9 \max _{l}\left\{\left|\widetilde{h}_{j, l}\right|\right\}^{4}}{4\left(b_{T} T\right)^{2}} \operatorname{Var}\left\{\sum_{l=1}^{L_{j}-1} \sum_{l^{\prime}=0}^{l-1} \sum_{s \in S_{t}} X_{s} Y_{s+l^{\prime}-l}\right\} .
\end{aligned}
$$

Since we have:

\begin{tabular}{cll}
\hline$l$ & $l^{\prime}$ & $l^{\prime}-l$ \\
\hline 1 & 0 & -1 \\
2 & 0,1 & $-2,-1$ \\
3 & $0,1,2$ & $-3,-2,-1$ \\
$\vdots$ & $\vdots$ & $\vdots$ \\
$L_{j}-1$ & $0,1,2, \ldots, L_{j}-2$ & $-L_{j}+1, \ldots,-2,-1$ \\
\hline
\end{tabular}

then

$$
\begin{gathered}
\operatorname{Var}\left[\widehat{F}_{X Y, t}\left(\tau_{j}\right)\right] \leq \frac{9 \max _{l}\left\{\left|\widetilde{h}_{j, l}\right|\right\}^{4}}{4\left(b_{T} T\right)^{2}} \operatorname{Var}\left\{\left(L_{j}-1\right) \sum_{s \in S_{t}} X_{s} Y_{s-1}+\left(L_{j}-2\right) \sum_{s \in S_{t}} X_{s} Y_{s-2}+\cdots+\right. \\
\left.+(2) \sum_{s \in S_{t}} X_{s} Y_{s-L_{j}+2}+\sum_{s \in S_{t}} X_{s} Y_{s-L_{j}+1}\right\} \\
\Rightarrow \operatorname{Var}\left[\widehat{F}_{X Y, t}\left(\tau_{j}\right)\right] \leq \frac{9 \max _{l}\left\{\left|\widetilde{h}_{j, l}\right|\right\}^{4}}{4\left(b_{T} T\right)^{2}} \operatorname{Var}\left\{\sum_{l^{*}=1}^{L_{j}-1}\left(L_{j}-l^{*}\right) \sum_{s \in S_{t}} X_{s} Y_{s-l^{*}}\right\} .
\end{gathered}
$$

Now, since $X_{t}$ and $Y_{t}$ fulfill Definition 2.6 then the quantity $\operatorname{Var}\left\{\sum_{l}\left(L_{j}-l\right) \sum_{s} X_{s} Y_{s-l}\right\}$ is finite and therefore (B.1) goes to zero as $T$ goes to infinity.

The proof for $\operatorname{Var}\left[\widehat{B}_{X Y, t}\left(\tau_{j}\right)\right]$ is analogous.

Proof of Proposition 4.1. For one particular component of $\mathbf{X}_{t, T}$ in (4.3), say $X_{t, T}^{(1)}$, we have:

$$
\begin{aligned}
X_{t, T}^{(1)} & =\mu_{1}(u)+\underbrace{\sum_{k=1}^{D} a_{1, k}^{(1)}(u) X_{t-1, T}^{(k)}}_{\operatorname{lag}(1) \text { of all components } X_{1}, \ldots, X_{D}}+\cdots+\underbrace{\sum_{k=1}^{D} a_{1, k}^{(p)}(u) X_{t-p, T}^{(k)}}_{\operatorname{lag}(\mathrm{p}) \text { of all components } X_{1}, \ldots, X_{D}}+\epsilon_{\text {impact of } \mathcal{F}_{1}(t) \text { in } X_{1}}^{\sum_{m=1}^{p} a_{1,1}^{(m)}(u) X_{t-m, T}^{(1)}}+\cdots+\underbrace{\sum_{k=1}^{p} a_{1, D}^{(m)}(u) X_{t-m, T}^{(D)}}_{\text {impact of } \mathcal{F}_{D}(t) \text { in } X_{1}}+\epsilon_{1}(t) \\
& =\mu_{1}(u)=
\end{aligned}
$$

where $\mathcal{F}_{i}(t), i=1, \ldots, D$ is all past information of the component $X_{i}(t), t=1, \ldots, t-1$. This representation allows us to evaluate, in separated terms, the impact of each component of $\mathbf{X}_{t, T}$ into $X_{t, T}^{(1)}$.

Now, as in Proposition 3.1, consider $t \in \Upsilon=\left\{t \mid t \geq L_{j}\right.$ and $\left.t \leq T-L_{j}, \forall j\right\}$ then we can ignore the 
modulo operation in the MODWT. Hence, for $X_{t, T}^{(1)}$ the $\mathrm{j}$-th wavelet coefficients are given by

$$
\begin{aligned}
W_{j, t}^{(1)}= & \sum_{l=0}^{L_{j}-1} \widetilde{h}_{j, l} X_{t-l M o d}^{(1)}=\sum_{l=0}^{L_{j}-1} \widetilde{h}_{j, l} X_{t-l}^{(1)}=\sum_{l=0}^{L_{j}-1} \widetilde{h}_{j, l} \mu_{1}((t-l) / T)+\sum_{l=0}^{L_{j}-1} \widetilde{h}_{j, l}\left(\sum_{m=1}^{p} a_{1,1}^{(m)}(u) X_{t-l-m, T}^{(1)}\right)+ \\
& +\sum_{l=0}^{L_{j}-1} \widetilde{h}_{j, l}\left(\sum_{m=1}^{p} a_{1,2}^{(m)}(u) X_{t-l-m, T}^{(2)}\right)+\cdots+\sum_{l=0}^{L_{j}-1} \widetilde{h}_{j, l}\left(\sum_{m=1}^{p} a_{1, D}^{(m)}(u) X_{t-l-m, T}^{(D)}\right)+\sum_{l=0}^{L_{j}-1} \widetilde{h}_{j, l} \epsilon_{1}(t-l)= \\
= & \widetilde{W}_{j, t}\left(\mu_{1}(u)\right)+\mathcal{W}_{j, t}\left(\mathcal{F}_{1,1}(t)\right)+\mathcal{W}_{j, t}\left(\mathcal{F}_{1,2}(t)\right)+\cdots+\mathcal{W}_{j, t}\left(\mathcal{F}_{1, D}(t)\right)+\widetilde{W}_{j, t}\left(\epsilon_{1}(t)\right)
\end{aligned}
$$

So, from (B.2) we have that the MODWT of $X_{t, T}^{(1)}$ is a sum of MODWTs of its mean, the error and all the past information of each component of $\mathbf{X}_{t, T}$, including $X_{t, T}^{(1)}$.

Analogously, for $X_{t, T}^{(2)}$ we have:

$$
\widetilde{W}_{j, t}^{(2)}=\widetilde{W}_{j, t}\left(\mu_{2}(u)\right)+\mathcal{W}_{j, t}\left(\mathcal{F}_{2,1}(t)\right)+\mathcal{W}_{j, t}\left(\mathcal{F}_{2,2}(t)\right)+\cdots+\mathcal{W}_{j, t}\left(\mathcal{F}_{2, D}(t)\right)+\widetilde{W}_{j, t}\left(\epsilon_{2}(t)\right)
$$

Now, from equations (B.3) and (B.4) let's calculate $\nu\left(X^{(1)}, X^{(2)}, \tau_{j}\right)=\operatorname{Cov}\left(\widetilde{W}_{j, t}^{(1)}, \widetilde{W}_{j, t}^{(2)}\right)$ :

$$
\begin{aligned}
& \nu\left(X^{(1)}, X^{(2)}, \tau_{j}\right)=\operatorname{Cov}\left(\widetilde{W}_{j, t}^{(1)}, \widetilde{W}_{j, t}^{(2)}\right)=\mathbb{E}\left(\widetilde{W}_{j, t}^{(1)} \cdot\left(\widetilde{W}_{j, t}^{(2)}\right)^{H}\right)-\mathbb{E}\left(\widetilde{W}_{j, t}^{(1)}\right) \mathbb{E}\left(\left(\widetilde{W}_{j, t}^{(2)}\right)^{H}\right)^{=0}= \\
& =\mathbb{E}\left\{\left[\widetilde{W}_{j, t}\left(\mu_{1}(u)\right)+\mathcal{W}_{j, t}\left(\mathcal{F}_{1,1}(t)\right)+\cdots+\mathcal{W}_{j, t}\left(\mathcal{F}_{1, D}(t)\right)+\widetilde{W}_{j, t}\left(\epsilon_{1}(t)\right)\right] \times\right. \\
& \left.\left[\widetilde{W}_{j, t}\left(\mu_{2}(u)\right)+\mathcal{W}_{j, t}\left(\mathcal{F}_{2,1}(t)\right)+\cdots+\mathcal{W}_{j, t}\left(\mathcal{F}_{2, D}(t)\right)+\widetilde{W}_{j, t}\left(\epsilon_{2}(t)\right)\right]\right\}=
\end{aligned}
$$

Now, if we calculate the cross multiplication and join the common factors we have:

$$
\begin{aligned}
& =\mathbb{E}\left\{\left[\widetilde{W}_{j, t}\left(\mu_{1}(u)\right)+\sum_{i=1}^{D} \mathcal{W}_{j, t}\left(\mathcal{F}_{1, i}(t)\right)+\widetilde{W}_{j, t}\left(\epsilon_{1}(t)\right)\right]\left[\widetilde{W}_{j, t}\left(\mu_{2}(u)\right)+\sum_{i=1}^{D} \mathcal{W}_{j, t}\left(\mathcal{F}_{2, i}(t)\right)+\widetilde{W}_{j, t}\left(\epsilon_{2}(t)\right)\right]\right\}= \\
& =\mathbb{E}\left\{\widetilde{W}_{j, t}\left(\mu_{1}(u)\right) \widetilde{W}_{j, t}\left(\mu_{2}(u)\right)+\right. \\
& +\widetilde{W}_{j, t}\left(\mu_{1}(u)\right)\left[\sum_{i=1}^{D} \mathcal{W}_{j, t}\left(\mathcal{F}_{2, i}(t)\right)\right]+\widetilde{W}_{j, t}\left(\mu_{2}(u)\right)\left[\sum_{i=1}^{D} \mathcal{W}_{j, t}\left(\mathcal{F}_{1, i}(t)\right)\right]+ \\
& +\widetilde{W}_{j, t}\left(\mu_{1}(u)\right) \widetilde{W}_{j, t}\left(\epsilon_{2}(t)\right)+\widetilde{W}_{j, t}\left(\epsilon_{1}(t)\right) \widetilde{W}_{j, t}\left(\mu_{2}(u)\right)+\widetilde{W}_{j, t}\left(\epsilon_{1}(t)\right) \widetilde{W}_{j, t}\left(\epsilon_{2}(t)\right)+ \\
& +\widetilde{W}_{j, t}\left(\epsilon_{1}(t)\right)\left[\sum_{i=1}^{D} \mathcal{W}_{j, t}\left(\mathcal{F}_{2, i}(t)\right)\right]+\widetilde{W}_{j, t}\left(\epsilon_{2}(t)\right)\left[\sum_{i=1}^{D} \mathcal{W}_{j, t}\left(\mathcal{F}_{1, i}(t)\right)\right]+ \\
& \left.+\mathcal{W}_{j, t}\left(\mathcal{F}_{1,1}(t)\right)\left[\sum_{i=1}^{D} \mathcal{W}_{j, t}\left(\mathcal{F}_{2, i}(t)\right)\right]+\cdots+\mathcal{W}_{j, t}\left(\mathcal{F}_{1, D}(t)\right)\left[\sum_{i=1}^{D} \mathcal{W}_{j, t}\left(\mathcal{F}_{2, i}(t)\right)\right]\right\} \\
& =\underbrace{\mathbb{E}\left\{\widetilde{W}_{j, t}\left(\mu_{1}(u)\right) \widetilde{W}_{j, t}\left(\mu_{2}(u)\right)\right\}}_{\text {I }}+ \\
& +\underbrace{\mathbb{E}\left\{\widetilde{W}_{j, t}\left(\mu_{1}(u)\right)\left[\sum_{i=1}^{D} \mathcal{W}_{j, t}\left(\mathcal{F}_{2, i}(t)\right)\right]\right\}}_{\text {II }}+\underbrace{\mathbb{E}\left\{\widetilde{W}_{j, t}\left(\mu_{2}(u)\right)\left[\sum_{i=1}^{D} \mathcal{W}_{j, t}\left(\mathcal{F}_{1, i}(t)\right)\right]\right\}}_{\text {III }}+
\end{aligned}
$$




$$
\begin{aligned}
& +\underbrace{\mathbb{E}\left\{\widetilde{W}_{j, t}\left(\epsilon_{1}(t)\right) \widetilde{W}_{j, t}\left(\mu_{2}(u)\right)\right\}}_{\text {IV }\left\{\widetilde{W}_{j, t}\left(\mu_{1}(u)\right) \widetilde{W}_{j, t}\left(\epsilon_{2}(t)\right)\right\}}+\underbrace{\mathbb{E}\left\{\widetilde{W}_{j, t}\left(\epsilon_{1}(t)\right) \widetilde{W}_{j, t}\left(\epsilon_{2}(t)\right)\right\}}_{\text {V }}+ \\
& +\underbrace{\mathbb{E}\left\{\widetilde{W}_{j, t}\left(\epsilon_{1}(t)\right)\left[\sum_{i=1}^{D} \mathcal{W}_{j, t}\left(\mathcal{F}_{2, i}(t)\right)\right]\right\}}_{\text {VII }}+\underbrace{\mathbb{E}\left\{\widetilde{W}_{j, t}\left(\epsilon_{2}(t)\right)\left[\sum_{i=1}^{D} \mathcal{W}_{j, t}\left(\mathcal{F}_{1, i}(t)\right)\right]\right\}}_{\text {VIII }}+ \\
& +\underbrace{\mathbb{E}\left\{\mathcal{W}_{j, t}\left(\mathcal{F}_{1,1}(t)\right)\left[\sum_{i=1}^{D} \mathcal{W}_{j, t}\left(\mathcal{F}_{2, i}(t)\right)\right]+\cdots+\mathcal{W}_{j, t}\left(\mathcal{F}_{1, D}(t)\right)\left[\sum_{i=1}^{D} \mathcal{W}_{j, t}\left(\mathcal{F}_{2, i}(t)\right)\right]\right\}}_{\text {IX }}
\end{aligned}
$$

As we did with DWC in Chapter 3, we want to establish a causal relation between past observations of $X_{t, T}^{(1)}$ and future observations of $X_{t, T}^{(2)}$, and vice-versa. So, for each one of the terms I to $\mathbf{I X}$ above, we aim a simplification that explicits these relations.

- Calculating I:

$$
\mathbb{E}\left\{\widetilde{W}_{j, t}\left(\mu_{1}(u)\right) \widetilde{W}_{j, t}\left(\mu_{2}(u)\right)\right\}=\widetilde{W}_{j, t}\left(\mu_{1}(u)\right) \widetilde{W}_{j, t}\left(\mu_{2}(u)\right)
$$

i.e., this term is equivalent to the product of the MODWT of $\mu_{1}(u)$ and $\mu_{1}(u)$.

\section{- Calculating II-VI:}

The terms II-VI are all equal to zero.

$$
\begin{aligned}
\text { II: } & \mathbb{E}\left\{\widetilde{W}_{j, t}\left(\mu_{1}(u)\right)\left[\sum_{i=1}^{D} \mathcal{W}_{j, t}\left(\mathcal{F}_{2, i}(t)\right)\right]\right\}=\widetilde{W}_{j, t}\left(\mu_{1}(u)\right) \sum_{i=1}^{D} \mathbb{E}\left\{\mathcal{W}_{j, t}\left(\mathcal{F}_{2, i}(t)\right)\right\}= \\
= & \widetilde{W}_{j, t}\left(\mu_{1}(u)\right) \sum_{i=1}^{D} \mathbb{E}\{\underbrace{\sum_{l=0}^{L_{j}-1} \widetilde{h}_{j, l}\left(\sum_{m=1}^{p} a_{1, i}^{(m)}(u) X_{t-l-m, T}^{(i)}\right)}_{\text {(IIa) }}\}
\end{aligned}
$$

Consider the process $Q_{t}=\left(\sum_{m=1}^{p} a_{1, i}^{(m)}(u) X_{t-m, T}^{(i)}\right)$. Then its MODWT will be equal to (IIa) and has zero mean for every $i$ in $\{1, \ldots, D\}$. Therefore we have:

$$
\text { II: } \widetilde{W}_{j, t}\left(\mu_{1}(u)\right) \sum_{i=1}^{D} \mathbb{E}\left\{\sum_{l=0}^{L_{j}-1} \widetilde{h}_{j, l}\left(\sum_{m=1}^{p} a_{1, i}^{(m)}(u) X_{t-l-m, T}^{(i)}\right)\right\}=0
$$

Analogously, the next quantities are equal to zero:

$$
\text { III: } \quad \mathbb{E}\left\{\widetilde{W}_{j, t}\left(\mu_{2}(u)\right)\left[\sum_{i=1}^{D} \mathcal{W}_{j, t}\left(\mathcal{F}_{1, i}(t)\right)\right]\right\}=\widetilde{W}_{j, t}\left(\mu_{2}(u)\right) \sum_{i=1}^{D} \mathbb{E}\left\{\mathcal{W}_{j, t}\left(\mathcal{F}_{1, i}(t)\right)\right\}=0
$$


$\mathrm{IV}: \quad \mathbb{E}\left\{\widetilde{W}_{j, t}\left(\mu_{1}(u)\right) \widetilde{W}_{j, t}\left(\epsilon_{2}(t)\right)\right\}=\widetilde{W}_{j, t}\left(\mu_{1}(t)\right) \mathbb{E}\left\{\widetilde{W}_{j, t}\left(\epsilon_{2}(t)\right)\right\}=0$

$\mathrm{V}: \quad \mathbb{E}\left\{\widetilde{W}_{j, t}\left(\epsilon_{1}(t)\right) \widetilde{W}_{j, t}\left(\mu_{2}(u)\right)\right\}=\widetilde{W}_{j, t}\left(\mu_{2}(t)\right) \mathbb{E}\left\{\widetilde{W}_{j, t}\left(\epsilon_{1}(t)\right)\right\}=0$

VI: $\quad \mathbb{E}\left\{\widetilde{W}_{j, t}\left(\epsilon_{1}(t)\right) \widetilde{W}_{j, t}\left(\epsilon_{2}(t)\right)\right\}=\mathbb{E}\left\{\widetilde{W}_{j, t}\left(\epsilon_{1}(t)\right)\right\} \mathbb{E}\left\{\widetilde{W}_{j, t}\left(\epsilon_{2}(t)\right)\right\}=0$

- Calculating VII-VIII:

First notice that the terms VII and VIII represent relations between past random errors and each of the components $X^{(1)}$ and $X^{(2)}$. Therefore their simplification is due only to show they do not have terms that will contribute to the proposition of ppDWC. Thus, to prove that first consider the following representation for a $\operatorname{VAR}(p)$ model in Lütkepohl (2007), i.e., the equation (4.3) can be written in $\operatorname{VAR}(1)$ form as follows:

$$
\mathbf{Y}_{t}=\nu(u)+\mathbf{A}(u) \mathbf{Y}_{t-1}+U(t)
$$

with

$$
\begin{aligned}
& \mathbf{Y}_{t}=\left[\begin{array}{c}
\mathbf{X}_{t, T} \\
\mathbf{X}_{t-1, T} \\
\vdots \\
\mathbf{X}_{t-p+1, T}
\end{array}\right]_{(p D \times 1)}, \quad \nu(u)=\left[\begin{array}{c}
\boldsymbol{\mu}(u) \\
0 \\
\vdots \\
0
\end{array}\right]_{(p D \times 1)} \\
& \mathbf{A}(u)=\left[\begin{array}{ccccc}
\mathbf{A}_{1}(u) & \mathbf{A}_{2}(u) & \ldots & \mathbf{A}_{p-1}(u) & \mathbf{A}_{p}(u) \\
I_{D} & 0_{D} & \ldots & 0_{D} & 0_{D} \\
0_{D} & I_{D} & \ldots & 0_{D} & 0_{D} \\
\vdots & \vdots & \ddots & \vdots & \vdots \\
0_{D} & 0_{D} & \ldots & I_{D} & 0_{D}
\end{array}\right]_{(p D \times p D)} \quad, \quad U(t)=\left[\begin{array}{c}
\boldsymbol{\epsilon}(t) \\
0 \\
\vdots \\
0
\end{array}\right]_{(p D \times 1)} .
\end{aligned}
$$

Under the stability condition, i.e.

$$
\operatorname{det}\left(I_{D}-\mathbf{A}(u) z\right)=\operatorname{det}\left(I_{D}-\mathbf{A}_{1}(u) z-\mathbf{A}_{2}(u) z^{2}-\cdots-\mathbf{A}_{p}(u) z^{p}\right) \neq 0, \text { for }|z| \leq 1,
$$

we can represent this process as a $M A(\infty)$ :

$$
\mathbf{Y}_{t}=\mu(u)+\sum_{i=0}^{\infty} \mathbf{A}^{i}(u) U(t-i)
$$


On other hand we have in expression VIII the term below, that can be written using (B.5).

$$
\begin{aligned}
& \sum_{i=1}^{D} \mathcal{W}_{j, t}\left(\mathcal{F}_{1, i}(t)\right)=\sum_{i=1}^{D} \sum_{l=0}^{L_{j}-1} \widetilde{h}_{j, l}\left(\sum_{m=1}^{p} a_{1, i}^{(m)}(u) X_{t-l-m}^{(i)}\right)=\sum_{l=0}^{L_{j}-1} \widetilde{h}_{j, l}\left(\sum_{i=1}^{D} \sum_{m=1}^{p} a_{1, i}^{(m)}(u) X_{t-l-m}^{(i)}\right)= \\
& =\sum_{l=0}^{L_{j}-1} \widetilde{h}_{j, l}\left(\sum_{m=1}^{p} a_{1}^{(m)}(u) \mathbf{X}_{t-l-m}\right)=\sum_{l=0}^{L_{j}-1} \widetilde{h}_{j, l}\left[\begin{array}{llll}
a_{1}^{(1)}(u) & a_{1}^{(2)}(u) & \ldots & a_{1}^{(p)}(u)
\end{array}\right]\left[\begin{array}{c}
\mathbf{X}_{t-l-1, T} \\
\mathbf{X}_{t-l-2, T} \\
\vdots \\
\mathbf{X}_{t-l-p, T}
\end{array}\right]= \\
& =\sum_{l=0}^{L_{j}-1} \widetilde{h}_{j, l}\left[\begin{array}{llll}
a_{1}^{(1)}(u) & a_{1}^{(2)}(u) & \ldots & a_{1}^{(p)}(u)
\end{array}\right] \mathbf{Y}_{t-l}= \\
& =\left[\begin{array}{llll}
a_{1}^{(1)}(u) & a_{1 \bullet}^{(2)}(u) & \ldots & a_{1}^{(p)}(u)
\end{array}\right]\left[\begin{array}{l}
\sum_{l=0}^{L_{j}-1} \widetilde{h}_{j, l}\left(\mathbf{Y}_{t-l}\right)
\end{array}\right]
\end{aligned}
$$

Using that expression, the term VIII can be written as:

$$
\begin{aligned}
& \mathbb{E}\left\{\widetilde{W}_{j, t}\left(\epsilon_{2}(t)\right)\left[\sum_{i=1}^{D} \mathcal{W}_{j, t}\left(\mathcal{F}_{1, i}(t)\right)\right]\right\}= \\
& =\mathbb{E}\left\{\left[\sum_{l=0}^{L_{j}-1} \widetilde{h}_{j, l} \epsilon_{2}(t-l)\right]\left[\left[a_{1}^{(1)}(u), a_{1}^{(2)}(u), \ldots, a_{1}^{(p)}(u)\right]\left[\sum_{l=0}^{L_{j}-1} \widetilde{h}_{j, l}\left(\mathbf{Y}_{t-l}\right)\right]\right]\right\}= \\
& =\mathbb{E}\left\{\left[\tilde{h}_{j, 0}, \ldots, \tilde{h}_{j, L_{0}-1}\right]\left[\begin{array}{c}
\epsilon_{2}(t) \\
\epsilon_{2}(t-1) \\
\vdots \\
\epsilon_{2}\left(t-L_{j}+1\right)
\end{array}\right]\left[a_{1 \bullet}^{(1)}(u), \ldots, a_{1}^{(p)}(u)\right]\left[\mathbf{Y}_{t}, \ldots, \mathbf{Y}_{t-L_{j}+1}\right]\left[\begin{array}{c}
\tilde{h}_{j, 0} \\
\vdots \\
\tilde{h}_{j, L_{0}-1}
\end{array}\right]\right\}= \\
& =\mathbb{E}\left\{\tilde{\mathbf{h}}_{\mathbf{j}}^{\top} \boldsymbol{\epsilon}_{\mathbf{2}}(t)\left[A_{1}^{*}\right]\left[\mathbf{Y}_{t}, \ldots, \mathbf{Y}_{t-L_{j}+1}\right] \tilde{\mathbf{h}}_{\mathbf{j}}\right\}=\tilde{\mathbf{h}}_{\mathbf{j}}^{\top} \mathbb{E}\left\{\boldsymbol{\epsilon}_{\mathbf{2}}(t)\left[A_{1}^{*}\right]\left[\mathbf{Y}_{t}, \ldots, \mathbf{Y}_{t-L_{j}+1}\right]\right\} \tilde{\mathbf{h}}_{\mathbf{j}} .
\end{aligned}
$$

For simplicity, we omit the index (u) of matrices $A_{1}^{*}$ and $\mathbf{A}^{i}$. Using (B.6) we can show that the term VIII depends only on $\sigma($.$) and the coefficients of the tv-VAR(p). An analogous result$ is valid for term VII.

$$
\begin{aligned}
& \mathbb{E}\left\{\boldsymbol{\epsilon}_{\mathbf{2}}(t)\left[A_{1}^{*}\right]\left[\mathbf{Y}_{t}, \ldots, \mathbf{Y}_{t-L_{j}+1}\right]\right\} \mathbb{E}\left\{\boldsymbol{\epsilon}_{\mathbf{2}}(t)\left[A_{1}^{*}\right]\left[\mathbf{Y}_{t}, \ldots, \mathbf{Y}_{t-L_{j}+1}\right]\right\}= \\
= & \mathbb{E}\left\{\boldsymbol{\epsilon}_{\mathbf{2}}(t)\left[A_{1}^{*}\right]\left[\left(\mu(u)+\sum_{i=0}^{\infty} \mathbf{A}^{i} U(t-i)\right), \ldots,\left(\mu\left(\frac{t-L_{j}+1}{T}\right)+\sum_{i=0}^{\infty} \mathbf{A}^{i} U\left(t-L_{j}+1-i\right)\right)\right]\right\} \\
= & \mathbb{E}\left\{\left[\begin{array}{ccc}
\epsilon_{2}(t) A_{1}^{*} \sum_{i=0}^{\infty} \mathbf{A}^{i} U(t-i) & \vdots & \epsilon_{2}(t) A_{1}^{*} \sum_{i=0}^{\infty} \mathbf{A}^{i} U\left(t-L_{j}+1-i\right)= \\
\vdots & \ddots & \vdots \\
\epsilon_{2}\left(t-L_{j}+1\right) A_{1}^{*} \sum_{i=0}^{\infty} \mathbf{A}^{i} U(t-i) & \vdots & \epsilon_{2}\left(t-L_{j}+1\right) A_{1}^{*} \sum_{i=0}^{\infty} \mathbf{A}^{i} U\left(t-L_{j}+1-i\right)
\end{array}\right]\right\}= \\
= & {\left[\begin{array}{ccc}
\mathbb{E}\left(\epsilon_{2}(t) A_{1}^{*} \sum_{i=0}^{\infty} \mathbf{A}^{i} U(t-i)\right) & \vdots & \mathbb{E}\left(\epsilon_{2}(t) A_{1}^{*} \sum_{i=0}^{\infty} \mathbf{A}^{i} U\left(t-L_{j}+1-i\right)\right) \\
\vdots & \ddots & \vdots \\
\mathbb{E}\left(\epsilon_{2}\left(t-L_{j}+1\right) A_{1}^{*} \sum_{i=0}^{\infty} \mathbf{A}^{i} U(t-i)\right) & \vdots & \mathbb{E}\left(\epsilon_{2}\left(t-L_{j}+1\right) A_{1}^{*} \sum_{i=0}^{\infty} \mathbf{A}^{i} U\left(t-L_{j}+1-i\right)\right)
\end{array}\right] }
\end{aligned}
$$

However, $\mathbb{E}\left(\epsilon_{2}(t-k) A_{1}^{*} \sum_{i=0}^{\infty} \mathbf{A}^{i} U(t-i)\right) \neq 0 \Leftrightarrow i=k, \forall k$. Then $\forall k \in\{0,1,2, \ldots\}$, 


$$
\begin{aligned}
& U^{\top}(t-k)=[\boldsymbol{\epsilon}(t), 0, \ldots, 0]=\left[\epsilon_{1}(t-k), \epsilon_{2}(t-k), \ldots, \epsilon_{D}(t-k), 0, \ldots, 0\right]_{(1 \times p D)}: \\
& \mathbb{E}\left(\epsilon_{2}(t-k) A_{1}^{*} \sum_{i=0}^{\infty} \mathbf{A}^{i} U(t-i)\right)=\mathbb{E}\left(\epsilon_{2}(t-k) A_{1}^{*} \mathbf{A}^{k} U(t-k)\right)= \\
& =\mathbb{E}\left\{A_{1}^{*} \mathbf{A}^{k}\left[\begin{array}{c}
\epsilon_{1}(t-k) \epsilon_{2}(t-k) \\
\epsilon_{2}(t-k) \epsilon_{2}(t-k) \\
\vdots \\
\epsilon_{D}(t-k) \epsilon_{2}(t-k) \\
0 \\
\vdots \\
0
\end{array}\right]\right\}=A_{1}^{*} \mathbf{A}^{k} \mathbb{E}\left\{\left[\begin{array}{c}
\epsilon_{1}(t-k) \epsilon_{2}(t-k) \\
\epsilon_{2}(t-k) \epsilon_{2}(t-k) \\
\vdots \\
\epsilon_{D}(t-k) \epsilon_{2}(t-k)
\end{array}\right]\right\}= \\
& =A_{1}^{*} \mathbf{A}^{k}\left[\begin{array}{c}
0 \\
\sigma\left(\frac{t-k}{T}\right) \\
\vdots \\
0
\end{array}\right]=\left[a_{1 \bullet}^{(1)}(u), \ldots, a_{1 \cdot}^{(p)}(u)\right]\left[\begin{array}{ccc}
\bar{a}_{11}^{(k)}(u) & \ldots & \bar{a}_{1, p D}^{(k)}(u) \\
\vdots & \ddots & \vdots \\
\bar{a}_{p D, 1}^{(k)}(u) & \ldots & \bar{a}_{p D, p D}^{(k)}(u)
\end{array}\right]\left[\begin{array}{c}
0 \\
\sigma\left(\frac{t-k}{T}\right) \\
\vdots \\
0
\end{array}\right] \\
& =\left[a_{1}^{(1)}(u), \ldots, a_{1}^{(p)}(u)\right]\left[\begin{array}{c}
\bar{a}_{1,2}^{(k)}(u) \\
\vdots \\
\bar{a}_{p D, 2}^{(k)}(u)
\end{array}\right] \sigma\left(\frac{t-k}{T}\right)=\left(A_{1}^{*}\right)\left(\bar{A}_{\cdot 2}^{(k)}\right) \sigma\left(\frac{t-k}{T}\right) .
\end{aligned}
$$

Finally we have:

$$
\begin{aligned}
& \mathbb{E}\left\{\widetilde{W}_{j, t}\left(\epsilon_{2}(t)\right)\left[\sum_{i=1}^{D} \mathcal{W}_{j, t}\left(\mathcal{F}_{1, i}(t)\right)\right]\right\}= \\
& =\tilde{\mathbf{h}}_{\mathbf{j}}^{\top}\left[\begin{array}{ccc}
\mathbb{E}\left(\epsilon_{2}(t) A_{1}^{*} \sum_{i=0}^{\infty} \mathbf{A}^{i} U_{t-i}\right) & \ldots & \mathbb{E}\left(\epsilon_{2}(t) A_{1}^{*} \sum_{i=0}^{\infty} \mathbf{A}^{i} U_{t-L_{j}+1-i}\right) \\
\vdots & \ddots & \vdots \\
\mathbb{E}\left(\epsilon_{2}\left(t-L_{j}+1\right) A_{1}^{*} \sum_{i=0}^{\infty} \mathbf{A}^{i} U_{t-i}\right) & \ldots & \mathbb{E}\left(\epsilon_{2}\left(t-L_{j}+1\right) A_{1}^{*} \sum_{i=0}^{\infty} \mathbf{A}^{i} U_{t-L_{j}+1-i}\right)
\end{array}\right] \tilde{\mathbf{h}}_{\mathbf{j}}= \\
& =\tilde{\mathbf{h}}_{\mathbf{j}}^{\top}\left[\begin{array}{ccc}
\left(A_{1}^{*}\right)\left(\bar{A}_{\cdot 2}^{(0)}\right) \sigma\left(\frac{t-k}{T}\right) & \ldots & \left(A_{1}^{*}\right)\left(\bar{A}_{\cdot 2}^{(0)}\right) \sigma\left(\frac{t-k}{T}\right) \\
\vdots & \ddots & \vdots \\
\left(A_{1}^{*}\right)\left(\bar{A}_{\cdot 2}^{\left(L_{j}-1\right)}\right) \sigma\left(\frac{t-k}{T}\right) & \ldots & \left(A_{1}^{*}\right)\left(\bar{A}_{\cdot 2}^{\left(L_{j}-1\right)}\right) \sigma\left(\frac{t-k}{T}\right)
\end{array}\right] \tilde{\mathbf{h}}_{\mathbf{j}}= \\
& =\tilde{\mathbf{h}}_{\mathbf{j}}^{\top}\left[\begin{array}{ccc}
\left(A_{1}^{*}\right)\left(\bar{A}_{\cdot 2}^{(0)}\right) & \ldots & \left(A_{1}^{*}\right)\left(\bar{A}_{\cdot 2}^{(0)}\right) \\
\vdots & \ddots & \vdots \\
\left(A_{1}^{*}\right)\left(\bar{A}_{\cdot 2}^{\left(L_{j}-1\right)}\right) & \ldots & \left(A_{1}^{*}\right)\left(\bar{A}_{\cdot 2}^{\left(L_{j}-1\right)}\right)
\end{array}\right] \tilde{\mathbf{h}}_{\mathbf{j}} \cdot \sigma\left(\frac{t-k}{T}\right)= \\
& =\tilde{\mathbf{h}}_{\mathbf{j}}^{\top}\left[\left(\begin{array}{cccc}
A_{1}^{*} & 0 & \ldots & 0 \\
0 & A_{1}^{*} & \ldots & 0 \\
\vdots & \vdots & \ddots & \vdots \\
0 & 0 & \ldots & A_{1}^{*}
\end{array}\right)\left(\begin{array}{c}
\left(\bar{A}_{\cdot 2}^{(0)}\right) \\
\vdots \\
\left(\bar{A}_{\cdot 2}^{\left(L_{j}-1\right)}\right)
\end{array}\right) \cdot\left({\underset{\sim}{1}}^{\top}\right)_{\left(1 \times L_{j}\right)}\right] \tilde{\mathbf{h}}_{\mathbf{j}} \cdot \sigma\left(\frac{t-k}{T}\right)=
\end{aligned}
$$




$$
\begin{aligned}
& =\tilde{\mathbf{h}}_{\mathbf{j}}^{\top}\left[(\underset{\sim}{1})_{\left(L_{j} \times 1\right)} \cdot\left(A_{1}^{*}\right)_{(1 \times p D)} \cdot\left(\begin{array}{ccc}
\left(\sim_{\sim}^{\top}\right)_{(1 \times p D)} & \cdots & 0 \\
\vdots & \ddots & \vdots \\
0 & \cdots & \left(\sim_{\sim}^{1}\right)_{(1 \times p D)}
\end{array}\right)_{\left(p D \times L_{j} p D\right)}\right. \\
& \left(\begin{array}{c}
\left(\bar{A}_{\cdot 2}^{(0)}\right)_{(p D \times 1)} \\
\vdots \\
\left(\bar{A}_{\cdot 2}^{\left(L_{j}-1\right)}\right)_{(p D \times 1)}
\end{array}\right)_{L_{j} p D \times 1} \cdot\left({\underset{\sim}{1}}^{\top}\right)_{\left(1 \times L_{j}\right)} \tilde{\mathbf{h}}_{\mathbf{j}} \cdot \sigma\left(\frac{t-k}{T}\right)= \\
& =\tilde{\mathbf{h}}_{\mathbf{j}}^{\top}\left[(\underset{\sim}{1}) \cdot\left(A_{1}^{*}\right) \cdot \operatorname{diag}\left({\underset{\sim}{1}}^{\top}\right)\left(\begin{array}{c}
\left(\bar{A}_{\cdot 2}^{(0)}\right) \\
\vdots \\
\left(\bar{A}_{\cdot 2}^{\left(L_{j}-1\right)}\right)
\end{array}\right) \cdot\left({\underset{\sim}{1}}^{\top}\right)\right] \tilde{\mathbf{h}}_{\mathbf{j}} \cdot \sigma\left(\frac{t-k}{T}\right)
\end{aligned}
$$

where $\operatorname{diag}\left({\underset{\sim}{1}}^{\top}\right)$ is a block diagonal matrix with blocks of matrices $\left(\sim_{\sim}^{(1}\right)_{1 \times p D}=\left(1_{p D \text { times }} 1\right)$ on its diagonal. By analogy, equation VII results in:

$$
\begin{aligned}
& \mathbb{E}\left\{\widetilde{W}_{j, t}\left(\epsilon_{1}(t)\right)\left[\sum_{i=1}^{D} \mathcal{W}_{j, t}\left(\mathcal{F}_{2, i}(t)\right)\right]\right\}= \\
= & \tilde{\mathbf{h}}_{\mathbf{j}}^{\top}\left[(\underset{\sim}{1}) \cdot\left(A_{2}^{*}\right) \cdot \operatorname{diag}\left({\underset{\sim}{1}}^{\top}\right)\left(\begin{array}{c}
\left(\bar{A}_{\cdot 1}^{(0)}\right) \\
\vdots \\
\left(\bar{A}_{\cdot 1}^{\left(L_{j}-1\right)}\right)
\end{array}\right) \cdot\left(\underset{\sim}{\left.1^{\top}\right)}\right] \tilde{\mathbf{h}}_{\mathbf{j}} \cdot \sigma\left(\frac{t-k}{T}\right)\right.
\end{aligned}
$$

The result of the sum between VII and VIII is therefore:

$$
\begin{aligned}
& \tilde{\mathbf{h}}_{\mathbf{j}}^{\top}\left[\underset{\sim}{1} \cdot A_{1}^{*} \cdot \operatorname{diag}\left(\sim_{\sim}^{\top}\right)\left(\begin{array}{c}
\left(\bar{A}_{\cdot 2}^{(0)}\right) \\
\vdots \\
\left(\bar{A}_{\cdot 2}^{\left(L_{j}-1\right)}\right)
\end{array}\right) \stackrel{\sim}{\sim}^{\top}\right] \tilde{\mathbf{h}}_{\mathbf{j}} \cdot \sigma\left(\frac{t-k}{T}\right)+ \\
& +\quad \tilde{\mathbf{h}}_{\mathbf{j}}^{\top}\left[\underset{\sim}{1} \cdot A_{2}^{*} \cdot \operatorname{diag}\left({\underset{\sim}{1}}^{\top}\right)\left(\begin{array}{c}
\left(\bar{A}_{\cdot 1}^{(0)}\right) \\
\vdots \\
\left(\bar{A}_{\cdot 1}^{\left(L_{j}-1\right)}\right)
\end{array}\right) \sim_{\sim}^{\top}\right] \tilde{\mathbf{h}}_{\mathbf{j}} \cdot \sigma\left(\frac{t-k}{T}\right)=
\end{aligned}
$$

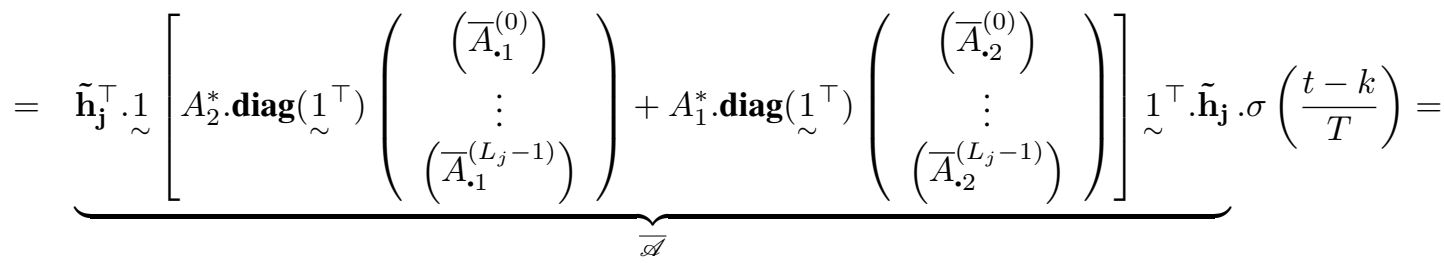

$$
\begin{aligned}
& =\overline{\mathscr{A}} \cdot \sigma\left(\frac{t-k}{T}\right)
\end{aligned}
$$

- Calculating IX: 
This is the term that has the relations of interest between $X_{t, T}^{(1)}$ and $X_{t, T}^{(2)}$. However, it also has all other relations between $X_{t, T}^{(1)}$ and $X_{t, T}^{(2)}$ and the other components of $\mathbf{X}_{t, T}$. We must then explicit all of these relations and isolate only the interesting relations that will compose the ppDWC.

$$
\begin{aligned}
& \mathbb{E}\left\{\mathcal{W}_{j, t}\left(\mathcal{F}_{1,1}(t)\right)\left[\sum_{i=1}^{D} \mathcal{W}_{j, t}\left(\mathcal{F}_{2, i}(t)\right)\right]+\cdots+\mathcal{W}_{j, t}\left(\mathcal{F}_{1, D}(t)\right)\left[\sum_{i=1}^{D} \mathcal{W}_{j, t}\left(\mathcal{F}_{2, i}(t)\right)\right]\right\}= \\
= & \sum_{i=1}^{D} \mathbb{E}\left\{\mathcal{W}_{j, t}\left(\mathcal{F}_{1, i}(t)\right)\left[\sum_{i^{\prime}=1}^{D} \mathcal{W}_{j, t}\left(\mathcal{F}_{2, i^{\prime}}(t)\right)\right]\right\}=\sum_{i=1}^{D} \sum_{i^{\prime}=1}^{D} \mathbb{E}\left\{\mathcal{W}_{j, t}\left(\mathcal{F}_{1, i}(t)\right) \mathcal{W}_{j, t}\left(\mathcal{F}_{2, i^{\prime}}(t)\right)\right\}= \\
= & \sum_{i=1}^{D} \sum_{i^{\prime}=1}^{D} \mathbb{E}\left\{\left[\sum_{l=0}^{L_{j}-1} \widetilde{h}_{j, l} \sum_{m=1}^{p} a_{1, i}^{(m)}(u) X_{t-l-m, T}^{(i)}\right]\left[\sum_{l^{\prime}=0}^{L_{j}-1} \widetilde{h}_{j, l^{\prime}} \sum_{m^{\prime}=1}^{p} a_{2, i^{\prime}}^{\left(m^{\prime}\right)}(u) X_{t-l^{\prime}-m^{\prime}, T}^{\left(i^{\prime}\right)}\right]\right\}= \\
= & \sum_{i=1}^{D} \sum_{i^{\prime}=1}^{D} \mathbb{E}\left\{\left[\sum_{l=0}^{L_{j}-1} \sum_{m=1}^{p} \widetilde{h}_{j, l} a_{1, i}^{(m)}(u) X_{t-l-m, T}^{(i)}\right]\left[\sum_{l^{\prime}=0}^{L_{j}-1} \sum_{m^{\prime}=1}^{p} \widetilde{h}_{j, l^{\prime}} a_{2, i^{\prime}}^{\left(m^{\prime}\right)}(u) X_{t-l^{\prime}-m^{\prime}, T}^{\left(i^{\prime}\right)}\right]\right\}= \\
= & \sum_{i=1}^{D} \sum_{i^{\prime}=1}^{D} \sum_{l=0}^{L_{j}-1} \sum_{l^{\prime}=0}^{L_{j}-1} \sum_{m=1}^{p} \sum_{m^{\prime}=1}^{p} \widetilde{h}_{j, l} \widetilde{h}_{j, l^{\prime}} a_{1, i}^{(m)}(u) a_{2, i^{\prime}}^{\left(m^{\prime}\right)}(u) \mathbb{E}\left\{X_{t-l-m, T}^{(i)} X_{t-l^{\prime}-m^{\prime}, T}^{\left.(i)^{\prime}\right)}\right\}= \\
= & \sum_{i=1}^{D} \sum_{i^{\prime}=1}^{D} \sum_{l=0}^{L_{j}-1} \sum_{l^{\prime}=0}^{L_{j}-1} \sum_{m=1}^{p} \sum_{m^{\prime}=1}^{p} \widetilde{h}_{j, l} \widetilde{h}_{j, l^{\prime}} a_{1, i}^{(m)}(u) a_{2, i^{\prime}}^{\left(m^{\prime}\right)}(u) \gamma_{X_{i}, X_{i^{\prime}}}\left(t-l-m, t-l^{\prime}-m^{\prime}\right) \quad(\mathrm{B} .8)
\end{aligned}
$$

Now, for the quantity presented in (B.8), with respect to the indices $i, i^{\prime}, l, l^{\prime}, m, m^{\prime}$ we have the following situations. A and B in the third and fourth columns are set according to Figure B.1. The arrow ' $\rightleftharpoons$ ' represents bilateral relation and ' $\rightarrow$ ' represents unilateral relation.

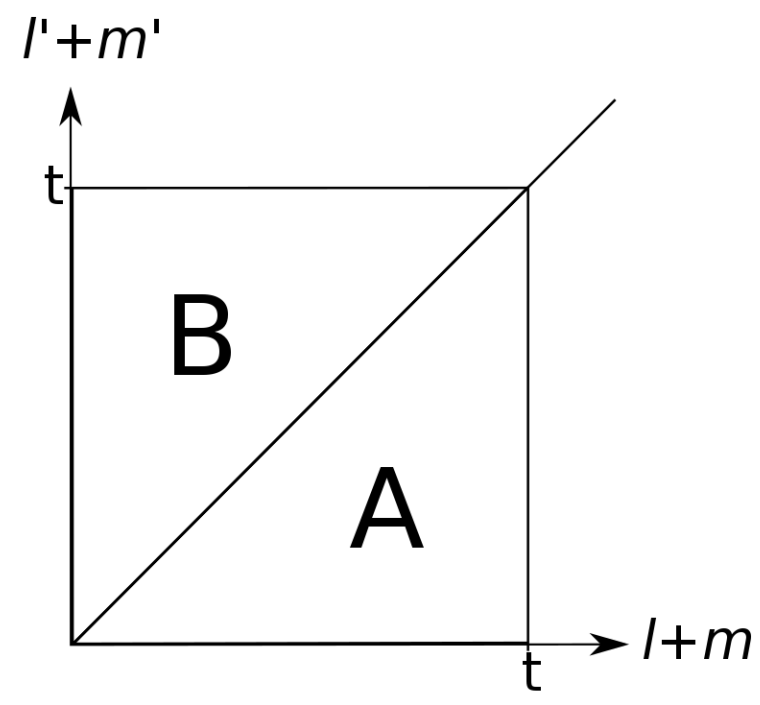

Figure B.1. $(l+m) \times\left(l^{\prime}+m^{\prime}\right)$ : regions $A$ and $B$ represent $l+m>l^{\prime}+m^{\prime}$ and $l+m<l^{\prime}+m^{\prime}$ respectively. 


\begin{tabular}{|l|l|c|c|}
\hline & Influences & $(l+m) \times\left(l^{\prime}+m^{\prime}\right) \in A$ & $(l+m) \times\left(l^{\prime}+m^{\prime}\right) \in B$ \\
\hline i. & $X_{1} \leftrightarrow X_{1}$ & \multicolumn{2}{|c|}{$i=1, i^{\prime}=1$} \\
ii. & $X_{2} \leftrightarrow X_{2}$ & \multicolumn{2}{|c|}{$i=2, i^{\prime}=2$} \\
iii. & $X_{1} \rightleftharpoons X_{2}$ & \multicolumn{2}{|c|}{$i=1, i^{\prime}=2,(l-m)=\left(l^{\prime}-m^{\prime}\right)$} \\
iv. & $X_{1} \rightarrow X_{2}$ & $i=1, i^{\prime}=2$ & $i=2, i^{\prime}=1$ \\
v. & $X_{2} \rightarrow X_{1}$ & $i=2, i^{\prime}=1$ & $i=1, i^{\prime}=2$ \\
vi. & $X_{1} \rightarrow\left\{X_{3}, \ldots, X_{D}\right\}$ & $i=1, i^{\prime} \in\{3, \ldots, D\}$ & $i \in\{3, \ldots, D\}, i^{\prime}=1$ \\
vii. & $X_{2} \rightarrow\left\{X_{3}, \ldots, X_{D}\right\}$ & $i=2, i^{\prime} \in\{3, \ldots, D\}$ & $i \in\{3, \ldots, D\}, i^{\prime}=2$ \\
viii. & $\left\{X_{3}, \ldots, X_{D}\right\} \rightarrow X_{1}$ & $i \in\{3, \ldots, D\}, i^{\prime}=1$ & $i=1, i^{\prime} \in\{3, \ldots, D\}$ \\
ix. & $\left\{X_{3}, \ldots, X_{D}\right\} \rightarrow X_{2}$ & $i \in\{3, \ldots, D\}, i^{\prime}=2$ & $i=2, i^{\prime} \in\{3, \ldots, D\}$ \\
x. & All interactions within $\left\{X_{3}, \ldots, X_{D}\right\}$ & \multicolumn{2}{|c|}{$\left(i, i^{\prime}\right) \in\{3, \ldots, D\} \times\{3, \ldots, D\}$} \\
\hline
\end{tabular}

Table B.1. Categories of relations between components of $\mathbf{X}_{t, T}$. Arrows ' $\rightleftharpoons$ ' represent bilateral relation while ' $\rightarrow$ ' represent unilateral relation. $A$ and $B$ are defined in Figure B.1.

The relations $i$ and $i i$ tell us about the relation between $X_{1}$ and $X_{2}$ with their own past while relation iii measures instantaneous relation between $X_{1}$ and $X_{2}$. Relations $i v$ and $v$ measure the impact of $X_{1}$ in $X_{2}$ and the impact of $X_{2, t, T}$ in $X_{1, t, T}$, respectively, without the influence of the other components $\left\{X_{3}, \ldots, X_{D}\right\}$, that are isolated in relations $v i$ to $i x$. Thus we can write equation (B.8) as:

$$
\begin{aligned}
& p p F_{X_{1}, X_{2}, t}\left(\tau_{j}\right)+p p B_{X_{1}, X_{2}, t}\left(\tau_{j}\right)+ \\
+ & R_{0}\left(X_{1} \rightleftharpoons X_{2}, \tau_{j}, t\right)+R\left(X_{1} \leftrightarrow X_{1}, \tau_{j}, t\right)+R\left(X_{2} \leftrightarrow X_{2}, \tau_{j}, t\right)+ \\
+ & R\left(X_{1} \leftrightarrow\left\{X_{3}, \ldots, X_{D}\right\}, \tau_{j}, t\right)+R\left(X_{2} \leftrightarrow\left\{X_{3}, \ldots, X_{D}\right\}, \tau_{j}, t\right)+ \\
+ & R\left(\left\{X_{3}, \ldots, X_{D}\right\} \leftrightarrow\left\{X_{3}, \ldots, X_{D}\right\}, \tau_{j}, t\right)
\end{aligned}
$$

with

$$
\begin{aligned}
& p p F_{X_{1}, X_{2}, t}\left(\tau_{j}\right)=\sum_{A} \widetilde{h}_{j, l} \widetilde{h}_{j, l^{\prime}} a_{1,1}^{(m)}(u) a_{2,2}^{\left(m^{\prime}\right)}(u) \gamma_{X_{1}, X_{2}}\left(t-l-m, t-l^{\prime}-m^{\prime}\right)+ \\
& \quad+\sum_{B} \widetilde{h}_{j, l} \widetilde{h}_{j, l^{\prime}} a_{1,2}^{(m)}(u) a_{2,1}^{\left(m^{\prime}\right)}(u) \gamma_{X_{2}, X_{1}}\left(t-l-m, t-l^{\prime}-m^{\prime}\right) \\
& p p B_{X_{1}, X_{2}, t}\left(\tau_{j}\right)=\sum_{B} \widetilde{h}_{j, l} \widetilde{h}_{j, l^{\prime}} a_{1,1}^{(m)}(u) a_{2,2}^{\left(m^{\prime}\right)}(u) \gamma_{X_{1}, X_{2}}\left(t-l-m, t-l^{\prime}-m^{\prime}\right)+ \\
& \quad+\sum_{A} \widetilde{h}_{j, l} \widetilde{l}_{j, l^{\prime}} a_{1,2}^{(m)}(u) a_{2,1}^{\left(m^{\prime}\right)}(u) \gamma_{X_{2}, X_{1}}\left(t-l-m, t-l^{\prime}-m^{\prime}\right) \\
& R\left(X_{1} \leftrightarrow X_{1}, \tau_{j}, t\right)=\sum_{l=0}^{L_{j}-1} \sum_{l^{\prime}=0}^{L_{j}-1} \sum_{m=1}^{p} \sum_{m^{\prime}=1}^{p} \widetilde{h}_{j, l} \widetilde{h}_{j, l^{\prime}} a_{1,1}^{(m)}(u) a_{2,1}^{\left(m^{\prime}\right)}(u) \gamma_{X_{1}, X_{1}}\left(t-l-m, t-l^{\prime}-m^{\prime}\right) \\
& R\left(X_{2} \leftrightarrow X_{2}, \tau_{j}, t\right)=\sum_{l=0}^{L_{j}-1} \sum_{l^{\prime}=0}^{L_{j}-1} \sum_{m=1}^{p} \sum_{m^{\prime}=1}^{p} \widetilde{h}_{j, l} \widetilde{h}_{j, l^{\prime}} a_{1,2}^{(m)}(u) a_{2,2}^{\left(m^{\prime}\right)}(u) \gamma_{X_{2}, X_{2}}\left(t-l-m, t-l^{\prime}-m^{\prime}\right) \\
& R_{0}\left(X_{1} \rightleftharpoons X_{2}, \tau_{j}, t\right)=\sum_{l-m=l^{\prime}-m^{\prime}} \widetilde{h}_{j, l} \widetilde{h}_{j, l^{\prime}} a_{1,2}^{(m)}(u) a_{2,1}^{\left(m^{\prime}\right)}(u) \gamma_{X_{2}, X_{1}}\left(t-l-m, t-l^{\prime}-m^{\prime}\right)+ \\
& \quad+\sum_{l-m=l^{\prime}-m^{\prime}} \widetilde{h}_{j, l} \widetilde{h}_{j, l^{\prime}}^{(m)}(u) a_{2,2}^{\left(m^{\prime}\right)}(u) \gamma_{X_{1}, X_{2}}\left(t-l-m, t-l^{\prime}-m^{\prime}\right)
\end{aligned}
$$




$$
\begin{aligned}
R( & X_{1} \\
\leftrightarrow & \left.\left\{X_{3}, \ldots, X_{D}\right\}, \tau_{j}, t\right)=R\left(X_{1} \rightarrow\left\{X_{3}, \ldots, X_{D}\right\}\right)+R\left(\left\{X_{3}, \ldots, X_{D}\right\} \rightarrow X_{1}\right)= \\
= & \sum_{i^{\prime}=3}^{D} \sum_{l=0}^{L_{j}-1} \sum_{l^{\prime}=0}^{L_{j}-1} \sum_{m=1}^{p} \sum_{m^{\prime}=1}^{p} \widetilde{h}_{j, l} \widetilde{h}_{j, l^{\prime}} a_{1,1}^{(m)}(u) a_{2, i^{\prime}}^{\left(m^{\prime}\right)}(u) \gamma_{X_{1}, X_{i^{\prime}}}\left(t-l-m, t-l^{\prime}-m^{\prime}\right)+ \\
& +\sum_{i=3}^{D} \sum_{l=0}^{L_{j}-1} \sum_{l^{\prime}=0}^{L_{j}-1} \sum_{m=1}^{p} \sum_{m^{\prime}=1}^{p} \widetilde{h}_{j, l} \widetilde{h}_{j, l^{\prime}} a_{1, i}^{(m)}(u) a_{2,1}^{\left(m^{\prime}\right)}(u) \gamma_{X_{i}, X_{1}}\left(t-l-m, t-l^{\prime}-m^{\prime}\right) \\
R\left(X_{2}\right. & \left.\leftrightarrow\left\{X_{3}, \ldots, X_{D}\right\}, \tau_{j}, t\right)=R\left(X_{2} \rightarrow\left\{X_{3}, \ldots, X_{D}\right\}\right)+R\left(\left\{X_{3}, \ldots, X_{D}\right\} \rightarrow X_{2}\right) \\
= & \sum_{i=3}^{D} \sum_{l=0}^{L_{j}-1} \sum_{l^{\prime}=0}^{L_{j}-1} \sum_{m=1}^{p} \sum_{m^{\prime}=1}^{p} \widetilde{h}_{j, l} \widetilde{h}_{j, l^{\prime}} a_{1, i}^{(m)}(u) a_{2,2}^{\left(m^{\prime}\right)}(u) \gamma_{X_{i}, X_{2}}\left(t-l-m, t-l^{\prime}-m^{\prime}\right)+ \\
& +\sum_{i^{\prime}=3}^{D} \sum_{l=0}^{L_{j}-1} \sum_{l^{\prime}=0}^{L_{j}-1} \sum_{m=1}^{p} \sum_{m^{\prime}=1}^{p} \widetilde{h}_{j, l} \widetilde{h}_{j, l^{\prime}} a_{1,2}^{(m)}(u) a_{1, i^{\prime}}^{\left(m^{\prime}\right)}(u) \gamma_{X_{2}, X_{i^{\prime}}}\left(t-l-m, t-l^{\prime}-m^{\prime}\right) \\
R\left(\left\{X_{3}, \ldots, X_{D}\right\} \leftrightarrow\left\{X_{3}, \ldots, X_{D}\right\}, \tau_{j}, t\right)= & \\
= & \sum_{i=3}^{D} \sum_{i^{\prime}=3}^{D} \sum_{l=0}^{L_{j}-1} \sum_{l^{\prime}=0}^{L_{j}-1} \sum_{m=1}^{p} \sum_{m^{\prime}=1}^{p} \widetilde{h}_{j, l} \widetilde{h}_{j, l^{\prime}} a_{1, i}^{(m)}(u) a_{2, i^{\prime}}^{\left(m^{\prime}\right)}(u) \gamma_{X_{i}, X_{i^{\prime}}}\left(t-l-m, t-l^{\prime}-m^{\prime}\right)
\end{aligned}
$$

where $A=\left\{\left(l, m, l^{\prime} m^{\prime}\right) \mid l-m>l^{\prime}-m^{\prime}, m, m^{\prime} \in\{1, \ldots, p\}, l, l^{\prime} \in\left\{0, \ldots, L_{j}-1\right\}\right\}$ and $B=\left\{\left(l, m, l^{\prime} m^{\prime}\right) \mid l-m<l^{\prime}-m^{\prime}, m, m^{\prime} \in\{1, \ldots, p\}, l, l^{\prime} \in\left\{0, \ldots, L_{j}-1\right\}\right\}$ as represented graphically in Figure B.1 . Notice that we use ' $\rightarrow$ ' to represent directed (from-to) relation, ' $\rightleftharpoons$ ' represents instantaneous relation and ' $\leftrightarrow$ ' for all possible combinations of relationship between the related components.

Then rejoining $\mathbf{I}$ to $\mathbf{I X}$ again into $\nu\left(X^{(1)}, X^{(2)}, \tau_{j}\right)$ we have:

$$
\begin{aligned}
\nu\left(X^{(1)}, X^{(2)}, \tau_{j}\right)= & \operatorname{Cov}\left(\widetilde{W}_{j, t}^{(1)}, \widetilde{W}_{j, t}^{(2)}\right)=\widetilde{W}_{j, t}\left(\mu_{1}(u)\right) \widetilde{W}_{j, t}\left(\mu_{2}(u)\right)+ \\
& +p p F_{X_{1}, X_{2}, t}\left(\tau_{j}\right)+p p B_{X_{1}, X_{2}, t}\left(\tau_{j}\right)+ \\
& +R\left(X_{1} \rightleftharpoons X_{1}, \tau_{j}, t\right)+R\left(X_{2} \rightleftharpoons X_{2}, \tau_{j}, t\right)+R\left(X_{1} \rightleftharpoons X_{2}, \tau_{j}, t\right)+ \\
& +R\left(X_{1} \leftrightarrow\left\{X_{3}, \ldots, X_{D}\right\}, \tau_{j}, t\right)+R\left(X_{2} \leftrightarrow\left\{X_{3}, \ldots, X_{D}\right\}, \tau_{j}, t\right)+ \\
& +R\left(\left\{X_{3}, \ldots, X_{D}\right\} \leftrightarrow\left\{X_{3}, \ldots, X_{D}\right\}, \tau_{j}, t\right)+ \\
& +\overline{\mathscr{A}} \cdot \sigma\left(\frac{t-k}{T}\right),
\end{aligned}
$$

with $\overline{\mathscr{A}}$ as in (B.7), $R($.$) as in (B.9), p p F($.$) and p p B($.$) as in (B.10) and (B.11), respectively.$ 


\section{Bibliography}

Arnold, M., Miltner, W. H. R., Witte, H., Bauer, R., and Braun, C. (1998). Adaptive ar modeling of nonstationary time series by means of kalman filtering. IEEE Trans. Biomed. Eng., 45:553-562. 1

Baccalá, L. A. and Sameshima, K. (2001). Partial directed coherence: A new concept in neural structure determination. Biological Cybernetics, 84:463-474. 1, 3, 5, 11, 13

Bloomfield, P. (2000). Fourier Analysis of Time Series: An Introduction. Wiley, New York, 2 edition. 11

Brillinger, D. R. (2001). Time Series: Data Analysis and Theory. New York: Holt, Rinehart and Winston, New York, expanded edition. 7, 10

Dahlhaus, R. (1996). Asymptotic statistical inference for nonstationary processes with evolutionary spectra. In: Robinson P.M. and Rosenblatt M. (eds.). Athens Conference on Applied Probability and Time Series Analysis, 2:145-159. 4, 16, 17, 36, 37, 80, 82

Dahlhaus, R. and Polonik, W. (2006). Asymptotic statistical inference for nonstationary processes with evolutionary spectra. The Annals of Statistics, 34(6):2790-2824. 18

Dahlhaus, R. and Polonik, W. (2009). Empirical spectral processes for locally stationary time series. Bernoulli, 15(1):1-39. 19

Daubechies, I. (1988). Orthonormal bases of compactly supported wavelets. Comm. Pure Applied Mathematics, 41:909-996. 20

Daubechies, I. (1992). Ten lectures on wavelets. SIAM. 20

Fourier, J. B. J. (1822). Théorie Analytique de la Chaleur. Éditions Jacques Gabay, Paris, France, reprint, 1988 edition. 5

Ginter, J., Blinowska, K. J., Kaminski, M., Durka, P. J., Pfurtscheller, G., and Neuper, C. (2005). Propagation of eeg activity in the beta and gamma band during movement imagery in humans. Methods inf Med, 44:106-113. 79

Granger, C. W. J. (1969). Investigating causal relations by econometric models and cross-spectral methods. Econometrica, 37. 1

Grinsted, A., Moore, J. C., and Jevrejeva, S. (2004). Application of the cross wavelet transform and wavelet coherence to geophysical time series. Nonlinear Processes in Geophysics, 11:561-566. 1, $3,33,60$

Härdle, W., Kerkyacharian, G., Picard, D., and Tsybakov, A. (1997). Wavelets, Approximation and Statistical Applications. Springer, Paris, France. 2, 22, 23

Jeon, S., Nicolis, O., and Vidakovic, B. (2014). Mammogram diagnostics via 2-d complex waveletbased self-similarity measures. São Paulo Journal of Mathematical Sciences, 8(2):265-284. 83 
Lopes, K. S. M. (2014). Partial directed coherence applications on eeg data. Advances and Applications in Statistics, 42:65-82. 1, 13, 57

Lütkepohl, H. (2007). New Introduction to Multiple Time Series Analysis. Springer Berlin Heidelberg, New York. 38, 117

Martin, W. and Flandrin, P. (1985). Wigner-ville spectral analysis of nonstationary processes. ASSP$33,6: 1461-1470.15$

Matz, G., Hlawatsch, F., and Kozek, W. (1997). Generalized evolutionary spectral analysis and the weyl spectrum of nonstationary random processes. IEEE Transactions on Signal Processing, 45(6):1520-1534. 16

Milde, T., Schwab, K., Walther, M., Eiselt, M., Schelenz, C., Voss, A., and Witte, H. (2011). Timevariant partial directed coherence in analysis of the cardiovascular system. a methodological study. Physiol. Meas., 32:1787-1805. 1

Morettin, P. A. (2014). Waves and Wavelets. University of São Paulo Press, São Paulo, 2nd edition. $7,8,16,19,23,25,26,27,38,80$

Nason, G. P., von Sachs, R., and Kroisandt, G. (2000). Wavelet processes and adaptive estimation of the evolutionary wavelet spectrum. J. R. Statist. Soc., 62:271-292. 19, 80, 83

Neumann, M. H. and von Sachs, R. (1997). Wavelet thresholding in anisotropic function classes and application to adaptative estimation of evolutionary spectra. The Annals of Statistics, 25:38-76. 19

Percival, D. B. and Walden, A. T. (1993). Spectral Analysis for Physical Applications. Cambridge University Press, New York. 5

Percival, D. B. and Walden, A. T. (2000). Wavelet Methods for Time Series Analysis. Cambridge University Press, New York. 3, 5, 24, 28, 29, 33

Pester, B., Ligges, C., Leistritz, L., Witte, H., and K.Schiecke (2015). Advanced insights into functional brain connectivity by combining tensor decomposition and partial directed coherence. PLoS ONE, 10(6). 1

Priestley, M. B. (1965). Evolutionary spectra and non-stationary processes. J. R. Statist. Soc., 27(2):204-237. 4, 16, 36

Priestley, M. B. (1981). Spectral Analysis and Time Series. London: Academic Press, London. 1, 5, 16,17

Priestley, M. B. (1996). Wavelets and time-dependent spectral analysis. Journal of Time Series Analysis, 17(1):85-103. 17

Priestley, M. B. and Tong, H. (1973). On the analysis of bivariate non-stationary processes. Journal of the Royal Statistical Society, 35(2):153-166. 17

Remenyi, N., Nicolis, O., Nason, G., and Vidakovic, B. (2014). Image denoising with $2 \mathrm{~d}$ scalemixing complex wavelet transforms. IEEE Transactions on Image Processing, 23(12):5165-5174. 83

Saito, Y. and Harashima, H. (1981). Tracking of information within multichannel eeg record - causal analysis in eeg. Recent Advantages in EEG and EMG Data Processing, pages 133-146. 1, 11 
Sato, J. R., Junior, E. A., Takahashi, D. Y., Felix, M. M., Brammer, J. M., and Morettin, P. A. (2006). A method to produce evolving functional connectivity maps during the course of an fmri experiment using wavelet-based time-varying granger causality. NeuroImage, 31:187-196. 57

Sato, J. R., Morettin, P. A., Arantes, P. R., and Junior, E. A. (2007). Wavelet based time-varying vector autoregressive modelling. Computational Statistics \& Data Analysis, 51:5847-5866. 1, 21

Subba Rao, T. and Gabr, M. M. (1984). An Introduction to Bispectral Analysis and Bilinear Time Series Models, volume Lecture Notes in Statistics, V.24. Springer-Verlag, New York. 16

Takahashi, D. Y., Baccalá, L. A., and Sameshima, K. (2007). On partial directed coherence factor, granger causality and information theory. 10th Tamagawa-Riken Brain Dynamics Brain Forum DBF07. 1, 11

Tong, H. (1990). Nonlinear Time Series: A Dynamical System Approach. Oxford University Press, New York. 16

Torrence, C. and Compo, G. P. (1998). A practical guide to wavelet analysis. Bulletin of the American Meteorological Society, 79:61-78. 1, 5, 33 


\section{Index}

Coherence, 9

Parital, 11

Partial Directed, 11

Covariance

Covariance Function, 6

cross-covariance function, 8

Cross Spectra Matrix, 9

Cross Spectrum, 8

CWT, 23

DC, 11,59

Directed Wavelet Covariance, 35, 90-92

estimation, 36

Partial, 47, 48, 55

properties of the Estimators, 37

DWC, 35

dwc, 90-92

DWT, 23

Evolutionary Spectrum, 15, 17

filters

high-pass, 21

low-pass, 21

Fourier Transform, 5

FT, 5

Function

Heavisine, 27

Linear Filter, 47

Locally Stationary Processes, 15, 18

Mallat's algorithm, 25

Model

Vector Autoregressive, 38, 39, 42

Vector Moving Average, 43

MODWT, 28

MRA, 22, 23, 25

Parseval Relations, 6

Partial Coherence, 11

Partial Directed Coherence, 11, 89
Partial Directed Wavelet Covariance, 47, 48, 55, 93-98

PDC, $11,13,89$

pDWC, 47

pdwc, 93-95

ppDWC, 48, 55

ppdwc, 96-98

SDF, 6

Spectral Analysis, 5

Spectrum

Cross, 8

Evolutionary, 15

Partial Cross, 11

Spectral Density Function, 6

Stationary Processes

Strict, 6

Weakly, 7

tv-VAR(p), 48

VAR, 38, 39, 42

time varying, 48

VMA, 43

Wavelet, 19

coefficients, 21

Coherence, 33

Covariance, 32

father, 21

Haar, 20

Mexican hat, 20

Morlet, 20

mother, 20

scaling coefficients, 21

Wavelet Coherence, 89

WTC, 33, 89 